\title{
The role of neurohumoral modulation in fracture healing
}

Citation for published version (APA):

Hofman, M. (2020). The role of neurohumoral modulation in fracture healing: lifting a tip of the veil.

[Doctoral Thesis, Maastricht University]. Maastricht University. https://doi.org/10.26481/dis.20201202mh

Document status and date:

Published: 01/01/2020

DOI:

10.26481/dis.20201202mh

Document Version:

Publisher's PDF, also known as Version of record

\section{Please check the document version of this publication:}

- A submitted manuscript is the version of the article upon submission and before peer-review. There can be important differences between the submitted version and the official published version of record.

People interested in the research are advised to contact the author for the final version of the publication, or visit the DOI to the publisher's website.

- The final author version and the galley proof are versions of the publication after peer review.

- The final published version features the final layout of the paper including the volume, issue and page numbers.

Link to publication

\footnotetext{
General rights rights.

- You may freely distribute the URL identifying the publication in the public portal. please follow below link for the End User Agreement:

www.umlib.nl/taverne-license

Take down policy

If you believe that this document breaches copyright please contact us at:

repository@maastrichtuniversity.nl

providing details and we will investigate your claim.
}

Copyright and moral rights for the publications made accessible in the public portal are retained by the authors and/or other copyright owners and it is a condition of accessing publications that users recognise and abide by the legal requirements associated with these

- Users may download and print one copy of any publication from the public portal for the purpose of private study or research.

- You may not further distribute the material or use it for any profit-making activity or commercial gain

If the publication is distributed under the terms of Article $25 \mathrm{fa}$ of the Dutch Copyright Act, indicated by the "Taverne" license above, 



\section{The role of neurohumoral modulation in fracture healing \\ - lifting a tip of the veil -}


ISBN

Author

Title

Cover illustration

About the cover

Design / Lay-out

Printing
978-94-6423-009-3

Hofman, Martijn

The role of neurohumoral modulation in fracture healing - lifting a tip of the veil -

This image of the sculpture `Unifix` made by Caius Burri for the 'Kunstpfad der Universität Ulm' in 1987, originally photographed by Andreas F. Borchert in 2019 was adapted by Martijn Hofman [licensed under Creative Commons CCBY-SA 4.0 International license].

Caius Burri about his sculpture 'Unifix:

"As a biological substrate, the oblique discontinuous oak tree stem symbols a pseudarthrotic fracture; the metal parts correspond stylistically and aesthetically to the so called external fixator, which is applied in patients throughout the world for open fractures, infections, or lengthening of extremities."

\section{Martijn Hofman}

ProefschriftMaken || www.proefschriftmaken.nl

() Copyright by Martijn Hofman, 2020

All rights reserved. No part of this publication, except for the figures published under Creative Commons CC-licensing, may be reproduced, stored in a retrieval system or transmitted, in any form or by any means, electronic, mechanical, photocopying, recording or otherwise, without prior permission of the author or the copyright-owning journals for previous published chapters. 


\title{
The role of neurohumoral modulation in fracture healing - lifting a tip of the veil -
}

\author{
DISSERTATION \\ to obtain the degree of Doctor at the Maastricht University, \\ on the authority of the Rector Magnificus, \\ Prof. dr. Rianne M. Letschert \\ in accordance with the decision of the Board of Deans, \\ to be defended in public \\ on Wednesday, $2^{\text {nd }}$ December 2020, at 10:00 hours \\ by

\section{Martijn Hofman} \\ born on $30^{\text {th }}$ September 1976 , Sittard, the Netherlands
}




\section{SUPERVISORS}

Prof. dr. M. Poeze

Prof. Dr. med. F. Hildebrand

ASSESSMENT COMMITTEE

Prof. dr. L.W. van Rhijn

Prof. dr. M. van Griensven

Prof. dr. L.P.H. Leenen

dr. H.M.J. Janzing

dr. J.P.A.M. Verbruggen
(University Medical Center RWTH Aachen, Germany)

\section{(Chairman)}

(University Medical Center Utrecht, the Netherlands)

(VieCuri Medical Center, Venlo, the Netherlands) 


\section{Contents}

LIST OF RELEVANT TERMS AND ABBREVIATIONS 9

CHAPTER 1: GENERAL INTRODUCTION, AIMS AND OUTLINE OF THIS THESIS 15 1.1. FRACTURE HEALING 16

1.2. DISTURBED FRACTURE HEALING 19

1.3. THE ROLE OF ANGIOGENESIS IN FRACTURE HEALING 25

1.4. THE ROLE OF BIOLOGICAL FACTORS IN BONE HEALING

1.5. AIMS AND OUTLINE OF THE PRESENT THESIS

1.6. REFERENCES

CHAPTER 2: IMPROVED FRACTURE HEALING IN PATIENTS WITH CONCOMITANT TRAUMATIC BRAIN INJURY: PROVEN OR NOT?

2.1. INTRODUCTION $\quad 75$

2.2. METHODS $\quad 75$

2.3. FRACTURE HEALING

2.4. TRAUMATIC BRAIN INJURY $\quad 77$

2.5. REVIEW OF THE LITERATURE

2.6. DISCUSSION $\quad 85$

2.7. CONCLUSIONS $\quad 88$

2.8. ACKNOWLEDGEMENTS $\quad 89$

2.9. REFERENCES $\quad 89$

CHAPTER 3: RISK FACTORS OF NON-UNION IN INTRAMEDULLARY STABILIZED DIAPHYSEAL LONG BONE FRACTURES: IDENTIFYING THE ROLE OF FRACTURE STABILIZATION STRATEGIES AND CONCOMITANT INJURIES. 95

3.1. INTRODUCTION 97

3.2. MATERIALS AND METHODS 97

$\begin{array}{ll}\text { 3.3. RESULTS } & 100\end{array}$

$\begin{array}{ll}\text { 3.4. DISCUSSION } & 101\end{array}$

$\begin{array}{ll}\text { 3.5. CONCLUSION } & 104\end{array}$

3.6. ACKNOWLEDGEMENTS 104

3.7. REFERENCES 104

CHAPTER 4: EFFECT OF NEUROKININ-1-RECEPTOR BLOCKAGE ON FRACTURE $\begin{array}{lr}\text { HEALING IN RATS. } & 107\end{array}$

4.1. INTRODUCTION 109

4.2. METHODS 110

4.3. RESULTS 115

4.4. DISCUSSION 117

4.5. ACKNOWLEDGEMENTS $\quad 120$

4.6. REFERENCES 121 
CHAPTER 5: GAIT ANALYSIS AND MUSCLE WEIGHT ANALYSIS AFTER LOWER EXTREMITY FRACTURES IN A SMALL ANIMAL MODEL.

5.1. INTRODUCTION

5.2. METHODS

5.3. RESULTS

5.4. DISCUSSION

5.5. CONCLUSIONS

5.6. ACKNOWLEDGEMENTS

CHAPTER 6: ARGININE AVAILABILITY IN REAMED INTRAMEDULLARY ASPIRATE AS PREDICTOR OF OUTCOME IN NON-UNION HEALING, A PILOT STUDY.

6.2. PATIENTS \& METHODS

6.3. RESULTS

6.4. DISCUSSION

CHAPTER 7: THE IMPACT OF PLASMA-DERIVED MICRO-VESICLES FROM A FEMORAL FRACTURE ANIMAL MODEL ON OSTEOBLAST FUNCTION.

7.1. INTRODUCTION

7.2. MATERIALS AND METHODS

7.3. RESULTS

7.4. DISCUSSION

7.5. CONCLUSIONS

7.6. ACKNOWLEDGEMENTS

7.7. REFERENCES

CHAPTER 8: ALTERED CELL SURFACE RECEPTOR DYNAMICS AND CIRCULATORY OCCURRENCE OF NEUTROPHILS IN A SMALL ANIMAL FRACTURE MODEL. 169

8.1. INTRODUCTION

8.2. MATERIALS AND METHODS

8.3. RESULTS

8.4. DISCUSSION

8.5. CONCLUSIONS

8.6. ACKNOWLEDGEMENTS

CHAPTER 9: THE IMPACT OF INTRAMEDULLARY NAILING ON THE CHARACTERISTICS OF THE PULMONARY NEUTROPHIL POOL IN RODENTS. 189

9.1. INTRODUCTION

9.2. MATERIALS AND METHODS

9.3. RESULTS

9.4. DISCUSSION

9.5. CONCLUSION

9.6. REFERENCES 
CHAPTER 10: GENERAL DISCUSSION \& CONCLUSIONS OF THIS THESIS 10.1. PART I: INFLUENCE OF TBI ON FRACTURE HEALING

10.2. PART II: NEURO-HUMORAL MODULATION OF FRACTURE HEALING

10.3. CONCLUSIONS OF THIS THESIS

10.4. REFERENCES

Chapter 11: Summary

11.1. PART I: INFLUENCE OF TBI ON FRACTURE HEALING

11.2. PART II: NEURO-HUMORAL MODULATION OF FRACTURE HEALING

APPENDIX IA: SUMMARY IN DUTCH

APPENDIX IB: SUMMARY IN GERMAN

APPENDIX II: VALORISATION

APPENDIX III: ACKNOWLEDGEMENTS

APPENDIX IV: CURRICULUM VITAE 

List of relevant terms and abbreviations 
arachidonic acid

ABG

ACTH

autologous bone graft

$\mathrm{ADH}$

adrenocorticotropin hormone

AIS

antidiuretic hormone

abbreviated injury scale

ALI

acute lung injury

ALP

alkaline phosphatase

ANOVA analysis of variance

AO

APC

Arbeitsgemeinschaft für Osteosynthesefragen

APC-Cy7

AQP

ARDS

ARG

Arg1

AS

ASA

ASL

ASS

ATLS

AU

AVP

allophycocyanin/Cy7 ${ }^{\circledR}$

aquaporin

acute respiratory distress syndrome

arginine

Arginase 1

argininosuccinate

American college of anaesthesiologists

arginine-succinate lyase

arginine-succinate synthetase

advanced trauma life support

arbitrary units

arginine vasopressin

B BALF

BBB

BGLAP

BMC

broncho-alveolar lavage fluid

blood brain barrier

BMD

BMI

BMP

BMSC

bone gamma carboxylglutamate protein / osteocalcin

bone mineral content

bone mineral density

body mass index

bone morphogenetic protein

bone marrow stromal cell

C CARS

CATK

CCI

counter anti-inflammatory response syndrome

cathepsin $\mathrm{K}$

Charlson comorbidity index

$\mathrm{Ccl}$

CC chemokine-ligand

Ccr

CC chemokine-receptor

CD11a (LFA-1) lymphocyte function associated antigen-1

CD11b (Mac-1) macrophage-1 antigen

CD49d (VLA-4) very late antigen-4 / $\alpha_{4}$-integrin

CD62L L-selectin

cDNA complementary deoxyribonucleic acid

CGRP calcitonin gene-related peptide

CI

CIT

CM

CNS

COMB-4

COX

CPP

CRBP-1

$\mathrm{CRH}$

CSF

$\mathrm{CXCl}$

confidence interval

citrulline

culture medium

central nervous system

$\mathrm{CXCr}$

a mixture of citrulline, paullinia cupana, ginger, and muira puama

cyclooxygenase

cerebral perfusion pressure

cellular retinol-binding protein 1

corticotropin releasing hormone

cerebrospinal fluid

CXC chemokine-ligand

CXC chemokine-receptor

D DALYS

DAMP

DAPI

disability-adjusted life-years

DCO

damage associated molecular pattern

4',6-diamidino-2-phenylindole

DCS

damage control orthopaedics

damage control surgery 


\begin{tabular}{|c|c|c|}
\hline & $\begin{array}{l}\text { DMEM } \\
\text { DNA } \\
\text { DPO }\end{array}$ & $\begin{array}{l}\text { Dulbecco's modified eagle medium } \\
\text { deoxyribonucleic acid } \\
\text { days post-operative }\end{array}$ \\
\hline $\mathbf{E}$ & $\begin{array}{l}\text { ECM } \\
\text { EDTA } \\
\text { ELISA } \\
\text { EM } \\
\text { eNOS / NOS-3 } \\
\text { ETC }\end{array}$ & $\begin{array}{l}\text { extracellular matrix } \\
\text { ethylenediaminetetraacetic acid } \\
\text { enzyme-linked immunosorbent assay } \\
\text { electronic microscopy } \\
\text { endothelial nitric oxide synthase } \\
\text { early total care }\end{array}$ \\
\hline $\mathbf{F}$ & $\begin{array}{l}\text { FACS } \\
\text { FBS } \\
\text { FELASA } \\
\text { FES } \\
\text { FGF } \\
\text { FITC } \\
\text { FSH }\end{array}$ & $\begin{array}{l}\text { fluorescence-activated cell sorting } \\
\text { foetal bovine serum } \\
\text { federation of European laboratory animal science } \\
\text { associations } \\
\text { fat embolism syndrome } \\
\text { fibroblast growth factor } \\
\text { fluorescein isothiocyanate } \\
\text { follicle stimulating hormone }\end{array}$ \\
\hline G & $\begin{array}{l}\text { GAM } \\
\text { GCS } \\
\text { GDF } \\
\beta \text {-GP } \\
\text { GV-SOLAS }\end{array}$ & $\begin{array}{l}\text { gene activated matrices } \\
\text { Glasgow coma scale } \\
\text { growth differentiation factor } \\
\beta \text {-Glycerol phosphate } \\
\text { German society of laboratory animal science }\end{array}$ \\
\hline $\mathbf{H}$ & $\begin{array}{l}\text { HALE } \\
\text { Hif- } 1 \alpha \\
\text { HMGB1 } \\
\text { HO } \\
\text { HPA system }\end{array}$ & $\begin{array}{l}\text { health adjusted life expectancy } \\
\text { hypoxia-inducible factor- } 1 \alpha \text { protein } \\
\text { high-mobility group box } 1 \\
\text { heterotopic ossifications } \\
\text { hypothalamic-pituitary-adrenal system }\end{array}$ \\
\hline I & $\begin{array}{l}\text { ICP } \\
\text { IFN- } \gamma \\
\text { IGF } \\
\text { IL } \\
\text { IMN } \\
\text { iNOS (NOS-2) } \\
\text { IQR } \\
\text { ISS } \\
\text { IZKF }\end{array}$ & $\begin{array}{l}\text { intracranial pressure } \\
\text { interferon gamma } \\
\text { insulin-like growth factor } \\
\text { interleukin } \\
\text { intramedullary nailing } \\
\text { inducible nitric oxide synthase } \\
\text { interquartile range } \\
\text { injury severity score } \\
\text { interdisciplinary center for clinical research }\end{array}$ \\
\hline $\mathbf{K}$ & KKS & kallikrein-kinin system \\
\hline $\mathbf{L}$ & $\begin{array}{l}\text { LFA-1 } \\
\text { L-selectin }\end{array}$ & $\begin{array}{l}\text { state agency for nature, environment and consumer } \\
\text { Protection } \\
\text { lymphocyte function-associated antigen } 1 \text { (integrin on } \\
\text { neutrophils consisting of CD11a) } \\
\text { CD62L (selectin on neutrophils) }\end{array}$ \\
\hline M & 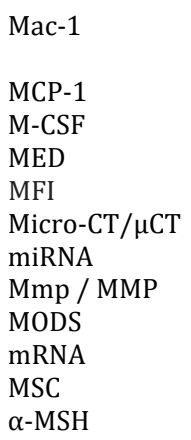 & $\begin{array}{l}\text { macrophage- } 1 \text { antigen / integrin } \alpha_{\mathrm{M}} \beta_{2} \text { (integrin on } \\
\text { neutrophils consisting of CD11b and CD18) } \\
\text { monocyte chemoattractant protein } 1 \\
\text { macrophage colony-stimulating factor } \\
\text { median } \\
\text { mean fluorescence intensity } \\
\text { micro-computed tomography } \\
\text { micro ribonucleic acid } \\
\text { matrix-metalloproteases } \\
\text { multiple organ dysfunction syndrome } \\
\text { messenger ribonucleic acid } \\
\text { mesenchymal stem / stromal cell } \\
\alpha \text {-melanocyte stimulating hormone }\end{array}$ \\
\hline
\end{tabular}


3-(4,5-Dimethylthiazol-2-yl)-2,5-diphenyltetrazolium bromide

MV micro-vesicle

MVCM micro-vesicles containing culture medium

nAChR

NET

NETosis

$N F-\kappa B$

NGF

NK1-R

NK cell

nNOS/NOS-1

NO

NOS

NSAID

NUSS

$\mathbf{0}$

OP-1/BMP-7

OPG

OR

ORN

OSF

$\mathbf{P}$

PAMP

PAI-1

PAR-1

PBS

PCM

PCR

PDGF

PE-Cy5.5

PG

$\mathrm{PGE}_{2}$

$\mathrm{PGI}_{2}$

PMN

POMC

PPIA

PRP

PRR

PTH

Q $\quad$ qPCR

qRT-PCR

R

RAAS

RANKL

RBC

RIA

RP-1

RPE

RUNX

S SD rat

SDF-1

SDS

SE

SEM

SIRS

SMAD nicotinic acetylcholine receptor

neutrophil extracellular trap

the release of neutrophil extracellular traps (NETs) resulting in neutrophil death through a different pathway than apoptosis or necrosis

nuclear factor kappa-light-chain-enhancer of activated B

cells

nerve growth factor

neurokinin-1-tachykinin-receptor

Natural killer cell

neuronal nitric oxide synthase

nitric oxide

nitric oxide synthethase

non-steroidal anti-inflammatory drugs

non-union scoring system

osteogenic protein-1

osteoprotegerin

odds ratio

ornithine

osteoblast stimulating factor

pathogen associated molecular pattern

plasminogen activator inhibitor-1

proteinase-activated receptor 1

phosphate buffered saline

plasma contained culture medium

polymerase chain reaction

platelet-derived growth factor

phosphoethanolamine-cyanine 5.5

prostaglandin

prostaglandin $\mathrm{E}_{2}$

prostacyclin

polymorph nuclear leukocytes

prohormone pro-opiomelanocortin

peptidylprolyl isomerase A

platelet rich plasma

pattern recognition receptors

parathyroid hormone

quantitative polymerase chain reaction

quantitative real-time polymerase chain reaction

renin-angiotensin-aldosterone system

receptor activator of nuclear factor kappa-B ligand

red blood cell

reamer-irrigator-aspirator

monoclonal antibody against rat neutrophils

R-phycoerythrin

runt-related transcription factor

Sprague-Dawley rat

stromal cell-derived factor-1

safe definitive surgery

standard error

standard error of the mean

systemic inflammatory response syndrome

signalling mothers against decapentaplegic 
single nucleotide polymorphism

serine protease 7

osterix protein

statistical package for the social sciences

side scatter

T TBI

TCC

$\mathrm{TF}$

TG

TGF

TNF- $\alpha$

TNFR

tPA

TRAP

TWIST

$\mathrm{TXA}_{2}$

traumatic brain injury

terminal complement complex

tissue factor

transglutaminase

transforming growth factor

tumour necrosis factor- $\alpha$

tumour necrosis factor receptor

tissue plasminogen activator

tartrate-resistant acid phosphatase

twist-related protein

thromboxane A2

V VEGF

VLA-4

W WGA

Wnt

vascular endothelial growth factor

very late antigen- 4 / Integrin $\alpha 4 \beta 1$ (integrin ion neutrophils consisting of CD $49 \mathrm{~d}$ and CD29)

wheat germ agglutinin

wingless/Integrated 
General introduction, aims and outline of this thesis

Martijn Hofman 


\subsection{FRACTURE HEALING}

\subsubsection{Epidemiology, social and economic burdens of fractures}

Bone fractures are common musculoskeletal injuries 1. Approximately 6.8 million fractures occur annually in the United States ${ }^{2}$, and an epidemiological study from 2008 revealed that the incidence rate of fractures in England was 3.6 cases per hundred people per year ${ }^{3}$. In addition, the study showed that the lifetime prevalence of fractures was $>50 \%$ in males aged 34-55 years and approximately $40 \%$ in women over 75 years old. Furthermore, it has been observed that the fracture location is associated with age. In this context, a study indicated that approximately one-third of hip, vertebral and other fractures (pelvis, clavicle, humerus, wrist, forearm and leg) occurred in people aged 50-64 years ${ }^{4}$, whereas tibial fractures more frequently occurred in adults who are less than or close to 50 years old 5 .

The social and economic burdens of fractures are considerable ${ }^{6}$, and as the patient population continues to age worldwide, the incidence of osteoporosis-related fractures is rising ${ }^{7}$. Researchers have reported that expenditures on osteoporosis-related fractures in the United States range from $\$ 10$ billion to $\$ 17$ billion every year, and it is estimated that those annual costs could be $\$ 25.3$ billion by $2025^{8}$. Ström et al. found that osteoporosis-related costs in 2011 were approximately $€ 30.7$ billion in six European countries, including Germany, France, the United Kingdom, Italy, Spain and Sweden. It is important to note that the majority of osteoporosis-related medical costs in those six countries were attributed to fractures, while only $4.7 \%$ of total costs were related to pharmacological treatment 9 .

It is obvious that both direct medical healthcare costs and indirect expenditures due to disability or loss of work productivity are strongly correlated with the healing time, which varies according to fracture location, classification and treatment method 10 . According to Parziale et al., the healing time ranged from 6-8 weeks for proximal humerus fractures to 12-16 weeks for femoral fractures. An additional period of 12-52 weeks and 15-30 weeks might be added to reach a complete recovery for proximal humerus fractures and femoral fractures, respectively. Approximately one-third of patients with a unilateral fracture of the lower extremity were unable to return to work within one year after initial injury ${ }^{10}$. In the last three decades, the rate of fracture healing has been greatly improved because of an increased understanding of fracture repair and advancements in fixation instruments and techniques.

According to the Global Burden of Disease Study 2017 11, life expectancy increased worldwide by 7.4 years, from 65.6 years (65.3-65.8) in 1990 to 73.0 years (72.7-73.3) in 2017. However, in most countries, the increase in the number of years of life expected to be lived in good health was smaller than the increase in overall life expectancy, indicating more years lived in poor health. Those findings challenge medical science to improve Health Adjusted Life Expectancy (HALE) by further improving diagnostics and treatments for conditions which have a high burden of disease. At the same time, this sharpens the current discussion regarding whether the increasing possibilities of medical diagnostics and therapies outweigh the associated increasing costs to society. The burden of disease is expressed in disability-adjusted life-years (DALYs), and traumatological causes play a considerable role in the overall burden of disease. Among the top 30 leading causes of DALYs, road injuries (men: 49.8 million [95\% CI 47.3-52.1] DALYs; women: 18.0 million [16.6-19.4] DALYs) and falls (men: 21.0 million [17.6- 
24.9] DALYs; women: 15.0 million [12.3-18.0] DALYs) have had stable positions over the last 30 years for both men and women ${ }^{11}$.

\subsubsection{The process of fracture healing}

Contrary to the healing of other tissues, bone tissue in fracture healing regenerates to the original tissue with the same biochemical and biomechanical characteristics as before the injury. That is one reason why fracture healing takes longer than the healing of soft tissues ${ }^{12}$.

The healing of bone is a complex, dynamic process which entails different stages. Adequate fracture healing depends on the interaction of many biomechanical and biological factors, as described in the 'diamond concept' of fracture healing by Giannoudis and colleagues ${ }^{13}$. According to this concept, Giannoudis explains that for adequate bone restoration and regeneration to occur, besides growth factors, scaffolds and mesenchymal stem cells (triangular concept), the mechanical environment plays at least an equally important role. In the fairly complex process of bone repair, the coordination of cells and numerous mediators is required ${ }^{14}$. Primary (direct) and secondary (indirect) bone healing are considered to be the two basic histological routes of fracture repair ${ }^{13}$. Primary fracture healing is the goal every surgeon wants to achieve because the healing period is shorter than for indirect fracture repair ${ }^{15}$. However, this type of bone fracture healing is rare because it requires certain necessary conditions, including a rigid fixation and an anatomic reposition of the fracture fragments without a bone gap between both sides of the fracture ends. In fact, most fractures, including those treated operatively, heal via secondary healing, including intramembranous and endochondral ossification. Generally, the secondary fracture healing process is differentiated into four partially overlapping stages: the inflammation stage, the soft (or bridging) callus formation stage, the hard (or medullary) callus formation stage and the remodelling stage (Figure 1.1.).

\subsubsection{Inflammation stage}

The inflammatory response starts with the fracture and the immediate excretion of inflammatory exudate into the fracture gap. This phase is necessary to initiate the healing process, and it peaks within the first 24 hours and disappears completely about 5-7 days after fracture. In this stage the fracture haematoma originates, organises and forms a link between the fracture fragments. The fracture haematoma consists of coagulated bleeding around the fracture sides and provides a template for callus formation ${ }^{16}$. The platelets in the haematoma are aggregated and degranulated. They then release growth factors and stimulate the subsequent chemotactic signals. Different cells, including monocytes, lymphocytes and macrophages, are attracted to the haematoma, and cytokines including TNF- $\alpha$ (tumour necrosis factor- $\alpha$ ) and interleukins (ILs) such as interleukin-1 (IL-1), IL-6, IL-11 and IL-18 are secreted and enhanced within the first few days following the initial fracture ${ }^{17}$. Other inflammatory cells are attracted by the pro-inflammatory cytokines mentioned above, leading to the further aggregation of platelets and the stimulation of angiogenesis. Hypoxic changes are present at both sides of the fracture site and in bone marrow during the first few days because the blood supply to the affected bone is disrupted ${ }^{18}$. Macrophages participate in regulating the early stage of fracture repair as they not only phagocytize and kill necrotic tissue but also regulate revascularisation ${ }^{19}$. 

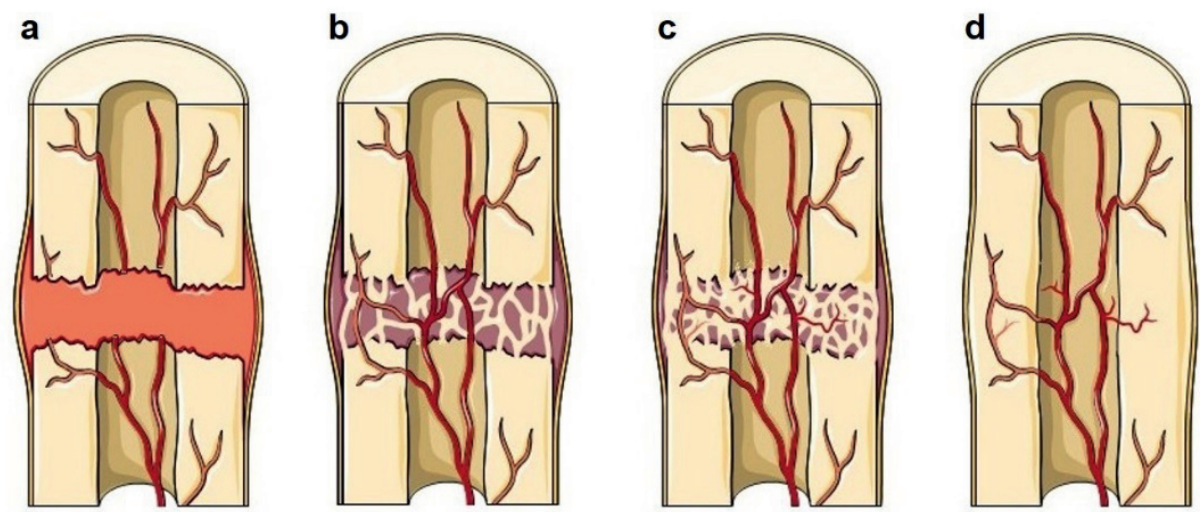

Figure 1.1. A schematic representation of the secondary fracture healing process. (a) Inflammation stage: the haematoma is formed as a result of a disruption of blood vessels after trauma, then numerous inflammatory cells and biological factors occur to initiate the bone healing process. (b) Soft callus formation stage: neovascularization occurs (angiogenesis) and fibroblasts deposit (fibro) cartilage tissue. (c) Hard callus formation stage: the immature woven bone is replaced by woven bone through intramembranous or endochondral bone formation. (d) Remodelling stage: the woven bone is replaced by lamellar bone and the biomechanical properties are restored. [This illustration by Smart Servier Medical Art is licensed under Creative Commons CC-BY-SA 3.0 (https://smart.servier.com/)].

The recruitment, proliferation and differentiation of mesenchymal stem cells (MSCs) are also necessary for bone regeneration. First, MSCs are recruited to the fracture site by stromal cell-derived factor-1 (SDF-1) and G-protein-coupled receptor CXCr-4 20,21. Then, TNF- $\alpha$ stimulates the differentiation of MSCs into osteoblasts and chondrocytes by activating the expression of tumour necrosis factor receptor-1 (TNFR-1) and tumour necrosis factor receptor-2 (TNFR-2), which are expressed on osteoblasts and osteoclasts ${ }^{22}$. IL- 1 and IL- 6 are two essential interleukins for bone repair because either the generation of cartilaginous callus or angiogenesis is mediated by IL-1 23,24 , while the differentiation of osteoblasts and osteoclasts is regulated by IL- $6{ }^{25}$. The whole process of the inflammatory phase promotes the generation of granulation tissue and the formation of new blood vessels.

\subsubsection{Soft (or bridging) callus formation stage}

By the end of the first week following trauma, a cartilaginous callus tissue called a 'softtissue callus' or 'pro-callus' is generated surrounding the fracture site. This soft tissue callus consists of different components, including fibrous connective tissue, newly generated blood vessels, collagen fibres, cartilage and immature woven bone ${ }^{26,27}$. The fibroblasts within the granulation haematoma, influenced by different growth factors, such as transforming growth factor- $\beta 1$ (TGF- $\beta 1$ ) and platelet-derived growth factor (PDGF), deposit fibrocartilage and cartilage tissue, which forms a weak bridge between the fracture fragments and reinforces the stability of the fracture site ${ }^{28-30}$. This stage of fracture healing lasts for approximately $2-3$ weeks. 


\subsubsection{Hard (or medullary) callus formation stage}

The cartilaginous callus will gradually turn into cartilage because chondrocytes are formed under hypoxic conditions in the peripheral callus regulated by cytokines such as PDGF, insulin-like growth factor 1 (IGF-1) and bone morphogenetic proteins (BMPs), including BMP-2, BMP-5 and BMP-6 ${ }^{31}$. Numerous molecular signals, including transforming growth factor- $\beta 2$ (TGF- $\beta 2$ ), transforming growth factor- $\beta 3$ (TGF- $\beta 3$ ) and BMPs, further promote the calcification process. In order to increase the stability of the fracture site, the immature woven bone is eventually replaced by woven bone through intramembranous or endochondral bone formation ${ }^{17,26,32}$. During the process, which can take between 6 and 12 weeks depending on the fracture type and the anatomical fracture site, the organic matrix (predominantly consisting of collagen) is mineralised by the deposition of hydroxyapatite. Due to continuous proliferation, chondrocytes become hypertrophic, and the appearance of the mineralised extracellular matrix represents the beginning of the process of endochondral ossification ${ }^{33}$.

\subsubsection{Remodelling stage}

The remodelling of bone is an imperative quality of bone tissue, taking place after fractures but also as a lifelong process in normal healthy bone. With regard to fracture healing, remodelling is the last stage in bone healing, starting immediately after woven bone has formed but perhaps lasting for several years until completion ${ }^{30}$. The objective of this phase is to achieve the same mechanical strength as normal bones have by replacing the woven bone with lamellar bone ${ }^{16}$. The substitution of (woven) bone takes place according to Wolff's law, which describes the response of bone transformation to mechanical loading and the possibility of altering its mechanical properties according to this loading stress ${ }^{34}$. Eventually, when the applied mechanical loading is sufficient, bone will be restored to its original form and strength, involving bone resorption by osteoclasts and the formation of new bone by osteoblasts. This osteoclastic and osteoblastic activity is generated via a polarisation of the involved long bone by axial loading of the crystalline bone matrix ${ }^{35}$. In this polarisation process, one electropositive convex side activating osteoclasts emerges and one electronegative concave side activating osteoblasts emerges ${ }^{32,35}$. After an erosive pit is generated by osteoclasts resorbing the woven bone on the calcified surface, also known as 'Howship's lacuna', osteoblasts deposit the new bone in the eroded pit ${ }^{36}$. The remodelling phase is complete after a central medullary cavity has formed in the internal callus and the lamellar bone has substituted the external callus 35 .

The success of the remodelling stage depends to a great extent on a sufficient blood supply and the stability of the fracture site ${ }^{37}$. Fractures can be stabilised in two ways. Absolute stability can be achieved by anatomic reduction and interfragmentary compression, without micro-motion of the fracture parts, which results in primary bone healing; relative stability occurs when the fracture parts are reduced in a functional way and controlled micro-motion is still possible between the fragments, which results in secondary bone healing.

\subsection{DISTURBED FRACTURE HEALING}

Although the understanding of fracture pathology and the treatment of fractures has greatly evolved in recent decades, deranged fracture healing in the sense of delayed unions and non-unions is still very difficult to predict at the time of injury and during 
the course of the healing process ${ }^{38}$. Considering the number of studies published on the topic of non-unions, in our opinion, the clinical and socioeconomic importance of nonunions is probably underestimated. Most papers on non-unions report a general incidence of non-union of approximately 5-10\%, but almost all of those papers based their figures on the information from one book by Praemer and colleagues on musculoskeletal epidemiology ${ }^{39}$. On the other hand, in 2017, Mills published a paper in which she demonstrated overall non-union rates of $1.9 \%$ (men: $1.5 \%$; women: $2.3 \%$ ) in a large patient population ${ }^{40}$. For specific fractures in particular age groups, however, the risk increased to $9 \%$. The non-union incidence peaked in men aged 25-34 and in women aged 65-74, and the overall non-union risk peaked in early adulthood and decreased with rising age ${ }^{40,41}$. Mills and Simpson also evaluated non-union incidence for children and found a very low risk, probably due to the relatively strong periosteum, the excellent vascularity and the great healing potential of paediatric bone. The nonunion risk for girls in general and boys aged under 14 years was $\leq 0.2 \%$. For older teenage boys (15-19 years), the incidence raised to approximately $0.5 \%{ }^{41}$.

Independent of the exact incidence of non-union, the direct and indirect costs of nonunions are very high and they are projected to increase over the next 10-20 years as the rate of trauma-related morbidity in the young adult population rises ${ }^{42}$, the survival rate for patients with severe injuries improves ${ }^{43-46}$ and the age of the overall patient population increases.

Non-unions consume increasing health care resources and double the median costs of care compared with normally healing fractures 47,48 . Furthermore, non-unions and especially non-unions of the lower extremity are a significant clinical problem with a tremendous impact on the quality of life of the patient ${ }^{48-50}$. Various studies on quality of life show an enormous incapacitating effect on the physical and mental health of such patients 50,51 . Non-union patients need more opioids and suffer more from pain and depression because their physical health is comparable with patients with end-stage hip arthrosis and worse than those with congestive heart failure 47,49 . That is why patients with non-unions on average have longer hospital stays, outpatient treatment and rehabilitation periods 51 .

The direct costs to hospital organisations for individual non-union therapy range from $£ 7000$ to $£ 79,000$ per person ${ }^{41,52}$. Indirect costs of non-unions are difficult to determine as they differ according to each patient's personal life and occupational circumstances. For instance, only $60 \%$ of non-union patients return to work within the first year after trauma. In addition, they show a significant decrease in productivity ${ }^{47,48}$. Hak et al. estimated the indirect costs to be $67 \%-79 \%$ of the total costs of a tibia fracture in the Canadian health care system and $82.8 \%-93.3 \%$ of all costs for tibia fracture patients in European health care systems. ${ }^{53}$ Furthermore, the costs can even accumulate through litigation procedure costs, which are increasing, particularly when there is associated residual deformity ${ }^{38}$.

Another difficulty in comparing different studies concerning non-unions is the diversity of non-union definitions used in the literature. Despite that diversity, in our opinion, exact time frames to define non-union are not the most important measure because different bones have different healing times. More important in defining non-union is the fact that the healing of the particular bone does not occur in the expected time, that there is no progression in the healing process and that a successful union is not expected without intervention ${ }^{54,55}$. Among all the bones in the human body, long bones such as the femur and the tibia need the longest time to heal. and those bones also have 
among the highest non-union rates $\left(13.9 \%\right.$ and $14 \%$, respectively) ${ }^{56}$. In general, physicians should realise that almost all fractures should heal within 3 to 4 months, ${ }^{55}$ and when healing has not occurred within this time range, one should become suspicious. Furthermore, the suggested waiting period of 9 months to diagnose the nonunion before intervention may be unrealistic in an economic climate in which healthcare and social systems are under more and more strain ${ }^{57}$. Patient education and expectations must be addressed in conjunction with advancements in techniques and surgeons' competencies to reduce the social and financial burden.

Besides the definition in terms of time, non-unions can be classified according to Weber and Cech, who categorised non-unions into hypertrophic and atrophic ${ }^{58}$. Hypertrophic or hyper-vascular non-unions have an adequate vascularity and biological activity to progress to union, but the healing is limited by bony instability. Conversely, atrophic or avascular non-unions lack vascularity and biological healing potential and show no evidence of healing. The classic view on this, as Calori et al. described, was that atrophic non-unions were associated with factors acting directly on the early phases of fracture healing while hypertrophic non-unions relate mostly to factors acting on the 'reorganisation' phase of bone healing ${ }^{59}$. The general opinion now is more nuanced and not as clear-cut. Avascular non-unions can be further subgrouped by the fracture pattern - torsion wedge, comminuted, defect and atrophic - and Weber and Cech further subdivided non-unions by radiological appearance (elephant foot, horse hoof and oligotrophic) ${ }^{58}$. Moreover, it is important to consider the possibility of an infection in all cases of delayed union or non-union and particularly in high-energy open fractures.

All conditions which impair fracture healing can lead to non-union and are divers from origin $13,40,60$. Some conditions arise from injury characteristics, such as mono vs. polytraumatisation, fracture localisation (metaphyseal vs. diaphyseal), fracture type (open vs. closed, simple vs. comminuted, percent cortical contact), bone quality (juvenile vs. osteoporotic), or vascularity of bone, periosteum and soft-tissue condition (impaired blood supply, excessive periosteal stripping and fragment denudation, presence of a compartment syndrome, need for a flap). Other conditions originate from patient characteristics, such as sex, age, general health (malnutrition, adipositas, osteoarthritis with rheumatoid arthritis, vitamin D deficiency, hepatic and renal disorders), activity level, metabolic status (anaemia, diabetes mellitus, hormone deficiencies), use of drugs (anti-inflammatory medication, anti-depressant medication, steroids, anticoagulants, bisphosphonates, opioids, anticonvulsants, benzodiazepines, insulin, antibiotics, diuretics), alcohol and tobacco. Some conditions ensue from the interventions performed, such as fracture treatment (conservative vs. operative, reamed vs. unreamed), instability of fixation, axis deviation, and infection 47,56,61-65. As the personal and socio-economic consequences of non-unions are so serious, all influenceable factors which affect normal bone healing have to be considered as possible research targets to improve the treatment of predisposed patients with acute fractures or (nonpredisposed) patients with delayed or non-unions.

As Giannoudis et al. ${ }^{13}$ described in their diamond concept that for adequate fracture healing, in addition to biological factors, such as growth factors, scaffolds and mesenchymal stem cells, the biomechanical factors are of equal importance, those factors should be considered for prophylaxis and treatment of non-unions. First of all, excellent biological conditions should be established at the fracture site. In acute fractures, that means that the fracture haematoma should not be removed with the 
reduction because it contains a significant number of growth factors, cytokines, osteogenic and angiogenic factors. Moreover, the disposure of the fracture ends should be as limited as possible to retain as much periosteum as possible. Then, the proper biomechanical conditions should be created, either with a rigid fixation and compression on the fracture fragments or with a biologic osteosynthesis in cases of comminuted fractures. In cases of non-union, the following treatment algorithm has been established through the years. A debridement and removal of infectious and/or necrotic tissue should be performed, a transplantation of autologous bone graft (ABG), containing mesenchymal stem cells, with osteoinductive, osteoconductive and osteogenic properties is carried out and, if required, a re-osteosynthesis with implant replacement and restoration of mechanical stability is accomplished ${ }^{66}$.

\subsubsection{Poly-trauma and fracture healing}

In poly-traumatised patients, the pathophysiologic reaction is first predominated by a swift development of hypovolemia reaching a shock state in a short period of time. Subsequently, the organism attempts to minimise the consequences of the injuries by activating different homeostatic mechanisms to save vital organ functions. Ultimately, the organism aims to return to the pre-traumatic situation ${ }^{67}$.

Although for several years the prevailing opinion on this pathophysiologic reaction was that it consisted of three physiological phases (i.e., a hypodynamic flow phase, a hyperdynamic flow phase and a recovery phase) ${ }^{68}$, it is now known that the reaction is more complex and consists of multiple pathways which constitute a fragile equilibrium between inflammatory (systemic inflammatory response syndrome [SIRS]) and antiinflammatory (counter anti-inflammatory response syndrome [CARS]) mechanisms ${ }^{69}$. The inflammatory response of the body caused by major trauma starts with an immunoinflammatory reaction in which different cells are activated, which damage the endothelial membrane in the vital organs of the body. Those cells, including monocytes, lymphocytes, natural killer cells and polymorphonuclear leukocytes (PMN), are then able to spread to other organs, injure those organs and fully evolve into SIRS and even multiple organ dysfunction syndrome (MODS) ${ }^{70,71}$. In cases of major physiologic stress, including polytrauma, the dysregulation of the neutrophil response as part of the PMN pool is known to be crucial in the origin of SIRS as well as MODS 72,73. Besides cytokines, such as Interleukin-1, $-6,-8$ and -10 and TNF, which are known to play an important communicative role in the activation of immune cells and in the origin of SIRS and MODS, there is also another group of signalling molecules, which are scrutinised in various studies for the part they play in such phenomena ${ }^{67}$. Those alarmins or endogenous danger cells, as they are called, mainly stimulate the cells (i.e., T cells, neutrophils, monocytes, macrophages, natural killer cells and dendritic cells) of the natural immune response in the early phases, and they are released at the site of injury by necrotic or apoptotic cells 74,75 . High-mobility group box 1 (HMGB1) is the most important representative of this group besides antibacterial peptides, S100 and heatshock proteins ${ }^{76}$. The expanse of this SIRS is basically determined by the extensiveness of the initial traumatic impact ('first hit'), but it can be increased by additional treatment interventions ('second hit') 77,78. An excessive SIRS reaction can lead to complications, such as acute respiratory distress syndrome (ARDS), MODS and death. Various studies have demonstrated that the inflammatory response in poly-traumatised patients with a combination of injuries is magnified compared with the response of the individual injuries and thereby affects the outcome ${ }^{79}$. 
On the other hand, if the CARS, which develops at the same time, is super-dominant, a suppression of the immune system follows, which is related to infectious and septic conditions of the patient.

This improved understanding of the immune-mediated systemic response of the body to poly-trauma has led to an important change in the initial treatment of such patients. In the pre-hospital setting, the 'scoop and run' concept has replaced the 'stay and play' concept in the vast majority of cases.

In the emergency department, treatment is directed towards preventing the occurrence of the 'triad of death', consisting of coagulopathy, acidosis and hypothermia. The triad is mainly caused by hypovolemia, hypoxia and heat loss. Therefore, the treatment during the 'golden hour of shock' seeks to control blood and heat loss, stabilise oxygen saturation and restore coagulation.

For the subsequent treatment options, the physiological condition of the patient leads, and the condition can be subdivided into four categories: stable, borderline, unstable and extremis ${ }^{67}$. For the initial surgical treatment of the patients, except for the stable patients, a shift from early total care (ETC) to damage control surgery (DCS) has taken place.

Importantly, traumatic brain injury often represents a major threat for the outcome and survival of trauma patients and alters the approach from ETC to DCS in order to reduce the secondary hit to the brain. The neuroendocrine axis is activated after trauma by pain, fear, by-products of metabolism which cross the blood-brain barrier, and primary brain damage itself ${ }^{67}$. Subsequently, the hypothalamus and the sympathetic-adrenal system together with signals from the renin-angiotensin system increase heart rate and vasoconstriction in an attempt to control blood pressure.

In such poly-traumatised patients, the healing of fractures is often challenging and nonunions are an established phenomenon ${ }^{51,80}$. Hildebrand and colleagues endorse the fact that interactions between local and systemic inflammatory reactions play an important role in fracture healing in patients with multiple injuries, but they also emphasise the influence of concomitant injuries, such as chest trauma, severe tissue injuries and haemorrhagic shock, on bone metabolism and fracture healing ${ }^{64}$.

\subsubsection{Neuro-trauma and fracture healing}

The overall prognosis of patients with (concomitant) traumatic brain injury depends on both the primary and secondary brain damage. At the time of the initial traumatic impact on neuro-cranium and brain tissue, the primary brain injury originates and consists of concussion, contusion, shear injuries, lacerations and axonal stretching ${ }^{81}$. In cases of severe primary brain injury, in which lesions of neurons, axons and microglia cells occur, the mortality rate is very high. Subsequent to the primary injury, a delayed complex immunological, biochemical and physiological pathomechanism, which continues for several days to weeks, results in secondary brain damage ${ }^{81,82}$. That secondary brain damage is a multifactorial process which is caused and influenced by different processes, such as excitotoxicity, inflammation, oedema, cell death, mitochondrial damage, magnesium depletion, the production of free radicals and damage to the blood brain barrier 82,83 .

The complex pathomechanism which leads to secondary brain damage also influences other healing processes in the body such as bone regeneration. According to the clinical findings of some experts in the field, patients with concomitant severe TBI often show faster and excessive fracture healing. The alleged relationship between TBI and fracture 
healing has been the subject of clinical and experimental research during the last three decades. Despite the long history of studies, in which evidence of a positive correlation between TBI and osteogenesis is rising ${ }^{84-96}$. there is no conclusive evidence for the relationship and there are many clinical and experimental studies taking a stand for or against this supposed relationship ${ }^{97,98}$. Moreover, the pathophysiologic mechanisms underlying the supposed osteogenic phenomena in patients with TBI are multifactorial and far from clarified ${ }^{99,100}$. In the literature, various possible mechanisms of action have been postulated without unambiguous proof of a specific pathophysiologic mechanism. The following possible working principles have been discussed in recent years.

TBI leads to a complex metabolic, inflammatory and neuroendocrine reaction, and various authors have assumed that the osteoinductive potential of the serum increases as a result. Furthermore, it has been discussed that based on the changes of neuronal activity in the injured brain tissue, an increased synthesis of osteoinductive factors occurs, which are then transported to the fracture site. Within this frame, important significance, without definitive proof, has been attributed to different cytokines, proteins, peptides, cells and neurotransmitters (i.e., substance P and calcitonin generelated peptide [CGRP]) ${ }^{84-89,93}$.

In recent years, several authors have described a neurogenic inflammatory mechanism, which could influence callus formation and the healing of fractures, via different neuropeptides, which are released after both TBI and bone fractures ${ }^{101,102}$. Although the exact pathophysiologic mechanism underlying the interaction is not known, Substance $\mathrm{P}$ and CGRP are two neuropeptides which are upregulated after TBI and released after peripheral nerve injury (by fractures), and they are supposed to be involved in the healing of fractures.

Supportive of this theory are the findings of different authors which demonstrate a positive influence of such neuropeptides, released by peripheral sensory nerve endings in the bone tissue, on the quality of bone regeneration and the integrity of bone 103,104. It is known that bone is innervated via sensory nerves in the periosteum and nerves accompanying the nutrient vessels of the Haversian canals in the bone. However, although until now 'classic' synapses between nerves and bone cells are not proven, Jones et al. were able to show that there are nerve fibres with an active expression of different neurogenic ligands, which are in close contact with bone cells ${ }^{103}$. In case of a fracture, those nerve endings are injured, regenerate and grow back into the callus tissue. That proliferation of nerves elapses simultaneously with the formation of callus tissue and the subsequent bone remodelling, implying the active involvement of peripheral nerves in these processes ${ }^{104}$. Corresponding to those findings, Santavirta et al. demonstrated a lack of peripheral innervation of bone in non-unions ${ }^{105}$.

Moreover, several studies have demonstrated by utilising either combined motoric, sensory and autonomic denervation ${ }^{106,107}$ or complete sensory nerve-ending blockage 108,109 that complete peripheral nerve transection $106,107,110$ and the selective blockade of sensory nerve endings 108,109 can impair fracture healing.

That negative influence of a blockage of efferent sensory nerves on the quality of callus formation and fracture healing is due to the inability to release Substance P and CGRP 108,109 . Further studies have shown that the nociceptive system of sensory nerves is essential for the initialisation of the inflammatory process in fracture and wound healing ${ }^{104}$, that the concentration of sensory neuropeptides are upregulated in the first 24 hours after fracture ${ }^{111}$ and that the number of nerve fibres increases in cases of bony defects 106,112. According to those results, it is plausible that neuropeptides, and 
especially substance $\mathrm{P}$ and CGRP, are of significant importance for the healing of fractures.

One neuropeptide of particular relevance to bone healing seems to be Substance P, which shows simultaneous effects in neurogenic and osteogenic activities. Substance $P$ seems to have a significant involvement in bone metabolism, formation and resorption as well as in the osteogenic activity of bone marrow stromal cells and osteogenic cell lines 113-116. However, to date, the specific role of single neuropeptides in bone healing has not been established 97,98 .

In another concept, which finds support in the literature, the bone morphogenetic proteins (BMPs) as members of the transforming growth factor $\beta$ (TGF- $\beta$ ) superfamily play a role in increased bone healing in patients with concomitant TBI. The BMPs transfer their signals via binding to the transmembrane receptor complex, which incorporates serine-threonine kinase activity ${ }^{117}$. The complex is activated after neurogenic trauma, and the subsequent activation of the signalling mothers against decapentaplegic (SMAD) gene family modifies the cellular transcription activity ${ }^{118-120 .}$ Three members of the BMP family (BMP-2, BMP-4 and OP-1 [formerly called BMP-7]) have the opportunity to stimulate bone growth in peripheral locations 121,122. Furthermore, it is supposed that after bone fractures as well as after TBI, a cascade in which MSCs play an important role is initiated. BMPs released from the injured bone matrix support that initiation ${ }^{123}$. As multipotent cells, the MSCs can differentiate into various mesenchymal cell lines, which enables the development of different tissues, such as bone, cartilage, fat, etc 124,125. Besides proteins produced by the MSCs themselves, other mediators, which are not further specified in studies, released by injured brain tissue are described to stimulate MSCs and osteoprogenitor cells ${ }^{84}$.

\subsubsection{Heterotopic ossification (HO)}

It is in this context that several authors claim that the increased callus formation in patients with concomitant TBI is due to the generation of heterotopic ossifications. Heterotopic ossification as pathological bone formation in extra-skeletal tissue can have a traumatic, neurogenic or genetic genesis. Although the exact pathophysiology of heterotopic ossification is not yet known, the idea is that the formation of such ossifications shows a similarity to the normal fracture healing process of osteogenesis, osteoinduction and osteoconduction. Nauth et al. suggests that osteoprogenitor cells, which have a pathologic differentiation in response to dysregulated BMP signalling, play a role in the formation of heterotopic ossifications(HOs) ${ }^{126}$. The combination of an inflammatory response, with increased levels of IL-6 and monocyte chemoattractant protein 1 (MCP-1), and dysregulated BMP signalling would mainly be responsible for the osteoinduction in $\mathrm{HO}$ formation ${ }^{126}$. Injured soft tissue forms an ideal environment for the development of such ossifications. In contrast to the involvement of the abovementioned inflammation response factors in orthopaedic and blast injury patients with $\mathrm{HO}$, the knowledge of the pathogenesis of $\mathrm{HO}$ in patients with concomitant TBI is still exiguous. The involvement of e.g. catecholamines, leptin, CGRP and substance P, has been hypothesised 126,127 .

\subsection{THE ROLE OF ANGIOGENESIS IN FRACTURE HEALING}

In recent decades, several studies proved that the vascular status is of major importance for normal bone homeostasis and restoration of bony defects. The mechanism of fracture healing is a multifactorial process in which many different cytokines, 
hormones, proteins, etc., play a role. Above all, virtually all of those studies emphasise the key role of revascularization and angiogenesis as an essential prerequisite for new bone formation 128-132. Vasculogenesis and angiogenesis are two basic processes of blood vessel formation ${ }^{133}$. (Figure 1.2.) Unlike vasculogenesis, in which the vascular network is initially formed from angioblastic stem cells, the development of a new blood vessel from a pre-existing vascularity is defined as angiogenesis 133-135. Revascularization is important for the physiology of bone formation, bone growth and remodelling 136,137 because it transports the nutrients, oxygen, hormones, cytokines and numerous cells which are required by bone tissues $31,130,138$. Those complex processes of neovascularization and angiogenesis are regulated by different pro- and antiangiogenic mediators, such as vascular endothelial growth factor (VEGF), hypoxiainducible factor- $1 \alpha$ protein (Hif- $1 \alpha$ ), fibroblast growth factor (FGF), TGF, IGF, interleukins, BMPs and NO 129,132,139.

\section{Hemangioblast}

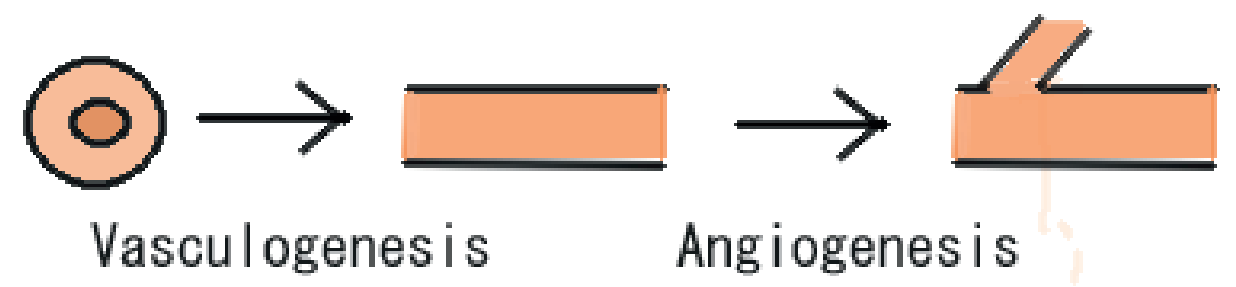

Figure 1.2. The relationship between vasculogenesis and angiogenesis. Endothelial progenitors first differentiate into clusters of endothelial cells, and the primary vascular system is formed by the fusion of endothelial cells (vasculogenesis). The primary vascular system is remodelled to form a mature vascular system through angiogenesis. [This illustration by puttinpurin1108 is licensed under Creative Commons CC-PD 1.0 (commons.wikimedia.org)].

The reduction of bone mass and bone formation is correlated with insufficient blood supply ${ }^{132,140}$. Furthermore, along with the deepening research, surgeons are realising that a successful fracture repair critically depends on successful angiogenesis ${ }^{141-143}$.

When fractures occur, the periosteum and blood vessels between the fractured ends are damaged, subsequently changing the local microenvironment. One study has shown that the overall blood flow of the bone after a fracture or osteotomy is reduced immediately ${ }^{144}$. The circulation of cortical bone can be reduced by approximately $50 \%$ due to the physiological contraction of the periosteal and intramedullary vessels following trauma, while the partial pressure of oxygen in the centre of the damaged tissues can even be decreased to $0 \%-2 \%{ }^{145}$. The hypoxic environment around the fracture site can induce the release of various cytokines, which promote the differentiation of stromal precursor cells in the periosteum, bone marrow and surrounding soft tissue towards osteoblasts and chondrocytes.

The process of intramembranous bone formation occurs in the adjacent ischemic area and requires not only the anaerobic metabolism of osteoblasts but also the transportation of nutrients and mineral through new blood vessels ${ }^{146}$. Chondrocytes are located at the centre of the hypoxic region where the complete absence of blood 
perfusion mostly occurs. The proliferation, differentiation and matrix deposition of those cells is associated with angiogenesis in order to achieve successful cartilage ossification 147. Moreover, it has been reported that angiogenesis occurs prior to osteogenesis, and the latter is a vascular-dependent process 148,149. In case of compromised angiogenesis or microcirculation at the fracture site, $50 \%$ of such fractures will result in a pseudarthrosis or non-union ${ }^{128}$. Moreover, in vivo research with rat models has shown that delayed fractures or non-unions occur if angiogenesis is inhibited ${ }^{143}$. This could possibly be explained by the decisive role of angiogenesis in every single step of fracture healing, but the vascular network also disposes imperative growth factors, such as cytokines, hormones, proteins, amino acids, etc., to the fracture site and its surrounding tissue. The endothelium functions as a communication network for that surrounding tissue ${ }^{130}$.

\subsection{THE ROLE OF BIOLOGICAL FACTORS IN BONE HEALING}

Besides the musculoskeletal system, other biological systems such as the immune system play an extremely important role in bone regeneration. In the complex process of bone regeneration and fracture healing growth factors, cytokines and chemokines play a major role in addition to the cellular involvement of immune cells, osteocytes and osteoclasts $28,33,150,151$. The inflammation stage at the beginning of fracture healing is considered to be a very important phase because that local, non-systemic inflammation response has to provide an excellent environment for fracture healing to evolve. That is achieved by clearing necrotic debris and forming good granulation tissue ${ }^{152}$. The coordinated progress of that inflammation response has to be assured by a delicate equilibrium between different inflammatory and anti-inflammatory cytokines and other biological factors because uncontrolled inflammation can impede the fracture healing process ${ }^{153}$.

\subsubsection{Genetic factors}

As with almost all other diseases, different genetic factors seem to play a role in bone regeneration and fracture healing.

For instance, the regulation of gene expression associated with numerous biological processes is performed by micro-ribonucleic acids (miRNAs), and there is growing evidence that miRNAs are also important for bone formation, resorption and remodelling and the development of non-unions ${ }^{154}$. Waki and colleagues showed that there is an upregulation of different miRNAs in adequate healing tissues. In another in vivo experiment, they showed that another set of miRNAs are upregulated in cases of non-unions 155,156 .

Furthermore, it is suggested that single nucleotide polymorphisms (SNPs), which are variations in a single nucleotide that occur at a specific position in the genome, where each variation is present to some degree within a population, are responsible for the progression of a fracture into a non-union 157. Those SNPs are found in four genes encoding for the BMP pathway (BMP-2, BMP-7, Noggin, Smad6) 157,158. Supportive of this theory are the findings of different in vivo and ex vivo studies that the distribution of BMP genes, amongst others, can increase bone healing 159-161. Ali and colleagues suggested the involvement of a single T/G genotype at SNP rs3753793 in the CYR61 gene, encoding for a widespread signalling molecule, in the development of non-unions 162 . 
Based on a comparison of the findings with those for patients with normal fracture healing, a platelet-derived growth factor (PDGF) haplotype was reported to be associated with aseptic non-union ${ }^{163}$.

For the expression of different genes in the fracture healing process, it is of utmost importance that the expression is well balanced because an excessive expression could damage the structure and function of related cells, leading to impaired bone healing. That was demonstrated by Zimmermann and colleagues, who showed an increased expression of different genes in non-unions, which normally coordinate the production and stabilisation of the extracellular matrix, the induction of cell differentiation and proliferation and the cytoskeleton ${ }^{164}$.

The correction or adaptation of some of these genetic changes and the modulation of bone metabolism in patients with disturbed fracture healing are possible indications for gene therapy.

Gene therapy for bone repair can be divided into (stem) cell-mediated therapy and acellular therapy. The cell-mediated strategy, in which a cellular component is used as the carrying vector, is very complex and difficult to implement in the clinical situation, because the tissue has to be selected, transduced in vitro and then re-implanted into the bony defect, mostly in a second operation 165,166 . However, for instance, Xing et al. showed some promising results of bone marrow mesenchymal stem cells (BMSCs) transfected with IGF-1 gene promoting fracture healing in an in vivo study ${ }^{167}$.

Acellular direct gene therapy comprises of different techniques using viruses (vectors), plasmids (extrachromosomal DNA molecule), and gene activated matrices (GAM) impregnated with plasmids to transfer therapeutic cDNA (complementary DNA) into the host cell ${ }^{165}$. These methods have been performed in vivo directly administered as a suspension into the bony defect or freeze-dried to an allograft scaffold 165 . Main advantages of genes over recombinant osteogenic proteins are a sustained secretion of therapeutic proteins by transfected cells, a more physiological manner in which the therapeutic proteins are presented, due to the posttranslational processing in the host cell, the ability of repeated administration, and the low cost of gene production 166,168. Concerning the efficacy of transferring genetic information to cells, viral vectors are superior to plasmids ${ }^{169}$. Vectors from adenoviruses or adeno-associated viruses are used to transduce mesenchymal stem cells and chondrocytes and different bone morphogenetic proteins (i.e. BMP-2, BMP-4, BMP-7) are used in these vector types to enhance osteogenesis ${ }^{170-172}$. Although gene therapy is still at an early stage, it has a tremendous potential of correcting genetic mutations or improving bone metabolism by delivering osteogenic and angiogenic proteins to the fracture site. Therefore, it might become the most valuable therapy option for fracture patients with a genetic predisposition towards delayed union and non-union development.

\subsubsection{Plasma proteins}

The systemic and local inflammatory responses after trauma are coordinated by three cascade systems, which are activated by pro-inflammatory mediators, toxins and/or direct tissue damage. Those interrelated systems of plasma proteins are the clotting system, the complement system, and the kallikrein-kinin system ${ }^{173}$. 


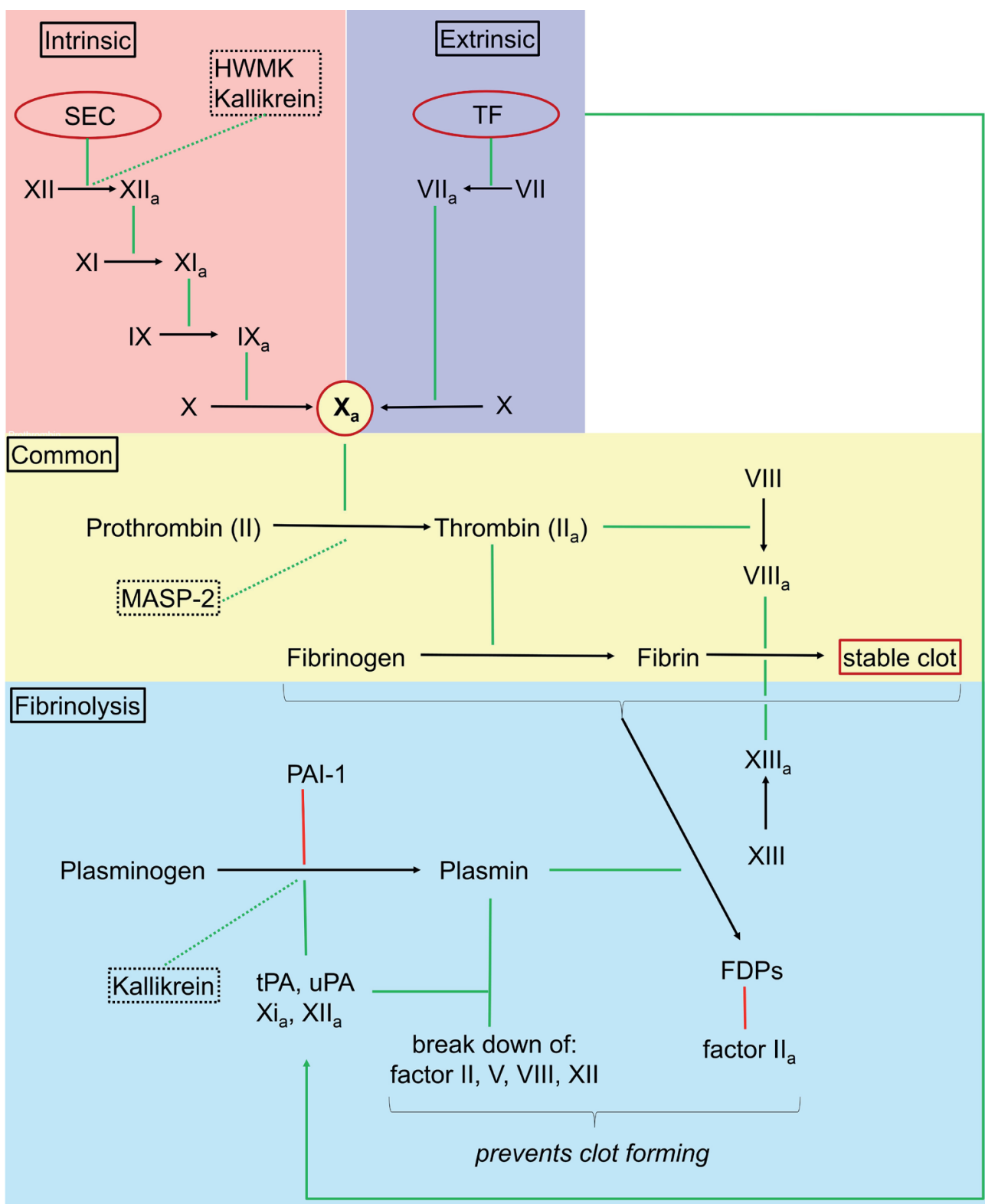

Figure 1.3. A schematic representation of the intrinsic, extrinsic and common clotting cascade and the fibrinolysis pathway. FDPs: fibrin degradation products, HMWK: high molecular weight kininogen, MASP-2: Mannan-binding lectin serine protease 2, PAI-1: plasminogen activator inhibitor-1, SEC: subendothelial collagen, TF: tissue factor, tPA: tissue plasminogen activator, uPA: urokinase plasminogen activator. [This illustration was created by Martijn Hofman and is licensed under Creative Commons CC-BY-SA 4.0] 


\subsubsection{Clotting system}

Bones are vascularized extensively and in case of a fracture this vascularity is disrupted. The clotting system (Figure 1.3.) tries to limit the blood loss by forming an organised haematoma, that provides the connection between the different bone fragments 174 . Furthermore, different components of the clotting system influence the inflammatory response to injury and interact with other systems like the Kallekrein-kinin-system and the complement system ${ }^{74}$.

Thrombin. Thrombin (coagulation factor IIa) seems to have an anabolic function in bone by stimulating the proliferation of different osteoblasts and their functions ${ }^{175}$. In the study of Sato et al., they suggest that thrombin is another cytokine regulating osteoblast function and fracture healing. They demonstrated that thrombin stimulated osteoblasts to express proteinase-activated receptor 1 (PAR1 / coagulation factor II receptor), to produce monocyte chemoattractant protein-1 (MCP-1), tissue factor (TF), macrophage colony stimulating factor (MCSF) and Il-6, and to reduce the expression of plasminogen activator inhibitor-1 (PAI-1).

The production of MCP-1 and MCSF leads to the recruitment of monocytes and macrophages into the fracture haematoma and the expression of PAR1 and of TF leads to the subsequent production of more thrombin via the extrinsic coagulation pathway. Both phenomena are important for the maintenance of inflammation in the early stages of fracture healing ${ }^{176}$. Vi et al. also showed that the presence of macrophages in the fracture healing process is vital for callus formation and the quality of fracture healing 177. Further, thrombin seems to have an important role in the balance between coagulation, through increasing the expression of TF by osteoblasts and fibrinolysis, through reducing the expression of PAI-1 by osteoblasts. It further causes a burst of prostaglandin E2 (PGE2) and prostacyclin (PGI2) production by osteoblasts via the B2 receptor ${ }^{175}$.

Fibrin. In the process of fracture healing, fibrin, which is formed by both the intrinsic and extrinsic coagulation pathway, has a bimodal function. First of all, fibrin is essential for the formation of a stable blood clot surrounding the fracture site. The fibrin matrix retains platelets and forms a reservoir for platelet associated growth factors and vasoactive molecules. It further promotes the recruitment of inflammatory and mesenchymal progenitor cells into the fracture haematoma through specific integrin / receptor interactions. Above that, it provides the structural mesh like framework for the initial phase of tissue repair. In the in vivo study of Vasconcelos et al. it was demonstrated that a fibrinogen-scaffold (fibrinogen is the precursor of fibrin) favours a pro-regenerative local environment, followed by changes in the systemic immune cell balance and advanced fracture repair ${ }^{178}$. Fibrinogen further improves the angiogenesis through the binding of VEGF, an important neovascularization factor, with high affinity ${ }^{179,180 .}$.

On the other hand, excessive fibrin deposition or an inefficient break down of the fibrin clot impairs the vasculogenesis, which occurs from both ends of the fracture site and is essential for new bone to form. For instance, Yuasa et al. demonstrated in plasminogendeficient mice, in which as a result fibrinolysis was disturbed, that fracture vascularisation and bone healing was impaired ${ }^{181}$.

Fibrin stabilizing factor (coagulation factor XIII). Factor XIII is a member of the transglutaminase (TG) family, and it is found in many different cells, such as platelets, 
monocytes, macrophages, chondrocytes, osteoblasts and osteocytes. Factor XIII plays a role in the formation of extracellular matrix, by stabilizing the interaction of microtubules with the cell membrane, through which collagen and fibronectin, as important components of the extracellular matrix, are secreted. Collagen and fibronectin form stable interaction with osteonectin, which binds Calcium and osteopontin, which is a structural linking protein in bone. TG as a catalyst for this interaction plays an essential role in the mineralization and collagen deposition of the extracellular bone matrix ${ }^{182,183}$.

Plasmin. Plasmin degrades fibrin into fibrin degradation products and dissolves the stable fibrin clot. Primarily, this plasmin-mediated fibrinolysis is necessary for the vascularisation of the soft- and hard-callus tissue. The recruitment and direction of essential endothelial and osteoblastic progenitor cells to initiate angio- and osteogenesis is coordinated by hypertrophic chondrocytes by the release of VEGF-A, calcium and phosphate ${ }^{181}$. Plasmin also has a role in the clearance of the fracture site from debris by the recruitment of monocytes and macrophages into the fracture haematoma. Thereby, the inflammatory response in fracture healing is kept within limits independently of the fibrin degradation ${ }^{184}$. Moreover, plasmin supports the release of growth factors, which are important for tissue repair, i.e. TGF- $\beta^{185-187}$ and VEGF 187-189. Schoenecker et al. also demonstrated a direct conducive effect of plasmin on the maturation of osteoblast precursors to mature osteoblasts during fracture healing by stimulation of the mineralization and differentiation of in vitro osteoblast cultures ${ }^{190}$.

Plasminogen, the precursor of plasmin, and its activators urokinase and tissue plasminogen activator (tPA) are released by osteoblasts and osteoclasts, stimulated by cytokines and hormones 191,192. They influence various components of bone remodelling, such as the recruitment of macrophages ${ }^{187}$ and the osteoclastogenesis and the osteoclast / osteoblast interaction ${ }^{193,194}$.

\subsubsection{Complement system}

The proteins of the complement system are numbered $\mathrm{C} 1$ to $\mathrm{C} 9$, and they have different modes of action within the immune system. Normally those plasma proteins circulate inactive in plasma. The activation of this system can be initiated via three different pathways. There are 3 intrinsic pathways (classical, alternative, and Mannose-binding lectin) to activate the complement cascade, with the key-players C3 and C5 triggering the terminal pathway to constitute the Terminal Complement Complex (TCC). Interaction between immune cells, the clotting system, the kallekrein-kinin system and the complement system exist in that different serine proteases, clotting factors as well as serine proteases from macrophages can activate the complement cascade via an extrinsic pathway, independently from the intrinsic pathways ${ }^{195-197}$. (Figure 1.3.) The pore-forming Terminal Complement Complex (TCC) possesses both lytic as well as nonlytic activities. TCC formation on cell membranes can cause lysis of the cell of pathogens and microorganisms. In sub-lytic concentrations the TCC can induce cell signalling pathways, stimulation of pro-inflammatory mediators, mobilisation of immune cells, production of different peptides, free oxygen radicals and cytokines, and modulation of cell cycle and apoptosis ${ }^{198-201}$. Besides the early induction of an inflammatory response to fracture and the elimination of debris and potentially invasive microorganisms at the fracture site, complement factors ( $\mathrm{C} 3 \mathrm{a}$ and $\mathrm{C} 5 \mathrm{a}$ ) also recruit polymorphonuclear 
neutrophils, MSCs, osteoblasts and macrophages to the fracture site ${ }^{202}$. Further, C3 and C5 play an important role in the Cell-to-cell communication between osteoblasts and osteoclasts, which are both very important players in the regulation of the immune response after fracture and of the metabolism of bone ${ }^{202}$. Bergdolt et al. even suggest that via the C5a-receptor on osteoblasts and osteoclasts, C5 upregulates the activity of osteoclasts and the expression of osteoclastic factors, such as RANKL and IL-6 with an enhanced resorption of bone as a consequence ${ }^{203}$.

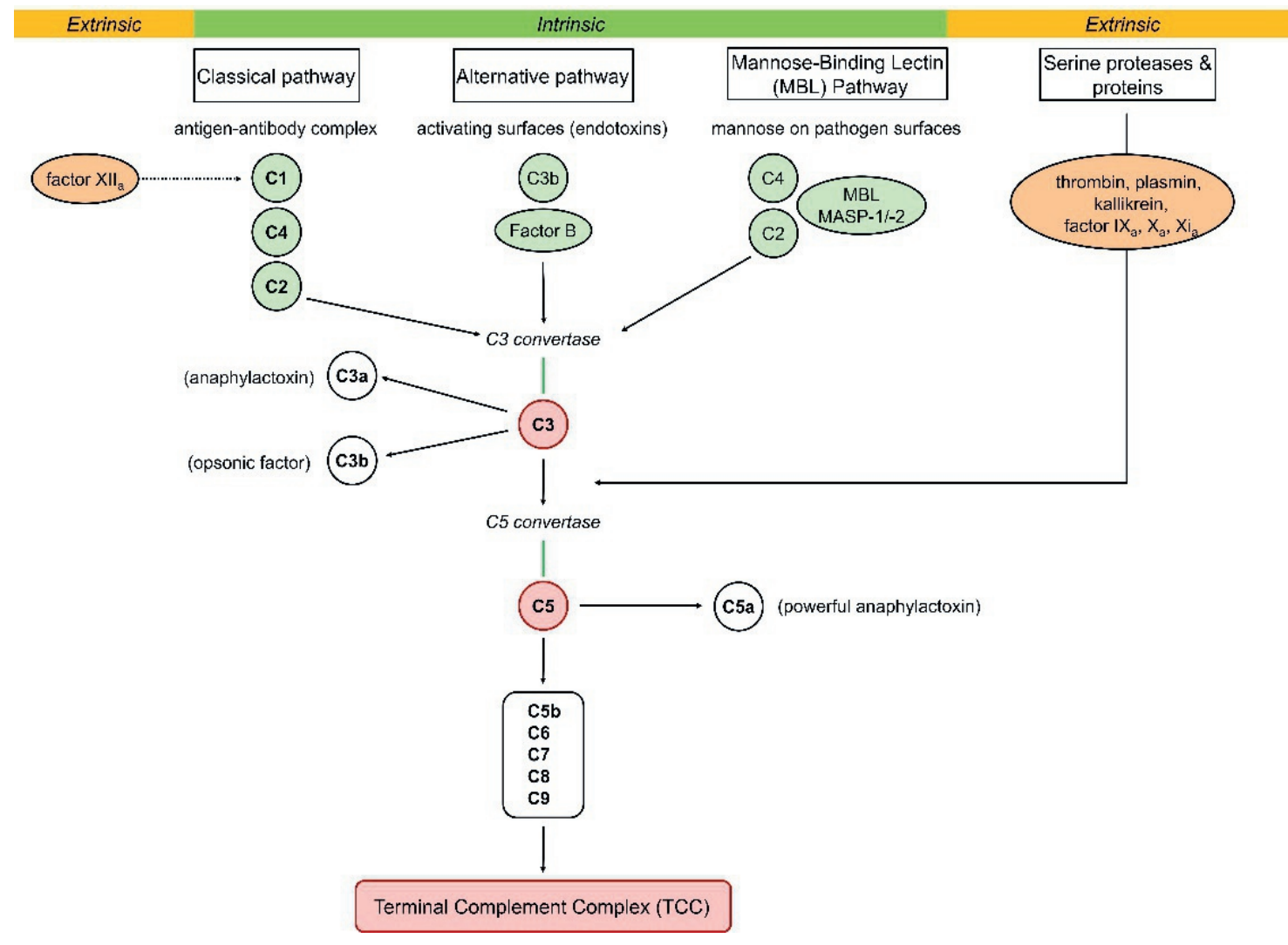

Figure 1.4. A schematic representation of activation pathways of the complement system. There are 3 intrinsic pathways (classical, alternative, and mannose-binding lectin) to activate the complement cascade, with the key-players C3 and C5 triggering the terminal pathway to constitute the Terminal Complement Complex (TCC). There is also an independent extrinsic pathway, involving different serine proteases and proteins from the coagulation system and from immune cells. [This illustration was adapted by Martijn Hofman from 'Figure 1. Complement pathways' by Zaahira Gani published on https://www.immunology.org/publicinformation/bitesized-immunology/systems-and-processes/complement-system (licensed under Creative Commons CC-BY-SA 4.0)]

Supportive to these findings Ehrnthaller et al. showed decreased callus volume and mechanical strength of healing bone in their in vivo study with $\mathrm{C} 3 \%$ and $\mathrm{C} 5 \%$-knockout mice ${ }^{204}$. Mödinger et al. also demonstrated a role for $\mathrm{C} 6$, as an essential component for 
TCC assembly, in the inflammatory response to fracture and in the normal fracture healing process, because in absence of $\mathrm{C} 6$ an increased osteoclast activity was seen with a subsequent impaired bone healing 201. Moreover, Mödinger showed that, under physiological conditions, human bone cells protect themselves against complement attacks by the expression of CD59 glycoprotein, which is able to prevent C 9 from polymerizing and forming the TCC 201.

Besides the activation of the extrinsic complement pathway via several serine proteases and proteins of the coagulation system, the complement and coagulation cascade are strongly interconnected and augment each other via the intrinsic mannose-binding lectin pathway. The binding of MBL to the cell membrane of pathogens causes an activation of MBL-associated serine proteases (MASPs), which subsequently activate proteins from the complement cascade. At fracture sites, MBL also binds to fibrinnetworks and in turn, fibrin stabilises the MBL-MASP complex, enhancing a further activation of complement proteins. MASP-2 (Mannan-binding lectin serine protease 2) is consecutively an enzyme, which can cleave prothrombin into thrombin very potently and thereby augment the fibrin complex ${ }^{205}$. (Figure 1.3. \& 1.4.)

\subsubsection{Kallekrein-kinin system (KKS)}

The most important mediators of the kallikrein-kinin system are bradykinin and kallidin, which are activated in the cascade system via inflammation and tissue damage. (Figure 1.5.) Kallikrein has chemotactic functions, and it also plays a role in the clotting system by stimulating the activation of factor XII and by counterbalancing the clotting cascade by stimulating the conversion of plasminogen to plasmin, activating fibrinolysis 198. Plasma-kallikrein and factor $\mathrm{XII}_{\mathrm{a}}$ are rapidly inactivated by $\mathrm{C1}$-inhibitor from the complement system, antithrombin from the clotting system and $\alpha_{2}$-macroglobulin, mainly synthesized by macrophages. Tissue-kallikrein, however, is not affected by inhibition.

In cases of inflammation in the vicinity of bone, the activity of osteoclasts is induced, which results in bone resorption. This influence on bone metabolism can be elicited by different cytokines, growth factors, and thrombin, but also by the kinins of the KKS. Both kinins act on the B1 and B2 receptor expressed by osteoblasts, which elicit a stimulation of the prostaglandin production. The expression of these receptors can be upregulated by the pro-inflammatory cytokines IL-1 $\beta$ and TNF- $\alpha{ }^{206}$. Prostaglandin increases the formation and activates the differentiation of osteoclast progenitor cells to osteoclasts and these are responsible for subsequent bone resorption 175,206,207. This working mechanism is supported by the study of Zhang et al., in which a decreased expression of the B1 receptor improved local bone metabolism 208.

\subsubsection{Cytokines}

Many studies, preclinical as well as clinical, have proven the value of cytokines in coordinating and modulating bone regeneration and fracture healing in recent years ${ }^{154}$. The four fracture healing stages are all regulated by the expression of cytokines, matrixmetalloproteinases (Mmps), and angiogenic factors. The cytokines can be subdivided into three categories: (1) proinflammatory cytokines, (2) anti-inflammatory cytokines, (3) members of the TGF- $\beta$ superfamily and other growth factors 16 . Cytokines are mainly released by lymphocytes and macrophages but also by epithelial, connective tissue and endothelial cells. The regulatory mechanisms of cytokines can be easily deviated by environmental influencing factors. 


\subsubsection{Pro-inflammatory cytokines}

Proinflammatory cytokines are upregulated within the first hours after trauma and appear mainly at the first stage of fracture healing but are also imputed to influence the remodelling stage of bone regeneration 23,209 . The proinflammatory cytokine group consists of TNF- $\alpha$, IL-1 $\beta$, IL-6, IL-8, IL-12 and IL-17.

Inflammation

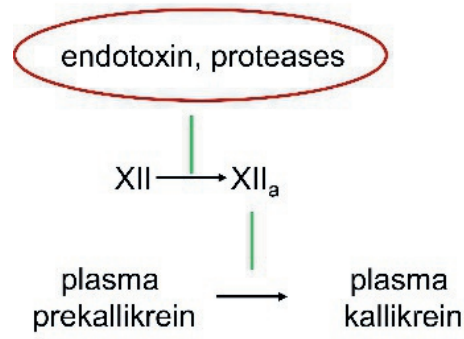

Tissue injury

proteases

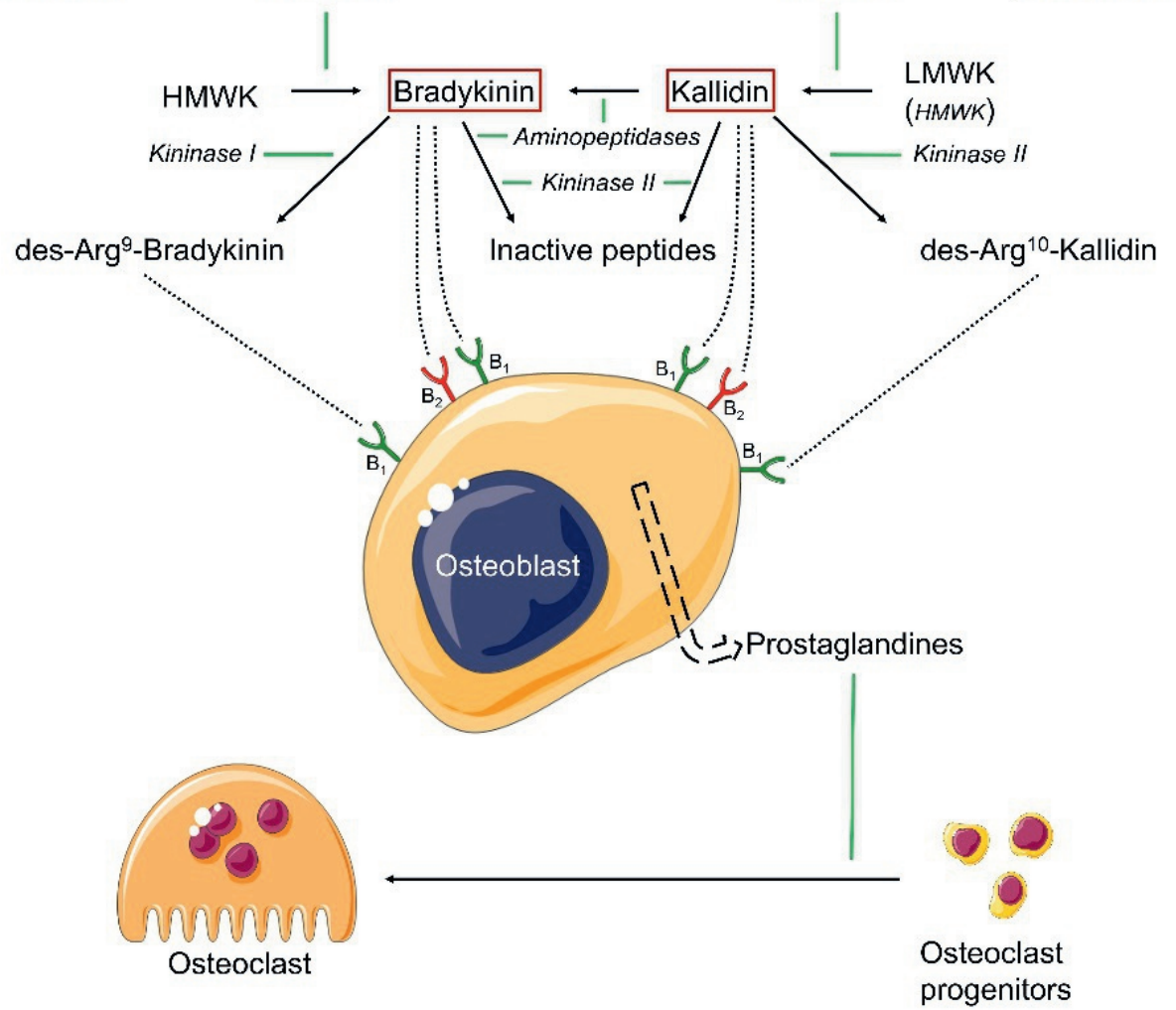

Figure 1.5. A schematic representation of the kallekrein-kinin system in bone metabolism. $B_{1}$ : Gprotein-coupled $\mathrm{B}_{1}$-kinin-receptor, $\mathrm{B}_{2}$ : G-protein-coupled $\mathrm{B}_{2}$-kinin-receptor, HMWK: high molecular weight kininogen, LMWK: low molecular weight kininogen, XII: clotting factor XII or Hageman-Factor, XIIa: activated clotting factor XII,. [This illustration was created by Martijn Hofman (licensed under Creative Commons CC-BYSA 4.0) using images from Smart Servier Medical Art (https://smart.servier.com/)]. 
Tumour necrosis factor alfa (TNF- $\alpha$ ). Together with IL-1 $\beta$, TNF- $\alpha$ is released within the first 2 hours after fracture. Macrophages are the main source of TNF- $\alpha$, which can bind to two receptors, TNFR1 and TNFR2. The effects of TNF- $\alpha$ on bone regeneration are time-sensitive. As one of the first proinflammatory cytokines, the maximum concentration is reached within the first 24 hours after injury and reverts to baseline levels after 72 hours. ${ }^{16}$ During that period, TNF- $\alpha$ increases the endothelial permeability and the responsiveness of mesenchymal cells to chemokines, thereby facilitating the migration of these cells into the fracture site, where they start the osteogenesis 210,211 . Subsequently, it has a positive effect on bone regeneration by upregulation of the innate immune response via support of the production of chemokines, cytokines, growth factors and NO 210,211. Besides those effects, TNF- $\alpha$ also enhances the neutrophil secretion of VEGF and thereby encourages vasculogenesis at the fracture site ${ }^{212}$. Then, apoptosis of chondrocytes and recruitment of osteoclasts is triggered by TNF- $\alpha$, causing soft callus resorption and the induction of endochondral bone formation ${ }^{213,214}$. In the later stages of fracture healing, possibly depending on various expression patterns of TNF- $\alpha$, TNF- $\alpha$ exerts a converse influence on callus mineralisation and fracture healing 215-218. Moreover, the effect of TNF- $\alpha$ on bone regeneration depends on its concentration and is, therefore, dose-dependent. Low-level concentrations of TNF- $\alpha$ lever mesenchymal stem cells migration to the site of injury and their osteogenesis, but at high-level concentrations, TNF- $\alpha$ is counter-productive for bone formation ${ }^{219}$. The negative effect of TNF- $\alpha$ on bone regeneration is also supported by different in vitro studies $215,217,218,220,221$.

Interleukin 1 beta $(I L-1 \beta)$. Interleukin-1 $\beta$ is another cytokine which appears early in the inflammatory stage of fracture healing. Together with TNF- $\alpha, \mathrm{IL}-1 \beta$ is one of the most important inflammatory cytokines in coordinating the local inflammatory response to bone injury ${ }^{28}$. Corresponding to TNF- $\alpha$, IL-1 $\beta$ exerts its effects, which are also timesensitive and dose-dependent. That is, IL-1 $\beta$ first influences bone repair at the inflammatory stage and further on in the process at the soft and hard callus formation stages ${ }^{23}$. IL-1 $\beta$ positively influences osteoclast growth ${ }^{222}$, osteoblast apoptosis and chondrocytic differentiation ${ }^{223}$. Furthermore, it stimulates the upregulation of matrix metalloprotease 13 (MMP-13) activity via the wingless/int1 (Wnt) signalling pathway, which causes the maintenance of bone mass and enhances bone repair ${ }^{224}$. However, the positive effects of IL- $1 \beta$ are only employed in a narrow concentration range of 0.1 $\mathrm{ng} / \mathrm{ml}$ to $0.3 \mathrm{ng} / \mathrm{ml}$; otherwise, IL-1 $\beta$ has the opposite effect ${ }^{153}$.

Interleukin 6 (IL-6). Another important proinflammatory cytokine for bone healing is IL-6, produced by a variety of cells (monocytes, macrophages, neutrophils, T cells, B cells, endothelial cells, smooth muscle cells and fibroblasts) and executing its effect via two signalling pathways. In the IL-6 classic pathway, IL-6 binds to a membraneanchored IL- 6 receptor present on hepatocytes, epithelial cells and leukocytes, and that complex then binds to the receptor subunit gp130, which is a transmembrane glycoprotein, which subsequently initiates the intracellular signalling. ${ }^{225}$ The second pathway is called the IL- 6 trans-signalling pathway, in which the IL- 6 receptor protein is cleft and a soluble receptor (sIL-6R) is created. The receptor binds IL-6 and, after connecting to the receptor subunit gp130, signalling is generated, even in cells which do not express IL-6R 225 .

In poly-traumatised patients, IL- 6 is recognised as an important parameter for determining the severity of an injury, the favourable moment to perform an operation, 
and the prognosis after the polytrauma ${ }^{173}$. IL- 6 is upregulated in two peaks. In the acute inflammatory phase (within the first 24 hours after fracture induction), IL-6 initiates the recruitment and proliferation of MSCs, which are multipotent stromal cells, and their differentiation to the osteogenic cell lines $23,226,227$. Another characteristic of IL- 6 is that it promotes angiogenesis, which is important for the delivery of blood and nutrients to the bone tissue 228,229 .

The second peak occurs at the stage of hard callus formation in which IL- 6 stimulates the endochondral and intramembranous mineralisation of the soft callus ${ }^{230}$. In this stage, IL- 6 advances osteoclastogenesis $25,231,232$, but it also stimulates the overall turnover of healing bone 215,220,228,229.

On the other hand, some studies have found enhanced fracture healing by suppressing the IL- 6 activity in mice ${ }^{233}$, and others state that only the classic IL- 6 signalling pathway and not the IL-6 trans-signalling pathway is crucial for fracture healing ${ }^{234}$.

Interleukin 17 (IL-17). This proinflammatory cytokine is yielded by various cells from the immune system (i.e., B cells, natural killer (NK) cells and mesenchymal cells), but the majority of IL-17 comes from the $\gamma \delta$ T cell, a subset of T cells with a specific T cell receptor expression ${ }^{235}$. The expression of IL-17 can be upregulated by interleukin 12 and interleukin 23 (a member of the IL-12 family) ${ }^{153}$. The IL-17 group mobilises neutrophils and releases inflammatory cytokines and consists of the subtypes IL-17AF 236. Of those subtypes, IL-17A shows inconsistent effects on osteoblastogenesis, depending on the target cells involved 237,238 . Positive effects are exerted on immature mesenchymal cells at the site of injury, but a negative effect is displayed on preosteoblasts $238-241$. Furthermore, Nam and colleagues show that the subtype IL-17F has positive effects on the maturation of osteoblasts ${ }^{242}$.

\subsubsection{Anti-inflammatory cytokines}

Interleukin 4 and 13 (IL-4 and $I L-13)$. Several studies have demonstrated that both interleukin 4 and interleukin 13, as well as the combination of these two cytokines, downregulate bone resorption ${ }^{243,244}$. IL-4 in particular has the capacity to activate the transition of macrophages, from the proinflammatory M1 phenotype to the antiinflammatory M2 phenotype, which enhances osteogenesis and bone regeneration 245.

Interleukin 6 (IL-6). Besides IL-6's proinflammatory function, it also exerts antiinflammatory effects through the generation of anti-inflammatory cytokine and prostaglandin $\mathrm{E}_{2}\left(\mathrm{PGE}_{2}\right)$ release by macrophages. Subsequently, $\mathrm{PGE}_{2}$ coordinates the synthesis of TNF- $\alpha$ and IL-1 $\beta$ and the release of IL-10 173 .

Interleukin $10(I L-10)$. One of the most potent anti-inflammatory cytokines is interleukin 10 (IL-10), which is produced by $\mathrm{T}$ cells or myeloid cells. Through the inhibition of proinflammatory cytokines, such as TNF- $\alpha$, IL-1, IL-6, IL-8 and free oxygen radicals, IL-10 exerts an indirect suppression of bone mass, mechanical strength and bone formation 246,247 .

Interleukin 27 (IL-27). As a member of the interleukin 12 family, IL-27 is secreted by monocytes, macrophages and dendritic cells. The inflammatory response to fracture is substantially antagonised by IL-27. Furthermore, IL-27 has a stimulating effect on the remodelling of bone through the enhancement of osteogenesis, the inhibition of osteoblast apoptosis and diminishing the proinflammatory response 248,249 . 


\subsubsection{Members of the TGF- $\beta$ superfamily and other growth factors}

The TGF- $\beta$ superfamily is a group of related cell regulatory proteins, which can be subdivided into different subfamilies: the TGF- $\beta$ subfamily; the BMPs and growth differentiation factors (GDFs) subfamily; the subfamily of the activins, inhibins and Müllerian-inhibiting substance; and a subfamily of various other important growth factors, including IGFs, PDGF and FGFs. ${ }^{250}$ The most relevant mediators for fracture healing will be discussed below.

Transforming growth factor beta (TGF- $\beta$ ). Transforming growth factor beta has value mainly in the early stages of fracture healing ${ }^{251}$. As a chemotactic modulator of osteogenic differentiation, it stimulates the healing and remodelling of bone via cell division and mobilisation, matrix synthesis and tissue differentiation ${ }^{252-255}$. Interesting data have been demonstrated by Zimmermann and colleagues concerning a possible predictive value of TGF- $\beta$ for delayed fracture healing situations because in normal fracture healing TGF- $\beta$ initially peaks at 2 weeks and then decreases over the subsequent 4 weeks ${ }^{256}$.

Bone morphogenetic proteins (BMPs). There are about 20 different BMPs, but only BMP2 and BMP-7 (or osteogenic protein-1 [OP-1]) are currently used clinically in non-union therapy and as an adjuvant treatment 257,258 . Their effect on bone healing occurs via two pathways. The BMPs trigger the recruitment of MSCs, which then differentiate either to osteoblasts depositing bone material directly or to chondrocytes changing to bone cells along the way ${ }^{259}$. Both BMP-2 and BMP-7 are expressed during the early stages of fracture healing, but BMP-2 exerts its effect throughout the entire fracture healing process. The BMP pathways are susceptible to environmental influences, through which the effect or expression of BMP decreases. For example, nonsteroidal antiinflammatory drugs (NSAIDs), which are widely used painkillers in musculoskeletal injuries, reduce the response of osteoprogenitor cells to BMP and thereby decrease their osteogenic potential ${ }^{260}$. Also, the initial hypoxic state of fracture tissue instigates reduced scavenging, oxidative stress and enhancement of reactive oxygen species (ROS); consequently, BMP-2 expression impairment occurs, leading to insufficient bone healing 261.

In the early stages, BMP-2 is involved in enhancing the inflammatory response, the periosteal activation and the differentiation of stem cells to osteoprogenitor cells. At the later stages, BMP-2 is involved in regulating chondrogenesis and osteogenesis 16,262. BMP-7 exerts its action only after the formation of osteoprogenitor cells in the early stages of fracture healing ${ }^{262}$. According to the literature, BMP-7 has an osteoinductive function 263,264 , but Haubruck et al. were also able to demonstrate an angiogenic effect of BMP-7 in bone regeneration. ${ }^{265}$ Although these two BMPs have been established in the treatment of non-union and are already on the market, the literature is not univocal on their proof of action concerning bone healing. Several studies show positive effects of BMPs on bone regeneration and angiogenesis 13,263,264,266-272. Other studies have been unable to prove any significant advantage of adding BMP-2 or BMP-7 to autogenous bone grafts in non-union treatment, probably due to the initiation of the expression of BMP antagonists after local administration of BMPs 273,274 . There are even studies which mention relevant adverse effects of BMP treatment in non-unions ${ }^{275}$.

Insulin-like growth factors (IGFS). Insulin-like growth factors are frequently found in bone tissue. Of the two forms, IGF-I and IGF-II, IGF-I is the most powerful form, and it 
exerts its stimulating effect on downstream signalling pathways over different binding proteins.

The opinions in the literature on the exact effect of IGFs on bone regeneration are bivalent. There are in vivo studies showing a positive effect of the IGF system on embryonic and postnatal development of the skeleton 276,277 and on fracture healing 278 280 . Other studies show that IGF-1 functions as a regulator over autocrine activity within the bony callus, thereby stimulating the osteoprogenitor cells at the fracture site 281-284. Furthermore, Weiss and colleagues show that low IGF-1 concentrations are associated with non-union development ${ }^{282}$. On the other hand, some studies demonstrate an increased fracture healing tendency in IGF-I knockout mice ${ }^{285}$ and enhanced IGF-I/II gene expression in mice with non-unions ${ }^{286}$.

\subsubsection{Matrix-metalloproteinases (Mmp)}

Metalloproteinases are enzymes, some of which degrade the extracellular matrix of cartilage and bone. The degradation of the extracellular matrix in newly formed bone enables the ingrowth of blood vessels and subsequent osteogenesis ${ }^{154}$. In various studies, Mmp-9 and Mmp-13 are demonstrated to be relevant for both cartilage and bone remodelling 287,288. Wigner and colleagues even showed that Mmp-9 and Mmp-13 levels in urine have the potential to act as biomarkers for fracture healing ${ }^{289}$.

\subsubsection{Angiogenic factors}

\subsubsection{Hypoxia-inducible factor- $1 \alpha(H I F-1 \alpha)$}

As blood supply is damaged at the fracture site due to the initial trauma, an oxygen debt arises. In such hypoxic environment, the expression of pro-angiogenic factors, which promote angiogenesis during fracture healing, is induced, but also the functions of osteoblasts, osteoclasts and chondrocytes are directly regulated towards cell proliferation. The oxygen-sensitive transcriptional activator hypoxia-inducible factor1 (HIF-1) is a key transcriptional mediator of the response to hypoxic conditions ${ }^{290}$. HIF-1 participates in the process of fracture repair by triggering angiogenesis, facilitating the migration of inflammatory cells, recruiting osteoprogenitor cells and promoting the differentiation of cells in the fracture site. ${ }^{291-293}$. Subunit HIF-1 $\alpha$ expression can be induced by a hypoxic environment with an oxygen concentration of less than $6 \% 294,295$. The promotion of angiogenesis and osteogenesis takes place by upregulating VEGF and other target genes. HIF-1 $\alpha$ further facilitates the migration and proliferation of MSCs by enhancing the expression of SDF-1 and Twist-related protein (TWIST), respectively 296,297.

\subsubsection{Vascular endothelial growth factor (VEGF)}

The VEGF group is formed by the isoforms VEGF-A, -B, -C and -D. ${ }^{290}$ These isoforms are produced by endothelial cells, macrophages, chondrocytes and osteoblasts ${ }^{298,299}$, and their functions are exerted by binding to its receptors VEGFR-1 and VEGFR-2 on the target cells ${ }^{298}$. Those functions are different for every isoform. VEGF-C and -D are closely associated with lymphangiogenesis; the biological significance of VEGF-B, however, is still unclear ${ }^{299}$. The isoform of VEGF-A predominantly regulates angiogenesis and the growth of endothelial cells 299,300. Several studies have demonstrated that VEGF is an essential growth factor in bone healing as it is a key regulator of angiogenesis. Street et al. have found that a high level of VEGF in fracture 
haematoma not only promotes the recruitment of endothelial cells and osteoblasts but also stimulates the biological function of those cells ${ }^{301,302}$. In the intramembranous ossification stage, VEGF induces vascular invasion into the fracture site, providing the nutrients and numerous cells which are required for bone healing 299 . In the endochondral ossification phase, VEGF expressed by hypertrophic chondrocytes promotes vascular invasion into the cartilage zone, accelerates chondrocytes apoptosis and stimulates bone matrix synthesis 303,304 . Animal studies have demonstrated that exogenous VEGF stimulates blood vessel formation and enhances the formation and ossification of a callus, whereas inhibiting the activity of VEGF had the opposite effect 138,305 .

Platelet-derived growth factor (PDGF). Platelet-derived growth factor is mainly synthesised by platelets but can also be set free by other cells, such as macrophages, endothelial cells and smooth muscle cells. This group of growth factors exists in different forms: PDGF-A, PDGF-B, PDGF-C, PDGF-D and PDGF-AB. The last form plays a role in the inflammatory stage of fracture healing and controls the inflammatory response ${ }^{306,307}$. PDGF-AB is upregulated in the early phases of the inflammatory stage, and it has clear angiogenic activity 151,281,308. A positive effect of PDGF-AB on normal bone regeneration has been shown ${ }^{306,308}$, and conforming to those results, low PDGF$\mathrm{AB}$ concentrations were detected in patients with non-unions ${ }^{309}$.

\subsubsection{Chemokines}

The chemokine group, which plays an important role in the recruitment of immune cells into the fracture haematoma, consists of approximately 50 ligands with approximately 20 corresponding receptors 310,311 .

\subsubsection{CC Chemokine ligand (Ccl) 2 and CC Chemokine receptor (Ccr) 2}

In particular, the system of $\mathrm{Ccl} 2$ and $\mathrm{Ccr} 2$ initiates the recruitment of macrophages and mesenchymal cells in the inflammatory stage of fracture healing, but this system also seems to play a role in the mineralisation process of the soft callus ${ }^{312,313}$.

\subsubsection{Chemokine CXCL8 (Interleukin-8 or CXC motif chemokine 8)}

This inflammatory mediator is produced by macrophages, fibroblasts and endothelial and epithelial cells. This chemokine, also identified as a proinflammatory cytokine, is upregulated within the first 24 hours after injury 173,314. It has an essential function in the chemotactic mobilisation of neutrophils, leukocytes and other immune cells ${ }^{315}$ but also has angiogenic and osteoinductive effects ${ }^{316}$.

\subsubsection{CXC chemokine ligand (CXCl) 12 and CXC chemokine receptor (CXCr) 4}

This system also has a role in the recruitment of mesenchymal stem cells into the fracture hematoma 20,220. As chemokine CXCL8, it also seems to contribute to the angiogenesis and callus-forming process and promotion of bone regeneration $21,317,318$.

\subsubsection{Prostaglandins}

Arachidonic acid as a resource for prostaglandins is metabolised by cyclooxygenase (COX) 1 or 2 and prostaglandin synthases. End products of this reaction are different prostaglandins (PGs) and thromboxane A2 (TXA2) ${ }^{319,320}$. Of those prostaglandins, PGE2 appears to increase bone regeneration by enhancing the BMP-2, osteocalcin, receptor 
activator of nuclear factor kappa-B ligand (RANKL), tartrate-resistant acid phosphatase (TRAP) and Mmp9 expression 320,321.

\subsubsection{Other factors}

The arginine-nitric oxide (NO) pathway and the Wnt signalling pathway also play important roles in the development of non-union.

\subsubsection{Arginine-NO pathway}

Another factor which plays an important role in the healing process of patients is their nutritional status. Considering the disastrous effect of malnutrition on bone healing, it is likely that not only endogenous depletion but also exogenous supply with protein and amino acids is vital for good fracture healing. ${ }^{322}$ In the population of patients with fractures, there are two categories of patients who need special attention concerning this factor. First, it has been observed that a malnourished state in elderly patients leads to prolonged recovery, complications such as non-union and increased morbidity and mortality after fractures 139,323,324. Second, nutritional requirements in polytraumatised patients are elevated, and metabolic changes in these patients can lead to the loss of muscle mass, impaired healing, immobility and susceptibility to infections, regardless of the age of the patient ${ }^{325,326}$. As both patient groups are growing due to the ageing of society and an increased survival rate of polytrauma patients, dietary interventions, such as protein- and amino acid suppletion, to avert impaired bone healing could contribute to improved patient outcomes and reduced healthcare costs.

One of the factors associated with a better nutritional state, which influences bone repair, is the arginine-nitric oxide (NO) metabolism. This metabolism is an important factor in angiogenesis and revascularization. In the literature, many studies concerning fracture healing emphasise the key role of revascularization and angiogenesis and its necessity for new bone formation 129-132,139,327-329 because those processes are a prerequisite for inducing cell growth, the distribution of cytokines and the delivery of nutrients and oxygen to the fracture site. If the blood supply during fracture healing is compromised, there is a high probability of developing pseudarthrosis or a non-union 138,330 .

The complex process of neovascularization, vascular reactivity, angiogenesis and the formation of bone cells during fracture healing partly depends on the regulation by nitric oxide $323,331,332$, in addition to other pro- and anti-angiogenic mediators such as VEGF, HIF-1 $\alpha$, FGF, interleukins and BMPs 129,132,139.

In the inflammatory stage of fracture healing, nitric oxide is a well-established mediator which enhances blood flow throughout the fracture site by vasodilation ${ }^{333-335}$. NO is produced in the human body either via the endogenous nitrate-nitrite-nitric oxide pathway ${ }^{336}$ or the classic L-arginine-NO-synthase pathway ${ }^{337}$. The nitrate-nitrite-nitric oxide pathway forms an important alternative to the classic pathway, especially in hypoxic states ${ }^{338}$. In the classic pathway, $\mathrm{NO}$ is produced by the oxidation of L-arginine catalysed by the NOS enzyme family 331,333,337,339. A disturbance of the equilibrium between the three isoforms of NOS (i.e., neuronal NOS [nNOS/NOS-1], inducible NOS [iNOS/NOS-2], endothelial NOS [eNOS/NOS-3]) in this pathway will lead to impaired fracture healing 340 . There is experimental evidence indicating that sufficient production of NO from its precursor arginine is of particular importance for vascular reactivity and the healing of fractures, thereby establishing a possible link between nutritional status and bone healing 322,323,330,341. Wijnands et al. showed that the 
citrulline-arginine-NO metabolism is impaired in atrophic non-unions and arginine and NO levels are increased in hypertrophic non-unions, suggesting that NO is involved in the development of non-unions ${ }^{322}$. Supportive of this theory is the finding that arginine levels decrease in cases of stress, such as wound healing and sepsis, as possible initial circumstances in the pathogenesis of non-union ${ }^{342,343}$. Further, in vivo studies have demonstrated that the upregulation of NOS expression and thus of NO production encouraged fracture healing ${ }^{331,344}$. Moreover, evidence has shown that NO can enhance the conversion of arginine into ornithine by arginase enzyme, increasing the production of polyamine and proline, which are considered to be substrates for collagen synthesis 345 . This could be considered another explanation to clarify the importance of arginineNO metabolism in fracture healing.

The NOS enzymes. The NOS enzymes are subdivided into three isoforms in humans: neuronal nitric oxide synthase (nNOS or NOS1), inducible nitric oxide synthase (iNOS or NOS2), and endothelial nitric oxide synthase (eNOS or NOS3) ${ }^{346}$. The iNOS enzyme is $\mathrm{Ca}^{2+}$-independent, which produces a large amount of NO under inflammatory conditions and is mainly expressed in macrophages. The $\mathrm{Ca}^{2+}$-dependent enzymes, eNOS and nNOS, are constitutively expressed in endothelial and neuronal cells, respectively, with low-output NO production ${ }^{346}$. During fracture repair, there seems to be an appropriate NOS isoform expression equilibrium between NOS isoforms, especially in the early stages of fracture healing, which is very important for a normal course of callus formation and bone stability after fracture 331,333,334,339. The high output of NO generated by iNOS is considered a likely 'damaging NO' to bone healing as it would increase the osteoclastic bone resorption and reduce the proliferation of osteoblasts but promote the apoptosis of osteoblasts. Conversely, the low output of NO produced by eNOS is considered a 'protective NO', which has the opposite effect compared with that produced by iNOS. It has been demonstrated that the NO produced by eNOS is a key factor in regulating vascular tone ${ }^{347}$. Increasing the activity of eNOS may not only directly upregulate the function of osteoclasts and osteoblasts but also promote angiogenesis ${ }^{348}$. Early upregulation of the NOS enzymes, which mediate the conversion of arginine into citrulline and NO, is associated with a normal inflammatory phase of fracture healing ${ }^{333}$. In addition, artificial stimulation of the NOS enzymes results in increased bone stability during recovery after fracture ${ }^{331}$. Disturbances in NO production, either through insufficient NOS activity or depletion of substrate, is associated with an increased incidence of delayed fracture healing and non-union 322,340 .

Arginine as a substrate for NO production. For humans, arginine (or L-arginine) is considered to be a semi-essential amino acid, and the vast amount of arginine in the body is derived from protein breakdown, accounting for approximately $80 \%$ of total arginine. Approximately $10 \%-15 \%$ of arginine is converted from citrulline by the enzymes arginine-succinate synthetase (ASS) and arginine-succinate lyase (ASL) ${ }^{346}$.

From the arginine taken from proteins in food, approximately $40 \%$ is oxidised in the intestines by bacteria and arginases during the first passage 349,350 . Then, $10 \%-15 \%$ of the residual systemic arginine is metabolised during the passage through the liver $349,351,352$. The remaining arginine can be used intracellularly but has to compete with other arginine analogues for the transmembrane carrier protein hCAT-2B ${ }^{339}$, with the result that only $\sim 1 \%$ of the ingested arginine is actually used for NO production ${ }^{353}$. To exemplify these facts on arginine metabolism, Luiking et al. observed that in conditions of sepsis, chronic stress, malnutrition and polytrauma, arginine availability is impaired, 
which may lead to insufficient callus formation and, ultimately, to severe problems in the bone-healing process ${ }^{324,354}$. Further, Wijnands et al. found that arginine deficiency was related to delayed fracture healing or non-union ${ }^{322}$, while, in another study, an improvement of bone formation was observed by supplying arginine silicate inositol complex, indicating that arginine administration might increase bone healing 355 . Moreover, Meesters et al. demonstrated that disturbances of NO production, either through insufficient NOS activity or depletion of substrate, is associated with an increased incidence of delayed fracture healing and non-union ${ }^{322,340}$. (Figure 1.6.)

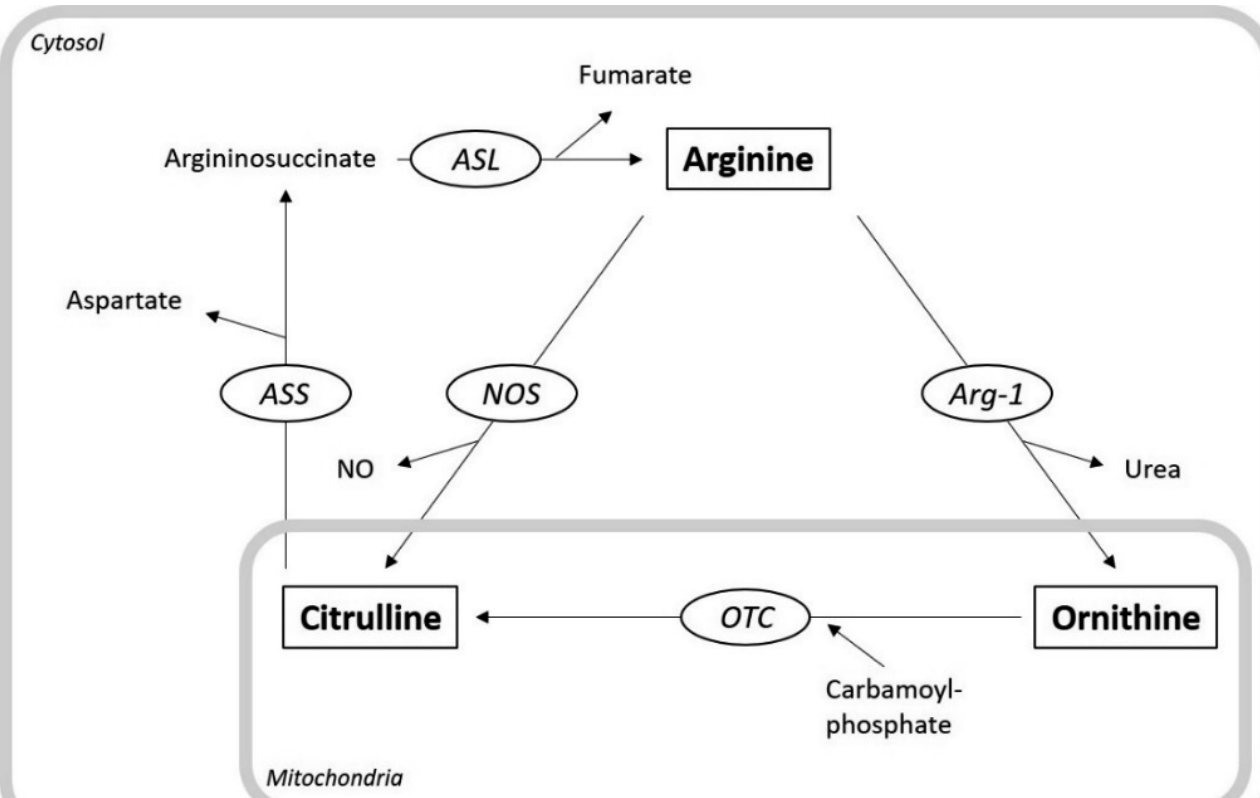

Figure 1.6. Metabolic pathways of arginine. Arginine is converted over two relevant pathways. In one pathway, arginine is converted by Arginase 1 (Arg-1) into urea and ornithine, which are precursors of polyamines and collagens. In the other pathway, arginine is converted by NOS (nitric oxide synthase) into NO (nitric oxide) and citrulline. Citrulline is in turn recycled by argininosuccinate synthetase (ASS) into argininosuccinate and then by argininosuccinate lyase (ASL) into arginine again. Furthermore, ornithine is converted into citrulline by ornithine transcarbamoylase (OTC). [This illustration was created by Martijn Hofman and is licensed under Creative Commons CC-BY-SA 4.0].

Citrulline as a substrate for NO production. Citrulline (C6H13N3O3) is a nonproteinogenic $\alpha$-amino acid which was initially isolated from watermelon (Citrullus lanatus) in 1914 by Koga and Odake ${ }^{356}$. In mammals, citrulline is produced as an intermediary product in the urea cycle in a reaction between carbamoyl phosphate and ornithine catalysed by the enzyme ornithine transcarbamylase. Subsequently, argininosuccinate-synthase (ASS) turns citrulline into argininosuccinate, which is then metabolised into arginine and fumerate by the enzyme ASL ${ }^{333}$. Thus, citrulline acts as a precursor of arginine production. Moreover, citrulline is a secondary product of the conversion of arginine into NO by the NOS enzyme family. As mentioned before, citrulline can be converted back into arginine ${ }^{346,357}$. (Figure 1.6.) 
Citrulline is normally released by the small intestine, converted to arginine in the kidney and released into the circulation. This citrulline-NO pathway appears to be the solution for the inefficient NO production via the L-arginine-NO pathway because the recycling of citrulline into arginine is a very efficient process $339,358-360$. The big advantage of the citrulline-NO pathway over the L-arginine-NO pathway is that the catabolism of orally taken citrulline is restricted in the intestines ${ }^{361}$ and it is not extracted by the liver 339,352 , leaving the bulk of citrulline for conversion, mainly in the kidneys, to arginine $339,352,362$.

Moreover, several studies have demonstrated that, in this way, citrulline supplementation is significantly more potent in increasing the level of

arginine in tissue and plasma in the human body than an equivalent dose of arginine 322,363,364.

In adults, the citrulline converted by the kidney is enough to provide the body's full arginine requirements. Arginine synthesised from citrulline represents $60 \%$ of the de novo arginine synthesis in the organism but only $5 \%$ to $15 \%$ of the circulating arginine 365. Various studies have shown that orally administered citrulline supplementation is well absorbed ${ }^{366-368}$, increases protein synthesis ${ }^{358}$ and restores the arginine pool and nitrogen homeostasis 339,369 . In addition, increased concentrations of citrulline are associated with increased activation of iNOS and eNOS 333,370 and thus have positive effects on NO production and can improve nitrogen homeostasis. Those effects are likely to be mediated through the increased availability of arginine in tissue and plasma 322,339,369. There are several studies demonstrating the beneficial effects of citrulline supplementation on various disorders, such as sickle cell disease ${ }^{371}$, hypertension, high pulmonary artery pressure ${ }^{372}$ and protein malnutrition ${ }^{358,373}$ but also on improving sports performance 374,375 . On the other hand, other authors failed to find biological effects of citrulline supplementation in either animal or human studies. For example, Bouillanne et al. carried out a randomised, multi-centre study to investigate the effects of citrulline supplementation on malnourished elderly patients. They found that oral citrulline supplementation for 3 weeks could not enhance protein synthesis in the whole body or in the liver ${ }^{376}$. Ham and colleagues found that citrulline had no effect on preventing the loss of skeletal muscle in 14 days of hind-limb immobilisation in mice 377. A meta-analysis performed by Mirenayat et al. showed that citrulline supplementation could not decrease blood pressure ${ }^{378}$. Concerning bone healing, Wijnands and colleagues demonstrated a significant change in the concentrations of arginine, citrulline and ornithine in non-union patients compared with healed-fracture patients ${ }^{322}$. More recently, Rajfer and colleagues reported that COMB-4 (a mixture of citrulline, paullinia cupana, ginger and muira puama) accelerates the process of fracture healing in rats by increasing the expression of iNOS ${ }^{331}$. However, because they used a compound in their study, the conclusions are not fully transferable and informative on the effects of citrulline supplementation on its own.

\subsubsection{Wht signalling}

In the canonical Wnt (wingless/integrated) signalling pathway, a secreted glycoprotein passes signals over a membrane receptor into the cell, where the upregulation of $\beta$ catenin levels modulates gene expression. This pathway is shown to be involved in fracture healing in mice 379,380 . 


\subsubsection{Cells involved in the inflammation response}

Cells from the immune system, derived from hematopoietic stem cells, play a highly important role in the process of fracture healing and bone regeneration. A disturbance of the cells or their function results in pathologic fracture healing. The cells are recruited mainly during the first stage of fracture healing, but their effects are not limited to that stage and continue throughout the entire healing process. (Figure 1.7.)

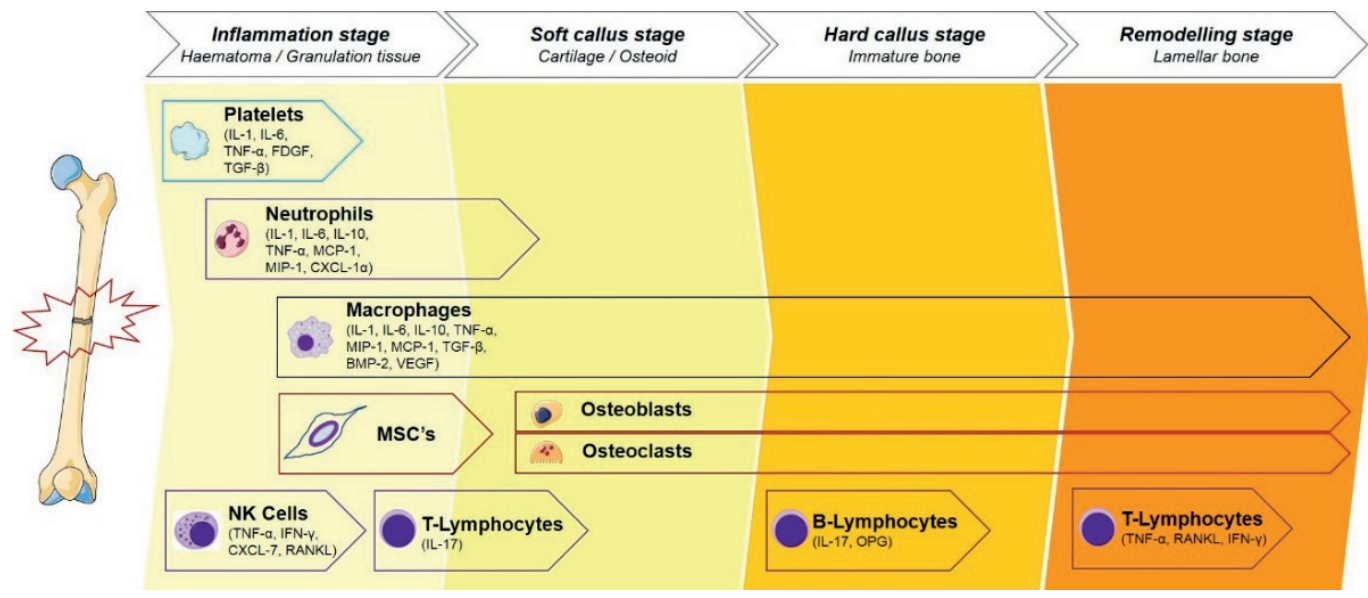

Figure 1.7. Immune cells involved in the fracture healing process. Throughout the four fracture healing stages, different immune cells are active at different time points. [This illustration was adapted by Martijn Hofman (licensed under Creative Commons CC-BY-SA 4.0) using the original illustration from Baht et al. ${ }^{327}$ and images from Smart Servier Medical Art (https://smart.servier.com/)].

\subsubsection{Platelets}

Platelets are immune cells of myeloid origin and, besides their essential function in the construction of fracture haematomas, they also discharge inflammatory cytokines (IL1, IL-6, TNF- $\alpha$ ) and growth factors (PGDF, TGF- $\beta$, VEGF and IGF) ${ }^{381}$ for the mobilisation of neutrophils, monocytes and mesenchymal progenitor cells 382,383. As the understanding of the part growth factors play in the fracture healing process grows, the importance of the fracture haematoma, containing platelets, is emphasised. On the other hand, scientific evidence for a stimulating effect of the application of platelet-rich plasma (PRP) in fracture healing is still inconclusive ${ }^{38}$.

\subsubsection{Neutrophils}

Neutrophils are derived from multipotent haematopoietic stem cells in the bone marrow and belong, just as basophils and eosinophils, to a subspecies of leukocytes called polymorphonuclear leukocytes or granulocytes. Neutrophils are the most abundant immune cells in peripheral blood and the primary first responders to trauma 72,73. As components of the innate immune system, neutrophils possess a genetically programmed defence mechanism, which recognises molecular patterns of offensive pathogens or damaged cells by pattern recognition receptors (PRRs). 
These PRRs identify pathogen-associated molecular patterns (PAMPs), consisting of microbial components, and damage-associated molecular patterns (DAMPs), consisting of cell components ${ }^{384}$. Injury-induced local inflammation activates endothelial and immune cells, which produce different cytokines and chemokines, which pre-activate circulating neutrophils ('priming'). The 'priming' of neutrophils enhances cytotoxic responses evoked by activating agonists, alters the expression and affinity of several specific surface receptors, increases the neutrophil oxidative burst and neutrophil migratory response to chemotactic stimulation and delays neutrophil apoptosis ${ }^{384}$. Besides the direct inflow of neutrophils from the bone marrow and damaged vessels at the fracture site, the neutrophils are attracted to the injury site by different chemotactic signals, produced by activated immune cells and stromal cells. The transmigration across the endothelium into the injured tissue, a process called 'homing', is preceded by the rolling ('slowing down') and subsequent firm adhesion of neutrophils to endothelial cells ${ }^{384}$. At the site of injury, their most crucial attribute is the production of different cytokines to mobilise monocytes differentiating into macrophages ${ }^{327}$. Furthermore, they play a considerable role in the development but also the later breakdown of the fibrin thrombus clot at the inflammatory stage of fracture healing. Bastian et al. even suggested that neutrophils synthesise a fibronectin+ extracellular matrix within 48 hours after injury, before stromal cells infiltrate the fracture haematoma ${ }^{385}$. In addition to those functions, neutrophils also remove other redundant cellular and tissue debris at the fracture site 386,387 . The phagocytic function of the neutrophils can be exerted in the following manners ${ }^{384}$.

- Neutrophils bind either with their immunoglobulin receptors to immunoglobulins attached to their targets, or they bind via the $\alpha_{M} \beta_{2}$ integrin (CD11b/CD18 or MAC-1) to particles coated with the complement component C3bi5. Subsequently, the phagocytosis-process is activated.

- Neutrophils can release cytotoxic agents (e.g., proteinases, reactive oxidative and nitric species) by which pathogens or debris are eliminated.

- Neutrophils are able to eliminate pathogens by the discharge of so-called neutrophil extracellular traps (NETs), which are networks of cytotoxic mediators bound to DNA fibres that bind pathogens extracellularly.

On the other hand, the dysregulation of the neutrophil response plays a key role in the origins of various complications after major physiologic stress, such as trauma, burns, ischemic reperfusion injuries and sepsis ${ }^{70,388}$. Shortly after such major stress events, an enhanced and partly undirected immune response, known as systemic inflammatory response syndrome (SIRS), causes an accumulation of neutrophils in different organs throughout the body and a subsequent massive release of cytotoxic factors, damaging the organs and potentially leading to ARDS and MODS 384.

\subsubsection{Macrophages}

Like the neutrophils, macrophages are also phagocytic cells from the myeloid lineage, but they are differentiated from monocytes. In normal healthy bone, these cells are important for bone homeostasis by continuing the balanced bone remodelling. It is achieved by providing a niche for osteoblasts and -clasts and by communication between different bone cells ${ }^{327}$. The role of the macrophages in fracture healing is still the subject of research. As monocytes are attracted to the fracture site by CXCL2, IL-1 and TNF- $\alpha$, they differentiate into macrophages, which are then one of the first types of 
cells to emerge in the fracture haematoma 235. Subsequently, the macrophages differentiate into M1 macrophages triggered by IL-1 or TNF- $\alpha$ or M2 macrophages triggered by IL-4. The M1 macrophages are important in the inflammatory reaction and produce different cytokines (IL-1, IL-6, TNF- $\alpha$, MCP-1, MIP-1 [CXCL2]), which also attract monocytes. They also exert their normal phagocytic function in removing debris and the fibrin thrombus ${ }^{235,389}$. The M2 macrophages appear at a later phase in the inflammatory stage and exert anti-inflammatory activities by producing tissue repair factors, such as IL-10, TGF- $\beta$, BMP- 2 and VEGF, mobilising mesenchymal progenitor cells and initiating osteochondral differentiation and angiogenesis ${ }^{390-392}$.

\subsubsection{Osteoclasts}

Another product of the myeloid cell line is the osteoclast cell, which differentiates directly from monocytes or from macrophages ${ }^{392}$. Triggered by inflammatory signals, osteoclasts can also function as immune cells, and they possess the unique function of the phagocytosis of bone material. The osteoclasts adhere to the cortex of bone and form a ruffled border and a so-called resorption pit. Then, hydrogen ions are secreted to dissolve the hydroxyapatite and lysosomal enzymes are set free to resorb the debris. Together with activated osteoblasts, the osteoclasts organise bone formation or remodelling by resorption of callus tissue, creating bony tunnels for neovascularization or nerve ingrowth ${ }^{393,394}$. This function of osteoclasts is modulated in two ways. The activation of osteoclasts occurs when RANKL, produced by osteoblasts, natural killer (NK) cells or T cells, binds to the osteoclast surface receptor RANK. The deactivation of osteoclasts occurs when osteoprotegerin (OPG), secreted by osteoblasts and B cells, binds to RANK and competes with RANKL binding 327.

\subsubsection{T-Lymphocytes ( $T$ cells) and B-Lymphocytes (B cells)}

Both T cells and B cells are haematopoietic cells of the lymphoid cell line, and both are essential for normal bone homeostasis and adequate fracture healing 242,395. Both cell types exert the most important influence on cell signalling in the inflammatory stage and the hard callus stage ${ }^{396}$. The T cells produce RANKL and thereby activate the phagocytic osteoclasts to break down the thrombus and prepare for the soft callus stage of fracture healing. Furthermore, the cells produce IL-17, which has an immunomodulatory function as described before.

The B cells, on the other hand, produce OPG and thereby inactivate the osteoclasts. They also play a role in the suppression of the inflammatory reaction by inhibiting proinflammatory factors, such as interferon gamma (IFN- $\gamma$ ), TNF- $\alpha$ and IL-2 235,397. Moreover, the $\operatorname{IgM}^{+}-\mathrm{CD} 27^{+}$-memory $\mathrm{B}$ cell complex has been demonstrated to be principally responsible for IL-10 production in fracture healing 397.

\subsubsection{Natural killer (NK) cells}

Like T and B cells, NK cells are haematopoietic cells from the lymphoid lineage, which normally induce apoptosis of hostile or infected cells. In fracture healing, the most obvious role of NK cells, which produce IFN- $\gamma$ and RANKL, is the mobilisation of inflammatory cells and osteoclasts. They also secrete CXCL 7 at a later stage of fracture healing and thereby also have a part in the role mesenchymal progenitor cells play at the fracture site 398,399 . 


\subsubsection{Mesenchymal stromal cells (MSCS)}

Mesenchymal stromal cells are cells with multipotent differentiation capacity which exert an anti-inflammatory response to inflammatory cytokines, described with the term 'licensing' ${ }^{400}$. The licensing is provoked, for instance, by TNF- $\alpha$ and a combination of IL- $1 \alpha$, IL1 $\beta$ and IFN- $\gamma{ }^{401}$. Furthermore, MSCs can also have an immunosuppressive effect, initiated by IL-17 or IFN- $\gamma$ 402,403.

\subsubsection{Communication between cells in the inflammation response}

In general, there are three methods of intercellular communication between the cells of the immune system. The main interaction between cells occurs via direct membrane contact. Soluble mediators can also transfer information from one cell to another. Some of those mediators are described in our introduction, and they include cytokines, chemokines, hormones and nitric oxide. In recent years, a third method of communication between cells has come to the fore. In that method of intercellular communication, small vesicles loaded with different proteins, mRNAs and miRNAs are budded directly from the cell surface membrane itself (ectocytosis) or from an intracellular membrane (exocytosis) in certain physiological or pathological conditions. In general, three groups of vesicles have been identified: exosomes, which are $40 \mathrm{~nm}-$ $100 \mathrm{~nm}$ in size and are produced in intracellular multi-vesicular bodies; micro-vesicles, which are $100 \mathrm{~nm}-1000 \mathrm{~nm}$ in size and are produced by outward budding from the plasma membrane; and apoptotic bodies, which are $500 \mathrm{~nm}-4000 \mathrm{~nm}$ in size and are produced by blebbing from the plasma membrane in apoptotic cells. In the literature, many different names are randomly circulated for these vesicles (micro-particles, exosomes, ectosomes, nano-vesicles, etc.); however, the function of modulating cell functions through transferring their content is more or less the same ${ }^{404,405}$. Further, the lipid bilayer of the vesicles provides stable physical and chemical properties, which protect the incorporated substances from degradation 406 . With respect to bone regeneration, the vesicles are shown to exert their influence mainly through the regulation of osteoblast proliferation and apoptosis. In the early phases of fracture healing, the proliferation of osteoblasts is upregulated and further bone formation depends to a great extent on the prevention of osteoblast apoptosis 405,407. To exert certain effects on processes in the human body, such as the induction of bone formation, micro-vesicles can be loaded with cells or coated with bioactive agents 408,409 . In that respect, Orth et al. were able to show that VEGF-loaded micro-vesicles improved the healing tendency of non-unions in vivo ${ }^{410}$. Zhang et al. demonstrated that microvesicles derived from umbilical cord mesenchymal stem cells improved angiogenesis and fracture healing in vivo ${ }^{411}$. Other authors have also shown positive influences of different micro-vesicles on bone metabolism, osteogenesis and angiogenesis in both in vivo and in vitro experiments ${ }^{412-414}$.

\subsubsection{The neurohumoral response in fracture healing}

Inflammation is the physiological response of an organism to any injury affecting its integrity. It applies both to primary systemic conditions, such as sepsis and polytrauma, and to local trauma such as fractures. As described above, the inflammatory response is regulated by multiple processes. Therefore, neurohumoral systems also play a crucial role in the response as they provide an interactive communication network in addition to various local effects. In case of a systemic or a local trauma, the 
homeostasis of the organism is disturbed, and the readjustment of the homeostasis is regulated and can be influenced by the modulation of the neurohumoral systems.

Neurohumoral modulation of fracture healing is suggested by several studies, which support the observation of accelerated fracture healing in patients with concomitant traumatic brain injury. All of the studies suggest an underlying neurohormonal or neurohumoral mechanism as a cause for the improved fracture healing in the patients 93,415-417. Circulating humoral factors released by the damaged brain and direct neural pathways are both thought to be partly responsible 97,418 .

It is generally recognised that the central and peripheral nervous systems affect the immune system and vice versa ${ }^{419}$. That bi-directional communication increases the efficiency of both systems in the inflammatory response to injury.

In case of an injury, sensory information is sent from the site of injury to the cerebral cortex. Subsequently, the activated hypothalamic nuclei stimulate different neurohumoral pathways, which then expose the target organs to a cocktail of ligands, which influence the local angiogenesis, inflammation and immune reactions and coordinate the metabolic changes in situ 420 .

Briefly, both humoral and neuronal mediators contribute to the regulation of the inflammatory response to injury. Humoral mediators reach their target cells in distant organs by diffusion or transport by blood flow. Substances which are released by nerves (e.g., norepinephrine and acetylcholine) rapidly reach specific cell groups of distant organs ${ }^{421}$.

The four neurohumoral pathways, which are well represented in the literature, are the autonomic nervous system, the Hypothalamic-Pituitary-Adrenal axis (HPA) system, the neuroendocrine system and the Renin-Angiotensin-Aldosterone System (RAAS). (Figure 1.8.) However, the main focus in research is on the influence of these systems on systemic diseases and conditions, such as cardiovascular diseases, sepsis and polytrauma, and only a negligible number of studies address the influence of these systems on fracture healing and bone regeneration.

\subsubsection{The autonomic nervous system}

The autonomic nervous system exerts a direct regulation of the bone marrow cellularity and especially of the lymphoid tissue. Lymphocytes in turn express different receptors for many neurotransmitters, neurohormones and neurohumors, such as steroids, catecholamines, enkaphalines, endorphins, vasoactive peptides and substance P. The denervation of bone causes an impaired cellularity of the marrow and impairs the transport of osteoprogenitor cells into the circulation ${ }^{422}$.

The autonomic nervous system, with its parasympathetic and sympathetic division, plays an important role in the regulation of the inflammatory response of the body to bone injury.

The parasympathetic nervous system. The parasympathetic nervous pathway plays an important role in the anti-inflammatory response to injury. The sensory input created by an injury reaches the brainstem via the afferent vagus nerve, where the signals are transmitted via efferent nerves and the release of the parasympathetic neurotransmitter acetylcholine to the different organs occurs 423. Subsequently, acetylcholine reduces the release of various proinflammatory cytokines, such as TNF$\alpha$, IL-1 $\beta$, and IL-6, by 50\%-75\% 421,424,425. This cholinergic anti-inflammatory pathway 
is a regulatory mechanism for controlling the proinflammatory and immune response to injury 426,427 .

In addition to its role as a neurotransmitter of the parasympathetic nervous system, acetylcholine is also located as a paracrine and autocrine signalling molecule in various non-neuronal cells, such as endothelial, mesothelial, epithelial, immunocompetent and smooth muscle cells. In those cells, acetylcholine regulates basic cell functions, including proliferation, differentiation and intercellular communication ${ }^{428}$.

Moreover, osteoblasts have nicotinic acetylcholine receptors (nAChR), which are upregulated during fracture healing after exposition to BMP-2, and those cells express different important enzymes for the synthesis of acetylcholine ${ }^{429}$. By binding to nicotinic receptors expressed by fibroblasts, acetylcholine can modulate the biomechanical aspects of newly formed bone, as those cells can upregulate the collagen expression 429 .

Through binding to the other muscarinic receptor, acetylcholine exerts a vasodilative effect and enhances bone perfusion in the course of fracture healing because the muscarinic receptor stimulates the synthesis of nitric oxide (eNOS) ${ }^{430}$.

Kliemann and colleagues also showed that acetylcholine has a positive effect on fracture healing by improving the cancellous bone microarchitecture, the mechanical strength and the bone matrix synthesis 431 .

In case of a fracture, Prasad et al. and Udupa showed that the first neurotransmitter which increases immediately after injury is acetylcholine. Subsequently, acetylcholine stimulates the adrenal medulla to produce catecholamines, and so the parasympathetic nervous system has a clear interaction with the sympathetic nervous system ${ }^{432,433}$.

The sympathetic nervous system. The hypothalamus-sympathetic nervous system pathway influences the regeneration of bone through various neuromediators, such as neurotransmitters, neurotrophins and neuropeptides. ${ }^{422}$ That influence is possible because of a good innervation of bones with sympathetic and sensory nerves, which are especially represented in the periosteum, around blood vessels within the bone tissue and around osteogenic cells 434,435 . Several studies have shown that if that sympathetic innervation is absent, bone metabolism is disturbed 436,437 . For instance, the perfusion of all organs is modulated swiftly by the sympathetic discharge of catecholamines, whereby adrenaline mainly causes systemic effects and noradrenaline mainly causes local effects. The intended vasoactive effect, vasodilation or vasoconstriction is executed by means of the activation of various receptors 420 , which have been demonstrated by several studies to be present in mammalian bone cells 438,439 . That presumed influence of the sympathetic system on bone regeneration is further reinforced by the findings of Bjurholm et al. and Oshima et al. ${ }^{440,441}$. Furthermore, Grills and colleagues showed an enhanced level of catecholamines (noradrenaline and adrenaline) in callus tissue and an increased biomechanical strength of healing bone after the application of nerve growth factor at the fracture site in an in vivo experiment, showing an increased innervation of callus tissue ${ }^{442}$. In addition to the stimulation of sympathetic innervation, the application of nerve growth factor also has a stimulating effect on the axonal growth of sensory nerve fibres ${ }^{437}$, which play a role in both angiogenesis and osteogenesis during fracture healing through their neurotransmitters CGRP and substance P 112,435. The sympathetic nervous system and the sensory nerves are thought to have an opposite effect on bone metabolism. The sympathetic nervous system has a rather catabolic effect on bone metabolism by increasing the bone 
resorption by osteoclasts and decreasing the bone formation by osteoblasts ${ }^{443}$. That finding is strengthened by studies which show a positive effect of $\beta$-adrenergic receptor blockage on the bone mass density in postmenopausal women, thereby decreasing the risk of osteoporotic fractures ${ }^{444}$. CGRP and substance $\mathrm{P}$, as neurotransmitters of the sensory nerves, rather have the opposite effects to $\beta$-adrenergic receptor blockage on osteoclasts and osteoblasts, causing an anabolic effect on bone metabolism ${ }^{443}$.

In patients with long bone fractures and concomitant traumatic brain injury in which increased bone healing is presumed, a relative inhibition of the sympathetic nervous system via neuromediators in the hypothalamus could be a possible explanation for the phenomenon. That inhibition instigates the mobilisation of mesenchymal stem cells and osteoprogenitor cells in the systemic blood pool, which could induce enhanced fracture healing ${ }^{422}$.

The neurotransmitters of the sympathetic nervous system can also exert an influence on the innate immune system via $\alpha$ - or $\beta$-adrenergic receptors, which are expressed on various cells of the immune system ${ }^{445,446}$. By way of the interaction of catecholamines with the $\alpha$-adrenergic receptors, macrophages are stimulated to discharge TNF- $\alpha$ and thereby exert a proinflammatory effect 447 . The stimulation of the $\beta$-adrenergic receptors causes diminished IL-1 and TNF- $\alpha$ releases and increased IL-10 releases by macrophages and so has an opposite anti-inflammatory effect 448,449 .

\subsubsection{The neuroendocrine system}

The neuroendocrine system is made up of special cells, which respond to signals from the autonomic nervous system by producing and releasing different hormones. Nearly every organ of the human body contains such neuroendocrine cells, and the neuroendocrine system plays an important role, amongst others, in the growth and remodelling of bone tissue. Examples of neurohormones and neurohumors which play a role in the homeostasis of bone tissue are growth hormone (GH), parathyroid hormone (PTH), follicle stimulating hormone (FSH), thyroid hormones, testosterone, oestrogen, vasopressin, angiotensin, neuropeptide $Y$, noradrenaline, adrenaline, insulin growth factor (IGF), and vitamins A and D. Even the bone tissue itself produces hormones, which perform important functions in the mineralisation process of bone matrix, such as osteocalcin and fibroblast growth factor 23 (FGF23) 450,451.

In a study on bone healing in patients with concomitant brain injury, Khallaf and colleagues were able to confirm the stimulating effects on bone healing of some neurohormones (i.e., parathyroid hormone, growth hormone, noradrenaline and adrenaline) and the anti-osteogenic effects of the neurohormone produced in the adrenal cortex (i.e., glucocorticoids) ${ }^{422}$.

Furthermore, the role of histamine is discussed in the literature in connection with this topic because the long-term inhibition of histamine could lead to increased bone resorption and decreased bone formation and, therefore, to an increased fracture risk 452 .

In addition to the hormones produced throughout the human body, cytokines also play a major role in activating different neuro-humoral pathways, such as the sympathetic nervous system and the HPA system 453,454 .

Cytokines, which are released after an injury, can activate multiple areas in the brain. The hypothalamus, the rostroventral medulla and the locus coeruleus can be activated via afferents of the vagal nerve, but the hypothalamus can also be activated by inflammatory molecules released by stimulated perivascular cells of the blood brain 
barrier. Further, cytokines can also activate circumventricular organs without a blood brain barrier such as the area postrema. Subsequently, those activated areas of the brain can activate the sympathetic nervous system and the HPA pathway.

\subsubsection{The hypothalamic-pituitary-adrenal (HPA) system}

After activation of the hypothalamic nuclei via circulating cytokines, vagal stimulation or central stimulation over the postcentral gyrus, the hypothalamus expresses corticotropin releasing hormone ( $\mathrm{CRH})$ and antidiuretic hormone (ADH), also called arginine vasopressin (AVP). CRH stimulates the anterior pituitary gland or adenohypophysis to produce adrenocorticotropic hormone (ACTH), which in turn stimulates the release of glucocorticoids and mineralocorticoids in the adrenal cortex 454. Cortisol exerts mainly anti-inflammatory effects by stimulating the synthesis of the anti-inflammatory cytokines IL-4 and IL-10 and by the downgrading of nuclear factorkappa B (NF- $\mathrm{B}$ ) as an important transcription factor. Moreover, in the hypothalamus and the hypophysis, the prohormone pro-opiomelanocortin (POMC) is produced as a precursor to both adrenocorticotropin hormone (ACTH) and alpha-melanocytestimulating hormone $(\alpha-\mathrm{MSH})$. The latter hormone also has anti-inflammatory activity by suppressing NF- $\kappa B$ and stimulating the discharge of IL-10 455.

The importance of $\alpha$-MSH is mainly known from studies concerning the inflammatory response in septic shock 456 and systemic immune responses ${ }^{457}$, but it can also affect bone metabolism directly by increasing bone turnover 458,459 .

AVP produced in the hypothalamus plays a role in the stimulation of the ACTH release from the anterior pituitary gland, but it also stimulates the posterior pituitary gland or neurohypophysis to release vasopressin to the kidneys and oxytocin to the breasts and uterus 454 .

Another way to activate the HPA pathway, which has been demonstrated in various clinical studies, is via the direct stimulation of the HPA system by cytokines, which in turn stimulates the release of anti-osteogenic cortisol 460.

The importance of the HPA pathway in fracture healing is further emphasised by the findings of Khallaf et al. in their study of patients with long bone fractures and concomitant brain injury. They found normal levels of corticosteroids in the patients as an expression of the neuronal inhibition of the adrenal cortex and of its anti-osteogenic corticosteroid production, inducing a normal inflammatory response to

fracture ${ }^{422}$. In addition to these effects on neuro-hormones, the anterior pituitary gland can also stimulate the sympathetic nervous system directly in a stress response.

\subsubsection{The renin-angiotensin-aldosterone system (RAAS)}

The systemic effects of the renin-angiotensin-aldosterone system (RAAS) as a regulating system of blood pressure and of fluid and electrolyte homeostasis are well known and described in the literature. Disturbances in this regulatory system can cause a variety of diseases, such as cardiovascular diseases and hypertension 461 , renal diseases 462 and metabolic syndrome ${ }^{463}$.

In recent years, evidence has grown to indicate that local skeletal RAAS activity is detrimental for bone metabolism. Those effects are exerted via para- and endocrine stimulation, which induce different pathological implications in the bone ${ }^{464}$. In particular, angiotensin II, the bioactive peptide of the system, could lead to decreased bone formation, bone injuries and osteoporosis 465,466 . 


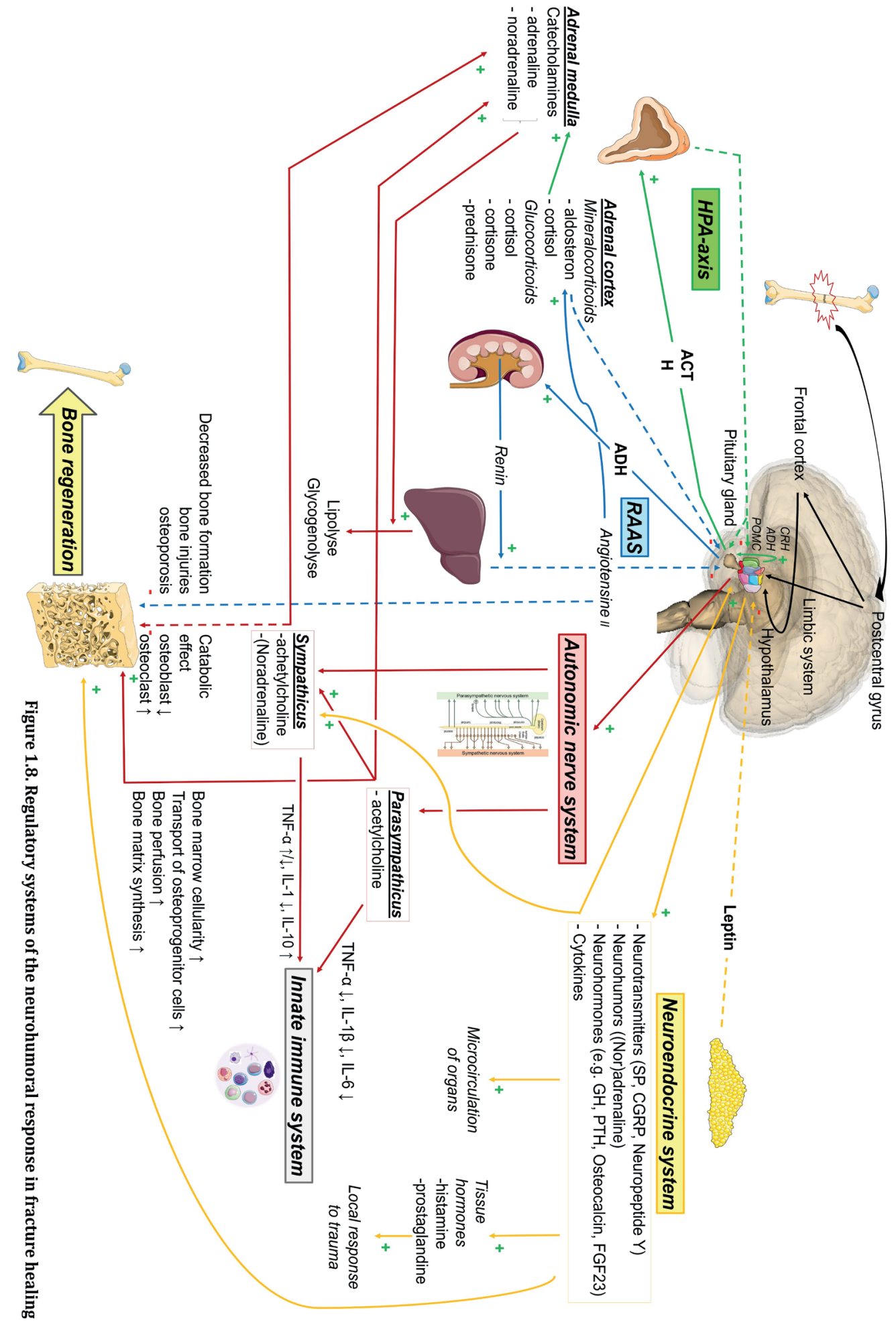


Figure 1.8. Regulatory systems of the neurohumoral response in fracture healing. In case of an injury, sensory information is sent from the site of injury to the cerebral cortex. Subsequently, the hypothalamic nuclei are activated and stimulate various neuro-humoral pathways, which then expose the target organs to a cocktail of ligands, which influence the local angiogenesis, inflammation and immune reactions and coordinate the metabolic changes in situ, thereby influencing the fracture healing process. In this figure, neuro-humoral systems are highlighted: (1) the HPA system (green), (2) the RAA system (blue), (3) the autonomic nervous system (red), and (4) the neuroendocrine system (yellow). [This illustration was created by Martijn Hofman (licensed under Creative Commons CC-BY-SA 4.0) using the original illustrations from Wikimedia commons (https://commons.wikimedia.org/) and images from Smart Servier Medical Art (https://smart.servier.com/)].

Interestingly, the effect of RAAS activity on osteoporosis can be influenced by other therapeutics in osteoporosis treatment. For instance, regular exercise and vitamin D treatment inhibit skeletal RAAS activity and, therefore, osteoporosis development 467,468. On the other hand, cortisol treatment and obesity may enhance tissue RAAS activity and, therefore, increase the risk of osteoporosis 469.

Moreover, several studies have been able to demonstrate that there is an interaction between RAAS activity and the kallikrein-kinin system in which bradykinin plays a decisive role ${ }^{470}$. Other studies have shown that bradykinin negatively influences osteoblast differentiation and enhances osteoclast formation, by which mechanism bone resorption increases and bone mass decreases $471-473$.

Various studies in which RAAS blockers were used have demonstrated such effects in systemic ${ }^{474}$ and bone diseases 464,470 .

\subsection{AIMS AND OUTLINE OF THE PRESENT THESIS}

\subsubsection{Aims of the present thesis}

First of all, the aim of this thesis was to obtain a better understanding of the pathophysiological mechanism of fracture healing in general and of delayed-/nonunion in particular. Another important trigger for initiating this research project was the need to know if the prevailing clinical expert opinion that fracture healing is enhanced in patients with concomitant TBI was based on solid research. Therefore, first, a systematic review of the literature on fracture healing in patients with concomitant TBI was conducted. Second, a retrospective study in which the influence of TBI on the healing of long bone fractures was investigated was performed (Part I).

Through the results of those studies, points of departure were acquired for further research on fracture healing in which neurohumoral pathways play a central role. The influence of various neurohumoral mechanisms were elaborated by clinical studies and several experimental in vivo and in vitro animal studies (Part II).

Following on from the introduction of the present thesis, it has to be concluded that the pathomechanism of the inflammatory response to fracture healing is still a giant puzzle with a lot of missing pieces. The complexity of the regulation mechanisms and the subtle interaction of the neuro-humoral pathways makes it difficult to regulate single modulators of the different pathways in a favourable direction. ${ }^{420}$ That complexity was the inspiration to perform this research project and to compose this thesis in an attempt to find answers to the following questions concerning the neuro-humoral modulation of fracture healing:

1. Does concomitant traumatic brain injury definitely enhance fracture healing? If so, what pathophysiological mechanisms are underlying this effect? (Chapters 2 


\section{and 3)}

2. Does the blockage of the substance P neurokinin-1-receptor influence fracture healing in rats? (Chapter 4)

3. How does the gait pattern and muscle weight evolve during fracture healing in rats? (Chapter 5)

4. Can arginine availability in reamed intramedullary aspirate predict the outcome in non-union healing? (Chapter 6)

5. What is the impact of serum-derived micro-vesicles on osteoblast function after femoral fractures in rats? (Chapter 7)

6. How does neutrophil homeostasis change during the early phases of fracture healing in rats? (Chapter 8)

7. Is the pulmonary neutrophil pool affected by intramedullary stabilised femur fractures in rats? (Chapter 9)

\subsubsection{Outline of the present thesis}

\section{Part I}

The first part of the thesis focusses on the influence of TBI on fracture healing.

In Chapter 2, the results of a systematic review of the influence of concomitant traumatic brain injury on the healing of fractures are described. The aim of that review of the literature was to test the common disseminated opinion that concomitant brain injury enhances bone regeneration and fracture healing. In that review, the evidence provided by both clinical and experimental research throughout the period from 2005 to 2015 was evaluated.

In Chapter 3, the results of a retrospective clinical study we performed to further identify the influence of TBI, chest injury and fracture stabilisation strategy on the development of non-unions in long bone fractures is presented. In that retrospective study, patients with lower extremity long bone fractures were identified and followed until healing of the fractures. Further, concomitant diseases and complications were recorded and the healing of the fractures was assessed according to the bridging of $3 / 4$ cortices on the X-rays and by way of the fracture healing response according to Spencer. The influence of different fracture stabilisation strategies applied in the treatment of long bone fractures were also highlighted. The main outcome parameter was the occurrence of non-union.

\section{Part II}

In the second part of this thesis, the focus will be on the neuro-humoral modulation of fracture healing in the experimental setting of a femur fracture model in rats.

According to the literature, many possible influencing substances have been studied in respect of fracture healing and bone regeneration. Of that immense number of substances, we selected substance $P$ and citrulline for comprehensive critical evaluation. Also, the cellular immune response in fracture healing and the influence of fractures on the neutrophil function was investigated.

In Chapter 4, the effect of a selective blockage of the neurokinin-1-receptor in rats with an intramedullary stabilised femur fracture is described. This blockage eliminates the influence of substance P on the inflammatory response to fracture. In this study, the gene expression of important proteins in the first crucial phases of fracture healing was 
evaluated and the fracture healing process was further assessed with micro-computed tomography (CT) imaging and biomechanical testing up to three months after fracture induction. The primary outcome parameter was biomechanical strength and the secondary outcome parameters were the gene expression and the callus dimensions in the micro-CT evaluation.

In Chapter 5, the influence of an intramedullary stabilised femur fracture on in vivo gait pattern and muscle atrophy in rats is identified. The improvement of mobilisation and gait parameters are very important for the (long-term) clinical outcome of patients after lower extremity fractures. Gait analysis is gaining attention and importance in different medical disciplines. However, in small animal fracture research models, gait analysis is still under-studied. Therefore, in this chapter, we expand on an experimental gait analysis method, the CatWalk ${ }^{\mathrm{TM}}$-system, which we introduced for the first time in gait analyses of a lower extremity fracture in a small animal model.

In Chapter 6, the focus is on the neuro-humoral arginine-NO pathway in fracture healing. In the clinical study reported in this chapter, the predictive value of biomarkers of the arginine-nitric oxide metabolism in trabecular bone harvested during RIA procedures for non-union treatment of long bones was evaluated.

In Chapter 7, the effect of micro-vesicles or microparticles as important links in the intracellular communication on osteoblasts in the inflammatory response of fracture healing is featured. In this in vitro study, the effect of those micro-vesicles was evaluated using a growth assay and quantitative real-time polymerase chain reaction (qRT-PCR).

In Chapter 8, the influence of a femur fracture and its intramedullary treatment on cellular immune response and, more particularly, on neutrophil homeostasis is presented. As dysregulation of the neutrophil response plays a key role in the pathogenesis of inflammatory complications in trauma, we analysed the expression of selectins and integrins on the surface of neutrophils to assess their functionality in the fracture healing process.

In Chapter 9, the pulmonary neutrophil pool is considered in more detail as dysregulation of this pool after trauma is associated with systemic inflammatory response syndrome and the development of acute respiratory distress syndrome. More specifically, the behaviour of the pulmonary neutrophil pool in the first inflammatory phase after femoral fracture and its intramedullary stabilisation was studied.

In Chapter 10, the findings from the studies performed in the context of the thesis are discussed and we present recommendations for possible future research questions, which could build on our findings and could further elucidate the neuro-humoral pathways of the inflammatory response in fracture healing.

In Chapter 11, a summary of the conclusions of the thesis, discussion and future perspectives in English are provided.

\subsection{REFERENCES}

1. Lipphaus A, Witzel U. Finite-Element Syntheses of Callus and Bone Remodeling: Biomechanical Study of Fracture Healing in Long Bones. Anatomical record 2018; 301(12): 2112-21.

2. Liu X, McKenzie JA, Maschhoff CW, Gardner MJ, Silva MJ. Exogenous hedgehog antagonist delays but does not prevent fracture healing in young mice. Bone 2017; 103: 241-51.

3. Donaldson LJ, Reckless IP, Scholes S, Mindell JS, Shelton NJ. The epidemiology of fractures in England. Journal of epidemiology and community health 2008; 62(2): 174-80. 
4. Shi N, Foley K, Lenhart G, Badamgarav E. Direct healthcare costs of hip, vertebral, and non-hip, nonvertebral fractures. Bone 2009; 45(6): 1084-90.

5. Wennergren D, Bergdahl C, Ekelund J, Juto H, Sundfeldt M, Moller M. Epidemiology and incidence of tibia fractures in the Swedish Fracture Register. Injury 2018; 49(11): 2068-74.

6. Kapinos KA, Fischer SH, Mulcahy A, Hayden O, Barron R. Medical Costs for Osteoporosis-Related Fractures in High-Risk Medicare Beneficiaries. Journal of the American Geriatrics Society 2018; 66(12): 2298-304.

7. Wu CH, Tu ST, Chang YF, et al. Fracture liaison services improve outcomes of patients with osteoporosis-related fractures: A systematic literature review and meta-analysis. Bone 2018; 111: 92-100.

8. Burge R, Dawson-Hughes B, Solomon DH, Wong JB, King A, Tosteson A. Incidence and economic burden of osteoporosis-related fractures in the United States, 2005-2025. Journal of bone and mineral research : the official journal of the American Society for Bone and Mineral Research 2007; 22(3): 465-75.

9. Strom O, Borgstrom F, Kanis JA, et al. Osteoporosis: burden, health care provision and opportunities in the EU: a report prepared in collaboration with the International Osteoporosis Foundation (IOF) and the European Federation of Pharmaceutical Industry Associations (EFPIA). Archives of osteoporosis 2011; 6: 59-155.

10. Parziale JR. Disability evaluation of extremity fractures. Physical medicine and rehabilitation clinics of North America 2001; 12(3): 647-57.

11. Collaborators GBDRF. Global, regional, and national comparative risk assessment of 84 behavioural, environmental and occupational, and metabolic risks or clusters of risks, 1990-2016: a systematic analysis for the Global Burden of Disease Study 2016. Lancet 2017; 390(10100): 1345-422.

12. Einhorn TA, Lee CA. Bone regeneration: new findings and potential clinical applications. The Journal of the American Academy of Orthopaedic Surgeons 2001; 9(3): 157-65.

13. Giannoudis PV, Einhorn TA, Marsh D. Fracture healing: the diamond concept. Injury 2007; 38 Suppl 4: S3-6.

14. Edderkaoui B. Potential Role of Chemokines in Fracture Repair. Frontiers in endocrinology 2017; 8: 39.

15. Einhorn TA, Gerstenfeld LC. Fracture healing: mechanisms and interventions. Nature reviews Rheumatology 2015; 11(1): 45-54.

16. Gerstenfeld LC, Cullinane DM, Barnes GL, Graves DT, Einhorn TA. Fracture healing as a post-natal developmental process: molecular, spatial, and temporal aspects of its regulation. Journal of cellular biochemistry 2003; 88(5): 873-84.

17. Mountziaris PM, Mikos AG. Modulation of the inflammatory response for enhanced bone tissue regeneration. Tissue engineering Part B, Reviews 2008; 14(2): 179-86.

18. Oryan A, Monazzah S, Bigham-Sadegh A. Bone injury and fracture healing biology. Biomedical and environmental sciences : BES 2015; 28(1): 57-71.
19. Cho-Chung YS. Autoantibody biomarkers in the detection of cancer. Biochimica et biophysica acta 2006; 1762(6): 587-91.

20. Granero-Molto F, Weis JA, Miga MI, et al. Regenerative effects of transplanted mesenchymal stem cells in fracture healing. Stem cells 2009; 27(8): 1887 98.

21. Kitaori T, Ito H, Schwarz EM, et al. Stromal cellderived factor 1/CXCR4 signaling is critical for the recruitment of mesenchymal stem cells to the fracture site during skeletal repair in a mouse model. Arthritis and rheumatism 2009; 60(3): 813-23.

22. Cho HH, Kyoung KM, Seo MJ, Kim YJ, Bae YC, Jung JS. Overexpression of CXCR4 increases migration and proliferation of human adipose tissue stromal cells. Stem cells and development 2006; 15(6): 853-64.

23. Kon T, Cho TJ, Aizawa $\mathrm{T}$, et al. Expression of osteoprotegerin, receptor activator of NF-kappaB ligand (osteoprotegerin ligand) and related proinflammatory cytokines during fracture healing. Journal of bone and mineral research : the official journal of the American Society for Bone and Mineral Research 2001; 16(6): 1004-14.

24. Lee SK, Lorenzo J. Cytokines regulating osteoclast formation and function. Current opinion in rheumatology 2006; 18(4): 411-8.

25. Yang X, Ricciardi BF, Hernandez-Soria A, Shi Y, Pleshko Camacho N, Bostrom MP. Callus mineralization and maturation are delayed during fracture healing in interleukin-6 knockout mice. Bone 2007; 41(6): 928-36.

26. Corrarino J. Fracture Repair: Mechanisms and Management. . The Journal for Nurse Practitioners 2015; 11(10): 960-7.

27. Goldhahn J, Feron JM, Kanis J, et al. Implications for fracture healing of current and new osteoporosis treatments: an ESCEO consensus paper. Calcified tissue international 2012; 90(5): 343-53.

28. Dimitriou R, Tsiridis E, Giannoudis PV. Current concepts of molecular aspects of bone healing. Injury 2005; 36(12): 1392-404.

29. Kolar P, Gaber T, Perka C, Duda GN, Buttgereit F. Human early fracture hematoma is characterized by inflammation and hypoxia. Clinical orthopaedics and related research 2011; 469(11): 3118-26.

30. Marsell R, Einhorn TA. The biology of fracture healing. Injury 2011; 42(6): 551-5.

31. Brandi ML. How innovations are changing our management of osteoporosis. Medicographia 2010: 1 - 6 .

32. Haverstock BD, Mandracchia VJ. Cigarette smoking and bone healing: implications in foot and ankle surgery. The Journal of foot and ankle surgery: official publication of the American College of Foot and Ankle Surgeons 1998; 37(1): 69-74; discussion 8.

33. Einhorn TA. The cell and molecular biology of fracture healing. Clinical orthopaedics and related research 1998; (355 Suppl): S7-21.

34. Wolff J. Das Gesetz der Transformation der Knochen; 1892.

35. Bassett C. Biophysical principles affecting bone structure. In: Bourne G, ed. Biochemistry and Physiology of bone. New York: Academic Press 1971: 341-76. 
36. Schindeler A, McDonald MM, Bokko P, Little DG. Bone remodeling during fracture repair: The cellular picture. Seminars in cell \& developmental biology 2008; 19(5): 459-66.

37. Carano RA, Filvaroff EH. Angiogenesis and bone repair. Drug discovery today 2003; 8(21): 980-9.

38. Nandra R, Grover L, K P. Fracture non-union epidemiology and treatment. Trauma 2016; 18(1): 3-11.

39. Praemer A, Furner S, Rice D P, Surgeons AAoO. Musculoskeletal conditions in the United States. In: Praemer A, Furner S, P RD, eds. American Academy of Orthopaedic Surgeons. Rosemont; 1999.

40. Mills LA, Aitken SA, Simpson A. The risk of nonunion per fracture: current myths and revised figures from a population of over 4 million adults. Acta orthopaedica $2017 ; \mathbf{8 8}(4)$ : 434-9.

41. Mills LA, Simpson AH. The relative incidence of fracture non-union in the Scottish population $(5.17$ million): a 5-year epidemiological study. BMJ open 2013; $3(2)$.

42. Joshipura M, Mock C, Goosen J, Peden M. Essential Trauma Care: strengthening trauma systems round the world. Injury 2004; 35(9): 841-5.

43. Tzioupis C, Giannoudis PV. Prevalence of long-bone non-unions. Injury 2007; 38 Suppl 2: S3-9.

44. Calori GM, Mazza E, Colombo M, Ripamonti C, Tagliabue L. Treatment of long bone non-unions with polytherapy: indications and clinical results. Injury 2011; 42(6): 587-90.

45. Giannoudis PV, Jones E, Einhorn TA. Fracture healing and bone repair. Injury 2011; 42(6): 549-50.

46. Litrenta J, Tornetta $\mathrm{P}, 3 \mathrm{rd}$, Vallier $\mathrm{H}$, et al. Dynamizations and Exchanges: Success Rates and Indications. Journal of orthopaedic trauma 2015; 29(12): 569-73.

47. A. A. Principles of nonunions In: A. A, ed. Nonunions: Diagnosis, Evaluation and Management Springer-Verlag GmbH; 2018.

48. Tay WH, de Steiger R, Richardson M, Gruen R, Balogh ZJ. Health outcomes of delayed union and nonunion of femoral and tibial shaft fractures. Injury 2014; 45(10): 1653-8.

49. Antonova E, Le TK, Burge R, Mershon J. Tibia shaft fractures: costly burden of nonunions. $B M C$ musculoskeletal disorders 2013; 14: 42.

50. Schottel PC, O'Connor DP, Brinker MR. Time TradeOff as a Measure of Health-Related Quality of Life: Long Bone Nonunions Have a Devastating Impact. The Journal of bone and joint surgery American volume 2015; 97(17): 1406-10.

51. Balogh ZJ, Reumann MK, Gruen RL, et al. Advances and future directions for management of trauma patients with musculoskeletal injuries. Lancet 2012; 380(9847): 1109-19.

52. Kanakaris NK, Giannoudis PV. The health economics of the treatment of long-bone non-unions. Injury 2007; 38 Suppl 2: S77-84.

53. Hak DJ, Fitzpatrick D, Bishop JA, et al. Delayed union and nonunions: epidemiology, clinical issues, and financial aspects. Injury 2014; 45 Suppl 2: S3-7.
54. Wiss DA, Stetson WB. Tibial Nonunion: Treatment Alternatives. The Journal of the American Academy of Orthopaedic Surgeons 1996; 4(5): 249-57.

55. Frolke JP, Patka P. Definition and classification of fracture non-unions. Injury 2007; 38 Suppl 2: S19-22.

56. Zura R, Xiong Z, Einhorn T, et al. Epidemiology of Fracture Nonunion in 18 Human Bones. JAMA surgery 2016; 151(11): e162775.

57. Perumal V, C. R. Factors contributing to nonunion of fractures. Curr Orthopaed 2007; 21: 258-61.

58. Weber BG, O. C. PSEUDARTHROSIS : Pathophysiology Biomechanics Therapy Results. Bern: Hans Huber; 1976.

59. Calori GM, Albisetti W, Agus A, Iori S, Tagliabue L. Risk factors contributing to fracture non-unions. Injury 2007; 38 Suppl 2: S11-8.

60. Calori GM, Phillips M, Jeetle S, Tagliabue L, Giannoudis PV. Classification of non-union: need for a new scoring system? Injury 2008; 39 Suppl 2: S59-63.

61. G. Z. Verzögerte Knochenheilung. In: Biberthaler P, M VG, eds. Knochendefekte und Pseudarthrosen. Deutschland: Springer-Verlag GmbH; 2017: 63-76.

62. Hierholzer C, V. B. Treatment Principles of Nonunion. In: Oestern H, Trentz O, S U, eds. General Trauma Care and Related Aspects Trauma Surgery II. Berlin Heidelberg: Springer-Verlag; 2014: 229-43.

63. Metsemakers WJ, Roels N, Belmans A, Reynders P, Nijs S. Risk factors for nonunion after intramedullary nailing of femoral shaft fractures: Remaining controversies. Injury 2015; 46(8): 1601-7.

64. Hildebrand F, van Griensven M, Huber-Lang M, et al. Is There an Impact of Concomitant Injuries and Timing of Fixation of Major Fractures on Fracture Healing? A Focused Review of Clinical and Experimental Evidence. Journal of orthopaedic trauma 2016; 30(3): 104-12.

65. Dailey HL, Wu KA, Wu PS, McQueen MM, CourtBrown CM. Tibial Fracture Nonunion and Time to Healing After Reamed Intramedullary Nailing: Risk Factors Based on a Single-Center Review of 1003 Patients. Journal of orthopaedic trauma 2018; 32(7): e263-e9.

66. Fischer C, Reiner C, Schmidmaier G, et al. Safety study: is there a pathologic IGF-1, PDGF and TGF-beta cytokine expression caused by adjunct BMP-7 in tibial and femoral non-union therapy? Therapeutics and clinical risk management 2018; 14: 691-7.

67. T. T, PV. G. Pathophysiology of polytrauma. In: Pape H-C, Sanders R, J BJ, eds. The Poly-Traumatized Patient with Fractures A Multi-Disciplinary Approach: Springer; 2011: 33-41.

68. Giannoudis PV, Dinopoulos H, Chalidis B, Hall GM. Surgical stress response. Injury 2006; 37 Suppl 5: S3-9.

69. Aosasa S, Ono S, Mochizuki H, et al. Activation of monocytes and endothelial cells depends on the severity of surgical stress. World journal of surgery 2000; 24(1): $10-6$.

70. Keel M, Trentz 0. Pathophysiology of polytrauma. Injury 2005; 36(6): 691-709.

71. Giannoudis PV. Current concepts of the inflammatory response after major trauma: an update. Injury 2003; 34(6): 397-404. 
72. Botha AJ, Moore FA, Moore EE, Kim FJ, Banerjee A, Peterson VM. Postinjury neutrophil priming and activation: an early vulnerable window. Surgery 1995; 118(2): 358-64; discussion 64-5.

73. Botha AJ, Moore FA, Moore EE, Sauaia A, Banerjee A, Peterson VM. Early neutrophil sequestration after injury: a pathogenic mechanism for multiple organ failure. The Journal of trauma 1995; 39(3): 411-7.

74. T. T. Local inflammatory changes induced by fractures and soft tissue injuries. In: Pape H-C., Sanders $\mathrm{R}, \mathrm{J}$ BJ, eds. The poly-traumatized patient with fractures - a multi-disciplinary approach. Berlin Heidelberg Springer-Verlag; 2011.

75. El Mezayen R, El Gazzar M, Seeds MC, McCall CE, Dreskin SC, MR. N. Endogenous signals released from necrotic cells augment inflammatory responses to bacterial endotoxin. Immunol Lett 2007; 111(1): 36-44.

76. Pugin J. Dear SIRS, the concept of "alarmins" makes a lot of sense! Intensive care medicine 2008; 34(2): 21821.

77. Giannoudis PV, Smith RM, Bellamy MC, Morrison JF, Dickson RA, Guillou PJ. Stimulation of the inflammatory system by reamed and unreamed nailing of femoral fractures. An analysis of the second hit. The Journal of bone and joint surgery British volume 1999; 81(2): 35661.

78. Morley JR, Smith RM, Pape HC, MacDonald DA, Trejdosiewitz LK, Giannoudis PV. Stimulation of the local femoral inflammatory response to fracture and intramedullary reaming: a preliminary study of the source of the second hit phenomenon. The Journal of bone and joint surgery British volume 2008; 90(3): 3939.

79. Probst C, Mirzayan MJ, Mommsen P, et al. Systemic inflammatory effects of traumatic brain injury, femur fracture, and shock: an experimental murine polytrauma model. Mediators of inflammation 2012; 2012: 136020.

80. Dahabreh Z, Dimitriou R, Giannoudis PV. Health economics: a cost analysis of treatment of persistent fracture non-unions using bone morphogenetic protein7. Injury 2007; 38(3): 371-7.

81. Vink R, Nimmo AJ. Novel therapies in development for the treatment of traumatic brain injury. Expert opinion on investigational drugs 2002; 11(10): 1375-86.

82. Hinson HE, Rowell S, Schreiber M. Clinical evidence of inflammation driving secondary brain injury: a systematic review. The journal of trauma and acute care surgery 2015; 78(1): 184-91.

83. Algattas $\mathrm{H}, \mathrm{JH}$ H. Traumatic Brain Injury pathophysiology and treatments: early, intermediate, and late phases post-injury. International journal of molecular sciences 2014; 15(1): 309-41.

84. Boes M, Kain M, Kakar S, et al. Osteogenic effects of traumatic brain injury on experimental fracture-healing. The Journal of bone and joint surgery American volume 2006; 88(4): 738-43.

85. Wei Y, Wang L, Clark JC, Dass CR, Choong PF. Elevated leptin expression in a rat model of fracture and traumatic brain injury. The Journal of pharmacy and pharmacology 2008; 60(12): 1667-72.

86. Gautschi OP, Cadosch D, Frey SP, Skirving AP, Filgueira L, Zellweger R. Serum-mediated osteogenic effect in traumatic brain-injured patients. ANZ journal of surgery 2009; 79(6): 449-55.

87. Cadosch D, Gautschi OP, Thyer M, et al. Humoral factors enhance fracture-healing and callus formation in patients with traumatic brain injury. The Journal of bone and joint surgery American volume 2009; 91(2): 282-8.

88. Zhang D, Zhang P, Wang Y, Han N, Tang C, Jiang B. The influence of brain injury or peripheral nerve injury on calcitonin gene-related peptide concentration variation and fractures healing process. Artificial cells, blood substitutes, and immobilization biotechnology 2009; 37(2): 85-91.

89. Song Y, Bi L, Zhang Z, et al. Increased levels of calcitonin gene-related peptide in serum accelerate fracture healing following traumatic brain injury. Molecular medicine reports 2012; 5(2): 432-8.

90. Lee JS, Ryu CH, Moon NH, Kim SJ, Park SY, Suh KT. Changes in serum levels of receptor activator of nuclear factor-kappaB ligand, osteoprotegerin, IL-6 and TNFalpha in patients with a concomitant head injury and fracture. Archives of orthopaedic and trauma surgery 2009; 129(5): 711-8.

91. Cadosch D, Toffoli AM, Gautschi OP, et al. Serum after traumatic brain injury increases proliferation and supports expression of osteoblast markers in muscle cells. The Journal of bone and joint surgery American volume 2010; 92(3): 645-53.

92. Ozan F, Yildiz H, Bora OA, Pekedis M, Ay Coskun G, Gore 0 . The effect of head trauma on fracture healing: biomechanical testing and finite element analysis. Acta orthopaedica et traumatologica turcica 2010; 44(4): 313-21.

93. Yang TY, Wang TC, Tsai YH, Huang KC. The effects of an injury to the brain on bone healing and callus formation in young adults with fractures of the femoral shaft. The Journal of bone and joint surgery British volume 2012; 94(2): 227-30.

94. Huang W, Li Z, Li Z, Yang R. Does traumatic brain injury result in accelerated mandibular fracture healing? Journal of oral and maxillofacial surgery : official journal of the American Association of Oral and Maxillofacial Surgeons 2012; 70(9): 2135-42.

95. Graef F, Seemann R, Garbe A, et al. Impaired fracture healing with high non-union rates remains irreversible after traumatic brain injury in leptindeficient mice. Journal of musculoskeletal \& neuronal interactions 2017; 17(2): 78-85.

96. Tsitsilonis S, Seemann R, Misch M, et al. The effect of traumatic brain injury on bone healing: an experimental study in a novel in vivo animal model. Injury 2015; 46(4): 661-5.

97. Morley J, Marsh S, Drakoulakis E, Pape HC, Giannoudis PV. Does traumatic brain injury result in accelerated fracture healing? Injury 2005; 36(3): 363-8.

98. Hofman M, Koopmans G, Kobbe P, et al. Improved fracture healing in patients with concomitant traumatic brain injury: proven or not? Mediators of inflammation 2015; 2015: 204842.

99. Wildburger R, Zarkovic N, Egger G, et al. Comparison of the values of basic fibroblast growth factor determined by an immunoassay in the sera of patients with traumatic brain injury and enhanced osteogenesis and the effects of the same sera on the fibroblast growth in vitro. European journal of clinical 
chemistry and clinical biochemistry : journal of the Forum of European Clinical Chemistry Societies 1995; 33(10): 693-8.

100. Khare GN, Gautam VK, Gupta LN, Gupta AK. A new hypothesis for faster healing of fractures in head injured patients. Indian journal of medical sciences 1995; 49(12): 281-4.

101. Cherruau M, Facchinetti P, Baroukh B, Saffar JL. Chemical sympathectomy impairs bone resorption in rats: a role for the sympathetic system on bone metabolism. Bone 1999; 25(5): 545-51.

102. Lerner UH. Neuropeptidergic regulation of bone resorption and bone formation. Journal of musculoskeletal \& neuronal interactions 2002; 2(5): 4407.

103. Jones KB, Mollano AV, Morcuende JA, Cooper RR, Saltzman CL. Bone and brain: a review of neural, hormonal, and musculoskeletal connections. The lowa orthopaedic journal 2004; 24: 123-32.

104. Irie K, Hara-Irie F, Ozawa H, Yajima T. Calcitonin gene-related peptide (CGRP)-containing nerve fibers in bone tissue and their involvement in bone remodeling. Microscopy research and technique 2002; 58(2): 85-90.

105. Santavirta S, Konttinen YT, Nordstrom D, et al. Immunologic studies of nonunited fractures. Acta orthopaedica Scandinavica 1992; 63(6): 579-86.

106. Madsen JE, Hukkanen M, Aune AK, et al. Fracture healing and callus innervation after peripheral nerve resection in rats. Clinical orthopaedics and related research 1998; (351): 230-40.

107. Nordsletten L, Madsen JE, Almaas R, et al. The neuronal regulation of fracture healing. Effects of sciatic nerve resection in rat tibia. Acta orthopaedica Scandinavica 1994; 65(3): 299-304.

108. Apel PJ, Crane D, Northam CN, Callahan M, Smith TL, Teasdall RD. Effect of selective sensory denervation on fracture-healing: an experimental study of rats. The Journal of bone and joint surgery American volume 2009; 91(12): 2886-95.

109. Offley SC, Guo TZ, Wei T, et al. Capsaicin-sensitive sensory neurons contribute to the maintenance of trabecular bone integrity. Journal of bone and mineral research : the official journal of the American Society for Bone and Mineral Research 2005; 20(2): 257-67.

110. Aro H. Effect of nerve injury on fracture healing. Callus formation studied in the rat. Acta orthopaedica Scandinavica 1985; 56(3): 233-7.

111. Onuoha GN. Circulating sensory peptide levels within 24 h of human bone fracture. Peptides 2001; 22(7): 1107-10.

112. Aoki M, Tamai K, Saotome K. Substance P- and calcitonin gene-related peptide-immunofluorescent nerves in the repair of experimental bone defects. International orthopaedics 1994; 18(5): 317-24.

113. Niedermair T, Kuhn V, Doranehgard F, et al. Absence of substance $P$ and the sympathetic nervous system impact on bone structure and chondrocyte differentiation in an adult model of endochondral ossification. Matrix biology : journal of the International Society for Matrix Biology 2014; 38: 22-35.

114. Kan L, Lounev VY, Pignolo RJ, et al. Substance P signaling mediates BMP-dependent heterotopic ossification. Journal of cellular biochemistry 2011; 112(10): 2759-72.

115. Wang L, Zhao R, Shi X, et al. Substance P stimulates bone marrow stromal cell osteogenic activity, osteoclast differentiation, and resorption activity in vitro. Bone 2009; 45(2): 309-20.

116. Lerner UH, Persson E. Osteotropic effects by the neuropeptides calcitonin gene-related peptide, substance $\mathrm{P}$ and vasoactive intestinal peptide. Journal of musculoskeletal \& neuronal interactions 2008; 8(2): 15465.

117. Spector JA, Luchs JS, Mehrara BJ, Greenwald JA, Smith LP, Longaker MT. Expression of bone morphogenetic proteins during membranous bone healing. Plastic and reconstructive surgery 2001; 107(1): 124-34.

118. Lewen A, Soderstrom S, Hillered L, Ebendal T. Expression of serine/threonine kinase receptors in traumatic brain injury. Neuroreport 1997; 8(2): 475-9.

119. Heldin CH, Miyazono K, ten Dijke P. TGF-beta signalling from cell membrane to nucleus through SMAD proteins. Nature 1997; 390(6659): 465-71.

120. Sakou T. Bone morphogenetic proteins: from basic studies to clinical approaches. Bone 1998; 22(6): 591603.

121. Bentz H, Nathan RM, Rosen DM, et al. Purification and characterization of a unique osteoinductive factor from bovine bone. The Journal of biological chemistry 1989; 264(34): 20805-10.

122. Hammonds RG, Jr., Schwall R, Dudley A, et al. Boneinducing activity of mature BMP-2b produced from a hybrid BMP-2a/2b precursor. Molecular endocrinology 1991; 5(1): 149-55.

123. Yang LJ, Jin Y. Immunohistochemical observations on bone morphogenetic protein in normal and abnormal conditions. Clinical orthopaedics and related research 1990; (257): 249-56.

124. Yamada Y, Ueda M, Hibi H, Nagasaka T. Translational research for injectable tissue-engineered bone regeneration using mesenchymal stem cells and platelet-rich plasma: from basic research to clinical case study. Cell transplantation 2004; 13(4): 343-55.

125. Opydo-Chanek M. Bone marrow stromal cells in traumatic brain injury (TBI) therapy: true perspective or false hope? Acta neurobiologiae experimentalis 2007; 67(2): 187-95.

126. Nauth A, Giles E, Potter BK, et al. Heterotopic ossification in orthopaedic trauma. Journal of orthopaedic trauma 2012; 26(12): 684-8.

127. Renfree KJ, Banovac K, Hornicek FJ, Lebwohl NH, Villanueva PA, Nedd KJ. Evaluation of serum osteoblast mitogenic activity in spinal cord and head injury patients with acute heterotopic ossification. Spine 1994; 19(7): 740-6.

128. Bahney CS, Hu DP, Miclau T, 3rd, Marcucio RS. The multifaceted role of the vasculature in endochondral fracture repair. Frontiers in endocrinology 2015; 6: 4.

129. Guo X, Zheng Q, Kulbatski I, et al. Bone regeneration with active angiogenesis by basic fibroblast growth factor gene transfected mesenchymal stem cells seeded on porous beta-TCP ceramic scaffolds. Biomedical materials 2006; 1(3): 93-9. 
130. Kanczler JM, Oreffo RO. Osteogenesis and angiogenesis: the potential for engineering bone. European cells \& materials 2008; 15: 100-14.

131. Minkwitz S, Fassbender M, Kronbach Z, Wildemann B. Longitudinal analysis of osteogenic and angiogenic signaling factors in healing models mimicking atrophic and hypertrophic non-unions in rats. PloS one 2015; 10(4): e0124217.

132. Glowacki J. Angiogenesis in fracture repair. Clinical orthopaedics and related research 1998; (355 Suppl): S82-9.

133. Akhavani MA, Sivakumar B, Paleolog EM, Kang N. Angiogenesis and plastic surgery. Journal of plastic, reconstructive \& aesthetic surgery : JPRAS 2008; 61(12): 1425-37.

134. Jain RK. Molecular regulation of vessel maturation. Nature medicine 2003; 9(6): 685-93.

135. Risau W. Mechanisms of angiogenesis. Nature 1997; 386(6626): 671-4.

136. Chim SM, Tickner J, Chow ST, et al. Angiogenic factors in bone local environment. Cytokine \& growth factor reviews 2013; 24(3): 297-310.

137. Saran U, Gemini Piperni S, Chatterjee S. Role of angiogenesis in bone repair. Archives of biochemistry and biophysics 2014; 561: 109-17.

138. Ogilvie CM, Lu C, Marcucio R, et al. Vascular endothelial growth factor improves bone repair in a murine nonunion model. The Iowa orthopaedic journal 2012; 32: 90-4.

139. Gruber R, Koch H, Doll BA, Tegtmeier F, Einhorn TA, Hollinger J0. Fracture healing in the elderly patient. Experimental gerontology 2006; 41(11): 1080-93.

140. Burkhardt R, Kettner G, Bohm W, et al. Changes in trabecular bone, hematopoiesis and bone marrow vessels in aplastic anemia, primary osteoporosis, and old age: a comparative histomorphometric study. Bone 1987; 8(3): 157-64.

141. Fang TD, Salim A, Xia W, et al. Angiogenesis is required for successful bone induction during distraction osteogenesis. Journal of bone and mineral research : the official journal of the American Society for Bone and Mineral Research 2005; 20(7): 1114-24.

142. Li G, Simpson AH, Kenwright J, Triffitt JT. Effect of lengthening rate on angiogenesis during distraction osteogenesis. Journal of orthopaedic research : official publication of the Orthopaedic Research Society 1999; 17(3): 362-7.

143. Hausman MR, Schaffler MB, Majeska RJ. Prevention of fracture healing in rats by an inhibitor of angiogenesis. Bone 2001; 29(6): 560-4.

144. Kelly PJ, Montgomery RJ, Bronk JT. Reaction of the circulatory system to injury and regeneration. Clinical orthopaedics and related research 1990; (254): 275-88.

145. Gordillo GM, Sen CK. Revisiting the essential role of oxygen in wound healing. American journal of surgery 2003; 186(3): 259-63.

146. Brighton CT, Hunt RM. Early histological and ultrastructural changes in medullary fracture callus. The Journal of bone and joint surgery American volume 1991; 73(6): 832-47.

147. Rajpurohit R, Koch CJ, Tao Z, Teixeira CM, Shapiro IM. Adaptation of chondrocytes to low oxygen tension: relationship between hypoxia and cellular metabolism. Journal of cellular physiology 1996; 168(2): 424-32.

148. Brandi ML, Collin-Osdoby P. Vascular biology and the skeleton. Journal of bone and mineral research : the official journal of the American Society for Bone and Mineral Research 2006; 21(2): 183-92.

149. Clines GA. Prospects for osteoprogenitor stem cells in fracture repair and osteoporosis. Current opinion in organ transplantation 2010; 15(1): 73-8.

150. Dimitriou R, Jones E, McGonagle D, Giannoudis PV. Bone regeneration: current concepts and future directions. BMC medicine 2011; 9: 66.

151. Devescovi V, Leonardi E, Ciapetti G, Cenni E. Growth factors in bone repair. La Chirurgia degli organi di movimento 2008; 92(3): 161-8.

152. Hoff $\mathrm{P}$, Maschmeyer $\mathrm{P}$, Gaber $\mathrm{T}$, et al. Human immune cells' behavior and survival under bioenergetically restricted conditions in an in vitro fracture hematoma model. Cellular \& molecular immunology 2013; 10(2): 151-8.

153. Zhang T, Yao Y. Effects of inflammatory cytokines on bone/cartilage repair. Journal of cellular biochemistry 2018.

154. Ding ZC, Lin YK, Gan YK, Tang TT. Molecular pathogenesis of fracture nonunion. Journal of orthopaedic translation 2018; 14: 45-56.

155. Waki T, Lee SY, Niikura T, et al. Profiling microRNA expression during fracture healing. BMC musculoskeletal disorders 2016; 17: 83.

156. Waki T, Lee SY, Niikura T, et al. Profiling microRNA expression in fracture nonunions: Potential role of microRNAs in nonunion formation studied in a rat model. The bone \& joint journal 2015; 97-B(8): 1144-51.

157. Dimitriou R, Carr IM, West RM, Markham AF, Giannoudis PV. Genetic predisposition to fracture nonunion: a case control study of a preliminary single nucleotide polymorphisms analysis of the BMP pathway. BMC musculoskeletal disorders 2011; 12: 44.

158. Sathyendra V, Donahue HJ, Vrana KE, et al. Single Nucleotide Polymorphisms in Osteogenic Genes in Atrophic Delayed Fracture-Healing: A Preliminary Investigation. The Journal of bone and joint surgery American volume 2014; 96(15): 1242-8.

159. Pensak MJ, Lieberman JR. Gene therapy for bone regeneration. Current pharmaceutical design 2013; 19(19): 3466-73.

160. Cao H, Sun ZB, Zhang L, et al. Adenovirus-mediated bone morphogenetic protein-2 promotes osteogenic differentiation in human mesenchymal stem cells in vitro. Experimental and therapeutic medicine 2017; 14(1): 377-82.

161. Munsell EV, Kurpad DS, Freeman TA, Sullivan MO. Histone-targeted gene transfer of bone morphogenetic protein-2 enhances mesenchymal stem cell chondrogenic differentiation. Acta biomaterialia 2018; 71: 156-67.

162. Ali S, Hussain SR, Singh A, et al. Study of CysteineRich Protein 61 Genetic Polymorphism in Predisposition to Fracture Nonunion: A Case Control. Genetics research international 2015; 2015: 754872.

163. Zeckey C, Hildebrand F, Glaubitz LM, et al. Are polymorphisms of molecules involved in bone healing correlated to aseptic femoral and tibial shaft non- 
unions? Journal of orthopaedic research : official publication of the Orthopaedic Research Society 2011; 29(11): 1724-31.

164. Zimmermann G, Schmeckenbecher KH, Boeuf S, et al. Differential gene expression analysis in fracture callus of patients with regular and failed bone healing. Injury 2012; 43(3): 347-56.

165. Ball AN, Donahue SW, Wojda SJ, et al. The challenges of promoting osteogenesis in segmental bone defects and osteoporosis. Journal of orthopaedic research : official publication of the Orthopaedic Research Society 2018; 36(6): 1559-72.

166. Pelled G, Ben-Arav A, Hock C, et al. Direct gene therapy for bone regeneration: gene delivery, animal models, and outcome measures. Tissue engineering Part $B$, Reviews 2010; 16(1): 13-20.

167. Xing DG, Liu ZH, Gao HW, Ma WL, Nie L, Gong MZ. Effect of transplantation of marrow mesenchymal stem cells transfected with insulin-like growth factor-1 gene on fracture healing of rats with diabetes. Bratislavske lekarske listy 2015; 116(1): 64-8.

168. Nauth A, Miclau T, 3rd, Li R, Schemitsch EH. Gene therapy for fracture healing. Journal of orthopaedic trauma 2010; 24 Suppl 1: S17-24.

169. Evans CH. Gene delivery to bone. Advanced drug delivery reviews 2012; 64(12): 1331-40.

170. Chen Y, Luk KD, Cheung KM, et al. Gene therapy for new bone formation using adeno-associated viral bone morphogenetic protein-2 vectors. Gene therapy 2003; 10(16): 1345-53.

171. Tian K, Qi M, Wang L, et al. Two-stage therapeutic utility of ectopically formed bone tissue in skeletal muscle induced by adeno-associated virus containing bone morphogenetic protein-4 gene. Journal of orthopaedic surgery and research 2015; 10: 86.

172. Yang M, Ma QJ, Dang GT, Ma KT, Chen P, Zhou CY. Adeno-associated virus-mediated bone morphogenetic protein-7 gene transfer induces C2C12 cell differentiation into osteoblast lineage cells. Acta pharmacologica Sinica 2005; 26(8): 963-8.

173.T. T. Local inflammatory changes induced by fractures and soft tissue injuries. In: Pape HC, Sanders R, Borrelli Jr. J, eds. The poly-traumatized patient with fractures - a multi-disciplinary approach-. Berlin Heidelberg: Springer-Verlag; 2011: 19-31.

174. Schmidt-Bleek K, Schell H, Schulz N, et al. Inflammatory phase of bone healing initiates the regenerative healing cascade. Cell and tissue research 2012; 347(3): 567-73.

175. Lerner UH. Stimulation of bone resorption by the kallikrein-kinin system and the coagulation cascade. Acta orthopaedica Scandinavica Supplementum 1995; 266: $45-50$.

176. Sato N, Ichikawa J, Wako M, et al. Thrombin induced by the extrinsic pathway and PAR-1 regulated inflammation at the site of fracture repair. Bone 2016; 83: 23-34.

177. Vi L, Baht GS, Whetstone $\mathrm{H}$, et al. Macrophages promote osteoblastic differentiation in-vivo: implications in fracture repair and bone homeostasis. Journal of bone and mineral research : the official journal of the American Society for Bone and Mineral Research 2015; 30(6): 1090-102.
178. Vasconcelos DM, Goncalves RM, Almeida CR, et al. Fibrinogen scaffolds with immunomodulatory properties promote in vivo bone regeneration. Biomaterials 2016; 111: 163-78.

179. Santos SG, Lamghari M, Almeida CR, et al. Adsorbed fibrinogen leads to improved bone regeneration and correlates with differences in the systemic immune response. Acta biomaterialia 2013; 9(7): 7209-17.

180. Shiose S, Hata Y, Noda Y, et al. Fibrinogen stimulates in vitro angiogenesis by choroidal endothelial cells via autocrine VEGF. Graefe's archive for clinical and experimental ophthalmology = Albrecht von Graefes Archiv fur klinische und experimentelle Ophthalmologie 2004; 242(9): 777-83.

181. Yuasa M, Mignemi NA, Nyman JS, et al. Fibrinolysis is essential for fracture repair and prevention of heterotopic ossification. The Journal of clinical investigation 2015; 125(8): 3117-31.

182. Aeschlimann D, Mosher D, Paulsson M. Tissue transglutaminase and factor XIII in cartilage and bone remodeling. Seminars in thrombosis and hemostasis 1996; 22(5): 437-43.

183. Shi DY, Wang SJ. Advances of Coagulation Factor XIII. Chinese medical journal 2017; 130(2): 219-23.

184. Ploplis VA, French EL, Carmeliet P, Collen D, Plow EF. Plasminogen deficiency differentially affects recruitment of inflammatory cell populations in mice. Blood 1998; 91(6): 2005-9.

185. Khalil N, Corne S, Whitman C, Yacyshyn H. Plasmin regulates the activation of cell-associated latent TGFbeta 1 secreted by rat alveolar macrophages after in vivo bleomycin injury. American journal of respiratory cell and molecular biology 1996; 15(2): 252-9.

186. Yee JA, Yan L, Dominguez JC, Allan EH, Martin TJ. Plasminogen-dependent activation of latent transforming growth factor beta (TGF beta) by growing cultures of osteoblast-like cells. Journal of cellular physiology 1993; 157(3): 528-34.

187. Kawao N, Tamura Y, Okumoto K, et al. Plasminogen plays a crucial role in bone repair. Journal of bone and mineral research : the official journal of the American Society for Bone and Mineral Research 2013; 28(7): 1561-74.

188. Roth D, Piekarek M, Paulsson M, et al. Plasmin modulates vascular endothelial growth factor-Amediated angiogenesis during wound repair. The American journal of pathology 2006; 168(2): 670-84.

189. Bravo D, Josephson AM, Bradaschia-Correa V, et al. Temporary inhibition of the plasminogen activator inhibits periosteal chondrogenesis and promotes periosteal osteogenesis during appendicular bone fracture healing. Bone 2018; 112: 97-106.

190. Schoenecker J, Mignemi N, Stutz C, et al. 2010 Young Investigator Award winner: Therapeutic aprotinin stimulates osteoblast proliferation but inhibits differentiation and bone matrix mineralization. Spine 2010; 35(9): 1008-16.

191. Fukumoto S, Allan EH, Yee JA, Gelehrter TD, Martin TJ. Plasminogen activator regulation in osteoblasts: parathyroid hormone inhibition of type-1 plasminogen activator inhibitor and its mRNA. Journal of cellular physiology 1992; 152(2): 346-55. 
192. Hamilton JA, Lingelbach S, Partridge NC, Martin TJ. Regulation of plasminogen activator production by bone-resorbing hormones in normal and malignant osteoblasts. Endocrinology 1985; 116(6): 2186-91.

193. Daci E, Udagawa N, Martin TJ, Bouillon R, Carmeliet G. The role of the plasminogen system in bone resorption in vitro. Journal of bone and mineral research : the official journal of the American Society for Bone and Mineral Research 1999; 14(6): 946-52.

194. Everts V, Daci E, Tigchelaar-Gutter W, et al. Plasminogen activators are involved in the degradation of bone by osteoclasts. Bone 2008; 43(5): 915-20.

195. Ehrnthaller C, Huber-Lang M, Kovtun A, et al. C5aR inhibition in the early inflammatory phase does not affect bone regeneration in a model of uneventful fracture healing. European journal of medical research 2016; 21(1): 42.

196. Huber-Lang M, Younkin EM, Sarma JV, et al. Generation of $\mathrm{C} 5 \mathrm{a}$ by phagocytic cells. The American journal of pathology 2002; 161(5): 1849-59.

197. Wiggins RC, Giclas PC, Henson PM. Chemotactic activity generated from the fifth component of complement by plasma kallikrein of the rabbit. The Journal of experimental medicine 1981; 153(6): 1391404.

198. Tsukamoto T. Local inflammatory changes induced by fractures and soft tissue injuries. In: Pape HC SR, Borrelli Jr. J, ed. The poly-traumatized patient with fractures - a multi-disciplinary approach-. Berlin Heidelberg: Springer-Verlag; 2011: 19-31.

199. Rutkowski MJ, Sughrue ME, Kane AJ, Ahn BJ, Fang S, Parsa AT. The complement cascade as a mediator of tissue growth and regeneration. Inflammation research: official journal of the European Histamine Research Society [et al] 2010; 59(11): 897-905.

200. Ignatius A, Schoengraf P, Kreja L, et al. Complement $\mathrm{C} 3 \mathrm{a}$ and $\mathrm{C5a}$ modulate osteoclast formation and inflammatory response of osteoblasts in synergism with IL-1beta. Journal of cellular biochemistry 2011; 112(9): 2594-605.

201. Modinger Y, Rapp AE, Vikman A, et al. Reduced Terminal Complement Complex Formation in Mice Manifests in Low Bone Mass and Impaired Fracture Healing. The American journal of pathology 2019; 189(1): 147-61.

202. Huber-Lang M, Kovtun A, Ignatius A. The role of complement in trauma and fracture healing. Seminars in immunology 2013; 25(1): 73-8.

203. Bergdolt S, Kovtun A, Hagele Y, et al. Osteoblastspecific overexpression of complement receptor C5aR1 impairs fracture healing. PloS one 2017; 12(6): $\mathrm{e} 0179512$.

204. Ehrnthaller C, Huber-Lang M, Nilsson P, et al. Complement C3 and C5 deficiency affects fracture healing. PloS one 2013; 8(11): e81341.

205. Van der Ende J, Van Baardewijk LJ, Sier CF, Schipper IB. Bone healing and mannose-binding lectin. International journal of surgery 2013; 11(4): 296-300.

206. Bernhold Brechter A. Kinins: important regulators in inflammation induced bone resorption. Umeå, Sweden: Umeå University; 2006.

207. Brechter AB, Persson E, Lundgren I, Lerner UH. Kinin B1 and B2 receptor expression in osteoblasts and fibroblasts is enhanced by interleukin-1 and tumour necrosis factor-alpha. Effects dependent on activation of NF-kappaB and MAP kinases. Bone 2008; 43(1): 72-83.

208. Zhang Y, Wang L, Song Y, Zhao X, Wong MS, Zhang W. Renin inhibitor aliskiren exerts beneficial effect on trabecular bone by regulating skeletal renin-angiotensin system and kallikrein-kinin system in ovariectomized mice. Osteoporosis international : a journal established as result of cooperation between the European Foundation for Osteoporosis and the National Osteoporosis Foundation of the USA 2016; 27(3): 1083-92.

209. Gibon E, Lu LY, Nathan K, Goodman SB. Inflammation, ageing, and bone regeneration. Journal of orthopaedic translation 2017; 10: 28-35.

210. Dinarello CA. Proinflammatory cytokines. Chest 2000; 118(2): 503-8.

211. Chan JK, Glass GE, Ersek A, et al. Low-dose TNF augments fracture healing in normal and osteoporotic bone by up-regulating the innate immune response. EMBO molecular medicine 2015; 7(5): 547-61.

212. Neagoe PE, Brkovic A, Hajjar F, Sirois MG. Expression and release of angiopoietin-1 from human neutrophils: intracellular mechanisms. Growth factors 2009; 27(6): 335-44.

213. Gerstenfeld LC, Cho TJ, Kon T, et al. Impaired fracture healing in the absence of TNF-alpha signaling: the role of TNF-alpha in endochondral cartilage resorption. Journal of bone and mineral research : the official journal of the American Society for Bone and Mineral Research 2003; 18(9): 1584-92.

214. Kayal RA, Siqueira M, Alblowi J, et al. TNF-alpha mediates diabetes-enhanced chondrocyte apoptosis during fracture healing and stimulates chondrocyte apoptosis through FOX01. Journal of bone and mineral research : the official journal of the American Society for Bone and Mineral Research 2010; 25(7): 1604-15.

215. Sonomoto K, Yamaoka K, Oshita K, et al. Interleukin1beta induces differentiation of human mesenchymal stem cells into osteoblasts via the Wnt-5a/receptor tyrosine kinase-like orphan receptor 2 pathway. Arthritis and rheumatism 2012; 64(10): 3355-63.

216. Gilbert LC, Rubin J, Nanes MS. The p55 TNF receptor mediates TNF inhibition of osteoblast differentiation independently of apoptosis. American journal of physiology Endocrinology and metabolism 2005; 288(5): E1011-8.

217. Lacey DC, Simmons PJ, Graves SE, Hamilton JA. Proinflammatory cytokines inhibit osteogenic differentiation from stem cells: implications for bone repair during inflammation. Osteoarthritis and cartilage 2009; 17(6): 735-42.

218. Sang C, Zhang Y, Chen F, et al. Tumor necrosis factor alpha suppresses osteogenic differentiation of MSCs by inhibiting semaphorin $3 \mathrm{~B}$ via Wnt/beta-catenin signaling in estrogen-deficiency induced osteoporosis. Bone 2016; 84: 78-87.

219. Osta B, Benedetti G, Miossec P. Classical and Paradoxical Effects of TNF-alpha on Bone Homeostasis. Frontiers in immunology 2014; 5: 48.

220. Glass GE, Chan JK, Freidin A, Feldmann M, Horwood NJ, Nanchahal J. TNF-alpha promotes fracture repair by augmenting the recruitment and differentiation of muscle-derived stromal cells. Proceedings of the 
National Academy of Sciences of the United States of America 2011; 108(4): 1585-90.

221. Hess K, Ushmorov A, Fiedler J, Brenner RE, Wirth T. TNFalpha promotes osteogenic differentiation of human mesenchymal stem cells by triggering the NF-kappaB signaling pathway. Bone 2009; 45(2): 367-76.

222. Lee YM, Fujikado N, Manaka H, Yasuda H, Iwakura Y. IL-1 plays an important role in the bone metabolism under physiological conditions. International immunology 2010; 22(10): 805-16.

223. Murakami S, Lefebvre V, de Crombrugghe B. Potent inhibition of the master chondrogenic factor Sox 9 gene by interleukin- 1 and tumor necrosis factor-alpha. The Journal of biological chemistry 2000; 275(5): 3687-92.

224. Moverare-Skrtic S, Henning P, Liu X, et al. Osteoblast-derived WNT16 represses osteoclastogenesis and prevents cortical bone fragility fractures. Nature medicine 2014; 20(11): 1279-88.

225. Garbers C, Rose-John S. Dissecting Interleukin-6 Classic- and Trans-Signaling in Inflammation and Cancer. Methods in molecular biology 2018; 1725: 127 40.

226. Franchimont N, Wertz S, Malaise M. Interleukin-6: An osteotropic factor influencing bone formation? Bone 2005; 37(5): 601-6.

227. Kondo M, Yamaoka K, Sakata K, et al. Contribution of the Interleukin-6/STAT-3 Signaling Pathway to Chondrogenic Differentiation of Human Mesenchymal Stem Cells. Arthritis \& rheumatology 2015; 67(5): 125060.

228. Blanchard F, Duplomb L, Baud'huin M, Brounais B. The dual role of IL-6-type cytokines on bone remodeling and bone tumors. Cytokine \& growth factor reviews 2009; 20(1): 19-28.

229. Thomas MV, Puleo DA. Infection, inflammation, and bone regeneration: a paradoxical relationship. Journal of dental research 2011; 90(9): 1052-61.

230. Cho TJ, Kim JA, Chung CY, et al. Expression and role of interleukin-6 in distraction osteogenesis. Calcified tissue international 2007; 80(3): 192-200.

231. Wong PK, Quinn JM, Sims NA, van Nieuwenhuijze A, Campbell IK, Wicks IP. Interleukin-6 modulates production of $\mathrm{T}$ lymphocyte-derived cytokines in antigen-induced arthritis and drives inflammationinduced osteoclastogenesis. Arthritis and rheumatism 2006; 54(1): 158-68.

232. Kudo 0, Sabokbar A, Pocock A, Itonaga I, Fujikawa Y, Athanasou NA. Interleukin-6 and interleukin-11 support human osteoclast formation by a RANKLindependent mechanism. Bone 2003; 32(1): 1-7.

233. Huang L, Liu S, Song T, Zhang W, Fan J, Liu Y. Blockade of Interleukin 6 by Rat Anti-mouse Interleukin 6 Receptor Antibody Promotes Fracture Healing. Biochemistry Biokhimiia 2017; 82(10): 1193-9.

234. Prystaz K, Kaiser K, Kovtun A, et al. Distinct Effects of IL-6 Classic and Trans-Signaling in Bone Fracture Healing. The American journal of pathology 2018; 188(2): 474-90.

235. Ono T, Takayanagi H. Osteoimmunology in Bone Fracture Healing. Current osteoporosis reports 2017; 15(4): 367-75.
236. Korn T, Bettelli E, Oukka M, Kuchroo VK. IL-17 and Th17 Cells. Annual review of immunology 2009; 27: 485517.

237. Kitami S, Tanaka H, Kawato T, et al. IL-17A suppresses the expression of bone resorption-related proteinases and osteoclast differentiation via IL-17RA or IL-17RC receptors in RAW264.7 cells. Biochimie 2010; 92(4): 398-404.

238. Croes M, Oner FC, van Neerven D, et al. Proinflammatory T cells and IL-17 stimulate osteoblast differentiation. Bone 2016; 84: 262-70.

239. Ono T, Okamoto K, Nakashima T, et al. IL-17producing gammadelta $\mathrm{T}$ cells enhance bone regeneration. Nature communications 2016; 7: 10928.

240. Kim YG, Park JW, Lee JM, et al. IL-17 inhibits osteoblast differentiation and bone regeneration in rat. Archives of oral biology 2014; 59(9): 897-905.

241. Shaw AT, Maeda Y, Gravallese EM. IL-17A deficiency promotes periosteal bone formation in a model of inflammatory arthritis. Arthritis research \& therapy 2016; 18(1): 104.

242. Nam D, Mau E, Wang Y, et al. T-lymphocytes enable osteoblast maturation via IL-17F during the early phase of fracture repair. PloS one 2012; 7(6): e40044.

243. Silfversward CJ, Larsson S, Ohlsson C, Frost A, Nilsson 0. Reduced cortical bone mass in mice with inactivation of interleukin-4 and interleukin-13. Journal of orthopaedic research : official publication of the Orthopaedic Research Society 2007; 25(6): 725-31.

244. Wang Y, Wu NN, Mou YQ, Chen L, Deng ZL. Inhibitory effects of recombinant IL-4 and recombinant IL-13 on UHMWPE-induced bone destruction in the murine air pouch model. The Journal of surgical research 2013; 180(2): e73-81.

245. Loi F, Cordova LA, Zhang R, et al. The effects of immunomodulation by macrophage subsets on osteogenesis in vitro. Stem cell research \& therapy 2016; 7: 15.

246. G G. New insights into the molecular mechanism of interleukin-10-mediated immunosuppression. J Leukoc Biol 2005; 77(1): 3-15.

247. Dresner-Pollak R, Gelb N, Rachmilewitz D, Karmeli $\mathrm{F}$, Weinreb M. Interleukin 10-deficient mice develop osteopenia, decreased bone formation, and mechanical fragility of long bones. Gastroenterology 2004; 127(3): 792-801.

248. Kimura D, Miyakoda M, Kimura K, et al. Interleukin27-Producing CD4(+) T Cells Regulate Protective Immunity during Malaria Parasite Infection. Immunity 2016; 44(3): 672-82.

249. Figueiredo Neto M, Figueiredo ML. Combination of Interleukin-27 and MicroRNA for Enhancing Expression of Anti-Inflammatory and Proosteogenic Genes. Arthritis 2017; 2017: 6365857.

250. Xu X, Zheng L, Yuan Q, et al. Transforming growth factor-beta in stem cells and tissue homeostasis. Bone research 2018; 6: 2.

251. Hering S, Isken E, Knabbe C, et al. TGFbeta1 and TGFbeta2 mRNA and protein expression in human bone samples. Experimental and clinical endocrinology \& diabetes : official journal, German Society of Endocrinology [and] German Diabetes Association 2001; 109(4): 217-26. 
252. Fisher DM, Wong JM, Crowley C, Khan WS. Preclinical and clinical studies on the use of growth factors for bone repair: a systematic review. Current stem cell research \& therapy 2013; 8(3): 260-8.

253. Tsiridis E, Upadhyay N, Giannoudis P. Molecular aspects of fracture healing: which are the important molecules? Injury 2007; 38 Suppl 1: S11-25.

254. Zhao L, Jiang S, Hantash BM. Transforming growth factor beta1 induces osteogenic differentiation of murine bone marrow stromal cells. Tissue engineering Part A 2010; 16(2): 725-33.

255. Wan M, Li C, Zhen G, et al. Injury-activated transforming growth factor beta controls mobilization of mesenchymal stem cells for tissue remodeling. Stem cells 2012; 30(11): 2498-511.

256. Zimmermann G, Muller U, Wentzensen A. The value of laboratory and imaging studies in the evaluation of long-bone non-unions. Injury 2007; 38 Suppl 2: S33-7.

257. Papanagiotou M, Dailiana ZH, Karachalios T, et al. RhBMP-7 for the treatment of nonunion of fractures of long bones. The bone \& joint journal 2015; 97-B(7): 9971003.

258. Brannan PS, Gaston RG, Loeffler BJ, Lewis DR. Complications With the Use of BMP-2 in Scaphoid Nonunion Surgery. The Journal of hand surgery 2016; 41(5): 602-8.

259. Garrison KR, Donell S, Ryder J, et al. Clinical effectiveness and cost-effectiveness of bone morphogenetic proteins in the non-healing of fractures and spinal fusion: a systematic review. Health technology assessment 2007; 11(30): 1-150, iii-iv.

260. Daluiski A, Ramsey KE, Shi Y, et al. Cyclooxygenase2 inhibitors in human skeletal fracture healing. Orthopedics 2006; 29(3): 259-61.

261. Muinos-Lopez E, Ripalda-Cemborain P, LopezMartinez T, et al. Hypoxia and Reactive Oxygen Species Homeostasis in Mesenchymal Progenitor Cells Define a Molecular Mechanism for Fracture Nonunion. Stem cells 2016; 34(9): 2342-53.

262. Matthews SJ. Biological activity of bone morphogenetic proteins (BMP's). Injury 2005; 36 Suppl 3: S34-7.

263. Giannoudis PV. Fracture healing and bone regeneration: autologous bone grafting or BMPs? Injury 2009; 40(12): 1243-4.

264. Moghaddam A, Breier L, Haubruck P, et al. Nonunions treated with bone morphogenic protein 7 : introducing the quantitative measurement of human serum cytokine levels as promising tool in evaluation of adjunct non-union therapy. Journal of inflammation 2016; 13: 3.

265. Haubruck P, Kammerer A, Korff S, et al. The treatment of nonunions with application of BMP-7 increases the expression pattern for angiogenic and inflammable cytokines: a matched pair analysis. Journal of inflammation research 2016; 9: 155-65.

266. Garrison KR, Shemilt I, Donell S, et al. Bone morphogenetic protein (BMP) for fracture healing in adults. The Cochrane database of systematic reviews 2010; (6): CD006950.

267. Schmidmaier G, Moghaddam A. [Long Bone Nonunion]. Zeitschrift fur Orthopadie und Unfallchirurgie 2015; 153(6): 659-74; quiz 75-6.
268. Giannoudis PV, Kanakaris NK, Dimitriou R, Gill I, Kolimarala V, Montgomery RJ. The synergistic effect of autograft and BMP-7 in the treatment of atrophic nonunions. Clinical orthopaedics and related research 2009; 467(12): 3239-48.

269. Krishnakumar GS, Roffi A, Reale D, Kon E, Filardo G. Clinical application of bone morphogenetic proteins for bone healing: a systematic review. International orthopaedics 2017; 41(6): 1073-83.

270. Tsuji K, Bandyopadhyay A, Harfe BD, et al. BMP2 activity, although dispensable for bone formation, is required for the initiation of fracture healing. Nature genetics 2006; 38(12): 1424-9.

271. Westhauser F, Zimmermann G, Moghaddam S, et al. Reaming in treatment of non-unions in long bones: cytokine expression course as a tool for evaluation of non-union therapy. Archives of orthopaedic and trauma surgery 2015; 135(8): 1107-16.

272. Glienke J, Schmitt AO, Pilarsky C, et al. Differential gene expression by endothelial cells in distinct angiogenic states. European journal of biochemistry / FEBS 2000; 267(9): 2820-30.

273. Takemoto R, Forman J, Taormina DP, Egol KA. No advantage to rhBMP-2 in addition to autogenous graft for fracture nonunion. Orthopedics 2014; 37(6): e52530.

274. von Ruden C, Morgenstern M, Hierholzer C, et al. The missing effect of human recombinant Bone Morphogenetic Proteins BMP-2 and BMP-7 in surgical treatment of aseptic forearm nonunion. Injury 2016; 47(4): 919-24.

275. Sebald HJ, Klenke FM, Siegrist M, Albers CE, Sebald W, Hofstetter W. Inhibition of endogenous antagonists with an engineered BMP-2 variant increases BMP-2 efficacy in rat femoral defect healing. Acta biomaterialia 2012; 8(10): 3816-20.

276. Bikle D, Majumdar S, Laib A, et al. The skeletal structure of insulin-like growth factor I-deficient mice. Journal of bone and mineral research : the official journal of the American Society for Bone and Mineral Research 2001; 16(12): 2320-9.

277. He J, Rosen CJ, Adams DJ, Kream BE. Postnatal growth and bone mass in mice with IGF-I haploinsufficiency. Bone 2006; 38(6): 826-35.

278. Wang T, Wang Y, Menendez A, et al. OsteoblastSpecific Loss of IGF1R Signaling Results in Impaired Endochondral Bone Formation During Fracture Healing. Journal of bone and mineral research : the official journal of the American Society for Bone and Mineral Research 2015; 30(9): 1572-84.

279. Raschke M, Wildemann B, Inden $P$, et al. Insulin-like growth factor-1 and transforming growth factor-beta1 accelerates osteotomy healing using polylactide-coated implants as a delivery system: a biomechanical and histological study in minipigs. Bone 2002; 30(1): 14451.

280. Mathieu M, Rigutto S, Ingels A, et al. Decreased pool of mesenchymal stem cells is associated with altered chemokines serum levels in atrophic nonunion fractures. Bone 2013; 53(2): 391-8.

281. Phillips AM. Overview of the fracture healing cascade. Injury 2005; 36 Suppl 3: S5-7. 
282. Weiss S, Henle P, Bidlingmaier M, Moghaddam A, Kasten P, Zimmermann G. Systemic response of the $\mathrm{GH} / \mathrm{IGF}-\mathrm{I}$ axis in timely versus delayed fracture healing. Growth hormone \& IGF research: official journal of the Growth Hormone Research Society and the International IGF Research Society 2008; 18(3): 205-12.

283. Fischer C, Doll J, Tanner M, et al. Quantification of TGF-ss1, PDGF and IGF-1 cytokine expression after fracture treatment vs. non-union therapy via masquelet. Injury 2016; 47(2): 342-9.

284. Sheng MH, Lau KH, Baylink DJ. Role of Osteocytederived Insulin-Like Growth Factor I in Developmental Growth, Modeling, Remodeling, and Regeneration of the Bone. Journal of bone metabolism 2014; 21(1): 41-54.

285. Lau KW, Rundle CH, Zhou XD, Baylink DJ, Sheng MH. Conditional deletion of IGF-I in osteocytes unexpectedly accelerates bony union of the fracture gap in mice. Bone 2016; 92: 18-28.

286. Koh A, Niikura T, Lee SY, et al. Differential gene expression and immunolocalization of insulin-like growth factors and insulin-like growth factor binding proteins between experimental nonunions and standard healing fractures. Journal of orthopaedic research : official publication of the Orthopaedic Research Society 2011; 29(12): 1820-6.

287. Kosaki N, Takaishi H, Kamekura S, et al. Impaired bone fracture healing in matrix metalloproteinase-13 deficient mice. Biochemical and biophysical research communications 2007; 354(4): 846-51.

288. Colnot C, Thompson Z, Miclau T, Werb Z, Helms JA. Altered fracture repair in the absence of MMP9. Development 2003; 130(17): 4123-33.

289. Wigner NA, Kulkarni N, Yakavonis M, et al. Urine matrix metalloproteinases (MMPs) as biomarkers for the progression of fracture healing. Injury 2012; 43(3): 274-8.

290. Zimna A, Kurpisz M. Hypoxia-Inducible Factor-1 in Physiological and Pathophysiological Angiogenesis: Applications and Therapies. BioMed research international 2015; 2015: 549412.

291. Rankin EB, Wu C, Khatri R, et al. The HIF signaling pathway in osteoblasts directly modulates erythropoiesis through the production of EPO. Cell 2012; 149(1): 63-74.

292. Schipani E, Maes C, Carmeliet G, Semenza GL. Regulation of osteogenesis-angiogenesis coupling by HIFs and VEGF. Journal of bone and mineral research : the official journal of the American Society for Bone and Mineral Research 2009; 24(8): 1347-53.

293. Fan L, Li J, Yu Z, Dang X, Wang K. The hypoxiainducible factor pathway, prolyl hydroxylase domain protein inhibitors, and their roles in bone repair and regeneration. BioMed research international 2014; 2014: 239356.

294. Jiang BH, Semenza GL, Bauer C, Marti HH. Hypoxiainducible factor 1 levels vary exponentially over a physiologically relevant range of 02 tension. The American journal of physiology 1996; 271(4 Pt 1): C1172-80.

295. Semenza GL. HIF-1 and mechanisms of hypoxia sensing. Current opinion in cell biology 2001; 13(2): 16771.
296. Hung SC, Pochampally RR, Hsu SC, et al. Short-term exposure of multipotent stromal cells to low oxygen increases their expression of CX3CR1 and CXCR4 and their engraftment in vivo. PloS one 2007; 2(5): e416.

297. Tsai CC, Yew TL, Yang DC, Huang WH, Hung SC. Benefits of hypoxic culture on bone marrow multipotent stromal cells. American journal of blood research 2012; 2(3): 148-59.

298. Ferrara N, Gerber HP, LeCouter J. The biology of VEGF and its receptors. Nature medicine 2003; 9(6): 669-76.

299. Beamer B, Hettrich C, Lane J. Vascular endothelial growth factor: an essential component of angiogenesis and fracture healing. HSS journal : the musculoskeletal journal of Hospital for Special Surgery 2010; 6(1): 85-94.

300. Ai-Aql ZS, Alagl AS, Graves DT, Gerstenfeld LC, Einhorn TA. Molecular mechanisms controlling bone formation during fracture healing and distraction osteogenesis. Journal of dental research 2008; 87(2): 107-18.

301. Street J, Winter D, Wang JH, Wakai A, McGuinness A, Redmond HP. Is human fracture hematoma inherently angiogenic? Clinical orthopaedics and related research 2000; (378): 224-37.

302. Street JT, Wang JH, Wu QD, Wakai A, McGuinness A, Redmond HP. The angiogenic response to skeletal injury is preserved in the elderly. Journal of orthopaedic research : official publication of the Orthopaedic Research Society 2001; 19(6): 1057-66.

303. Carlevaro MF, Cermelli S, Cancedda R, Descalzi Cancedda F. Vascular endothelial growth factor (VEGF) in cartilage neovascularization and chondrocyte differentiation: auto-paracrine role during endochondral bone formation. Journal of cell science 2000; 113 ( Pt 1): 59-69.

304. Testa U, Pannitteri G, Condorelli GL. Vascular endothelial growth factors in cardiovascular medicine. Journal of cardiovascular medicine 2008; 9(12): 1190221.

305. Ozturk BY, Inci I, Egri S, et al. The treatment of segmental bone defects in rabbit tibiae with vascular endothelial growth factor (VEGF)-loaded gelatin/hydroxyapatite "cryogel" scaffold. European journal of orthopaedic surgery \& traumatology : orthopedie traumatologie 2013; 23(7): 767-74.

306. Weiss S, Zimmermann G, Pufe T, Varoga D, Henle P. The systemic angiogenic response during bone healing. Archives of orthopaedic and trauma surgery 2009; 129(7): 989-97.

307. Tzeng DY, Deuel TF, Huang JS, Senior RM, Boxer LA, Baehner RL. Platelet-derived growth factor promotes polymorphonuclear leukocyte activation. Blood 1984; 64(5): 1123-8.

308. Andrew JG, Hoyland JA, Freemont AJ, Marsh DR. Platelet-derived growth factor expression in normally healing human fractures. Bone 1995; 16(4): 455-60.

309. Schwabe P, Simon P, Kronbach Z, Schmidmaier G, Wildemann B. A pilot study investigating the histology and growth factor content of human non-union tissue. International orthopaedics 2014; 38(12): 2623-9.

310. Griffith JW, Sokol CL, Luster AD. Chemokines and chemokine receptors: positioning cells for host defense 
and immunity. Annual review of immunology 2014; 32 : 659-702.

311. Xu C, Hasan SS, Schmidt I, et al. Arteries are formed by vein-derived endothelial tip cells. Nature communications 2014; 5: 5758.

312. Ishikawa $\mathrm{M}$, Ito $\mathrm{H}$, Kitaori $\mathrm{T}$, et al. MCP/CCR2 signaling is essential for recruitment of mesenchymal progenitor cells during the early phase of fracture healing. PloS one 2014; 9(8): e104954.

313. Xing Z, Lu C, Hu D, et al. Multiple roles for CCR2 during fracture healing. Disease models \& mechanisms 2010; 3(7-8): 451-8.

314. Herrero AB, Garcia-Gomez A, Garayoa M, et al. Effects of IL-8 Up-Regulation on Cell Survival and Osteoclastogenesis in Multiple Myeloma. The American journal of pathology 2016; 186(8): 2171-82.

315. Bastidas-Coral AP, Bakker AD, Zandieh-Doulabi B, et al. Cytokines TNF-alpha, IL-6, IL-17F, and IL-4 Differentially Affect Osteogenic Differentiation of Human Adipose Stem Cells. Stem cells international 2016; 2016: 1318256

316. Brat DJ, Bellail AC, Van Meir EG. The role of interleukin-8 and its receptors in gliomagenesis and tumoral angiogenesis. Neuro-oncology 2005; 7(2): 12233.

317. Kawakami Y, Ii M, Matsumoto T, et al. SDF-1/CXCR4 axis in Tie2-lineage cells including endothelial progenitor cells contributes to bone fracture healing. Journal of bone and mineral research : the official journal of the American Society for Bone and Mineral Research 2015; 30(1): 95-105.

318. Toupadakis CA, Wong A, Genetos DC, et al. Longterm administration of AMD3100, an antagonist of SDF1/CXCR4 signaling, alters fracture repair. Journal of orthopaedic research : official publication of the Orthopaedic Research Society 2012; 30(11): 1853-9.

319. Dennis EA, Norris PC. Eicosanoid storm in infection and inflammation. Nature reviews Immunology 2015; 15(8): 511-23.

320. Xie C, Liang B, Xue M, et al. Rescue of impaired fracture healing in COX-2-/- mice via activation of prostaglandin E2 receptor subtype 4 . The American journal of pathology 2009; 175(2): 772-85.

321. Tanaka M, Sakai A, Uchida S, et al. Prostaglandin E2 receptor (EP4) selective agonist (ONO-4819.CD) accelerates bone repair of femoral cortex after drill-hole injury associated with local upregulation of bone turnover in mature rats. Bone 2004; 34(6): 940-8.

322. Wijnands KA, Brink PR, Weijers PH, Dejong $\mathrm{CH}$, Poeze M. Impaired fracture healing associated with amino acid disturbances. The American journal of clinical nutrition 2012; 95(5): 1270-7.

323. Diwan AD, Wang MX, Jang D, Zhu W, Murrell GA. Nitric oxide modulates fracture healing. Journal of bone and mineral research : the official journal of the American Society for Bone and Mineral Research 2000; 15(2): 34251.

324. Luiking YC, Poeze M, Ramsay G, Deutz NE. The role of arginine in infection and sepsis. JPEN Journal of parenteral and enteral nutrition 2005; 29(1 Suppl): S704.

325. Haltmeier T, Inaba K, Schnuriger B, et al. Factors affecting the caloric and protein intake over time in critically ill trauma patients. The Journal of surgical research 2018; 226: 64-71.

326. Rogobete AF, Sandesc D, Papurica M, et al. The influence of metabolic imbalances and oxidative stress on the outcome of critically ill polytrauma patients: a review. Burns \& trauma 2017; 5: 8.

327. Baht GS, Vi L, Alman BA. The Role of the Immune Cells in Fracture Healing. Current osteoporosis reports 2018; 16(2): 138-45.

328. Kusumbe AP, Ramasamy SK, Adams RH. Coupling of angiogenesis and osteogenesis by a specific vessel subtype in bone. Nature 2014; 507(7492): 323-8.

329. Murnaghan M, Li G, Marsh DR. Nonsteroidal antiinflammatory drug-induced fracture nonunion: an inhibition of angiogenesis? The Journal of bone and joint surgery American volume 2006; 88 Suppl 3: 140-7.

330. Tomlinson RE, McKenzie JA, Schmieder AH, Wohl GR, Lanza GM, Silva MJ. Angiogenesis is required for stress fracture healing in rats. Bone 2013; 52(1): 212-9.

331. Rajfer RA, Kilic A, Neviaser AS, et al. Enhancement of fracture healing in the rat, modulated by compounds that stimulate inducible nitric oxide synthase: Acceleration of fracture healing via inducible nitric oxide synthase. Bone \& joint research 2017; 6(2): 90-7.

332. Baldik Y, Talu U, Altinel L, Bilge H, Demiryont M, Aykac-Toker G. Bone healing regulated by nitric oxide: an experimental study in rats. Clinical orthopaedics and related research 2002; (404): 343-52.

333. Corbett SA, Hukkanen M, Batten J, McCarthy ID, Polak JM, Hughes SP. Nitric oxide in fracture repair. Differential localisation, expression and activity of nitric oxide synthases. The Journal of bone and joint surgery British volume 1999; 81(3): 531-7.

334. Zhu W, Diwan AD, Lin JH, Murrell GA. Nitric oxide synthase isoforms during fracture healing. Journal of bone and mineral research : the official journal of the American Society for Bone and Mineral Research 2001; 16(3): 535-40.

335. Tomlinson RE, Shoghi KI, Silva MJ. Nitric oxidemediated vasodilation increases blood flow during the early stages of stress fracture healing. Journal of applied physiology 2014; 116(4): 416-24.

336. Lundberg JO, Weitzberg E. NO generation from inorganic nitrate and nitrite: Role in physiology, nutrition and therapeutics. Archives of pharmacal research 2009; 32(8): 1119-26.

337. Stamler JS, Meissner G. Physiology of nitric oxide in skeletal muscle. Physiological reviews 2001; 81(1): 20937.

338. Lundberg JO, Weitzberg E, Gladwin MT. The nitratenitrite-nitric oxide pathway in physiology and therapeutics. Nature reviews Drug discovery 2008; 7(2): 156-67.

339. Curis E, Nicolis I, Moinard C, et al. Almost all about citrulline in mammals. Amino acids 2005; 29(3): 177 205.

340. Meesters DM, Neubert S, Wijnands KAP, et al. Deficiency of inducible and endothelial nitric oxide synthase results in diminished bone formation and delayed union and nonunion development. Bone 2016; 83: 111-8.

341. Corbett SA, McCarthy ID, Batten J, Hukkanen M, Polak JM, Hughes SP. Nitric oxide mediated 
vasoreactivity during fracture repair. Clinical orthopaedics and related research 1999; (365): 247-53.

342. Luiking YC, Poeze M, Ramsay G, Deutz NE. Reduced citrulline production in sepsis is related to diminished de novo arginine and nitric oxide production. The American journal of clinical nutrition 2009; 89(1): 14252.

343. Lee RH, Efron D, Tantry U, Barbul A. Nitric oxide in the healing wound: a time-course study. The Journal of surgical research 2001; 101(1): 104-8.

344. Kdolsky RK, Mohr W, Savidis-Dacho H, et al. The influence of oral L-arginine on fracture healing: an animal study. Wiener klinische Wochenschrift 2005; 117(19-20): 693-701.

345. Xia W, Szomor Z, Wang Y, Murrell GA. Nitric oxide enhances collagen synthesis in cultured human tendon cells. Journal of orthopaedic research : official publication of the Orthopaedic Research Society 2006; 24(2): 159-72.

346. Wijnands KA, Castermans TM, Hommen MP, Meesters DM, Poeze M. Arginine and citrulline and the immune response in sepsis. Nutrients 2015; 7(3): 142663.

347. Shen JQ, Yang QL, Xue Y, et al. Inducible nitric oxide synthase response and associated cytokine gene expression in the spleen of mice infected with Clonorchis sinensis. Parasitology research 2015; 114(5): 1661-70.

348. Melikian N, Seddon MD, Casadei B, Chowienczyk PJ, Shah AM. Neuronal nitric oxide synthase and human vascular regulation. Trends in cardiovascular medicine 2009; 19(8): 256-62.

349. Castillo L, deRojas TC, Chapman TE, et al. Splanchnic metabolism of dietary arginine in relation to nitric oxide synthesis in normal adult man. Proceedings of the National Academy of Sciences of the United States of America 1993; 90(1): 193-7.

350. Wu G. Intestinal mucosal amino acid catabolism. The Journal of nutrition 1998; 128(8): 1249-52.

351. Morris SM, Jr. Regulation of enzymes of the urea cycle and arginine metabolism. Annual review of nutrition 2002; 22: 87-105.

352. van de Poll MC, Siroen MP, van Leeuwen PA, et al. Interorgan amino acid exchange in humans: consequences for arginine and citrulline metabolism. The American journal of clinical nutrition 2007; 85(1): 167-72.

353. Boger RH, Tsikas D, Bode-Boger SM, PhivthongNgam L, Schwedhelm E, Frolich JC. Hypercholesterolemia impairs basal nitric oxide synthase turnover rate: a study investigating the conversion of L-[guanidino-(15) N(2)]-arginine to (15)N-labeled nitrate by gas chromatography--mass spectrometry. Nitric oxide : biology and chemistry / official journal of the Nitric Oxide Society 2004; 11(1): 18.

354. Luiking YC, Poeze M, Dejong CH, Ramsay G, Deutz NE. Sepsis: an arginine deficiency state? Critical care medicine 2004; 32(10): 2135-45.

355. Yaman F, Acikan I, Dundar S, et al. Dietary arginine silicate inositol complex increased bone healing: histologic and histomorphometric study. Drug design, development and therapy 2016; 10: 2081-6.
356. Fearon WR. The carbamido diacetyl reaction: a test for citrulline. The Biochemical journal 1939; 33(6): 9027.

357. Haines RJ, Pendleton LC, Eichler DC. Argininosuccinate synthase: at the center of arginine metabolism. International journal of biochemistry and molecular biology 2011; 2(1): 8-23.

358. Osowska S, Duchemann T, Walrand S, et al. Citrulline modulates muscle protein metabolism in old malnourished rats. American journal of physiology Endocrinology and metabolism 2006; 291(3): E582-6.

359. Windmueller HG, Spaeth AE. Source and fate of circulating citrulline. The American journal of physiology 1981; 241(6): E473-80.

360. Wu G, MS MJ. Arginine in mammals. In: C. L, ed. Metabolic and Therapeutic Aspects of Amino acids in Clinical Nutrition. 2nd ed. Boca Raton, Fl; 2004: 153-67.

361. Wu G. Urea synthesis in enterocytes of developing pigs. The Biochemical journal 1995; 312 ( Pt 3): 717-23.

362. Morris SM, Jr. Enzymes of arginine metabolism. The Journal of nutrition 2004; 134(10 Suppl): 2743S-7S; discussion 65S-67S.

363. Osowska S, Moinard C, Neveux N, Loi C, Cynober L. Citrulline increases arginine pools and restores nitrogen balance after massive intestinal resection. Gut 2004; 53(12): 1781-6.

364. Wijnands KA, Vink H, Briede JJ, et al. Citrulline a more suitable substrate than arginine to restore NO production and the microcirculation during endotoxemia. PloS one 2012; 7(5): e37439.

365. Jr. MS. Regulation of arginine availability and its impact on NO synthesis. In: L I, ed. Nitric oxide: biology and pathobiology San Diego: Academic Press; 2000: 187 $-97$.

366. Cynober L. Pharmacokinetics of arginine and related amino acids. The Journal of nutrition 2007; 137(6 Suppl 2): 1646S-9S.

367. Moinard C, Nicolis I, Neveux N, Darquy S, Benazeth S, Cynober L. Dose-ranging effects of citrulline administration on plasma amino acids and hormonal patterns in healthy subjects: the Citrudose pharmacokinetic study. The British journal of nutrition 2008; 99(4): 855-62.

368. Moinard C, Maccario J, Walrand S, et al. Arginine behaviour after arginine or citrulline administration in older subjects. The British journal of nutrition 2016; 115(3): 399-404.

369. Moinard C, Cynober L. Citrulline: a new player in the control of nitrogen homeostasis. The Journal of nutrition 2007; 137(6 Suppl 2): 1621S-5S.

370. Shen LJ, Beloussow K, Shen WC. Accessibility of endothelial and inducible nitric oxide synthase to the intracellular citrulline-arginine regeneration pathway. Biochemical pharmacology 2005; 69(1): 97-104.

371. Waugh WH, Daeschner CW, 3rd, Files BA, McConnell ME, Strandjord SE. Oral citrulline as arginine precursor may be beneficial in sickle cell disease: early phase two results. Journal of the National Medical Association 2001; 93(10): 363-71.

372. Orozco-Gutierrez JJ, Castillo-Martinez L, OreaTejeda A, et al. Effect of L-arginine or L-citrulline oral supplementation on blood pressure and right ventricular function in heart failure patients with 
preserved ejection fraction. Cardiology journal 2010; 17(6): 612-8.

373. Jourdan M, Nair KS, Carter RE, et al. Citrulline stimulates muscle protein synthesis in the postabsorptive state in healthy people fed a low-protein diet - A pilot study. Clinical nutrition 2015; 34(3): 449-56.

374. Sureda A, Cordova A, Ferrer MD, et al. Effects of Lcitrulline oral supplementation on polymorphonuclear neutrophils oxidative burst and nitric oxide production after exercise. Free radical research 2009; 43(9): 82835.

375. Suzuki T, Morita M, Kobayashi Y, Kamimura A. Oral L-citrulline supplementation enhances cycling time trial performance in healthy trained men: Double-blind randomized placebo-controlled 2-way crossover study. Journal of the International Society of Sports Nutrition 2016; 13: 6 .

376. Bouillanne O, Melchior JC, Faure C, et al. Impact of 3-week citrulline supplementation on postprandial protein metabolism in malnourished older patients: The Ciproage randomized controlled trial. Clinical nutrition 2019; 38(2): 564-74.

377. Ham DJ, Kennedy TL, Caldow MK, Chee A, Lynch GS, Koopman R. Citrulline does not prevent skeletal muscle wasting or weakness in limb-casted mice. The Journal of nutrition 2015; 145(5): 900-6.

378. Mirenayat MS, Moradi S, Mohammadi H, Rouhani MH. Effect of L-Citrulline Supplementation on Blood Pressure: a Systematic Review and Meta-Analysis of Clinical Trials. Current hypertension reports 2018; 20(11): 98.

379. Wise JK, Sena K, Vranizan K, et al. Temporal gene expression profiling during rat femoral marrow ablation-induced intramembranous bone regeneration. PloS one 2010; 5(10).

380. Lauing KL, Sundaramurthy S, Nauer RK, Callaci JJ. Exogenous activation of Wnt/beta-catenin signaling attenuates binge alcohol-induced deficient bone fracture healing. Alcohol and alcoholism 2014; 49(4): 399-408.

381. Alsousou J, Thompson M, Hulley P, Noble A, Willett $K$. The biology of platelet-rich plasma and its application in trauma and orthopaedic surgery: a review of the literature. The Journal of bone and joint surgery British volume 2009; 91(8): 987-96.

382. Kasten KR, Goetzman HS, Reid MR, et al. Divergent adaptive and innate immunological responses are observed in humans following blunt trauma. $B M C$ immunology 2010; 11: 4.

383. Laudi S, Donaubauer B, Busch T, et al. Low incidence of multiple organ failure after major trauma. Injury 2007; 38(9): 1052-8.

384.Visser T. Monitoring and Modulating of the Neutrophil Response [Dissertation]. Utrecht: Utrecht University; 2012.

385. Bastian OW, Koenderman L, Alblas J, Leenen LP, Blokhuis TJ. Neutrophils contribute to fracture healing by synthesizing fibronectin+ extracellular matrix rapidly after injury. Clinical immunology 2016; 164: 7884.

386. Kurose I, Anderson DC, Miyasaka M, et al. Molecular determinants of reperfusion-induced leukocyte adhesion and vascular protein leakage. Circulation research 1994; 74(2): 336-43.

387. Seekamp A, van Griensven M, Dhondt E, et al. The effect of anti-L-selectin (aselizumab) in multiple traumatized patients--results of a phase II clinical trial. Critical care medicine 2004; 32(10): 2021-8.

388. Lord JM, Midwinter MJ, Chen YF, et al. The systemic immune response to trauma: an overview of pathophysiology and treatment. Lancet 2014; 384(9952): 1455-65.

389. Mescher AL. Macrophages and fibroblasts during inflammation and tissue repair in models of organ regeneration. Regeneration 2017; 4(2): 39-53.

390. Juban G, Chazaud B. Metabolic regulation of macrophages during tissue repair: insights from skeletal muscle regeneration. FEBS letters 2017; 591(19): 3007 21.

391. Godwin JW, Pinto AR, Rosenthal NA. Chasing the recipe for a pro-regenerative immune system. Seminars in cell \& developmental biology 2017; 61: 71-9.

392. Udagawa N, Takahashi N, Akatsu T, et al. Origin of osteoclasts: mature monocytes and macrophages are capable of differentiating into osteoclasts under a suitable microenvironment prepared by bone marrowderived stromal cells. Proceedings of the National Academy of Sciences of the United States of America 1990; 87(18): 7260-4.

393. Boyle WJ, Simonet WS, Lacey DL. Osteoclast differentiation and activation. Nature 2003; 423(6937): 337-42.

394. McKibbin B. The biology of fracture healing in long bones. The Journal of bone and joint surgery British volume 1978; 60-B(2): 150-62.

395. El Khassawna T, Serra A, Bucher $\mathrm{CH}$, et al. T Lymphocytes Influence the Mineralization Process of Bone. Frontiers in immunology 2017; 8: 562.

396. Konnecke I, Serra A, El Khassawna T, et al. T and B cells participate in bone repair by infiltrating the fracture callus in a two-wave fashion. Bone 2014; 64: 155-65.

397. Sun G, Wang Y, Ti Y, Wang J, Zhao J, Qian H. Regulatory B cell is critical in bone union process through suppressing proinflammatory cytokines and stimulating Foxp3 in Treg cells. Clinical and experimental pharmacology \& physiology 2017; 44(4): 455-62.

398. Soderstrom K, Stein E, Colmenero P, et al. Natural killer cells trigger osteoclastogenesis and bone destruction in arthritis. Proceedings of the National Academy of Sciences of the United States of America 2010; 107(29): 13028-33.

399. Almeida CR, Ashkenazi A, Shahaf G, Kaplan D, Davis DM, Mehr R. Human NK cells differ more in their KIR2DL1-dependent thresholds for HLA-Cw6-mediated inhibition than in their maximal killing capacity. PloS one 2011; 6(9): e24927.

400. Krampera M. Mesenchymal stromal cell 'licensing': a multistep process. Leukemia 2011; 25(9): 1408-14.

401. Ren G, Zhang L, Zhao X, et al. Mesenchymal stem cell-mediated immunosuppression occurs via concerted action of chemokines and nitric oxide. Cell stem cell 2008; 2(2): 141-50. 
402. Han X, Yang Q, Lin L, et al. Interleukin-17 enhances immunosuppression by mesenchymal stem cells. Cell death and differentiation 2014; 21(11): 1758-68.

403. Polchert D, Sobinsky J, Douglas G, et al. IFN-gamma activation of mesenchymal stem cells for treatment and prevention of graft versus host disease. European journal of immunology 2008; 38(6): 1745-55.

404. Sadallah S, Eken C, Schifferli JA. Ectosomes as modulators of inflammation and immunity. Clinical and experimental immunology 2011; 163(1): 26-32.

405. Chen G, Li P, Liu Z, et al. Enrichment of miR-126 enhances the effects of endothelial progenitor cellderived microvesicles on modulating MC3T3-E1 cell function via Erk1/2-Bcl-2 signalling pathway. Prion 2019; 13(1): 106-15.

406. Baksh D, Yao R, Tuan RS. Comparison of proliferative and multilineage differentiation potential of human mesenchymal stem cells derived from umbilical cord and bone marrow. Stem cells 2007; 25(6): 1384-92.

407. Li G, White G, Connolly C, Marsh D. Cell proliferation and apoptosis during fracture healing. Journal of bone and mineral research : the official journal of the American Society for Bone and Mineral Research 2002; 17(5): 7919.

408. Meijer GJ, de Bruijn JD, Koole R, van Blitterswijk CA. Cell-based bone tissue engineering. PLoS medicine 2007; 4(2): e9.

409. Dang PN, Herberg S, Varghai D, et al. Endochondral Ossification in Critical-Sized Bone Defects via Readily Implantable Scaffold-Free Stem Cell Constructs. Stem cells translational medicine 2017; 6(7): 1644-59.

410. Orth M, Shenar AK, Scheuer C, et al. VEGF-loaded mineral-coated microparticles improve bone repair and are associated with increased expression of epo and RUNX-2 in murine non-unions. Journal of orthopaedic research : official publication of the Orthopaedic Research Society 2019; 37(4): 821-31.

411. Zhang Y, Hao Z, Wang P, et al. Exosomes from human umbilical cord mesenchymal stem cells enhance fracture healing through HIF-1alpha-mediated promotion of angiogenesis in a rat model of stabilized fracture. Cell proliferation 2019; 52(2): e12570.

412. Hao ZC, Lu J, Wang SZ, Wu H, Zhang YT, Xu SG. Stem cell-derived exosomes: A promising strategy for fracture healing. Cell proliferation 2017; 50(5).

413. Furuta T, Miyaki S, Ishitobi H, et al. Mesenchymal Stem Cell-Derived Exosomes Promote Fracture Healing in a Mouse Model. Stem cells translational medicine 2016; 5(12): 1620-30.

414. Xie Y, Chen Y, Zhang L, Ge W, Tang P. The roles of bone-derived exosomes and exosomal microRNAs in regulating bone remodelling. Journal of cellular and molecular medicine 2017; 21(5): 1033-41.

415. Newman RJ, Stone MH, Mukherjee SK. Accelerated fracture union in association with severe head injury. Injury 1987; 18(4): 241-6.

416. Perkins R, Skirving AP. Callus formation and the rate of healing of femoral fractures in patients with head injuries. The Journal of bone and joint surgery British volume 1987; 69(4): 521-4.
417. Rockwood CA, DP. G. Bone and joint healing. Rockwood and Green's Fractures in Adults,. 6 ed. Philadelphia: Lippincott; 2006: 297-312.

418. Giannoudis PV, Mushtaq S, Harwood P, et al. Accelerated bone healing and excessive callus formation in patients with femoral fracture and head injury. Injury 2006; 37 Suppl 3: S18-24.

419. Sternberg EM. Neural regulation of innate immunity: a coordinated nonspecific host response to pathogens. Nature reviews Immunology 2006; 6(4): 31828.

420. Schunkert H. Interaktion neurohumoraler Systeme bei der Blutdruckregulation. Deutsche Zeitschrift fur Sportmedizin 2002; 53(4).

421. Rosas-Ballina M, Tracey KJ. Cholinergic control of inflammation. Journal of internal medicine 2009; 265(6): 663-79.

422. Khallaf FG, Kehinde EO, Hussein S. Bone Healing and Hormonal Bioassay in Patients with Long-Bone Fractures and Concomitant Head Injury. Medical principles and practice : international journal of the Kuwait University, Health Science Centre 2016; 25(4): 336-42.

423. Kalb A, von Haefen C, Sifringer M, et al. Acetylcholinesterase inhibitors reduce neuroinflammation and -degeneration in the cortex and hippocampus of a surgery stress rat model. PloS one 2013; 8(5): e62679.

424. Borovikova LV, Ivanova S, Zhang M, et al. Vagus nerve stimulation attenuates the systemic inflammatory response to endotoxin. Nature 2000; 405(6785): 45862.

425. Parrish WR, Rosas-Ballina M, Gallowitsch-Puerta M, et al. Modulation of TNF release by choline requires alpha7 subunit nicotinic acetylcholine receptormediated signaling. Molecular medicine 2008; 14(9-10): 567-74.

426. Tracey KJ. Reflex control of immunity. Nature reviews Immunology 2009; 9(6): 418-28.

427. Huston JM. The vagus nerve and the inflammatory reflex: wandering on a new treatment paradigm for systemic inflammation and sepsis. Surgical infections 2012; 13(4): 187-93.

428. Wessler I, Kirkpatrick CJ. Acetylcholine beyond neurons: the non-neuronal cholinergic system in humans. British journal of pharmacology 2008; 154(8): 1558-71.

429. En-Nosse M, Hartmann S, Trinkaus K, et al. Expression of non-neuronal cholinergic system in osteoblast-like cells and its involvement in osteogenesis. Cell and tissue research 2009; 338(2): 203-15.

430. McCarthy ID, Andhoga M, Batten JJ, Mathie RT. Endothelium-dependent vasodilatation produced by the L-arginine/nitric oxide pathway in normal and ischemic bone. Acta orthopaedica Scandinavica 1997; 68(4): 3618.

431. Kliemann K, Kneffel M, Bergen I, et al. Quantitative analyses of bone composition in acetylcholine receptor M3R and alpha7 knockout mice. Life sciences 2012; 91(21-22): 997-1002.

432. Udupa KN. The neurohumoral response to trauma. American journal of surgery 1978; 135(3): 403-5. 
433. Prasad GC, Wahi RS, Gupta RC, Udupa KN. Neurohumoral response after fracture. Indian journal of experimental biology 1974; 12(1): 25-6.

434. Hill EL, Elde R. Distribution of CGRP-, VIP-, D beta $\mathrm{H}-$, SP-, and NPY-immunoreactive nerves in the periosteum of the rat. Cell and tissue research 1991; 264(3): 469-80.

435. Hukkanen M, Konttinen YT, Rees RG, Santavirta S, Terenghi G, Polak JM. Distribution of nerve endings and sensory neuropeptides in rat synovium, meniscus and bone. International journal of tissue reactions 1992; 14(1): 1-10.

436. Hill EL, Turner R, Elde R. Effects of neonatal sympathectomy and capsaicin treatment on bone remodeling in rats. Neuroscience 1991; 44(3): 747-55.

437. Sandhu HS, Kwong-Hing A, Herskovits MS, Singh IJ. The early effects of surgical sympathectomy on bone resorption in the rat incisor socket. Archives of oral biology 1990; 35(12): 1003-7.

438. Kumagai H, Sakamoto H, Guggino S, Filburn CR, Sacktor B. Neurotransmitter regulation of cytosolic calcium in osteoblast-like bone cells. Calcified tissue international 1989; 45(4): 251-4.

439. Moore RE, Smith CK, 2nd, Bailey CS, Voelkel EF, Tashjian AH, Jr. Characterization of beta-adrenergic receptors on rat and human osteoblast-like cells and demonstration that beta-receptor agonists can stimulate bone resorption in organ culture. Bone and mineral 1993; 23(3): 301-15.

440. Bjurholm A, Kreicbergs A, Schultzberg M, Lerner UH. Neuroendocrine regulation of cyclic AMP formation in osteoblastic cell lines (UMR-106-01, ROS 17/2.8, MC3T3-E1, and Saos-2) and primary bone cells. Journal of bone and mineral research : the official journal of the American Society for Bone and Mineral Research 1992; 7(9): 1011-9.

441. Oshima T, Yoshimoto T, Yamamoto S, Kumegawa M, Yokoyama C, Tanabe T. cAMP-dependent induction of fatty acid cyclooxygenase mRNA in mouse osteoblastic cells (MC3T3-E1). The Journal of biological chemistry 1991; 266(21): 13621-6.

442. Grills BL, Schuijers JA, Ward AR. Topical application of nerve growth factor improves fracture healing in rats. Journal of orthopaedic research : official publication of the Orthopaedic Research Society 1997; 15(2): 235-42.

443. Togari A, Arai M, Kondo H, Kodama D, Niwa Y. The neuro-osteogenic network: The sympathetic regulation of bone resorption. Japanese Dental Science Review 2012; 48(November): 61 - 70.

444. Chen CI, Yeh JS, Tsao NW, et al. Association between renin-angiotensin-aldosterone system blockade and future osteoporotic fracture risk in hypertensive population: A population-based cohort study in Taiwan. Medicine 2017; 96(46): e8331.

445. Elenkov IJ, Wilder RL, Chrousos GP, Vizi ES. The sympathetic nerve--an integrative interface between two supersystems: the brain and the immune system. Pharmacological reviews 2000; 52(4): 595-638.

446. Miksa M, Das P, Zhou M, et al. Pivotal role of the alpha(2A)-adrenoceptor in producing inflammation and organ injury in a rat model of sepsis. PloS one 2009; 4(5): e5504.
447. Spengler RN, Allen RM, Remick DG, Strieter RM, Kunkel SL. Stimulation of alpha-adrenergic receptor augments the production of macrophage-derived tumor necrosis factor. Journal of immunology 1990; 145(5): 1430-4.

448. Hasko G, Nemeth ZH, Szabo C, Zsilla G, Salzman AL, Vizi ES. Isoproterenol inhibits Il-10, TNF-alpha, and nitric oxide production in RAW 264.7 macrophages. Brain research bulletin 1998; 45(2): 183-7.

449. Siegmund B, Eigler A, Hartmann G, Hacker U, Endres S. Adrenaline enhances LPS-induced IL-10 synthesis: evidence for protein kinase A-mediated pathway. International journal of immunopharmacology 1998; 20(1-3): 57-69.

450. Fukumoto S, Shimizu Y. Fibroblast growth factor 23 as a phosphotropic hormone and beyond. Journal of bone and mineral metabolism 2011; 29(5): 507-14.

451. Razzaque MS, Lanske B. The emerging role of the fibroblast growth factor-23-klotho axis in renal regulation of phosphate homeostasis. The Journal of endocrinology 2007; 194(1): 1-10.

452. Corley DA, Kubo A, Zhao W, Quesenberry C. Proton pump inhibitors and histamine- 2 receptor antagonists are associated with hip fractures among at-risk patients. Gastroenterology 2010; 139(1): 93-101.

453. Woiciechowsky C, Schoning B, Lanksch WR, Volk HD, Docke WD. Mechanisms of brain-mediated systemic anti-inflammatory syndrome causing immunodepression. Journal of molecular medicine 1999; 77(11): 769-80.

454. John CD, Buckingham JC. Cytokines: regulation of the hypothalamo-pituitary-adrenocortical axis. Current opinion in pharmacology 2003; 3(1): 78-84.

455. Bornstein SR, Chrousos GP. Clinical review 104: Adrenocorticotropin (ACTH)- and non-ACTH-mediated regulation of the adrenal cortex: neural and immune inputs. The Journal of clinical endocrinology and metabolism 1999; 84(5): 1729-36.

456. Matejec R, Locke G, Muhling J, et al. Release of melanotroph- and corticotroph-type proopiomelanocortin derivatives into blood after administration of corticotropin-releasing hormone in patients with septic shock without adrenocortical insufficiency. Shock 2009; 31(6): 553-60.

457. Munford RS, Pugin J. The crucial role of systemic responses in the innate (non-adaptive) host defense. Journal of endotoxin research 2001; 7(4): 327-32.

458. Wang L, Cheng J, Hua Z, et al. alpha-melanocyte stimulating hormone (alpha-MSH) promotes osteoblast differentiation of MC3T3-E1 cells. European journal of pharmacology 2019; 844: 1-8.

459. Ishizu H, Sekiguchi T, Ikari T, et al. alphaMelanocyte-stimulating hormone promotes bone resorption resulting from increased osteoblastic and osteoclastic activities in goldfish. General and comparative endocrinology 2018; 262: 99-105.

460. Besedovsky H, del Rey A, Sorkin E, Dinarello CA. Immunoregulatory feedback between interleukin-1 and glucocorticoid hormones. Science 1986; 233(4764): 652-4.

461. Unger $\mathrm{T}$. The role of the renin-angiotensin system in the development of cardiovascular disease. The 
American journal of cardiology 2002; 89(2A): 3A-9A; discussion 10A.

462. Vejakama P, Thakkinstian A, Lertrattananon D, Ingsathit A, Ngarmukos C, Attia J. Reno-protective effects of renin-angiotensin system blockade in type 2 diabetic patients: a systematic review and network metaanalysis. Diabetologia 2012; 55(3): 566-78.

463. Putnam K, Shoemaker R, Yiannikouris F, Cassis LA. The renin-angiotensin system: a target of and contributor to dyslipidemias, altered glucose homeostasis, and hypertension of the metabolic syndrome. American journal of physiology Heart and circulatory physiology 2012; 302(6): H1219-30.

464. Asaba Y, Ito M, Fumoto T, et al. Activation of reninangiotensin system induces osteoporosis independently of hypertension. Journal of bone and mineral research : the official journal of the American Society for Bone and Mineral Research 2009; 24(2): 241-50.

465. Paul M, Poyan Mehr A, Kreutz R. Physiology of local renin-angiotensin systems. Physiological reviews 2006; 86(3): 747-803.

466. Shimizu H, Nakagami H, Osako MK, et al. Angiotensin II accelerates osteoporosis by activating osteoclasts. FASEB journal : official publication of the Federation of American Societies for Experimental Biology 2008; 22(7): 2465-75.

467. Li YC, Kong J, Wei M, Chen ZF, Liu SQ, Cao LP. 1,25Dihydroxyvitamin $D(3)$ is a negative endocrine regulator of the renin-angiotensin system. The Journal of clinical investigation 2002; 110(2): 229-38.

468. Agarwal D, Welsch MA, Keller JN, Francis J. Chronic exercise modulates RAS components and improves balance between pro- and anti-inflammatory cytokines in the brain of SHR. Basic research in cardiology 2011; 106(6): 1069-85.

469. Aubert J, Darimont C, Safonova I, Ailhaud G, Negrel R. Regulation by glucocorticoids of angiotensinogen gene expression and secretion in adipose cells. The Biochemical journal 1997; 328 ( Pt 2): 701-6.

470. Zhang YF, Qin L, Leung PC, Kwok TC. The effect of angiotensin-converting enzyme inhibitor use on bone loss in elderly Chinese. Journal of bone and mineral metabolism 2012; 30(6): 666-73.

471. Souza PP, Brechter AB, Reis RI, Costa CA, Lundberg P, Lerner UH. IL-4 and IL-13 inhibit IL-1beta and TNFalpha induced kinin B1 and B2 receptors through a STAT6-dependent mechanism. British journal of pharmacology 2013; 169(2): 400-12.

472. Srivastava S, Sharma K, Kumar N, Roy P. Bradykinin regulates osteoblast differentiation by Akt/ERK/NFkappaB signaling axis. Journal of cellular physiology 2014; 229(12): 2088-105.

473. Goncalves-Zillo TO, Pugliese LS, Sales VM, et al. Increased bone loss and amount of osteoclasts in kinin B1 receptor knockout mice. Journal of clinical periodontology 2013; 40(7): 653-60.

474. Kho C, Lee A, Hajjar RJ. Altered sarcoplasmic reticulum calcium cycling--targets for heart failure therapy. Nature reviews Cardiology 2012; 9(12): 717-33. 
Improved fracture healing in patients with concomitant traumatic brain injury: proven or not? Mediators of Inflammation

2015;2015:204842. doi: 10.1155/2015/204842. [Epub 2015 Mar 22] PMID: 25873754, PMCID: PMC4385630

\author{
Martijn Hofman ${ }^{1}$ \\ Guido Koopmans ${ }^{2}$ \\ Philipp Kobbe ${ }^{1}$ \\ Martijn Poeze ${ }^{3}$ \\ Hagen Andruszkow ${ }^{1}$ \\ Peter R.G. Brink ${ }^{3}$ \\ Hans-Christoph Pape ${ }^{1}$
}

1 Department of Orthopaedic Trauma and Reconstructive Surgery, University Medical Center RWTH Aachen, Germany

2 Algiax Pharmaceuticals GmbH, Erkrath, Germany

3 Department of Trauma Surgery, Maastricht University Medical Center + , the Netherlands 


\begin{abstract}
Over the last 3 decades, scientific evidence advocates an association between traumatic brain injury (TBI) and accelerated fracture healing. Multiple clinical and preclinical studies have shown an enhanced callus formation and an increased callus volume in patients, respectively, rats with concomitant TBI. Over time, different substances (cytokines, hormones, etc.) were in focus to elucidate the relationship between TBI and fracture healing. Until now, the mechanism behind this relationship is not fully clarified and a consensus on which substance plays the key role could not be attained in literature. In this review, we will give an overview of current concepts and opinions on this topic published in the last decade and both clinical and pathophysiological theories will be discussed.
\end{abstract}




\subsection{INTRODUCTION}

Outline of the review. In the last 3 decades, there are numerous studies published that either support or reject the hypothesis of enhanced callus development and fracture healing in patients with concomitant traumatic brain injury (TBI). Research on the development of heterotopic ossifications in paralytic patients goes back even further. The first studies on this subject, with the question whether fracture healing is influenced by accompanying TBI, were published in the early 1960s. Despite this history of studies, there is still no hard proof whether there is a relationship between TBI and enhanced callus formation. Moreover, the pathophysiological background of these phenomena is not clarified in the literature. A first review on these subjects was published by Morley and colleagues in $2005{ }^{1}$. They reviewed the literature on this topic until 2001, but they did not find a definite answer to their main question if traumatic brain injury results in accelerated fracture healing. The aim of our review is to evaluate the current status of knowledge and to compile an update on this topic. Evidence of a relationship between TBI and fracture healing could be important as a basis for further research to clarify the mechanism of normal and pathologic fracture healing.

\subsection{METHODS}

The following criteria were used to determine eligibility of a study to be included in this review. A literature search was carried out on Medline, Embase and Cochrane for studies published from January 2001 till December 2012 on the topic of fracture healing in subjects with concomitant traumatic brain injury. The following search terms were used in different combinations: "head trauma", "brain injury", "cerebral injury", "fracture healing", "bone healing", "pseudoarthrosis", and "peri-articular ossifications". The search was limited to manuscripts in English, German, or Dutch language. Letters to the editor and case reports were excluded. The references of selected studies were also pursued for articles that may have been missed via the electronic search.

\subsubsection{Study selection}

The title and abstract of all identified studies $(n=2880)$ were examined by one reviewer (Martijn Hofman). Then, the entire article was obtained and assessed for suitability by two of the authors (Martijn Hofman and Philipp Kobbe). Any issue pertaining to eligibility of studies was solved via discussion with the senior author (Hans-Christoph Pape). This resulted in 26 relevant articles, which were not included in the review of Morley and colleagues. Thirteen articles described clinical studies, of which 6 were prospective and 7 were retrospective cohort studies. An additional thirteen studies were preclinical (in vitro/in vivo) studies, including one review.

\subsection{FRACTURE HEALING}

Fracture healing occurs either by direct intramembranous healing or by indirect intramembranous and endochondral healing. Indirect fracture healing is the most common form and can be subdivided into multiple stages (Figure 2.1.). The first stage, named the Inflammation stage, starts with fracture and can last for ca. 5 days. In this stage, the fracture haematoma organizes and forms a link between the 
fracture fragments. This haematoma consists of blood cells, mesenchymal stem cells, fibroblasts, osteoclasts, osteoblasts, cytokines, growth factors, and other hormones.

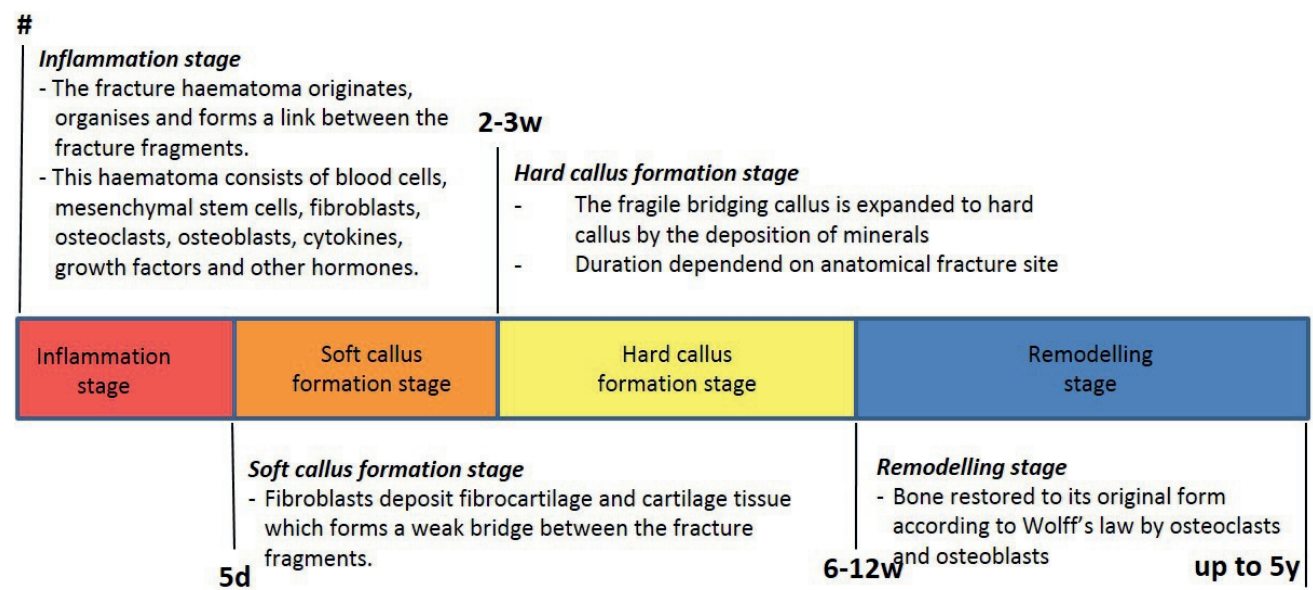

Figure 2.1. Indirect fracture healing.

The second stage is called soft (or bridging) callus formation stage and lasts for 2-3 weeks. Fibroblasts within the granulation haematoma deposit fibrocartilage and cartilage tissue which forms a weak bridge between the fracture fragments. The duration of the third stage of fracture healing, the hard (or medullary) callus formation stage, depends on the anatomical fracture site and will take between 6 and 12 weeks. In this stage, the fragile bridging callus is expanded to hard callus by the deposition of minerals. In the last stage of remodelling, which can last up to 5 years after injury, bone adaptation according to Wolff's law occurs. This law explains that, as a response to external loading, bone will be restored to its original form, involving bone resorption by osteoclasts and the formation of new bone by osteoblasts.

In the acute inflammatory reaction of fracture healing cytokines, such as tumour necrosis factor- $\alpha$ (TNF- $\alpha$ ), interleukin-1 (IL-1) and interleukin-6 (IL-6), are involved. TNF- $\alpha$ acts as a proinflammatory mediator and a chemotactic agent. Furthermore, it enhances the osteogenic differentiation of mesenchymal stem cells (MSC's) ${ }^{2}$. TNF- $\alpha$ peaks at about $24 \mathrm{~h}$ and returns to baseline at about $72 \mathrm{~h}$ after injury ${ }^{3}$. IL-1 is produced in the acute phase by macrophages in a biphasic mode. IL- 1 induces the IL6 -production by osteoblasts, the forming of cartilaginous callus, and the angiogenesis $2,4,5$.

IL-6 which is only active in the acute phase enhances also angiogenesis, the vascular endothelial growth factor- (VEGF-) production and osteoblasts and -clasts differentiation ${ }^{6}$.

After traumatic brain injury, the cytokine levels rise both in the cerebrospinal fluid and in the serum. Although the group of Kossmann found an approximately 10 to 100 times higher level of posttraumatic IL- 6 and IL-8 levels in the cerebrospinal fluid opposed to the plasma $7,8,9,10$, it is not clear whether this gradient is caused by a quick peripheral metabolism in the liver ${ }^{11}$ or by an initially higher local production in 
microglia, astrocytes, and macrophages 10,12. Furthermore, there are no studies finding an evidence of a direct correlation between the increased levels of cytokines and enhanced fracture healing or callus formation.

Indispensable for fracture healing is the recruitment of skeletal stem cells from surrounding tissues to the fracture site. Skeletal stem cells are mesenchymal stem cells which can differentiate to skeletal cell types including osteoblasts, chondrocytes, adipocytes, fibroblasts, and adventitial reticular cells ${ }^{13}$. These skeletal stem cells have three main functions, that is, they function as signalling centres, they provide a supportive microenvironment for haematopoiesis, and they stabilize and maintain the sinusoidal network within a fracture site. Although the mechanism of recruitment of these cells is still unclear, the recent opinion is that the stromal cellderived factor- 1 and its G-protein-coupled receptor CXCR-4 axis (SDF-1/CXCR-4axis) play an important role ${ }^{14-16}$. Other concepts of recruitment impute a role to transforming growth factor- $\beta$ (TGF- $\beta$ ), bone morphogenetic proteins (BMPs), insulin-like growth factor-1 (IGF-1), cellular retinol-binding protein 1 (CRBP-1), osteoblast stimulating factor (OSF-1), and hypoxia inducible factor- $1 \alpha(\mathrm{HIF}-1 \alpha){ }^{10}$.

Following activities of the MSC's at the fracture site, another immunological cascade occurs, in which the transforming growth factor- $\beta$ (TGF- $\beta$ ) superfamily, especially TGF- $\beta 2$, TGF- $\beta 3$, GDF-5, BMP-5, and BMP-6 seems to be involved 17,18. TGF- $\beta$ stimulates growth of cells of the osteoblastic lineage and acts as a chemo-attractant for osteoblasts. Another supposed function of TGF- $\beta$ is to increase the endogenous production of morphogens, such as bone morphogenetic proteins ${ }^{19}$. As members of the TGF- $\beta$ superfamily, three members of the BMP-subfamily enhance bone growth in peripheral locations, that is, BMP-2, BMP-4 and OP-1 (formerly BMP-7) 20-22. The work of Spector and colleagues shows that these three BMP's are expressed in early as well as advanced stages of bone healing and remodelling and as soon as mature bone has formed, the concentration of the BMP's normalize again ${ }^{23}$.

The revascularization of the fracture and callus site is regulated by the angiopoietindependent pathway in which the first vascular ingrowth occurs from existing vessels from the periosteum. However, the main part of the revascularization is regulated by the VEGF pathway, which transforms the cartilaginous avascular matrix into vascularized osseous tissue ${ }^{24}$. In the transformation of soft into hard callus, the Wntprotein family modulates the differentiation of MSC's into the osteoblastic lineage and later on the osteoblastic bone formation. Another cascade of immune-factors, such as macrophage colony-stimulating factor (M-CSF), receptor activator of nuclear factor kappa B ligand (RANKL), osteoprotegerin (OPG), and TNF- $\alpha$, starts the resorption of cartilage and the conversion in calcified bone tissue ${ }^{25}$.

The last remodelling stage of fracture healing is regulated by IL-1, TNF- $\alpha$, and some BMP's, especially BMP-2, and by the pressure applied to a crystalline environment ${ }^{26}$.

\subsection{TRAUMATIC BRAIN INJURY}

The prognosis of traumatic brain injury depends on both the primary and secondary brain damage. At the time of the initial traumatic impact on neurocranium and brain tissue, the primary brain injury originates and consists of concussion, contusion, shear injuries, lacerations, and axonal stretching ${ }^{27}$. In cases of severe primary brain injury, in which lesions of neurons, axons, and microglia cells occur, mortality rate is very high. 
Subsequent to the primary injury, a delayed complex immunological, biochemical, and physiological pathomechanism, which continues for several days to weeks, results in secondary brain damage ${ }^{27-29}$. This secondary brain damage is a multifactorial process which is caused and influenced by different processes, such as excitotoxicity, inflammation, oedema, cell death, mitochondrial damage, magnesium depletion, the production of free radicals, and damage to the blood brain barrier $27,28,30$.

\subsubsection{Excitotoxicity}

After primary and secondary brain injury excitotoxicity derives from the breakdown of neurons loaded with excitative neurotransmitters 28,31. Of these released neurotransmitters, glutamate is the most prominent neurotransmitter throughout the brain. This secretion of glutamate starts several minutes after the primary trauma, peaks about 10 minutes after trauma, and stays increased for several days 31. Through this glutamate discharge, an auto-destructive cascade is initiated by way of a calcium influx followed by a calcium overload, resulting in a stimulation of calcium-dependent enzymes, such as proteases, lipases, and endonucleases ${ }^{27}$.

\subsubsection{Inflammation}

Generally after trauma, classical or neurogenic, an inflammation cascade follows and the immune system is dysregulated, which influences the neurologic injury negatively ${ }^{28}$. Cytotoxic and inflammatory events with infiltration of leukocytes, macrophages, lymphocytes, and natural killer cells will occur. There are a lot of mediators, which influence the inflammation process after TBI. Amongst others, these are complement components, chemokines, and cytokines ${ }^{32}$.

The complement system is upregulated after TBI by passive leakage across the damaged BBB or by intracerebral synthesis ${ }^{33-35}$. As a "first line of defence", this system promotes the inflammation by recruiting proinflammatory molecules, phagocytosis, apoptosis, and damaging the BBB.

Other important proinflammatory modulators are chemokines. These heparinbinding proteins, which are produced by inflammatory cells, promote the infiltration of leukocytes in the traumatized brain tissue and thereby enhance the inflammatory reaction after TBI.

There are also many cytokines which have a proinflammatory role in the process after TBI. By the release of neuropeptides from sensory neurons, in case of neurogenic inflammation, extravasation of plasma, vasodilatation, and neuronal hypersensitivity results ${ }^{36}$. The most prominent neuropeptides involved are members of the bradykinin- and tachykinin-family of which CGRP (calcitonin generelated peptide) and substance $P$ are the most distinguished exponents ${ }^{37}$.

However, several studies in the last decade impute a dual role for some mediators supposing a proinflammatory and an anti-inflammatory effect in the post-TBI inflammatory process. This dual role is often demonstrated by a time-dependent pro- and anti-inflammatory characteristic of the different immune modulators, such as for IL-1, IL-6, TNF- $\alpha$, and chemokine (especially fractalkine $\left(\mathrm{CX}_{3} \mathrm{CL}_{1}\right)$ ) $^{38-41}$.

For TNF- $\alpha$, which appears to be synthetized in the brain tissue itself, as a endogenous response to TBI in the first few hours, such a dual function is demonstrated in several studies ${ }^{39,41-43}$. These studies demonstrate an early proinflammatory (1-2 d) and a 
late anti-inflammatory (2-4 wks.) role for TNF- $\alpha$. TNF- $\alpha$ increases vascular permeability leading to swelling of brain tissue and leukocyte infiltration. It also induces necrosis and apoptosis via intracellular pathways and it up-regulates the inflammatory mediator anaphylatoxin (C5a) on neurons ${ }^{44}$.

An important role in the inflammation after TBI is reserved for the interleukin-1family. IL-1 induces neuronal apoptosis and VEGF, an important mediator for the generation of posttraumatic oedema. The exact effect of IL-1, neurotoxic or neuroprotective, depends on the environment in which this cytokine resides. Also, IL-18 has raised concentrations in the cerebrospinal fluid after TBI 45 and by inhibiting both IL-1 and IL-18, the secondary brain damage after TBI could be reduced 41 . The dual role of the interleukin-family shows a proinflammatory phase in the first hours and days after TBI followed by a reparative phase lasting for days to months ${ }^{46}$.

Also IL-6, which is produced by neurons and macrophages early after TBI $(1 \mathrm{~h}$ after injury), can promote the inflammatory response but also has anti-inflammatory effects 42,43 . These anti-inflammatory effects are increased by the capacity of IL- 6 to inhibit TNF- $\alpha$ synthesis, induce nerve growth factor (NGF), promote survival and differentiation of neurons, and antagonize $N$-methyl-D-aspartate-mediated toxicity 42. Another aspect of IL-6, as a VEGF-agonist, is its quality to enhance angiogenesis and revascularization and thereby promote brain tissue repair ${ }^{47-50}$.

TGF- $\beta$, which increases within the first days after injury, may be produced by virtually all cells of the central nervous system (CNS). This growth factor counteracts the inflammation process by suppressing the release of IL-1, TNF- $\alpha$, IFN- $\gamma$ (Interferon- $\gamma$ ), oxygen radicals, MHC class II antigen expression, T-cell activation, and proliferation of various cells ${ }^{51-55}$. On the contrary, the chemotactic function of TGF- $\beta$ leads to leukocyte invasion and deposition of extracellular matrix (ECM) and scar tissue formation. These latter functions are more proinflammatory 56,57 .

\subsubsection{Oedema}

Although many factors contribute to the morbidity and mortality, the extent of cerebral oedema seems to be the supreme predictor of functional outcome after TBI 37,41,58. There are two forms of oedema identified after TBI. The vasogenic oedema, which is caused by the extravasation of fluid from the vasculature, has an early onset after trauma and is associated with an increased permeability of the BBB. The subsequent cytotoxic oedema originates from an osmotic shift of extracellular fluid to the intracellular compartment. The latter form has neurotoxic qualities and accounts for most of the brain swelling after TBI ${ }^{37,59}$. Key players in the development of postinjural oedema are aquaporins (AQPs), Matrix metalloproteinases (MMPs), and vasoactive agents. The expression of several AQPs, which are integral membrane proteins, is upregulated after TBI and promotes oedema formation ${ }^{60}$. The MMPs are zinc-dependent endopeptidases involved in the process of tissue remodelling following various pathologic conditions. The regulation of the MMP expression is complex and in cases of dysregulation by TBI, stroke, or neurodegeneration synaptic loss and breakdown of the BBB is identified, causing a vasogenic oedema and subsequent cell death ${ }^{61-64}$. The most important vasoactive agents are members of the bradykinin- and tachykinin-families and are produced in the neurogenic 
inflammation process. In particular, substance $\mathrm{P}$ is thought to enhance oedema formation ${ }^{37}$.

In case of posttraumatic oedema formation, the swelling of cells as well as parenchyma swells and leads to an elevation of the intracranial pressure (ICP), with a subsequential decrease of cerebral perfusion pressure (CPP). Eventually a herniation of the brain stem can occur ${ }^{37}$.

\subsubsection{Cell Death}

Cell death after TBI occurs in the first $24 \mathrm{~h}$ foremost via necrosis, in which swelling of mitochondria and other organelles and subsequent membrane degeneration occurs. In the subsequent days, cell death occurs also via apoptosis, in which DNA condensation and fragmentation, cell shrinkage, and the ultimate formation of apoptotic bodies occurs ${ }^{65}$. Another complicating factor is that the inhibition of one mechanism of cell death can exacerbate the other mechanism of cell death and vice versa 66,67 . Furthermore, beside the release of multiple proinflammatory modulators by the neurogenic inflammation cascade as described above, apoptotic and necrotic cells will set free multiple cytotoxic cytokines, growth factors, and interleukins, which will lead to a vicious circle of inflammation and cell death, which can last for months after the initial trauma ${ }^{41,42,68 .}$

\subsubsection{Mitochondrial Damage}

Brain and nerve tissue have a high energy demand and therefore the mitochondria are of utmost importance for the survival of these tissues. Severe injuries to the mitochondria can elicit devastating alterations to the mitochondrial respiration, respiratory coupling, and energy production ${ }^{69-71 .}$

\subsubsection{Magnesium Depletion}

A universal aspect of central nervous system (CNS) injury is a decrease of intracellular free magnesium, which plays normally a crucial role in normal cell function by regulating numerous physiological and biochemical processes within the cell ${ }^{72}$. Magnesium is a required cofactor in all energy producing and consuming reactions and over 300 enzymes involved in these processes are magnesiumdependent ${ }^{27}$. Beside these effects on enzymes, plasma membrane integrity, and ion channel activity are also influenced by magnesium ${ }^{73}$.

\subsubsection{Production of Free Radicals}

In the oxidative metabolism, free radicals are produces as normal by-products. The production of these highly reactive molecules is significantly enhanced by traumatic injuries ${ }^{74-76}$. Proteins, DNA, and lipids can be damaged by high concentrations of these free radicals, which lead to cell death via apoptosis ${ }^{77}$.

\subsubsection{Blood-brain-barrier damage}

If a cerebral mediator is released by TBI, which influences bone healing, it has to cross the blood brain barrier (BBB). The BBB is formed by the neurovascular unit, $a$ conjunction of cerebrovascular endothelial cells, pericytes, astrocytes, and the basal lamina ${ }^{78}$. The BBB strongly regulates the exchange of substances between plasma and the cerebral interstitium ${ }^{32}$. After TBI, there occurs a biphasic BBB disruption, 
with hyperpermeability in the beginning with a maximum at 4-6h after injury, followed by a transient restoration and a prolonged period of hyperpermeability $37,79,80$. Both small and large molecules are able to cross this barrier in and around the injury site ${ }^{81}$. The restoration of the BBB lasts from ca. 4 hours for large molecules to ca. 4-7 days for small molecules 37,81. There are many factors influencing the permeability of the BBB. In the beginning, the disruption is caused by a mechanical force, but in the later course of TBI other mediators are responsible for influencing the BBB, such as VEGF, angiopoietins, IL- $1 \beta$, IL-8, TNF- $\alpha$, reactive oxygen species, kinins, histamines, nitric oxide, elastase, matrix metalloproteinase (MMP) ${ }^{82-90}$. Furthermore, this permeability is also influenced by hypoxia after TBI, mostly after ca. $6 \mathrm{~h}$ post-injury and it can delay the restoration of the BBB by up to $72 \mathrm{~h}{ }^{91}$.

\subsection{REVIEW OF THE LITERATURE}

When we consider all studies on the topic of TBI and bone healing in the last decades, more than 50 different cells, hormones, growth factors, cytokines, chemokines, and so forth are reviewed and in a few cases there are some promising results (Table 2.1.).

Table 2.1. Investigated substances.

\begin{tabular}{|c|c|c|c|c|c|c|}
\hline \multicolumn{2}{|c|}{ Cytokines \& Growth factors } & \multirow{2}{*}{$\begin{array}{c}\text { Mesenchymal stem cells } \\
\text { hMSC }\end{array}$} & \multirow{2}{*}{$\begin{array}{c}\text { Genes } \\
\text { ALP }\end{array}$} & \multirow{2}{*}{$\begin{array}{c}\text { Hormones } \\
\text { Leptin }\end{array}$} & \multirow{2}{*}{$\begin{array}{l}\text { Proteins \& Enzymes } \\
\text { Alkaline phosphatase }\end{array}$} & \multirow{2}{*}{$\begin{array}{c}\text { Rest } \\
\text { Calcium }\end{array}$} \\
\hline IGF-1 & BMP4 & & & & & \\
\hline IGFBP-3 & OP-1 (BMP7) & C3H10T1/2-cells & CATK & Corticosteroids & Precursor type I collagen & Phosphate \\
\hline IL-1( $\beta)$ & $\operatorname{rhBMP}(-2 /-7)$ & MC3T3-cells & RUNK-2 & Calcitonin & CRP & \\
\hline IL-4 & VEGF & NIH3T3-cells & LacZ & CGRP(Calcitonin-gene- & Osterix protein (Sp7) & \\
\hline IL-5 & RANKL & hFOB-cells & & related peptide] & & \\
\hline IL-6 & OPG & BMSC & & Thyroxin & & \\
\hline IL-13 & M-CSF & PP1-cells & & Parathyroid hormone & & \\
\hline TNF- $\alpha$ & IGF-II & PP6-cells & & Androgens & & \\
\hline CCL-2 (MCP-1) & PDGF & & & Growth hormone & & \\
\hline CCL-20 & BFGF & & & Prolactin & & \\
\hline CXCL1 & & & & & & \\
\hline TGF- $\beta$ & & & & & & \\
\hline BMP2 & & & & & & \\
\hline
\end{tabular}

In the review of Morley and colleagues, they conclude that there is evidence for an accelerated osteogenesis associated with TBI. They found a relationship between TBI, rapid callus development, and stimulation of bone forming cells 1,92,93. However, they could not differentiate between heterotopic ossification and accelerated fracture healing 1 .

In the last decade, there were 26 studies on this topic after the publication of Morley and colleagues. About 50\% of these studies ${ }^{94-107}$ find evidence for increased callus formation in cases with concomitant TBI; the other studies are not conclusive.

There are only 7 of these studies which also postulate a possible working mechanism for this correlation. These are the studies we focus on in our review (Table 2.2.). 
Table 2.2. Studies with evidence for a certain mechanism of action.

\begin{tabular}{|c|c|c|c|c|c|}
\hline Study & $\begin{array}{c}\text { Year of } \\
\text { publication }\end{array}$ & $\begin{array}{l}\text { Clinical / } \\
\text { preclinical }\end{array}$ & $\begin{array}{c}\text { No. of } \\
\text { subjects }\end{array}$ & $\begin{array}{c}\text { Matched } \\
\text { control group }\end{array}$ & Suggested mechanism of action \\
\hline $\begin{array}{l}\text { Boes et al. } \\
{[96]}\end{array}$ & 2006 & Preclinical & $\mathrm{n}=43$ & yes & $\begin{array}{l}\text { Increased proliferation of mesenchymal stem cells, more specifically C3H10T1/2 } \\
\text { cells, due to brain injury }\end{array}$ \\
\hline $\begin{array}{l}\text { Wei et al. } \\
\text { [97] }\end{array}$ & 2008 & Preclinical & $\mathrm{n}=64$ & yes & Increased callus formation through an increased leptin level at the fracture site \\
\hline $\begin{array}{l}\text { Gautschi } \\
\text { et al. [98] }\end{array}$ & 2009 & $\begin{array}{l}\text { Combined clinical } \\
\text { and preclinical }\end{array}$ & $\mathrm{n}=61$ & yes & $\begin{array}{l}\text { Increased proliferation and differentiation of mesenchymal stem cells, caused by } \\
\text { the release of osteoinductive brain-derived factors }\end{array}$ \\
\hline $\begin{array}{l}\text { Cadosch } \\
\text { et al. [99] }\end{array}$ & 2009 & $\begin{array}{l}\text { Combined clinical } \\
\text { and preclinical }\end{array}$ & $\mathrm{n}=41$ & yes & Increased proliferation of the mesenchymal osteoprogenitor cell line hFOB1.19 \\
\hline $\begin{array}{l}\text { Zhang et } \\
\text { al. }[\underline{101}]\end{array}$ & 2009 & Preclinical & $\mathrm{n}=72$ & yes & $\begin{array}{l}\text { Increased secretion of calcitonin gene-related peptide in traumatic brain injury } \\
\text { group }\end{array}$ \\
\hline $\begin{array}{l}\text { Song et al. } \\
{[\underline{105}]}\end{array}$ & 2012 & Preclinical & $\mathrm{n}=24$ & yes & $\begin{array}{l}\text { Increased concentration of calcitonin gene-related peptide in serum released } \\
\text { from injured brain tissue }\end{array}$ \\
\hline $\begin{array}{l}\text { Yang et al. } \\
{[\underline{106}]}\end{array}$ & 2012 & Preclinical & $\mathrm{n}=36$ & yes & $\begin{array}{l}\text { Increased concentration of arachidonic acid in serum released from injured brain } \\
\text { tissue enhances BGLAP expression and proliferation of osteoblasts (MC3T3-E1 } \\
\text { cell line) }\end{array}$ \\
\hline
\end{tabular}

\subsubsection{Human Mesenchymal Stem Cells (hMSCs)}

Kanczler and Oreffo stress the importance of angiogenesis in combination with osteogenesis to optimize bone growth ${ }^{108}$. In fracture healing angiogenesis precedes osteogenesis. Xiao and colleagues show that bone marrow stromal stem cells (BMSCs) have the possibility to express both BMP-2 and VEGF and so these mesenchymal stem cells enhance fracture healing more explicit compared to the addition of any single factor ${ }^{109}$. Also Yamada and colleagues confirm the importance of a combination of osteogenic and angiogenic factors in the regeneration of bone. They found that a mixture of platelet-rich-plasma and mesenchymal stem cells can elicit better bone regeneration with good vascularization ${ }^{110}$. In the cascade after fractures, mesenchymal stem cells play an important role. These MSCs originate from bone marrow, periosteum, and so forth and are in case of a fracture generated to migrate to the fracture site in response to BMPs set free from the injured bone matrix 111,112 .

At the fracture site these MSCs produce different proteins and these proteins can differentiate the mesenchymal stem cells at their turn to enhance the fracture healing process ${ }^{23}$.

As multipotent cells, the MSCs can differentiate into different mesenchymal lineages which support the formation of distinct tissues, such as bone, cartilage, fat, tendon, muscle, and bone marrow stroma ${ }^{110,113}$.

This differentiation takes place under influence of different factors. Beside the proteins coming from the mesenchymal stem cells self as described above, Boes and colleagues propose an influence of unknown factors released by injured brain tissue, which exert their proliferative effect specific to mesenchymal stem cells ${ }^{96}$. In their in vitro analysis, they showed that the serum of rats with a fracture and concomitant TBI stimulated a multipotent mesenchymal stem cell line (C3H10T-cells) to proliferate at a significantly higher level $(\mathrm{p}=0.0002)$, resulting in a $76 \%$ increase in cells in the fracture/TBI group compared to the fracture-only group. They also investigated an osteoblastic (MC3T3-14-cells) and a fibroblastic (NIH 3T3-cells) cell line, but here they did not find any difference in proliferation rate ${ }^{96}$. Boes and colleagues compared the callus in rats with a femur fracture with concomitant TBI 
and without concomitant TBI. It was shown that after 21 days, the callus in the TBI and fracture group was reduced in diameter $(p=0.030)$, but it is significantly stiffer $(0.306 \mathrm{~N} / \mathrm{mm}$ compared with $0.120 \mathrm{~N} / \mathrm{mm} ; \mathrm{p}=0.02)$ than in the fracture-only group. The torsional strength was equal in both groups (258.4 Nm compared with 231.4 Nm; $\mathrm{p}=0.472)^{96}$.

The group of Cadosch and Gautschi investigated a human foetal osteoblastic mesenchymal stem cell line (hFOB1.19 cells) in an early stage of its differentiation 98,99. In an earlier study, they saw that the cerebrospinal fluid of patients with a traumatic brain injury had an osteoinductive potential and therefore they expected that any osteoinductive factor in the serum of patients with a traumatic brain injury would have a stimulating effect on the hFOB1.19 cells in vitro 114,115 . This potential reaches its maximum as soon as $6 \mathrm{~h}$ after injury, it remains at the same level for about 3 days and decreases after about 1 week ${ }^{98}$. They also observed an increased proliferation rate of osteoblasts exposed to sera from patients with TBI during the first week after injury ${ }^{98}$.

The time window of this effect is possibly explained by the traumatic disrupture of the blood brain barrier, permitting leakage of cerebrospinal fluid and the recovery of the blood brain barrier after about 1 week. Another explanation for the decreased osteoinductive potential after 1 week is a decreased production of osteogenic factors by the injured brain ${ }^{98,99}$.

Another result reported by the group of Cadosch and Gautschi was an increased expression of the osteoblastic differentiation marker gene in the serum of brain injured patients, such as ALP, CATK, RUNX-2, macrophage colony-stimulating factor and SP-7 99,116,117.

In the clinical part of their research, Gautschi and colleagues observed, in the TBI and fracture group in $41.7 \%$ of the cases, clinical and radiological evidence for hypertrophic callus formation. None of the cases in the fracture-only group developed hypertrophic callus. Moreover, Cadosch and colleagues found a positive correlation between callus ratio and proliferation of hFOB1.19 cells. On the contrary, they found an inverse correlation between fracture union time and callus ratio as well as between Glasgow Coma Scale (GCS) and callus ratio ${ }^{99}$.

In a recent in vitro study of Yang and colleagues increased levels of arachidonic acid (AA) were noted in serum metabolites of rats after TBI. They showed that in the presence of arachidonic acid the expression and proliferation of bone gamma carboxyglutamate protein (BGLAP or osteocalcin) is beneficially influenced and thereby the proliferation of the mouse osteoblastic cell line MC3T3-E1 was increased. They suggest a key role for arachidonic acid in the process of enhanced callus formation in rats with a TBI ${ }^{106}$.

\subsubsection{Leptin and CGRP}

In the metabolic, inflammatory, and neuroendocrine stress response occurring after TBI serum levels of leptin, an adipose-derived hormone, are significantly increased 97. The level of leptin is further influenced by different cytokines and hormonal factors, but the exact pathway of how leptin influences bone formation is not fully understood. It is postulated that the pro-inflammatory cytokine IL-1 increases rapidly after brain injury and this might cause an increased serum level of leptin 118,119 . 
Moreover, Wei and colleagues 97 found a positive correlation between leptin concentration in serum and volume of callus formation in patients with a fracture and a traumatic brain injury. Leptin, mainly produced by adipocytes, can influence bone metabolism by two pathways. In the first central pathway, leptin increases the sympathetic output by the hypothalamus which exerts an anti-osteogenic effect via $\beta 2$-adrenoceptors on osteoblasts. In the second peripheral pathway, leptin acts directly on peripheral tissues and then it has the opposite effect and promotes bone mineralisation and osteoblast-to-osteocyte differentiation ${ }^{97}$. They suggested several tracts for the serum level increase of leptin. First of all, the mobilization of free fatty acids as a result of hyper-metabolism in traumatic brain injury patients results in elevated serum leptin levels by a neuroendocrine feedback mechanism ${ }^{120}$. Secondly, hypoxia, caused by the adult respiratory distress syndrome or a pulmonary inflammatory response in traumatized patients, augments adipocyte expression of leptin ${ }^{121}$. Finally, the release of bone marrow at the fracture ends, containing mainly haematopoietic cells, induces leptin delivery at tissue level ${ }^{122}$. Furthermore, the complex neuroendocrine inflammatory response after TBI, with the release of multiple cytokines and hormones, influences the production and levels of leptin.

Wei and colleagues have shown that the serum leptin concentration reach a significant increased level only from the 4 th until the 12 th week after injury. This can be explained by the fact that in the acute posttraumatic period the stress response increases the sympathetic outflow, which down-regulates leptin expression and secretion by adipocytes. The secretion of leptin by adipocytes is also decreased due to the fasting state of a trauma patient in the acute posttraumatic stage. After the initial posttraumatic period, the peripheral effect of increasing leptin may outweigh the sympathetic inhibition of leptin on bone formation ${ }^{123,124}$.

The results of Wei and colleagues confirm the concept of Rayner and Trayhurn in 2001 and of Takeda and colleagues in 2002 that injury to the hypothalamus may result in a decreased sympathetic outflow, resulting in a subsequent decreased inhibition of peripheral leptin expression and a decreased anti-osteogenic effect via $\beta 2$-adrenoceptors on osteoblasts. In this way, injury to the hypothalamus contributes to the enhanced bone regeneration observed in rats with fractures and TBI ${ }^{97}$.

In their evaluation of callus formation, Wei and colleagues show a significantly increased callus volume in rats with TBI and fracture compared to rats with only a fracture from the 4 th till the 8th week after injury. This increase subsides at 12 weeks after injury, but in the histological analysis they can still proof thicker lamellar bone formation in the TBI and fracture group at 12 weeks after injury ${ }^{97}$.

Another possible mode of action of leptin on fracture healing is via calcitonin generelated peptide (CGRP). Zhang and colleagues ${ }^{125}$ showed in 2011 that peripheral administration of leptin alleviated injury-evoked brain damage by promoting CGRP expression, improving regional cerebral blood flow, and reducing local infarct volume and neurological deficits. Furthermore, leptin also promoted bcl-2 expression and suppressed caspase- 3 in vivo and vitro after injury. Administration of CGRP(8-37), an antagonist of the CGRP receptor, partly abolished the beneficial effects of leptin and restored the normal expression levels of bcl-2 and caspase- 3 in neurons, which indicated that leptin-induced protection of neurons was correlated with release of CGRP. 
Results of Song and colleagues showed in vivo a significantly elevated concentration of CGRP in rats with a femoral fracture and a concomitant traumatic brain injury. These concentrations were found both in the brain tissue and muscle tissue surrounding the fracture. They concluded that there was reciprocity between traumatic brain injury and enhanced fracture healing and they suppose that the CGRP is produced by the brain tissue. This conclusion was based on the observation that CGRP was expressed in the cerebral cortex and around the fracture site in the TBI and fracture group and not in the fracture-only group 105.

In their micro-CT-analysis of callus formation, they observed a significantly increased bone mineral density (BMD) and bone mineral content (BMC) of the newly formed callus in the TBI and fracture group compared to the fracture-only group in the 4th week after injury. The same measurement in the 8th week post-injury revealed that the BMD was indifferent and the BMC was significantly lower in the fracture-only group when compared to the TBI and fracture group. According to Song and colleagues, this indicated that fracture healing occurs earlier in cases with concomitant TBI 105 .

In 2000 Garcia-Castellano and colleagues ${ }^{126}$ already confirmed a great effect of CGRP on angioectasia to capillary, which is, as discussed before, of extreme importance for the acceleration of bone formation. According to Zhang and colleagues ${ }^{101}$ CGRP can exert this haemangiectasic role at the fracture site due to an axoplasmic transport of CGRP from the central nerve system.

\subsection{DISCUSSION}

For years physicians declare, according to their clinical experience, that callus formation/heterotopic ossification and fracture healing are accelerated in patients with accompanying traumatic brain injury. However, these statements are not based on hard evidence according to the literature, because the level of evidence in all of these clinical studies is not very high.

Before the review of Morley et al. ${ }^{1}$ in 2005 , there were only 5 clinical studies which found proof of enhanced callus formation in patients with TBI. These studies included one prospective study ${ }^{92}$ and 4 retrospective studies $93,127,128,129$.

In the last decade, six clinical studies were published, which found proof of an accelerated callus formation or fracture healing in patient with TBI and concomitant fractures. Five of them were prospective matched-control studies 95,98,99,100,106 with an average patient population of 65.2 (range 28-86) patients and one study was a retrospective, matched-control study ${ }^{94}$, with a patient population of 67 patients.

In the period before 2003 , there were 4 clinical studies which objected to this clinical experience because these studies did not find significant differences in callus formation or fracture healing between patients with and without traumatic brain injury. Of these 4 studies, there is only one prospective matched-control study ${ }^{130}$, but with 8 patients which is rather a case series. The other 3 studies are retrospective studies 131,132,133, of which only one study is matched-control.

In the last decade, there are no studies published which refute this enhanced callus formation in patients with concomitant TBI, but here publication bias must be considered. 
When we consider the studies which demonstrate a mechanism of action for the enhanced callus formation in patients with concomitant TBI, there are two mainstreams in the last decade.

The first mainstream is represented in $496,98,99,106$ of the 7 considered articles and puts the human mesenchymal stem cells/skeletal stem cells in focus.

In the study of Boes et al. ${ }^{96}$, adult rats were investigated, which were 7-9 months of age. The traumatic brain injury was administered when the animals showed signs of normal recovery after the administered femoral fracture and subsequent osteosynthesis, but the timing between administering the two lesions was not mentioned. The fact that the TBI and the fracture are not administered at the same time, as is the case in normal trauma patients, could be a confounding factor.

The biomechanical analysis and in vitro analysis of cell proliferation were both performed at 21 days after injury. This time point is very early because it is known that full recovery of mechanical properties of fractured femora in rats takes about 4 weeks in young and about 12 weeks in adult rats ${ }^{134}$. Nevertheless, they already found a significant increased stiffness of the fracture site in the fracture and TBI group compared to the fracture only group. This probably could be even more distinct in a later stage of fracture healing. Boes et al. ${ }^{96}$ conclude that the reduced callus diameter in the fracture and TBI group contradicts the concept that TBI increases endochondral ossification and they suggest that the fractures already have progressed into the remodelling phase. Regarding the early time point at which these investigations are performed, this does not, in our opinion, contradict the abovementioned concept because fracture healing has not finished yet and the remodelling phase probably has not started yet.

Also, the increase in the proliferation of the mesenchymal stem cell line C3H10T $1 / 2$ of $76 \%$ is an impressive result of the study group, which attribute this enhancement to a yet unknown soluble factor from injured neural tissue. Although the group of Boes et al. ${ }^{96}$ could not show an increased proliferation of a more advanced osteoblastic cell line (MC3T3-14), the group of Yang et al. did observe an increased proliferation of a more differentiated osteoblastic cell line of MC3T3-E1 cells. Of note, the analysis was performed with serum of TBI rats which was taken only at one time point, that is, $24 \mathrm{~h}$ after injury. In the bone healing process, the first few days are mainly determined by the inflammation phase in which the osteoblasts maybe play a subordinate role.

The results of the clinical part of the study of Gautschi et al. ${ }^{98}$ are convincing with $>40 \%$ of hypertrophic callus formation in the TBI and fracture group in comparison with the fracture only group, although the study groups are considerable small (respectively $n=12$ and $n=19$ ). They refer to pre-clinical studies of Boes et al. 96, Mandelin et al. ${ }^{116}$, and Camozzi et al. 117 in which proof is found for brain-derived factors with mitogenic and osteogenic effects on stromal stem cells and molecular mechanisms of sera from brain injured patients which mediate a mitogenic effect on osteoprogenitor cells. Cadosch et al. support these findings because they find a negative linear relationship between GCS and callus ratio on one site and time to union and callus ratio on the other site ${ }^{99}$. The proof of decreased time to union in patients with concomitant TBI is decisive in contrast with many other studies because time to union was an endpoint in their study and it was determined by two independent blinded radiologists. 
In their cell proliferation assay with primary human osteoblasts, they harvested the osteoblasts from 20 patients in which an osteosynthesis was performed. At what time these osteosyntheses were performed and from which group these samples were taken is not described. As a consequence, it remains unclear whether these osteoblasts were already stimulated in the body of the patients before harvesting. They also show that the osteoinductive effect of serum from TBI-patients on the hFOB-cell line is increased as soon as $6 \mathrm{~h}$ after injury until 3 days after injury. This effect fades after about 1 week. This could mean that this effect functions as a trigger for an enhanced callus formation. Whether this callus formation starts earlier through this trigger or evolves at a greater speed is still unsolved.

In the other study of Cadosch et al. ${ }^{102}$, the increased proliferation lasts even longer until the last measurement at $168 \mathrm{~h}$ after injury.

Because the observation period in both studies only lasted for 1 week after injury, it is unclear if this osteoinductive effect returns to normal levels after this week or that it has an analogous fluctuation as several cytokines in their dual function, with an additional effect later on in the fracture healing process. The fact that the disruption of the BBB lasts for about one week and therefore, the leakage of influencing factors produced by injured brain tissue will last for this period could be supportive to the findings of Gautschi and Cadosch 98,99,102.

Gautschi et al. 98 show an interesting pattern in the expression of osteoblastic markers (alkaline phosphatase (ALP), runt-related transcription factor 2 (RUNX-2), cathepsin K (CATK) and serine protease 7 (SP 7)), measured by the expression of mRNA in hFOB cells. ALP and RUNX-2 expression is significantly increased in the TBI and TBI and fracture group compared to the fracture-only group and the expression of SP 7 and CATK is significantly increased only in the TBI and fracture group compared to the TBI and the fracture-only group. This could mean that the expression of SP 7 and CATK is even more specific to the relationship between TBI and enhanced fracture healing than the expression of ALP and RUNX.

Furthermore, the fact that the results of the publications from Gautschi and Cadosch $98,99,102$ are very similar should be put in the perspective that both studies come from the same group of researchers.

The second mainstream is represented by Wei et al. ${ }^{97}$, Zhang et al. ${ }^{101}$, and Yang et al. 104 and they focus on the role of leptin and CGRP in the healing of fractures in patients with TBI.

In the study of Wei et al. ${ }^{97}$, which links serum leptin levels and leptin expression in callus cells to increased callus formation, they suggest a relationship between hypothalamic damage and reduced inhibition of peripheral leptin secretion and increased callus formation. This increase in leptin levels is reached only after 4 weeks after injury. In contrast to the studies on mesenchymal stem cells of Cadosch and Gautschi 98,99,102, Wei et al. ${ }^{97}$ extended the time slot of their histomorphological and histochemical analysis until 12 weeks after trauma. Considering the fracture healing process in adult rats, this is better suitable then a period of only 1 week.

Zhang et al. ${ }^{101}$ showed the link between increased leptin levels and an enhanced expression of CGRP and its haemangiectasic role at the fracture site.

Song et al. support these results and found proof for a correlation between increased fracture-healing tendency secondary to traumatic brain injury and high CGRP. They 
found a higher level of CGRP in the brain and muscles of traumatic brain injury rats and they suggest this CGRP is produced by brain tissue ${ }^{105}$.

According to their micro-CT analysis of callus formation, Song et al. conclude that in rats with concomitant TBI fracture healing occurs earlier as in the fracture-only group. This earlier healing appears in their population around the 4th week after injury and while these experiments are done with adult rats, this seems almost too early for fully developed callus formation ${ }^{134}$.

Another important finding of Zhang et al. is that different types of neural injury affected fracture healing in a different way. That is, peripheral nerve damage in combination with fractures can decelerate the healing process, and central nerve damage in combination with fractures can accelerate the healing rate. This increased healing rate as well as the inflammatory reaction following central neural injury is more intense and distinct for spinal cord injuries than for cerebral injuries 68,101, which could be of importance for future research.

\subsubsection{Future Perspectives}

The basic prove of enhanced fracture healing in patients with concomitant TBI is not yet substantiated by a large prospective clinical study. If this phenomenon can be proven, the interesting question is whether this mechanism is cellular or hormonally induced.

Important for future research is that the time course of mediator upregulation following TBI will be elucidated as well as for spinal cord injury by Donnelly and colleagues ${ }^{68}$. As the osteoinductive reaction after spinal cord injury is more pronounced as after TBI, it could be an interesting approach to look at accelerated callus formation in patients with spinal cord injury.

In case of the mesenchymal/skeletal stem cells as well as for the hormonal cascade, it is important to look at the fluctuation and influence within the fracture healing process over a longer period than 1 week after injury. Furthermore, the idea that enhanced callus formation in patients with fractures and concomitant TBI is nothing more than a form of heterotopic ossification, is also supported but not substantiated by several authors and studies in the last decades. This also could be an interesting reference point for further research.

\subsection{CONCLUSIONS}

In 2005 Morley and colleagues ${ }^{1}$ concluded that the question whether traumatic brain injury results in accelerated fracture union could not be answered at that time. Today, more than three decades after the first publications on this subject, the consensus in all published papers after 2005 is that traumatic brain injury indeed accelerates fracture healing. However, the greater part of studies on this topic are preclinical (in vitro/in vivo) studies, which indeed find some evidence for certain mechanisms, but the clinical studies with relatively small populations cannot, hitherto, support the hypothesis of accelerated fracture healing in patients with TBI. Moreover, in the last decade, the possibility of publication bias cannot be eliminated.

Furthermore, elucidation of the very complex mechanism of enhanced callus formation in patients with TBI is still in its infancy. Also in the last years there is very little revealed of the complex mechanism by which cytokines, chemokines, hormones 
and growth factors influence the signalling pathway leading to accelerated fracture healing.

The studies discussed in this review indicate that both serum and cerebrospinal fluid (CSF) from patients with fractures and concomitant TBI have osteoinductive potential. It also seems to be a consensus in the literature that these osteoinductive factors are released from the injured brain and from there spread in the body and to the fracture region.

In the literature published after the review of Morley and colleagues, there are two mainstreams which impute key roles in the complex mechanism of enhanced callus formation for mesenchymal stem cells on one side and for the leptin-CGRP-axis on the other side.

Nevertheless, because callus formation originates from a complex multifactorial cascade, it is possible that all described factors have their role in this phenomenon.

In our opinion, first of all these findings should be an incentive to design a large prospective clinical study to prove or reject the hypothesis of accelerated fracture healing in patients with concomitant TBI. The research on the pathophysiological relationship between TBI and callus formation should also be elaborated to reveal possible pathways of this assumed affiliation.

\subsection{ACKNOWLEDGEMENTS}

Conflict of interests. The authors declare that there is no conflict of interests regarding the publication of this paper.

\subsection{REFERENCES}

1. Morley J, Marsh S, Drakoulakis E, Pape HC, Giannoudis PV. Does traumatic brain injury result in accelerated fracture healing? Injury 2005; 36(3): 3638.

2. Kon T, Cho TJ, Aizawa T, et al. Expression of osteoprotegerin, receptor activator of NF-kappaB ligand (osteoprotegerin ligand) and related proinflammatory cytokines during fracture healing. Journal of bone and mineral research : the official journal of the American Society for Bone and Mineral Research 2001; 16(6): 1004-14.

3. Gerstenfeld LC, Cullinane DM, Barnes GL, Graves DT, Einhorn TA. Fracture healing as a post-natal developmental process: molecular, spatial, and temporal aspects of its regulation. Journal of cellular biochemistry 2003; 88(5): 873-84.

4. Lee SK, Lorenzo J. Cytokines regulating osteoclast formation and function. Current opinion in rheumatology 2006; 18(4): 411-8.

5. Sfeir C HL, Doll BA, Azari K, Hollinger JO. Fracture repair. In: Lieberman JR FG, ed. Bone regeneration and repair. Totowa, NJ: Humana Press; 2005: 21-44

6. Yang X, Ricciardi BF, Hernandez-Soria A, Shi Y, Pleshko Camacho N, Bostrom MP. Callus mineralization and maturation are delayed during fracture healing in interleukin-6 knockout mice. Bone 2007; 41(6): 928-36.

7. Kossmann T, Hans VH, Imhof HG, et al. Intrathecal and serum interleukin- 6 and the acute-phase response in patients with severe traumatic brain injuries. Shock 1995; 4(5): 311-7.
8. Kossmann T, Hans V, Imhof HG, Trentz O, Morganti-Kossmann MC. Interleukin-6 released in human cerebrospinal fluid following traumatic brain injury may trigger nerve growth factor production in astrocytes. Brain research 1996; 713(1-2): 143-52.

9. Kossmann T, Stahel PF, Lenzlinger PM, et al. Interleukin-8 released into the cerebrospinal fluid after brain injury is associated with blood-brain barrier dysfunction and nerve growth factor production. Journal of cerebral blood flow and metabolism : official journal of the International Society of Cerebral Blood Flow and Metabolism 1997; 17(3): 280-9.

10. Neugebauer E, Hensler T, Rose S, et al. [Severe craniocerebral trauma in multiple trauma. An assessment of the interaction of local and systemic mediator responses]. Der Unfallchirurg 2000; 103(2): 122-31.

11. Castell JV, Geiger T, Gross V, et al. Plasma clearance, organ distribution and target cells of interleukin-6/hepatocyte-stimulating factor in the rat. European journal of biochemistry / FEBS 1988; 177(2): 357-61.

12. Neidhardt $R$, Keel $M$, Steckholzer $U$, et al. Relationship of interleukin-10 plasma levels to severity of injury and clinical outcome in injured patients. The Journal of trauma 1997; 42(5): 863-70; discussion 70-1.

13. Friedenstein AJ, Petrakova KV, Kurolesova AI, Frolova GP. Heterotopic of bone marrow. Analysis of precursor cells for osteogenic and hematopoietic 
tissues. Transplantation 1968; 6(2): 230-47.

14. Granero-Molto F, Weis JA, Miga MI, et al. Regenerative effects of transplanted mesenchymal stem cells in fracture healing. Stem cells 2009; 27(8): 1887-98.

15. Kitaori T, Ito H, Schwarz EM, et al. Stromal cellderived factor 1/CXCR4 signaling is critical for the recruitment of mesenchymal stem cells to the fracture site during skeletal repair in a mouse model. Arthritis and rheumatism 2009; 60(3): 813-23.

16. Ma J, Ge J, Zhang S, et al. Time course of myocardial stromal cell-derived factor 1 expression and beneficial effects of intravenously administered bone marrow stem cells in rats with experimental myocardial infarction. Basic research in cardiology 2005; $100(3):$ 217-23.

17. Cho TJ, Gerstenfeld LC, Einhorn TA. Differential temporal expression of members of the transforming growth factor beta superfamily during murine fracture healing. Journal of bone and mineral research : the official journal of the American Society for Bone and Mineral Research 2002; 17(3): 513-20.

18. Marsell R, Einhorn TA. The role of endogenous bone morphogenetic proteins in normal skeletal repair. Injury 2009; 40 Suppl 3: S4-7.

19. Zellin G, Beck S, Hardwick R, Linde A. Opposite effects of recombinant human transforming growth factor-beta 1 on bone regeneration in vivo: effects of exclusion of periosteal cells by microporous membrane. Bone 1998; 22(6): 613-20.

20. Bentz H, Nathan RM, Rosen DM, et al. Purification and characterization of a unique osteoinductive factor from bovine bone. The Journal of biological chemistry 1989; 264(34): 20805-10.

21. Hammonds RG, Jr., Schwall R, Dudley A, et al. Bone-inducing activity of mature BMP-2b produced from a hybrid BMP-2a/2b precursor. Molecular endocrinology 1991; 5(1): 149-55.

22. Wang EA, Rosen V, D'Alessandro JS, et al. Recombinant human bone morphogenetic protein induces bone formation. Proceedings of the National Academy of Sciences of the United States of America 1990; 87(6): 2220-4.

23. Spector JA, Luchs JS, Mehrara BJ, Greenwald JA, Smith LP, Longaker MT. Expression of bone morphogenetic proteins during membranous bone healing. Plastic and reconstructive surgery 2001; 107(1): 124-34.

24. Keramaris NC, Calori GM, Nikolaou VS, Schemitsch EH, Giannoudis PV. Fracture vascularity and bone healing: a systematic review of the role of VEGF. Injury 2008; 39 Suppl 2: S45-57.

25. Marsell R, Einhorn TA. The biology of fracture healing. Injury 2011; 42(6): 551-5.

26. CAL B. . Biophysical principles affecting bone structure. In: GH B, ed. Biochemistry and Physiology of bone. New York: 2. Academic Press; 1971: 341-76

27. Vink R, Nimmo AJ. Novel therapies in development for the treatment of traumatic brain injury. Expert opinion on investigational drugs 2002; 11(10): 1375-86.

28. Hinson HE, Rowell S, Schreiber M. Clinical evidence of inflammation driving secondary brain injury: a systematic review. The journal of trauma and acute care surgery 2015; 78(1): 184-91.

29. Dalgard CL, Cole JT, Kean WS, et al. The cytokine temporal profile in rat cortex after controlled cortical impact. Frontiers in molecular neuroscience 2012; 5: 6.

30. Algattas H, Huang JH. Traumatic Brain Injury pathophysiology and treatments: early, intermediate, and late phases post-injury. International journal of molecular sciences 2014; 15(1): 309-41.

31. Bullock R, Zauner A, Woodward JJ, et al. Factors affecting excitatory amino acid release following severe human head injury. Journal of neurosurgery 1998; 89(4): 507-18.

32. Helmy A, De Simoni MG, Guilfoyle MR, Carpenter KL, Hutchinson PJ. Cytokines and innate inflammation in the pathogenesis of human traumatic brain injury. Progress in neurobiology 2011; 95(3): 352-72.

33. Gasque P, Dean YD, McGreal EP, VanBeek J, Morgan BP. Complement components of the innate immune system in health and disease in the CNS. Immunopharmacology 2000; 49(1-2): 171-86.

34. Woodruff TM, Ager RR, Tenner AJ, Noakes PG, Taylor SM. The role of the complement system and the activation fragment $\mathrm{C} 5 \mathrm{a}$ in the central nervous system. Neuromolecular medicine 2010; 12(2): 179-92.

35. Yanamadala V, Friedlander RM. Complement in neuroprotection and neurodegeneration. Trends in molecular medicine 2010; 16(2): 69-76.

36. Geppetti P, Bertrand C, Ricciardolo FL, Nadel JA. New aspects on the role of kinins in neurogenic inflammation. Canadian journal of physiology and pharmacology 1995; 73(7): 843-7.

37. Donkin JJ, Vink R. Mechanisms of cerebral edema in traumatic brain injury: therapeutic developments. Current opinion in neurology 2010; 23(3): 293-9.

38. Roberts I, Yates D, Sandercock P, et al. Effect of intravenous corticosteroids on death within 14 days in 10008 adults with clinically significant head injury (MRC CRASH trial): randomised placebo-controlled trial. Lancet 2004; 364(9442): 1321-8.

39. Scherbel U, Raghupathi R, Nakamura M, et al. Differential acute and chronic responses of tumor necrosis factor-deficient mice to experimental brain injury. Proceedings of the National Academy of Sciences of the United States of America 1999; 96(15): 8721-6.

40. Shohami E, Ginis I, Hallenbeck JM. Dual role of tumor necrosis factor alpha in brain injury. Cytokine \& growth factor reviews 1999; 10(2): 119-30.

41. Schmidt OI, Leinhase I, Hasenboehler E, Morgan SJ, Stahel PF. [The relevance of the inflammatory response in the injured brain]. Der Orthopade 2007; 36(3): 248, 50-8.

42. Morganti-Kossmann MC, Rancan M, Stahel PF, Kossmann T. Inflammatory response in acute traumatic brain injury: a double-edged sword. Current opinion in critical care 2002; 8(2): 101-5.

43. Lenzlinger PM, Morganti-Kossmann MC, Laurer HL, McIntosh TK. The duality of the inflammatory response to traumatic brain injury. Molecular neurobiology 2001; 24(1-3): 169-81.

44. Reid TR, Torti FM, Ringold GM. Evidence for two mechanisms by which tumor necrosis factor kills cells. The Journal of biological chemistry 1989; 264(8): 
4583-9.

45. Yatsiv I, Morganti-Kossmann MC, Perez D, et al. Elevated intracranial IL-18 in humans and mice after traumatic brain injury and evidence of neuroprotective effects of IL-18-binding protein after experimental closed head injury. Journal of cerebral blood flow and metabolism : official journal of the International Society of Cerebral Blood Flow and Metabolism 2002; 22(8): 971-8.

46. Schmidt OI, Heyde CE, Ertel W, Stahel PF. Closed head injury--an inflammatory disease? Brain research Brain research reviews 2005; 48(2): 388-99.

47. Wei LH, Kuo ML, Chen CA, et al. Interleukin-6 promotes cervical tumor growth by VEGF-dependent angiogenesis via a STAT3 pathway. Oncogene 2003; 22(10): 1517-27.

48. Yao JS, Zhai W, Young WL, Yang GY. Interleukin-6 triggers human cerebral endothelial cells proliferation and migration: the role for KDR and MMP-9. Biochemical and biophysical research communications 2006; 342(4): 1396-404.

49. Dore-Duffy P, Wang X, Mehedi A, Kreipke CW, Rafols JA. Differential expression of capillary VEGF isoforms following traumatic brain injury. Neurological research 2007; 29(4): 395-403.

50. Krum JM, Mani N, Rosenstein JM. Roles of the endogenous VEGF receptors flt- 1 and flk- 1 in astroglial and vascular remodeling after brain injury. Experimental neurology 2008; 212(1): 108-17.

51. Benveniste EN, Tang LP, Law RM. Differential regulation of astrocyte TNF-alpha expression by the cytokines TGF-beta, IL-6 and IL-10. International journal of developmental neuroscience : the official journal of the International Society for Developmental Neuroscience 1995; 13(3-4): 341-9.

52. Chao CC, Hu S, Sheng WS, Tsang M, Peterson PK. Tumor necrosis factor-alpha mediates the release of bioactive transforming growth factor-beta in murine microglial cell cultures. Clinical immunology and immunopathology 1995; 77(3): 358-65.

53. da Cunha A, Vitkovic L. Transforming growth factor-beta 1 (TGF-beta 1) expression and regulation in rat cortical astrocytes. Journal of neuroimmunology 1992; 36(2-3): 157-69.

54. Zhou D, Munster A, Winchurch RA. Pathologic concentrations of interleukin 6 inhibit $T$ cell responses via induction of activation of TGF-beta. FASEB journal : official publication of the Federation of American Societies for Experimental Biology 1991; 5(11): 25825.

55. Wahl SM. Transforming growth factor beta: the good, the bad, and the ugly. The Journal of experimental medicine 1994; 180(5): 1587-90.

56. Logan A, Berry M, Gonzalez AM, Frautschy SA, Sporn MB, Baird A. Effects of transforming growth factor beta 1 on scar production in the injured central nervous system of the rat. The European journal of neuroscience 1994; 6(3): 355-63.

57. Logan A, Berry M. Transforming growth factorbeta 1 and basic fibroblast growth factor in the injured CNS. Trends in pharmacological sciences 1993; 14(9): 337-42.

58. Clark RSB KP. Brain injury. Boston: Kluwer
Academic Publishers; 2001.

59. Barzo P, Marmarou A, Fatouros P, Hayasaki K, Corwin F. Contribution of vasogenic and cellular edema to traumatic brain swelling measured by diffusion-weighted imaging. Journal of neurosurgery 1997; 87(6): 900-7.

60. Manley GT, Fujimura M, Ma T, et al. Aquaporin-4 deletion in mice reduces brain edema after acute water intoxication and ischemic stroke. Nature medicine 2000; 6(2): 159-63.

61. Candelario-Jalil E, Yang Y, Rosenberg GA. Diverse roles of matrix metalloproteinases and tissue inhibitors of metalloproteinases in neuroinflammation and cerebral ischemia. Neuroscience 2009; 158(3): 983-94.

62. Ding JY, Kreipke CW, Schafer P, Schafer S, Speirs SL, Rafols JA. Synapse loss regulated by matrix metalloproteinases in traumatic brain injury is associated with hypoxia inducible factor-1alpha expression. Brain Res 2009; 1268: 125-34.

63. Rosenberg GA. Matrix metalloproteinases and their multiple roles in neurodegenerative diseases. The Lancet Neurology 2009; 8(2): 205-16.

64. Rosenberg GA, Yang Y. Vasogenic edema due to tight junction disruption by matrix metalloproteinases in cerebral ischemia. Neurosurg Focus 2007; 22(5): E4.

65. Pettmann B, Henderson CE. Neuronal cell death. Neuron 1998; 20(4): 633-47.

66. Lea PMt, Faden AI. Traumatic brain injury: developmental differences in glutamate receptor response and the impact on treatment. Mental retardation and developmental disabilities research reviews 2001; 7(4): 235-48.

67. Pohl D, Bittigau P, Ishimaru MJ, et al. N-Methyl-Daspartate antagonists and apoptotic cell death triggered by head trauma in developing rat brain. Proc Natl Acad Sci U S A 1999; 96(5): 2508-13.

68. Donnelly DJ, Popovich PG. Inflammation and its role in neuroprotection, axonal regeneration and functional recovery after spinal cord injury. Experimental neurology 2008; 209(2): 378-88.

69. Xiong Y, Gu Q, Peterson PL, Muizelaar JP, Lee CP. Mitochondrial dysfunction and calcium perturbation induced by traumatic brain injury. $J$ Neurotrauma 1997; 14(1): 23-34.

70. Harris LK, Black RT, Golden KM, Reeves TM, Povlishock JT, Phillips LL. Traumatic brain injuryinduced changes in gene expression and functional activity of mitochondrial cytochrome C oxidase. J Neurotrauma 2001; 18(10): 993-1009.

71. Verweij BH, Muizelaar JP, Vinas FC, Peterson PL, Xiong Y, Lee CP. Mitochondrial dysfunction after experimental and human brain injury and its possible reversal with a selective $\mathrm{N}$-type calcium channel antagonist (SNX-111). Neurological research 1997; 19(3): 334-9.

72. Vink R, McIntosh TK, Demediuk P, Weiner MW, Faden AI. Decline in intracellular free $\mathrm{Mg} 2+$ is associated with irreversible tissue injury after brain trauma. J Biol Chem 1988; 263(2): 757-61.

73. Bara M, Guiet-Bara A. Potassium, magnesium and membranes. Review of present status and new findings. Magnesium 1984; 3(4-6): 215-25. 
74. Cernak I, Savic VJ, Kotur J, Prokic V, Veljovic M, Grbovic D. Characterization of plasma magnesium concentration and oxidative stress following graded traumatic brain injury in humans. J Neurotrauma 2000; 17(1): 53-68.

75. Lewen A, Matz P, Chan PH. Free radical pathways in CNS injury. J Neurotrauma 2000; 17(10): 871-90.

76. Braughler JM, ED H. Involvement of lipid peroxidation in CNS injury. . In: Jane JA, Torner JC, Anderson DK, W Y, eds. Central Nervous System Trauma Status Report - 1991: Mary Ann Liebert, Inc., New York; 1992: 1-7.

77. Halestrap AP, Woodfield KY, Connern CP. Oxidative stress, thiol reagents, and membrane potential modulate the mitochondrial permeability transition by affecting nucleotide binding to the adenine nucleotide translocase. J Biol Chem 1997; 272(6): 3346-54.

78. Ballabh P, Braun A, Nedergaard M. The bloodbrain barrier: an overview: structure, regulation, and clinical implications. Neurobiology of disease 2004; 16(1): 1-13.

79. Baldwin SA, Fugaccia I, Brown DR, Brown LV, Scheff SW. Blood-brain barrier breach following cortical contusion in the rat. Journal of neurosurgery 1996; 85(3): 476-81.

80. Veltkamp R, Siebing DA, Sun L, et al. Hyperbaric oxygen reduces blood-brain barrier damage and edema after transient focal cerebral ischemia. Stroke; a journal of cerebral circulation 2005; 36(8): 1679-83.

81. Habgood MD, Bye N, Dziegielewska KM, et al. Changes in blood-brain barrier permeability to large and small molecules following traumatic brain injury in mice. The European journal of neuroscience 2007; 25(1): 231-8.

82. Armao D, Kornfeld M, Estrada EY, Grossetete M, Rosenberg GA. Neutral proteases and disruption of the blood-brain barrier in rat. Brain research 1997; 767(2): 259-64.

83. Butt AM. Effect of inflammatory agents on electrical resistance across the blood-brain barrier in pial microvessels of anaesthetized rats. Brain research 1995; 696(1-2): 145-50.

84. Carlson SL, Parrish ME, Springer JE, Doty K, Dossett L. Acute inflammatory response in spinal cord following impact injury. Experimental neurology 1998; 151(1): 77-88.

85. Ellis W. Pulsed subcutaneous electrical stimulation in spinal cord injury: preliminary results. Bioelectromagnetics 1987; 8(2): 159-64.

86. Nag S, Picard P, Stewart DJ. Increased immunolocalization of nitric oxide synthases during blood-brain barrier breakdown and cerebral edema. Acta neurochirurgica Supplement 2000; 76: 65-8.

87. Sarker MH, Easton AS, Fraser PA. Regulation of cerebral microvascular permeability by histamine in the anaesthetized rat. The Journal of physiology 1998; 507 ( Pt 3): 909-18.

88. Unterberg A, Wahl M, Baethmann A. Effects of free radicals on permeability and vasomotor response of cerebral vessels. Acta neuropathologica 1988; 76(3): 238-44.

89. Schnell L, Fearn S, Schwab ME, Perry VH, Anthony
DC. Cytokine-induced acute inflammation in the brain and spinal cord. Journal of neuropathology and experimental neurology 1999; 58(3): 245-54.

90. Mautes AE, Weinzierl MR, Donovan F, Noble LJ. Vascular events after spinal cord injury: contribution to secondary pathogenesis. Physical therapy 2000; 80(7): 673-87.

91. Tanno H, Nockels RP, Pitts LH, Noble LJ. Breakdown of the blood-brain barrier after fluid percussion brain injury in the rat: Part 2: Effect of hypoxia on permeability to plasma proteins. Journal of neurotrauma 1992; 9(4): 335-47.

92. Bidner SM, Rubins IM, Desjardins JV, Zukor DJ, Goltzman D. Evidence for a humoral mechanism for enhanced osteogenesis after head injury. The Journal of bone and joint surgery American volume 1990; 72(8): 1144-9.

93. Newman RJ, Stone MH, Mukherjee SK. Accelerated fracture union in association with severe head injury. Injury 1987; 18(4): 241-6.

94. Giannoudis PV, Mushtaq S, Harwood P, et al Accelerated bone healing and excessive callus formation in patients with femoral fracture and head injury. Injury 2006; 37 Suppl 3: S18-24.

95. Andermahr J, Elsner A, Brings AE, Hensler T, Gerbershagen $\mathrm{H}$, Jubel A. Reduced collagen degradation in polytraumas with traumatic brain injury causes enhanced osteogenesis. Journal of neurotrauma 2006; 23(5): 708-20.

96. Boes M, Kain M, Kakar S, et al. Osteogenic effects of traumatic brain injury on experimental fracturehealing. The Journal of bone and joint surgery American volume 2006; 88(4): 738-43.

97. Wei Y, Wang L, Clark JC, Dass CR, Choong PF. Elevated leptin expression in a rat model of fracture and traumatic brain injury. The Journal of pharmacy and pharmacology 2008; 60(12): 1667-72.

98. Gautschi OP, Cadosch D, Frey SP, Skirving AP, Filgueira L, Zellweger R. Serum-mediated osteogenic effect in traumatic brain-injured patients. ANZ journal of surgery 2009; 79(6): 449-55.

99. Cadosch D, Gautschi OP, Thyer M, et al. Humoral factors enhance fracture-healing and callus formation in patients with traumatic brain injury. The Journal of bone and joint surgery American volume 2009; 91(2): 282-8.

100. Lee JS, Ryu CH, Moon NH, Kim SJ, Park SY, Suh KT. Changes in serum levels of receptor activator of nuclear factor-kappaB ligand, osteoprotegerin, IL-6 and TNF-alpha in patients with a concomitant head injury and fracture. Archives of orthopaedic and trauma surgery 2009; 129(5): 711-8.

101. Zhang D, Zhang P, Wang Y, Han N, Tang C, Jiang B. The influence of brain injury or peripheral nerve injury on calcitonin gene-related peptide concentration variation and fractures healing process. Artificial cells, blood substitutes, and immobilization biotechnology 2009; 37(2): 85-91.

102. Cadosch D, Toffoli AM, Gautschi OP, et al. Serum after traumatic brain injury increases proliferation and supports expression of osteoblast markers in muscle cells. The Journal of bone and joint surgery American volume 2010; 92(3): 645-53. 
103. Ozan F, Yildiz H, Bora OA, Pekedis M, Ay Coskun G, Gore 0 . The effect of head trauma on fracture healing: biomechanical testing and finite element analysis. Acta orthopaedica et traumatologica turcica 2010; 44(4): 313-21.

104. Yang TY, Wang TC, Tsai YH, Huang KC. The effects of an injury to the brain on bone healing and callus formation in young adults with fractures of the femoral shaft. The Journal of bone and joint surgery British volume 2012; 94(2): 227-30.

105. Song Y, Bi L, Zhang Z, et al. Increased levels of calcitonin gene-related peptide in serum accelerate fracture healing following traumatic brain injury. Molecular medicine reports 2012; 5(2): 432-8.

106. Yang S, Ma Y, Liu Y, Que H, Zhu C, Liu S. Arachidonic acid: a bridge between traumatic brain injury and fracture healing. Journal of neurotrauma 2012; 29(17): 2696-705.

107. Huang W, Li Z, Li Z, Yang R. Does traumatic brain injury result in accelerated mandibular fracture healing? Journal of oral and maxillofacial surgery : official journal of the American Association of Oral and Maxillofacial Surgeons 2012; 70(9): 2135-42.

108. Kanczler JM, Oreffo RO. Osteogenesis and angiogenesis: the potential for engineering bone. European cells \& materials 2008; 15: 100-14.

109. Xiao C, Zhou H, Liu G, et al. Bone marrow stromal cells with a combined expression of BMP-2 and VEGF165 enhanced bone regeneration. Biomedical materials 2011; 6(1): 015013.

110. Yamada Y, Ueda M, Naiki T, Takahashi M, Hata K, Nagasaka T. Autogenous injectable bone for regeneration with mesenchymal stem cells and platelet-rich plasma: tissue-engineered bone regeneration. Tissue engineering 2004; 10(5-6): 95564.

111. Simmons DJ. Fracture healing perspectives. Clinical orthopaedics and related research 1985; (200): 100-13.

112. Yang LJ, Jin Y. Immunohistochemical observations on bone morphogenetic protein in normal and abnormal conditions. Clinical orthopaedics and related research 1990; (257): 249-56.

113. Opydo-Chanek M. Bone marrow stromal cells in traumatic brain injury (TBI) therapy: true perspective or false hope? Acta neurobiologiae experimentalis 2007; 67(2): 187-95.

114. Gautschi OP, Toffoli AM, Joesbury KA, Skirving AP, Filgueira L, Zellweger R. Osteoinductive effect of cerebrospinal fluid from brain-injured patients. Journal of neurotrauma 2007; 24(1): 154-62.

115. Renfree KJ, Banovac K, Hornicek FJ, Lebwohl NH, Villanueva PA, Nedd KJ. Evaluation of serum osteoblast mitogenic activity in spinal cord and head injury patients with acute heterotopic ossification. Spine 1994; 19(7): 740-6.

116. Mandelin J, Hukkanen M, Li TF, et al. Human osteoblasts produce cathepsin K. Bone 2006; 38(6): 769-77.

117. Camozzi V, Tossi A, Simoni E, Pagani F, Francucci CM, Moro L. Role of biochemical markers of bone remodeling in clinical practice. Journal of endocrinological investigation 2007; 30 (6 Suppl): 13-
7.

118. Fassbender K, Schneider S, Bertsch $T$, et al. Temporal profile of release of interleukin-1beta in neurotrauma. Neuroscience letters 2000; 284(3): 1358.

119. Janik JE, Curti BD, Considine RV, et al. Interleukin 1 alpha increases serum leptin concentrations in humans. The Journal of clinical endocrinology and metabolism 1997; 82(9): 3084-6.

120. Faggioni R, Moser A, Feingold KR, Grunfeld C. Reduced leptin levels in starvation increase susceptibility to endotoxic shock. The American journal of pathology 2000; 156(5): 1781-7.

121. Lolmede K, Durand de Saint Front V, Galitzky J, Lafontan M, Bouloumie A. Effects of hypoxia on the expression of proangiogenic factors in differentiated 3T3-F442A adipocytes. International journal of obesity and related metabolic disorders : journal of the International Association for the Study of Obesity 2003; 27(10): 1187-95

122. Lin J, Yan GT, Wang LH, Xue H, Hao XH, Zhang K. [Effect of long tubular bone fracture on serum levels of leptin, acute phase proteins and biochemical markers for organ functions]. Zhongguo wei zhong bing ji jiu yi $x u e=$ Chinese critical care medicine $=$ Zhongguo weizhongbing jijiuyixue 2006; 18(1): 19-23.

123. Mantzoros CS, Qu D, Frederich RC, et al. Activation of beta(3) adrenergic receptors suppresses leptin expression and mediates a leptin-independent inhibition of food intake in mice. Diabetes 1996; 45(7): 909-14.

124. Jeevanandam M, Begay CK, Petersen SR. Plasma leptin levels in trauma patients: effect of adjuvant recombinant human growth hormone in intravenously fed multiple trauma patients. JPEN Journal of parenteral and enteral nutrition 1998; 22(6): 340-6.

125. Zhang JY, Yan GT, Liao J, et al. Leptin attenuates cerebral ischemia/reperfusion injury partially by CGRP expression. European journal of pharmacology 2011; 671(1-3): 61-9.

126. Garcia-Castellano JM, Diaz-Herrera P, Morcuende JA. Is bone a target-tissue for the nervous system? New advances on the understanding of their interactions. The Iowa orthopaedic journal 2000; 20: 49-58.

127. Garland DE, Dowling V. Forearm fractures in the head-injured adult. Clinical orthopaedics and related research 1983; (176): 190-6.

128. Spencer RF. The effect of head injury on fracture healing. A quantitative assessment. The Journal of bone and joint surgery British volume 1987; 69(4): 525-8.

129. Perkins R, Skirving AP. Callus formation and the rate of healing of femoral fractures in patients with head injuries. The Journal of bone and joint surgery British volume 1987; 69(4): 521-4.

130. Beeton CA, Brooks RA, Chatfield D, Human M, Rushton N. Circulating levels of insulin-like growth factor-1 and insulin-like growth factor binding protein-3 in patients with severe head injury. The Journal of bone and joint surgery British volume 2002; 84(3): 434-9.

131. Garland DE, Toder L. Fractures of the tibial diaphysis in adults with head injuries. Clinical orthopaedics and related research 1980; (150): 198- 
202.

132. Garland DE, Rothi B, Waters RL. Femoral fractures in head-injuries adults. Clinical orthopaedics and related research 1982; (166): 219-25.

133. Pape HC, Lehmann U, van Griensven M, Gansslen A, von Glinski S, Krettek C. Heterotopic ossifications in patients after severe blunt trauma with and without head trauma: incidence and patterns of distribution. Journal of orthopaedic trauma 2001; 15(4): 229-37.

134. Ekeland A, Engesoeter LB, Langeland N. Influence of age on mechanical properties of healing fractures and intact bones in rats. Acta orthopaedica Scandinavica 1982; 53(4): 527-34 
Risk factors of non-union in intramedullary stabilized diaphyseal long bone fractures: Identifying the role of fracture stabilization strategies and concomitant injuries.

\title{
European Journal of Trauma \& Emergency Surgery
}

2020;10.1007/s00068-020-01335-y. doi: 10.1007/s00068-020-01335-

y. [published online ahead of print, 2020 Mar 5] PMID: 32140749

\author{
Martijn Hofman ${ }^{1}$ \\ Hagen Andruszkow ${ }^{1}$ \\ Frans Heyer ${ }^{2}$ \\ Philipp Kobbe ${ }^{1}$ \\ Frank Hildebrand ${ }^{1 \dagger}$ \\ Martijn Poeze ${ }^{2 \dagger}$
}

1 Department of Orthopaedic Trauma and Reconstructive Surgery, University Medical Center RWTH Aachen, Germany

2 Department of Trauma Surgery, Maastricht University Medical Center +, the Netherlands

$\dagger$ Authors contributed mutually 


\section{ABSTRACT}

Purpose: Concomitant chest injury is known to negatively affect bone metabolism and fracture healing, whereas traumatic brain injury (TBI) appears to have positive effects on bone metabolism. Osteogenesis can also be influenced by the timing of fracture stabilization. We aimed to identify how chest injuries, TBI, and fracture stabilization strategy influences the incidence of non-union.

Methods: Patients with long bone fractures of the lower extremities who had been treated between 2004 and 2014 were retrospectively analysed. Non-union was defined as fracture healing not occurring in the expected time and in which progression of healing nor successful union is expected without intervention. Diverse clinical and radiological parameters were statistically analysed using the Statistical Package for the Social Sciences (SPSS).

Results: The total number of operations before consolidation was an independent predictor (odds ratio $[\mathrm{OR}]=6.416, \mathrm{p}<0.001$ ) for the development of non-union in patients with long bone fractures. More specifically, patients treated according to the damage control orthopaedics (DCO) principle had a significantly higher risk of developing a non-union than patients treated according to the early total care (ETC) principle (OR $=7.878, \mathrm{p}=0.005$ ). Concomitant chest injury and TBI could not be identified as influencing factors for non-union development.

Conclusion: Our results indicate that the number of operations performed in patients with long bone fractures should be kept as low as possible and that the indication for and the timing of DCO treatment should be meticulously noted to minimize the risk of non-union development. 


\subsection{INTRODUCTION}

Fracture healing depends on the interactions of many biomechanical and biological factors 1 . Disturbances in this process might result in non-union with an overall incidence of $1.5-10 \%$, increasing up to $40 \%$ in case of open fractures. In particular, non-unions of the lower extremities have been identified to significantly impair the post-traumatic quality of life ${ }^{2}$, and have been associated with high direct and indirect costs ${ }^{3,4}$.

The risk factors for non-unions might either arise from injury characteristics, patient-specific factors, or from parameter associated with surgical fracture stabilization 5,6 . Interactions between local and systemic inflammatory responses have been considered as the potential reasons for delayed fracture healing in chest trauma, ${ }^{6}$. In contrast, TBI seems to be positively correlated with osteogenesis ${ }^{7-9}$. However, this association has not been found in all studies 10,11. Furthermore, potential pathophysiological mechanisms for TBI-related impact on osteogenesis seem to be multifactorial (humoral, hormonal, and cellular) and are far from clarified $7,12,13$.

Because of the enormous incapacitating effect of non-unions on the physical and mental health of patients, knowledge about the relevance of potential risk factors is of upmost importance. As the impact of several patient- (e.g. abuse of substances, long-term use of steroids) and injury-specific (e.g. open fracture Type III) factors has already been well described ${ }^{5}$, we focused on the influence of chest injury, TBI and fracture stabilization strategies in patients with long bone fractures of the lower extremities. Identifying the risk and predictive factors of non-union can help further develop prophylactic and therapeutic strategies for its treatment.

\subsection{MATERIALS AND METHODS}

\subsubsection{Study design and exclusion criteria}

We retrospectively analysed patients with diaphyseal femoral or tibial fractures who had been admitted and treated definitively with a reamed intramedullary locking nail at the department of Orthopaedic Trauma and Reconstructive Surgery, University Hospital RWTH Aachen (Germany), or the Department of Trauma Surgery, Maastricht University Medical Centre (The Netherlands) between 2004 and 2014. Clinical records and X-rays were retrieved for analysis. The patients' clinical course was followed until the last outpatient appointment. Patients who developed a non-union were placed in the NU group and those with normal fracture healing were placed in the control group. Non-union was defined as fracture healing not occurring in the expected time and in which progression of healing nor successful union is expected without intervention.

To restrict the number of previously described factors influencing non-union development and to focus on chest injury, TBI, and fracture stabilization strategy (ETC vs. DCO) as the influencing factors, we applied the following exclusion criteria: $17<$ age (years) < 80, substance abuse

(alcohol, tobacco, drugs), morbid obesity (BMI > 30), mental deficiency, pregnancy, long-term use of steroids, bisphosphonates, or thyroxin, lost to follow-up <1 year after trauma, Severe soft tissue damage (Gustilo \& Anderson $>2$ ), Comminuted fracture, Bone defect $>3 \mathrm{~cm}$, Pathological fractures, fractures of an adjacent joint, 
bilateral fractures, definitive treatment other than reamed intramedullary locking nail, and primary definitive treatment elsewhere.

\subsubsection{Treatment algorithm}

All the patients were managed according to the principles of Advanced Trauma Life Support ${ }^{\circledR}\left(\right.$ ATLS $\left.^{\circledR}\right)$ and the S3 guidelines on the treatment of patients with severe injuries. For fracture treatment, patients underwent ETC with an antegrade intramedullary reamed locking nail, and if necessary, DCO at the earliest possible opportunity with an external fixator, which was subsequently converted to definitive osteosynthesis as soon as it was tolerated by the patient's clinical condition. Intravenous antibiotic prophylaxis was given in closed fractures as a single dose and for 3 days in open fractures. As soon as their clinical state allowed it, patients were mobilized with partial and consecutive increase to full weight-bearing, according to the fracture type. After discharge, the patients were seen in the outpatient clinic at 2 , $6,12,26$, and 52 weeks postoperatively. If union was not achieved at that time point, further controls took place until union was achieved or a revision was indicated.

\subsubsection{General health status and injury severity}

The general health status of the patients was estimated according to the American College of Anaesthesiologists (ASA) classification system and the Charlson comorbidity index (CCI) ${ }^{14}$, which calculates an estimated relative risk of death based on the patient's age, cardiopulmonary and cerebro-vascular condition, the presence of metabolic, gastrointestinal and infectious diseases as well as malignancies. Overall injury severity was determined with the 2005 revised edition of the Abbreviated Injury Scale (AIS) and summarized to the Injury Severity Score (ISS) ${ }^{15}$.

\subsubsection{Classification of chest injury}

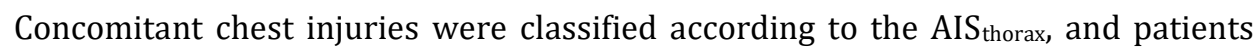
were considered as having a concomitant chest injury when the $\mathrm{AIS}_{\text {thorax }}$ was $\geq 2$.

\subsubsection{Classification of TBI}

TBIs were prehospitally classified according to the Glasgow coma scale (GCS) ${ }^{16}$ and after computer tomography scanning. TBI was additionally classified according to the AIS head. Patients were only considered as having a concomitant TBI when they had a prehospital GCS $\leq 12$ and an AIShead $\geq 2$.

\subsubsection{Fracture classification and fracture healing assessment}

Only patients with diaphyseal femoral (A032.A-C) or tibial (A042.A-C) fractures according to the AO (Arbeitsgemeinschaft für Osteosynthesefragen) classification system, were included in the analysis. It was registered if these fractures were open (grade I or II according to Gustilo \& Anderson) or closed.

Radiological imaging was reviewed and evaluated by two independent observers (HA and PK), who were blinded to concomitant injuries. A fracture was considered to be consolidated when both observers determined that three out of four cortices were bridged by a callus. Further, callus formation was quantified according to the fracture healing response described by Spencer ${ }^{17}$. 
Table 3.1. Clinical and radiological parameters.

\begin{tabular}{|c|c|c|c|}
\hline & $\begin{array}{c}\text { Control group } \\
(\mathrm{n}=181)\end{array}$ & $\begin{array}{c}\text { NU group } \\
(n=23)\end{array}$ & $p$ value \\
\hline \multicolumn{4}{|l|}{ Clinical parameters } \\
\hline \multicolumn{4}{|l|}{ General } \\
\hline Age (years) & $35.5 \pm 15.5$ & $39.7 \pm 15.1$ & 0.167 \\
\hline Gender (female to male ratio) & 0.42 & 0.33 & 0.652 \\
\hline \multicolumn{4}{|l|}{ General health status } \\
\hline ASA classification system & $1.2 \pm 0.7$ & $1.3 \pm 0.7$ & 0.489 \\
\hline CCI & $0.6 \pm 1.3$ & $0.8 \pm 1.2$ & 0.334 \\
\hline \multicolumn{4}{|l|}{ Injury severity } \\
\hline ISS & $11.0 \pm 8.8$ & $10.7 \pm 7.5$ & 0.869 \\
\hline GCS & $14.2 \pm 2.4$ & $14.4 \pm 2.3$ & 0.622 \\
\hline AIS head & $0.4 \pm 0.9$ & $0.3 \pm 1.0$ & 0.753 \\
\hline AIS thorax & $0.4 \pm 1.0$ & $0.3 \pm 1.0$ & 0.735 \\
\hline Concomitant injuries & $54.2 \%$ & $50.0 \%$ & 0.699 \\
\hline & A $55.3 \%$ & A $45.8 \%$ & \\
\hline AO classification & В $36.3 \%$ & В $37.5 \%$ & 0.385 \\
\hline & C $8.4 \%$ & C $16.7 \%$ & \\
\hline Open/closed fracture & $\begin{array}{c}\text { Closed } 80.6 \% \\
\text { Open } 19.4 \% \\
\end{array}$ & $\begin{array}{c}\text { Closed } 66.7 \% \\
\text { Open } 33.3 \% \\
\end{array}$ & 0.117 \\
\hline \multicolumn{4}{|l|}{ Clinical course } \\
\hline Duration of hospital stay (days) & $17.1 \pm 17.6$ & $19.8 \pm 14.0$ & 0.535 \\
\hline In-hospital complications ** & $23.3 \%$ & $20.0 \%$ & 0.785 \\
\hline Period trauma: first operative treatment (days) & $0.4 \pm 1.1$ & $0.6 \pm 1.9$ & 0.506 \\
\hline Period trauma-definitive osteosynthesis (days) & $3.9 \pm 5.3$ & $11.5 \pm 38.2$ & $0.012^{*}$ \\
\hline Period trauma-consolidation (days) & $326.5 \pm 278.3$ & - & \\
\hline Period definitive osteosynthesis-discharge & $15.9 \pm 17.4$ & $17.5 \pm 14.0$ & 0.663 \\
\hline Period definitive osteosynthesis-consolidation (days) & $322.2 \pm 277.5$ & - & \\
\hline Period trauma-discharge & $17.7 \pm 17.6$ & $19.8 \pm 14.0$ & 0.574 \\
\hline Total number of operations performed & $1.5 \pm 0.5$ & $2.1 \pm 0.8$ & $<0.001 *$ \\
\hline ETC vs. DCO & $\begin{array}{l}\text { ETC } 51.1 \% \\
\text { DCO } 48.9 \% \\
\end{array}$ & $\begin{array}{l}\text { ETC } 25.0 \% \\
\text { DCO } 75.0 \% \\
\end{array}$ & $0.016^{*}$ \\
\hline \multicolumn{4}{|l|}{ Radiological parameter } \\
\hline Fracture Healing Response & $1.5 \pm 0.3$ & $0.4 \pm 0.7$ & $<0.001^{*}$ \\
\hline Consolidation ( 3 out of 4 cortices) & $100 \%$ & $0 \%$ & \\
\hline
\end{tabular}

* Statistical significance, $\mathrm{p}<0.05$

** Any secondary neurological, cardiopulmonary, vascular, urinary tract, orthopaedic, and other complications were registered.

\subsubsection{Outcome \& complications}

In addition to our primary outcome parameter of non-union, further neurological, cardiopulmonary, vascular, urinary tract, orthopaedic, and systemic complications were registered. 


\subsubsection{Statistical methods}

Data were analysed using SPSS (version 25; IBM Inc., Somers, NY, USA). Incidences are presented with counts and percentages, while continuous values are presented as mean \pm standard deviation. Differences between the groups were evaluated with Mann-Whitney's $U$ test for continuous data, and Pearson's $\chi^{2}$ test was used for categorical values. The nonparametric Spearman rank test was used for statistical correlation. Multivariate logistic regression analysis was performed with non-union as the dependent variable to adjust for confounding variables. The results were reported as odds ratio with $95 \%$ confidence intervals (95\% CI). In general, a twosided $\mathrm{p}$ value $<0.05$ was considered to be significant.

\subsection{RESULTS}

\subsubsection{Demographic data}

A total of 136 and 68 patients were treated at the University Hospital RWTH Aachen (Germany) and the Maastricht University Medical Centre (The Netherlands), respectively. Of these, 100 patients (49.0\%) had femoral fractures and $104(51.0 \%)$ had tibial fractures. Overall, $25(12.3 \%)$ patients had a concomitant chest injury and $27(13.2 \%)$ had a concomitant TBI. A total of 98 patients $(48.0 \%)$ underwent ETC, and $106(52.0 \%)$ underwent DCO. Conversion to definitive osteosynthesis was performed $6.2 \pm 5.7$ days after trauma (Table 3.1.).

\subsubsection{General health status, injury severity, and clinical course}

The general health status and the injury severity, distribution, and characteristics did not significantly differ between the two study groups. Over the clinical course, significant differences for the time period until definitive fracture stabilization $(\mathrm{p}=$ 0.012 ), the total number of operations performed before consolidation $(p<0.001)$, and the ratio of ETC to DCO (p=0.016) were observed between the control and NU groups (Table 3.1.).

\subsubsection{Nonparametric correlation analysis referring to non-union}

Non-union was diagnosed in $11.3 \%(n=23)$ of our patient population. Nonparametric correlation analysis showed a correlation between the fracture healing response and non-union development $(r=-0.424, p<0.001)$. Also, the CCI was correlated with the incidence of non-union $(r=0.148, p=0.034)$. For TBI and chest trauma, no correlation was found. (Table 3.2.)

\subsubsection{Multivariate regression analysis referring to non-union}

The multivariate regression analysis referring to non-union showed that only the total number of operations before consolidation was an independent risk factor for non-union development (OR $=6.416$; $\mathrm{p}<0.001$; Table 3.3.).

Most patients underwent one or two operations and only seven patients underwent more than two operations, which were performed due to hardware failure $(n=3)$ or infection $(n=4)$, and not because of disturbed healing. Therefore, we focused on the patients who underwent either one or two operations. Of these patients, 96 underwent ETC and 86 of the 101 patients who had 2 operations (85.1\%) underwent DCO. In the multivariate regression analysis referring to non-union in DCO vs. ETC, 
DCO represented an independent risk factor for non-union development with an odds ratio of $7.878(\mathrm{p}=0.005$; Table 3.4.).

Table 3.2. Non-parametric correlation analysis of DCO treatment.

\begin{tabular}{lcc}
\hline Parameter & Correlation coefficient (r) & $p$ value \\
\hline Age & -0.277 & $<0.001^{* *}$ \\
Gender & -0.208 & $0.005^{* *}$ \\
ASA & 0.162 & $0.028^{*}$ \\
ISS & 0.471 & $<0.001^{* *}$ \\
AIS head & 0.282 & $<0.001^{* *}$ \\
AIS thorax & 0.308 & $<0.001^{* *}$ \\
Concomitant injuries & 0.449 & $<0.001^{* *}$ \\
Complications & 0.314 & $<0.001^{* *}$ \\
AIS extremity & 0.236 & $0.001^{* *}$ \\
Open / closed fractures & -0.017 & 0.815 \\
Duration of hospital stay (days) & 0.729 & $<0.001^{* *}$ \\
Non-union & 0.161 & $0.029^{*}$ \\
\hline
\end{tabular}

* Statistical significance $\mathrm{p}<0.05$

** Statistical significance $\mathrm{p}<0.01$

\subsection{DISCUSSION}

Non-unions of long bone fractures represent a challenging problem in trauma patients. Patient-, injury- and treatment-specific factors have been previously described to influence the occurrence of non-unions. Independent from the already well-known risk factors for non-union development, we aimed to focus on the impact of chest injury, TBI, and the fracture stabilisation strategy on the occurrence of nonunions in diaphyseal long bone fractures. Our main results can be summarised as follows:

1. The DCO fracture stabilisation strategy represents an independent risk factor for the development of non-unions in long bone fractures.

2. Chest injury and TBI were not identified as influencing factors for non-union development in diaphyseal long bone fractures.

Although DCO treatment is well accepted to be beneficial in certain subgroups of trauma patients, we found that this treatment strategy is associated with a higher risk of non-union. Our findings were in accordance to those reported in a previous study by Rixen et al. ${ }^{18}$. In particular, the timing of conversion from external fixation to definitive stabilization has been suggested as an indispensable factor for nonunion development ${ }^{19,20}$. In this context, late conversion ( $>10$ days after the initial treatment) has been associated with an increase of fracture-associated complications, such as non-union ${ }^{21}$. Therefore, it is of utmost importance to plan definitive surgery meticulously. In this context, Pape and Pfeifer revitalized the 
discussion on the DCO treatment strategy by introducing the concept of safe definitive surgery (SDS). In this concept, the time point of definitive fracture stabilization is based on a regular re-evaluation and assessment of the patient's physiological condition and not on a suggested time point like in the DCO concept (e.g. not before day 5). The SDS concept therefore might lead to a dynamic combination of the advantages of both the DCO and ETC treatment strategy ${ }^{22}$. Our findings support the philosophy of this approach. Furthermore, approaches to identifying patients who could potentially benefit from DCO should be improved to avoid the increased risk of non-union development. To assess the relevance of concomitant injuries, we focused on chest injuries and TBI.

Table 3.3. Multivariate regression analysis referring to non-union analysing age, gender, ASA, CCI, ISS, GCS, AIS head, $_{\text {AIS }}$ thorax, concomitant injuries, AO-classification, open/closed fracture, period between trauma and definitive osteosynthesis, and the total number of operations before consolidation as potential predictors (Nagelkerke: $\mathrm{R}^{2}=0.294$ ).

\begin{tabular}{|c|c|c|c|c|}
\hline Predictor & $\begin{array}{l}\text { Regression } \\
\text { coefficient }\end{array}$ & $\begin{array}{c}\text { Odds ratio } \\
\text { (OR) }\end{array}$ & $\begin{array}{l}95 \% \text { confidence interval } \\
(95 \%-\mathrm{CI}) \\
\end{array}$ & $p$ value \\
\hline \multicolumn{5}{|l|}{ Patient-specific } \\
\hline Age (years) & 0.039 & 1.040 & 0.995-1.086 & 0.083 \\
\hline Gender (male) & 0.203 & 1.225 & $0.358-4.186$ & 0.747 \\
\hline ASA & -0.070 & 0.933 & $0.404-2.152$ & 0.870 \\
\hline CCI & 0.017 & 1.017 & $0.616-1.679$ & 0.947 \\
\hline \multicolumn{5}{|l|}{ Injury-specific } \\
\hline ISS & -0.022 & 0.978 & $0.895-1.069$ & 0.624 \\
\hline GCS & 0.513 & 1.670 & $0.638-4.369$ & 0.296 \\
\hline $\mathrm{AIS}_{\text {head }}$ & 0.579 & 1.784 & $0.462-6.897$ & 0.401 \\
\hline $\mathrm{AIS}_{\text {thorax }}$ & -0.066 & 0.936 & $0.489-1.792$ & 0.842 \\
\hline Concomitant injuries & -0.236 & 0.790 & $0.235-2.661$ & 0.704 \\
\hline AO-classification & 0.630 & 1.878 & $0.355-9.954$ & 0.459 \\
\hline Open/closed fracture & 0.651 & 1.917 & $0.571-6.440$ & 0.292 \\
\hline \multicolumn{5}{|l|}{ Treatment-specific } \\
\hline Period trauma-definitive osteosynthesis (days) & 0.042 & 1.043 & $0.995-1.094$ & 0.077 \\
\hline Total number of operations before consolidation & 1.859 & 6.416 & $2.434-16.910$ & $<0.001 *$ \\
\hline Constant & -14.309 & & & 0.058 \\
\hline
\end{tabular}

* Statistical significance $\mathrm{p}<0.05$

However, both entities did not significantly influence the development of nonunions. In contrast to our study, Recknagel et al. suggest that chest trauma has a negative effect, particularly on the late phases of bone regeneration and fracture healing ${ }^{23}$. A chest trauma-associated hypoxemia-induced enhancement of local and systemic inflammation has been suggested as a potential pathomechanism by Kemmler et al. ${ }^{24}$. The differences between the results of our study and these experimental studies might be explained with different aspects. First, data obtained in animal experiments under standardized conditions might not be point-to-point transferable to the clinical situation with different confounding factors. Second, it has been postulated that the strategy for fracture fixation is an even more important factor for fracture healing than concomitant injuries ${ }^{23}$. This would be in line with our 
results and might explain why we did not observe an impact of chest trauma on the incidence of non-unions.

TBI did not have a significant influence on fracture healing. Therefore, the findings of this study are in contrast to the findings of the majority of studies that TBI has a positive influence on bone regeneration ${ }^{11}$. In this context, a retrospective study 8 found shorter healing time and increased callus dimensions in patients with concomitant TBI. In contrast to our study, they excluded patients treated according to the DCO principle. As fracture fixation represented an independent risk factor for disturbed fracture healing in our study, this might be one explanation for the different results of the studies. This assumption would also support the finding of the aforementioned experimental study that fracture fixation has more impact on fracture healing than concomitant injuries ${ }^{23}$. Another clinical study demonstrated shorter healing times, greater callus volumes, and higher fracture healing rates in patients with concomitant TBI ${ }^{25}$. Contrary to our study, they included all long bones fractures (including humeral and fibular fractures) treated either with intramedullary nailing or plate osteosynthesis. Furthermore, they included only patients with severe TBI $(\mathrm{GCS}<8)$. These differences are likely to contribute to the differences between that study and our present study.

Table 3.4. Multivariate regression analysis referring to non-union analysing age, gender, ASA, CCI, ISS, GCS, AIS head, $_{\text {AIS }}$ thorax, concomitant injuries, AO-classification, open/closed fracture, period between trauma and definitive osteosynthesis, and DCO vs. ETC as potential predictors (Nagelkerke: $\mathrm{R}^{2}=0.215$ ).

\begin{tabular}{|c|c|c|c|c|}
\hline Predictor & $\begin{array}{l}\text { Regression } \\
\text { coefficient }\end{array}$ & $\begin{array}{c}\text { Odds ratio } \\
\text { (OR) }\end{array}$ & $\begin{array}{l}95 \% \text { confidence interval } \\
(95 \%-\mathrm{CI})\end{array}$ & $p$ value \\
\hline \multicolumn{5}{|l|}{ Patient-specific } \\
\hline Age (years) & 0.036 & 1.037 & $0.990-1.086$ & 0.123 \\
\hline Gender (male) & 0.406 & 1.501 & $0.424-5.313$ & 0.529 \\
\hline ASA & -0.183 & 0.833 & $0.348-1.993$ & 0.681 \\
\hline $\mathrm{CCI}$ & -0.007 & 0.993 & $0.568-1.736$ & 0.980 \\
\hline \multicolumn{5}{|l|}{ Injury-specific } \\
\hline ISS & -0.020 & 0.980 & $0.900-1.067$ & 0.646 \\
\hline GCS & 0.467 & 1.596 & $0.640-3.977$ & 0.316 \\
\hline $\mathrm{AIS}_{\text {head }}$ & 0.500 & 1.648 & $0.446-6.094$ & 0.454 \\
\hline $\mathrm{AIS}_{\text {thorax }}$ & -0.069 & 0.934 & $0.493-1.769$ & 0.833 \\
\hline Concomitant injuries & -0.374 & 0.688 & $0.185-2.565$ & 0.578 \\
\hline AO-classification & 0.680 & 1.974 & $0.368-10.583$ & 0.427 \\
\hline Open/closed fracture & 0.714 & 2.042 & $0.577-7.219$ & 0.268 \\
\hline \multicolumn{5}{|l|}{ Treatment-specific } \\
\hline Period trauma-definitive osteosynthesis (days) & 0.039 & 1.040 & $0.994-1.088$ & 0.088 \\
\hline DCO vs. ETC & 2.064 & 7.878 & $1.889-32.860$ & $0.005^{*}$ \\
\hline Constant & -13.780 & & & 0.056 \\
\hline
\end{tabular}

* Statistical significance $\mathrm{p}<0.05$ 


\subsubsection{Strength and limitations}

A strength of our study design is that by strict inclusion and exclusion criteria, we were able to analyse a specific patient cohort with smaller parameter variance and better comparability, in which we could focus on the influence of chest injury, TBI, and fracture stabilisation strategy on non-union development by eliminating other possible confounding factors as much as possible.

One limitation of this study is its retrospective design. Second, a large number of patients (114) were lost to follow up. Some of those patients may have had complications from the treatment and went for care elsewhere. On the other hand, patients with a straightforward healing process may have disengaged from the follow-up because they did not think it was essential. These phenomena could lead to possible selection bias.

Third, the treatment evaluated was limited to reamed intramedullary nailing and in consequence we cannot assess the influence of the studied parameter on non-unions in diaphyseal long bone fractures following other treatment strategies. However, reamed intramedullary nailing is an established technique and is the preferred therapy for long bone shaft fractures of the lower extremities in adults ${ }^{5}$.

\subsection{CONCLUSION}

Our results demonstrated that fracture stabilisation strategy is a far more powerful factor than concomitant injuries influencing non-union development in long bone fractures. Based on our finding that DCO stabilisation strategy is an eminent predictor for non-union development, it is of utmost importance in the clinical situation to critically review both: the indication for DCO and the time period until conversion to definitive treatment to minimize the risk of disturbed fracture healing. Our study further counterweights the rising evidence of concomitant chest injury predisposing and concomitant TBI protecting for non-unions in the specific situation of diaphyseal long bone fractures of the lower extremities. These findings could contribute to the improvement of the treatment principles and to the reduction of the treatment costs of non-union and its sequelae. Furthermore, reliably predicting the risk of non-union in certain fractures at the time of initial treatment would be a great advantage and could possibly modify treatment management.

\subsection{ACKNOWLEDGEMENTS}

Conflict of interest. All authors declare that they have no conflict of interest.

Ethical approval. All data in this study were obtained in accordance with the ethical standards of both institutional and/or national research committee and the guidelines of the revised United Nations declaration of Helsinki in 2013 (seventh revision) or comparable ethical standards.

\subsection{REFERENCES}

1. Giannoudis PV, Einhorn TA, Marsh D. Fracture healing: the diamond concept. Injury 2007; 38 Suppl 4: S3-6.

2. Tay WH, de Steiger R, Richardson M, Gruen R, Balogh ZJ. Health outcomes of delayed union and

nonunion of femoral and tibial shaft fractures. Injury 2014; 45(10): 1653-8.

3. Tzioupis C, Giannoudis PV. Prevalence of longbone non-unions. Injury 2007; 38 Suppl 2: S3-9. 
4. Giannoudis PV, Jones E, Einhorn TA. Fracture healing and bone repair. Injury 2011; 42(6): 549-50.

5. Metsemakers WJ, Roels N, Belmans A, Reynders P, Nijs S. Risk factors for nonunion after intramedullary nailing of femoral shaft fractures: Remaining controversies. Injury 2015; 46(8): 1601-7.

6. Hildebrand F, van Griensven M, Huber-Lang M, et al. Is There an Impact of Concomitant Injuries and Timing of Fixation of Major Fractures on Fracture Healing? A Focused Review of Clinical and Experimental Evidence. Journal of orthopaedic trauma 2016; 30(3): 104-12.

7. Cadosch D, Gautschi OP, Thyer M, et al. Humoral factors enhance fracture-healing and callus formation in patients with traumatic brain injury. The Journal of bone and joint surgery American volume 2009; 91(2): 282-8.

8. Giannoudis PV, Mushtaq S, Harwood P, et al. Accelerated bone healing and excessive callus formation in patients with femoral fracture and head injury. Injury 2006; 37 Suppl 3: S18-24.

9. Yang TY, Wang TC, Tsai YH, Huang KC. The effects of an injury to the brain on bone healing and callus formation in young adults with fractures of the femoral shaft. The Journal of bone and joint surgery British volume 2012; 94(2): 227-30.

10. Morley J, Marsh S, Drakoulakis E, Pape HC, Giannoudis PV. Does traumatic brain injury result in accelerated fracture healing? Injury 2005; 36(3): 3638.

11. Hofman M, Koopmans G, Kobbe P, et al. Improved fracture healing in patients with concomitant traumatic brain injury: proven or not? Mediators of inflammation 2015; 2015: 204842.

12. Song Y, Han GX, Chen L, et al. The role of the hippocampus and the function of calcitonin generelated peptide in the mechanism of traumatic brain injury accelerating fracture-healing. European review for medical and pharmacological sciences 2017; 21(7): 1522-31.

13. Wildburger R, Zarkovic N, Tonkovic G, et al. Posttraumatic hormonal disturbances: prolactin as a link between head injury and enhanced osteogenesis. Journal of endocrinological investigation 1998; 21(2): 78-86.

14. Charlson ME, Pompei P, Ales KL, MacKenzie CR. A new method of classifying prognostic comorbidity in longitudinal studies: development and validation. Journal of chronic diseases 1987; 40(5): 373-83.

15. Baker SP, O'Neill B, Haddon W, Jr., Long WB. The injury severity score: a method for describing patients with multiple injuries and evaluating emergency care. The Journal of trauma 1974; 14(3): 187-96.

16. Teasdale G, Jennett B. Assessment of coma and impaired consciousness. A practical scale. Lancet 1974; 2(7872): 81-4.

17. Spencer RF. The effect of head injury on fracture healing. A quantitative assessment. The Journal of bone and joint surgery British volume 1987; 69(4): 525-8.

18. Rixen D, Grass G, Sauerland S, et al. Evaluation of criteria for temporary external fixation in riskadapted damage control orthopedic surgery of femur shaft fractures in multiple trauma patients: "evidencebased medicine" versus "reality" in the trauma registry of the German Trauma Society. The Journal of trauma 2005; 59(6): 1375-94; discussion 94-5.

19. Pape H, Stalp M, v Griensven M, Weinberg A, Dahlweit M, Tscherne H. [Optimal timing for secondary surgery in polytrauma patients: an evaluation of 4,314 serious-injury cases]. Der Chirurg; Zeitschrift fur alle Gebiete der operativen Medizen 1999; 70(11): 1287-93.

20. Schuh A, Hausel M. [Our experiences with change of osteosynthesis from external fixator to internal fixation in fractures of the lower limb]. Zentralblatt fur Chirurgie 2003; 128(8): 674-9.

21. Antich-Adrover P, Marti-Garin D, Murias-Alvarez J, Puente-Alonso C. External fixation and secondary intramedullary nailing of open tibial fractures. A randomised, prospective trial. The Journal of bone and joint surgery British volume 1997; 79(3): 433-7.

22. Pape HC, Pfeifer R. Safe definitive orthopaedic surgery (SDS): repeated assessment for tapered application of Early Definitive Care and Damage Control?: an inclusive view of recent advances in polytrauma management. Injury 2015; 46(1): 1-3.

23. Recknagel S, Bindl R, Kurz J, et al. Experimental blunt chest trauma impairs fracture healing in rats. Journal of orthopaedic research : official publication of the Orthopaedic Research Society 2011; 29(5): 734-9.

24. Kemmler J, Bindl R, McCook O, et al. Exposure to $100 \%$ Oxygen Abolishes the Impairment of Fracture Healing after Thoracic Trauma. PloS one 2015; 10(7): e0131194.

25. Khallaf FG, Kehinde EO, Hussein S. Bone Healing and Hormonal Bioassay in Patients with Long-Bone Fractures and Concomitant Head Injury. Medical principles and practice : international journal of the Kuwait University, Health Science Centre 2016; 25(4): 336-42. 


\title{
Effect of neurokinin-1-receptor blockage on fracture healing in rats.
}

\section{Scientific Reports}

2019 Jul 5;9(1):9744. doi: 10.1038/s41598-019-46278-6. PMID:

31278316, PMCID: PMC6611911.

\author{
Martijn Hofman ${ }^{1}$ \\ Frederik Rabenschlag ${ }^{1}$ \\ Hagen Andruszkow ${ }^{1}$ \\ Julia Andruszkow ${ }^{2}$ \\ Diana Möckel ${ }^{3}$ \\ Twan Lammers ${ }^{3}$ \\ Aneta Kolejewska ${ }^{1}$ \\ Philipp Kobbe ${ }^{1}$ \\ Johannes Greven ${ }^{1}$ \\ Michel P.J. Teuben ${ }^{4,5}$ \\ Martijn Poeze ${ }^{6 \dagger}$ \\ Frank Hildebrand ${ }^{1 \dagger}$
}

1 Department of Orthopaedic Trauma and Reconstructive Surgery, University Medical Center RWTH Aachen, Germany.

2 Institute of Pathology, University Medical Center RWTH Aachen, Germany.

3 Institute for Experimental Molecular Imaging, Center for Biohybrid Medical Systems, University Medical Center RWTH Aachen, Germany.

4 Department of Trauma, University Hospital Zürich, Switzerland.

5 Harald Tscherne Laboratory, University Hospital Zürich, Switzerland.

6 Department of Trauma Surgery, Maastricht University Medical Center +, the Netherlands.

$\dagger$ Authors contributed mutually 


\section{ABSTRACT}

Neurologic injury and selective blockage of sensory nerve endings is associated with impaired fracture healing, however, the role of specific neurotransmitters has not been sufficiently investigated. Our aim was to investigate the impact of specific Substance P-receptor blockage on fracture healing, since the neuropeptide Substance $\mathrm{P}$ has both neurogenic and osteogenic activity. After intramedullary stabilization, an isolated femur fracture was induced in 72 Sprague-Dawley rats. In the NK1-R group, the neurokinin-1-tachykinin receptor for substance P was blocked by a specific antagonist (SR140333) for the first two weeks after fracture induction. The control group only received vehicle. Histology, micro-computed tomography, biomechanical tests, and gene-expression analysis were performed. NK1-receptor blocking suppressed osteocalcin expression at one week, collagen 1A2 expression at one and two weeks and collagen 2A1 expression at 2 weeks after fracture induction. Biomechanical testing revealed a significant reduction in maximal load to failure in the NK1-R group at 6 weeks (69.78 vs. $155.45 \mathrm{~N}, \mathrm{p}=0.029$ ) and at 3 months (72.50 vs.176.33 N, p = 0.01) of fracture healing. Blocking the NK1-receptor suppresses gene expression in and reduces biomechanical strength of healing bone. Therefore, we assume a potential therapeutic relevance of Substance $\mathrm{P}$ in cases of disturbed fracture healing. 


\subsection{INTRODUCTION}

Fractures are frequently associated with either central (e.g., traumatic brain injury [TBI]) or peripheral neurological injuries, and these injuries are well known to affect fracture healing. This phenomenon has partly been explained by the close anatomical relationship between bones and nerves (e.g., innervation of periosteum with close contact between nerve endings and bone cells). It also reflects the chronological association between callus formation, bone remodelling, and the regeneration of damaged nerve endings and ingrowth into newly formed bone ${ }^{1,2}$. Disturbances of this re-innervation have therefore been associated with the incidence of non-unions 3. Hukkanen and colleagues provided the first evidence for a role of nerve-mediated bone formation in fracture healing by showing that a complete denervation of a leg by sciatic nerve section had negative effects on the mechanical integrity of the bony callus after fracture ${ }^{4}$. Similarly, Offley and colleagues found that capsaicin-sensitive neurons contribute to cancellous bone integrity and bone homeostasis in the uninjured bone. They concluded that osteoclast numbers, osteoblast activity, bone formation, and bone strength were mediated by transmitters released from efferent sensory nerve endings in bone tissue ${ }^{5}$.

Further, complete peripheral nerve transection ${ }^{6-8}$ and selective blockade of sensory nerve endings ${ }^{9}$ can impair fracture healing, as demonstrated by previous studies utilizing either combined motoric, sensory, and autonomic denervation 7,8 or complete sensory nerve ending blockade ${ }^{5,9}$.

The exact pathophysiologic mechanism underlying this interaction between fracture healing and nerve injuries is not known. Neuropeptides are produced by nerve endings after TBI and fractures, so neuropeptides potentially have major relevance in the process of fracture healing 1,2,10,11. However, no precise role has been established for specific neuropeptides in bone healing ${ }^{12,13}$.

One neuropeptide of particular relevance to bone healing is Substance $P$, which shows simultaneous effects at the neurogenic and osteogenic activities. For example, Apel et al. showed that sensory denervation, which shut down the transmission of both calcitonin gene-related peptide (CGRP) and Substance P, resulted in an impaired upregulation of collagen I and II in fracture callus and a negative influence on osteoclast function and mechanical strength and maturation of fracture callus. ${ }^{9}$ Neurotransmitters were therefore postulated to play a central role in the interaction between the nerves and the osseous system ${ }^{8 ; 31}$, and especially substance $\mathrm{P}$ seems to have a significant involvement in bone metabolism, formation, and resorption, as well as in the osteogenic activity of bone marrow stromal cells and osteogenic cell lines ${ }^{14-22}$. However, the relevance of specific neurotransmitters remains unclear in these previous studies and the specific effects of Substance P on the process of fracture healing have not been elucidated. Therefore, the aim of this study was to investigate the effects of specific Substance P-receptor blockage on gene expression, callus formation, and the mechanical strength of healing fractures in an isolated femur fracture model in rats. We hypothesize that specific substance $\mathrm{P}$ receptor blockage impairs mechanical strength of healing fractures, decreases geneexpression in the early fracture healing stages and reduces callus quantity and quality. 


\subsection{METHODS}

All methods were performed with the approval of the institutional animal committee and of the regulating authority (LANUV) North Rhine-Westphalia, Recklinghausen, Germany (AZ 84-02.04.2015.A078). All animal experiments were performed in accordance with the guidelines and regulations of the Federation of European Laboratory Animal Science Associations (FELASA) and the German Society of Laboratory Animals (GV-SOLAS).

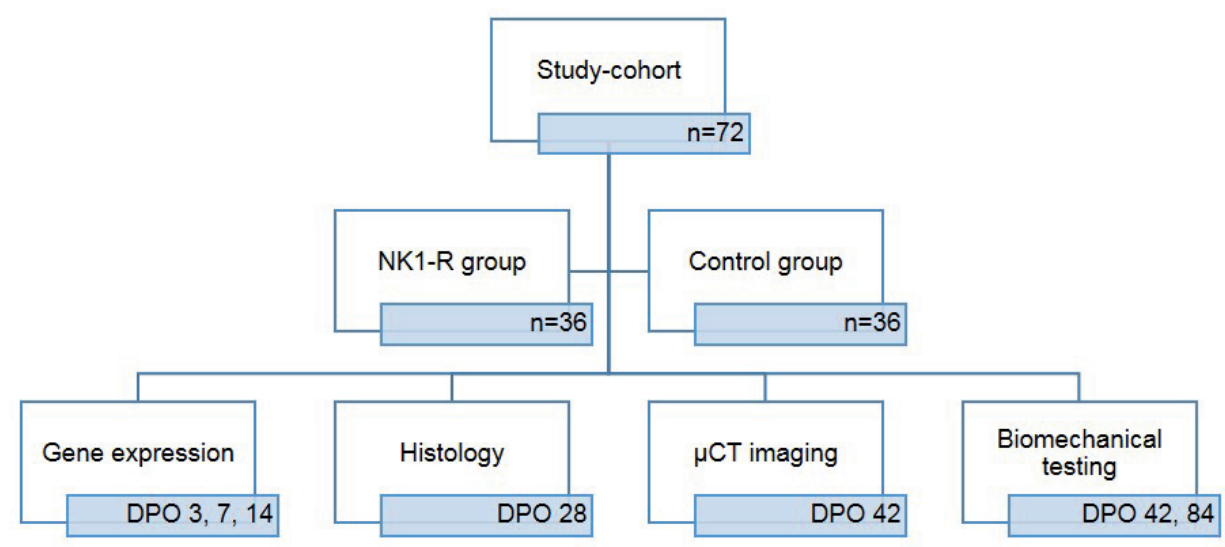

Figure 4.1. Subgroup division and time points of analyses. Subdivision of study cohort in two study groups (NK1-R group and Control group) and subsequent division of animals according to performed analysis at different time points. Every analysis is performed in 6 animals of both study groups at every time point. At DPO (days post-operative) 42 both $\mu$ CT imaging and biomechanical testing was performed in the same animals.

\subsubsection{Housing}

Our study cohort consisted of 72 adult, female Sprague-Dawley rats, weighing approximately $250 \mathrm{~g}$, obtained from Harlan Industries (Indianapolis, Indiana, USA). The animals were housed and the experiments were performed in facility approved by the Federation of European Laboratory Animal Science Associations (FELASA) and the German Society of Laboratory Animals (GV-SOLAS). The animals were housed under conditions of controlled temperature $\left(20 \pm 2{ }^{\circ} \mathrm{C}\right)$ and air humidity (45$65 \%$ ), with a $12 \mathrm{~h}$ light-darkness cycle and a light intensity of $<200$ lux. Food and water were offered ad libitum. Prior to study inclusion, all animals were kept in groups for one week in the laboratory premises to allow acclimatization. Throughout the entire experiment, all animals underwent physical examinations according to a 'score sheet' documentation and the 'Body condition scoring' according to Hickman 23 to obtain the general health of the animals.

Because of the protective effects of female hormones in cases of inflammatory stimuli, all animals were confirmed to be in the same 'metestrus' phase of the menstrual cycle, as this phase is characterized by low oestrogen and progesterone 
levels 24,25 . The menstrual cycle phase was identified by the assessment of vaginal swabs according to Marcondes and colleagues ${ }^{26}$.

\subsubsection{Experimental Design and Power Analysis}

In all animals an intramedullary pin was inserted and a standardized femoral fracture was induced. The animals were then randomly assigned to 2 groups (36 rats/group), according to their order number (odd or even). The first group underwent a selective blockage of the NK1-receptor (NK1-R group). The second group served as the control group and only received vehicle (Control group). Depending on the experimental subgroup to which an animal was randomly assigned, the femur was harvested and the following analyses were executed at different time points: gene expression analysis, histological analyses, micro-CT scanning, and biomechanical testing. (Figure 4.1.)

Our primary outcome parameter was the load to failure of the rat femora; therefore, our power analysis was based on an average load to failure of $131.3 \pm 4.9 \mathrm{~N}$ for normal rat femora, as described by previous studies 9,27 . Our calculation indicated that a minimum of 4 rats per group was needed to achieve a $95 \%$ power for detecting a difference of $10 \mathrm{~N}$ with $\alpha=0.05$. Therefore, a sample size of six animals per group was chosen to compensate for any potential loss of animals. The power analysis was performed with $G^{*}$ Power. Secondary outcome parameters were fold change in gene expression of osteocalcin and collagen $1 \mathrm{~A} 2$ and 2A1 and callus-volume, -density and -diameter in micro-CT-analysis.

Reasons for excluding animals were: death from anaesthetic complications, open fracture, comminuted fracture, implant failure, wound dehiscence or infection, gross technical failure, inadequate RNA for analysis, and unintended displacement of the femur during biomechanical testing.

\subsubsection{Anaesthesia and Pain Management}

Thirty minutes preoperatively, the animals received $0.03-0.05 \mathrm{mg} / \mathrm{kg}$ buprenorphine hydrochloride s.c. as pain medication. The operative procedures were performed under general anaesthesia induced with ketamine (100 mg/kg i.p.) and xylazine (2\%; $10 \mathrm{mg} / \mathrm{kg}$ i.p.) and if necessary, extended with 2-2.5 Vol.\% isoflurane inhalation. The toe pinch reflex was used to assure adequate anaesthesia. Post-operative analgesia was assured with buprenorphine hydrochloride (0.03-0.05 $\mathrm{mg} / \mathrm{kg}$ s.c.) every $6 \mathrm{~h}$ for the first 24-48 h. Subsequently, buprenorphine hydrochloride was given twice daily during the first 3 weeks. Furthermore, in the first postoperative week, the drinking water was supplemented with metamizole (1 $\mathrm{ml} / 300 \mathrm{ml}$ ). The animals were evaluated postoperatively three times per day for signs of acute deterioration.

\subsubsection{Standardized Femoral Fracture}

After anaesthesia induction, the animals were placed on a heated pad $\left(37^{\circ} \mathrm{C}\right)$ and their eyes were covered with moistening ointment. The right rear leg was shaved, disinfected, and draped. Then, a para-patellar incision was made, the patella was everted laterally and a $1.0 \mathrm{~mm}$ stainless-steel intramedullary Kirschner wire was inserted in a retrograde manner. Its placement was confirmed by fluoroscopy. The $\mathrm{K}$-wire was cut flush with the intercondylar notch and the proximal end was bent 
over the greater trochanter, cut, and hidden subcutaneously. The patella was repositioned and the wounds were closed in layers. Fracture induction was performed with a blunt guillotine according to the method of Bonnarens and Einhorn 28. A fluoroscopic evaluation of the fracture site was performed.

\subsubsection{Substance P Receptor Blockage}

Substance P activity was blocked through selective blockage of the neurokinin-1tachykinin-receptor (NK1-R) by subcutaneous administration of SR140333 once daily for the first 14 days, beginning 30 min preoperatively. This period was chosen because it is the most vulnerable period in the fracture healing process in rats in terms of gene expression and the influence of cytokines, chemokines, growth factors, etc. 29,30 . SR140333 is a non-peptide antagonist of tachykinin NK1 receptors that potently, selectively, and competitively inhibits substance $\mathrm{P}$ binding to NK1 receptors ${ }^{31}$. SR140333 was administered at a concentration of $1 \mathrm{mg} / \mathrm{kg}$ dissolved in a vehicle of $10 \mu \mathrm{l}$ DMSO (dimethyl sulfoxide) and $0.2 \mathrm{ml}$ of sterile water ${ }^{32,33}$. The animals of the control group received the same amount of vehicle for the same period.

\subsubsection{Gene Expression}

The up-regulation of most genes associated with fracture healing in rats takes place in the first two weeks after fracture induction 29,30 ; therefore, gene expression was measured at 3,7 , and 14 days post-operative (DPO).

At each time point, 6 animals from each group (NK1-R group and control group) were euthanized with an overdose isoflurane and subsequent cervical dislocation. The intramedullary K-wire was removed and bone and callus at $0.5 \mathrm{~cm}$ on each side of the fracture was harvested, frozen in liquid nitrogen, pulverized, and used for RNA extraction. The fold changes of osteocalcin, collagen 1A2, and collagen 2A1 mRNA expression were determined by the reverse transcription polymerase chain reaction (RT-PCR). The cDNA was transcribed from total RNA using a Maxima H Minus cDNA synthesis kit (Thermo Scientific, US). Real-time quantitative RT-PCR was performed with Power SYBR Green Master Mix (Applied Biosystems, US) using the StepOnePlus ${ }^{\mathrm{TM}}$ Real-Time PCR System (Invitrogen, CA, USA). The $2^{-\Delta \Delta C \mathrm{~T}}$ method (a method to analyse the relative changes in gene expression from real-time qPCR experiments) was used to calculate gene expression with peptidyl prolyl cis-trans isomerase (PPIA) as an internal housekeeping gene reference. The NK1-R and Control groups were compared for their expression of collagen-1A2, collagen-2A1, and osteocalcin mRNA.

\subsubsection{Histological Analysis}

At DPO 28, 10 animals underwent histological analysis ( 6 of the $\alpha$ NK1-R group and 4 of the Control group, due to drop out of 2 animals, see Results section). The animals were euthanized, the Kirschner wire was removed, and the femora were harvested and fixed in $4 \%$ neutral buffered formalin for 48 hours. Decalcification was performed with ethylenediaminetetraacetic acid (EDTA) and dehydration with alcohol. The tissue was embedded in paraffin and $10 \mu \mathrm{m}$ sagittal sections of the callus area and the surrounding soft tissues were cut. The sections were stained with haematoxylin eosin (HE) and assessed with an Olympus BHS System Microscope. 
Images were viewed at $40 \times$ and $200 \times$ magnification, and measurements of the bone were performed.
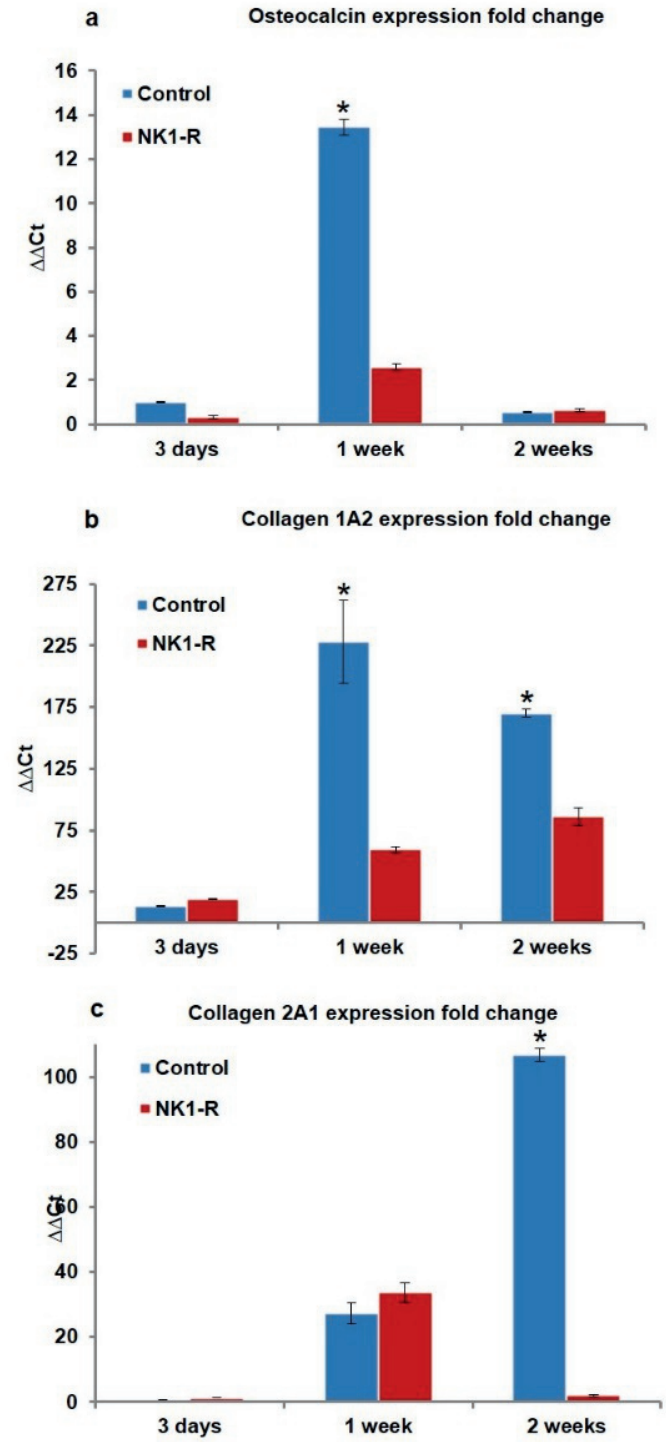

Figure 4.2. Comparison of fold changes in gene expression. The fold changes of osteocalcin (a), collagen $1 \mathrm{~A} 2$ (b), and collagen 2A1 (c) mRNA expression were determined by the reverse transcription polymerase chain reaction (RT-PCR) at 3 days, 1 week, and 2 weeks after fracture induction.

\subsubsection{Micro-Computed Tomography $(\mu \mathrm{CT})$}

Micro-CT imaging was obtained at DPO 42 in 6 euthanized animals from each group using a dual-energy gantry-based flat-panel microcomputed tomography scanner 
(TomoScope 30s Duo, CT Imaging, Erlangen, Germany). The dual-energy X-ray tubes of the $\mu \mathrm{CT}$ were operated at voltages of 40 and $65 \mathrm{kV}$, with currents of 1.0 and 0.5 $\mathrm{mA}$, respectively. Coverage of the entire leg of the rats was achieved by performing three sub-scans; each sub-scan acquired 720 projections with 1,032 $\times 1,012$ pixels during one full rotation, with durations of $90 \mathrm{~s}$. After acquisition, volumetric data sets were reconstructed using a modified Feldkamp algorithm with a smooth kernel at an isotropic voxel size of $35 \mu \mathrm{m}$. The bone, $\mathrm{K}$-wire, and fracture callus regions were segmented using an automated segmentation method with interactive correction of segmentation errors (Software Imalytics Preclinical ${ }^{34}$ ). (Figure 4.4.) The total bone volume, callus volume, bone density, callus density, and transverse diameter of the fracture callus were analysed quantitatively. The mid-part of the femoral shaft of the unfractured side of the animals from the Control group were taken for reference.

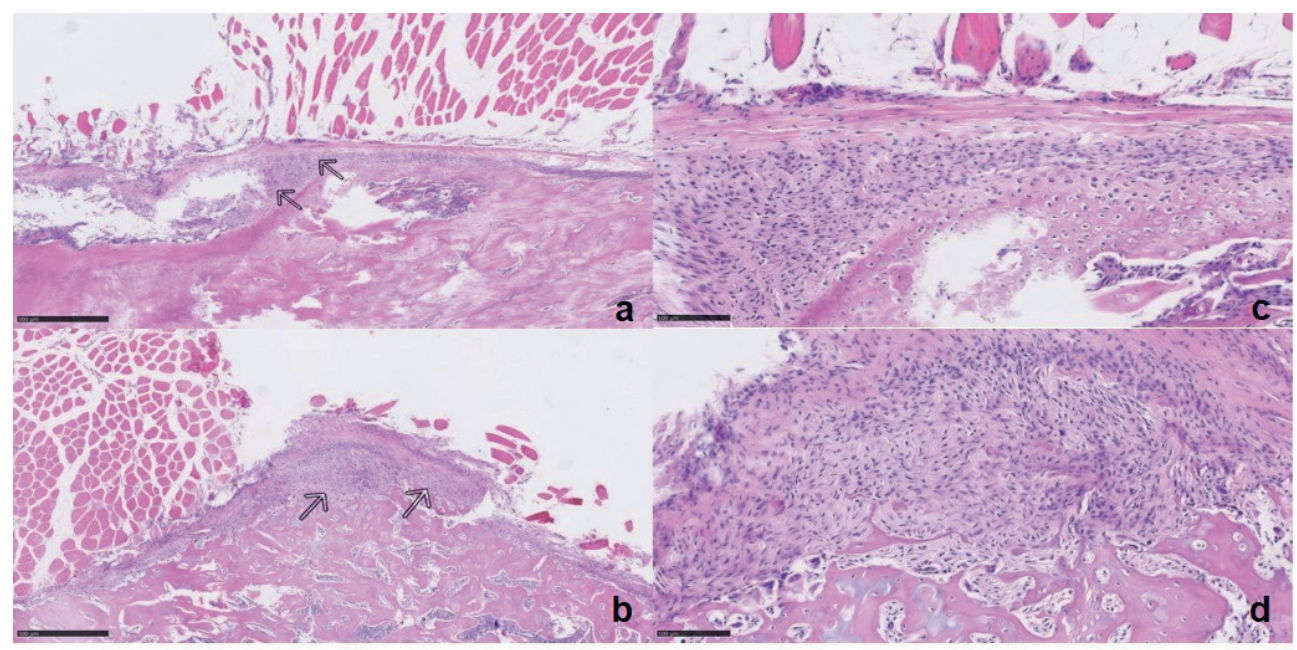

Figure 4.3. Microscopic imaging of callus tissue. Callus tissue (arrows) between muscle cells (above) and bone (below) in control group (a; calibration $=500 \mu \mathrm{m})$ and NK1-R group (b; calibration $=500 \mu \mathrm{m})$ in $5 \times$ magnification using haematoxylin-eosin staining. Callus tissue in control group (c; calibration $=100$ $\mu \mathrm{m})$ and NK1-R group (d; calibration $=100 \mu \mathrm{m})$ in $20 \times$ magnification using haematoxylin-eosin staining.

\subsubsection{Biomechanical Testing}

Biomechanical testing was performed at two time points in the healing process to analyse the strength of the callus and/or newly formed bone. The first test was performed at DPO 42 in 6 animals of both groups; the second test was performed at DPO 84 in 6 animals of both groups. These time points were chosen because normal callus formation is completed after 6 weeks and the remodelling process is advanced or completed after 3 months. The fractured right as well as the unfractured left femur were tested in all animals. After euthanizing the animals and removing the K-wires, both ends of the femora were embedded, closely to the callus / fracture site in a twocomponent resin (Technovit ${ }^{\circledR} 3040$ powder + Technovit ${ }^{\circledR}$ Universal Liquid), ${ }^{35,36}$ which quickly hardens at low temperature. The embedded femora were tested in the biomechanical testing device (Retroline from Zwick Roell AG, Germany), over two cardan yokes, by which the force was conducted perpendicular to the femur axis. The 
biomechanical traction test was performed with a traction rate of $1 \mathrm{~mm} / \mathrm{s}=0.1 \mathrm{~N} / \mathrm{s}$ and a measurement interval of $0.1 \mathrm{~s}$. Digital set-up and control was performed using TestXpert II software (Zwick Roell AG, Germany), which enabled real-time measurement of the traction force. The obtained parameters were used for the calculation of average load to failure in Newton (N).

\subsubsection{Data Analysis}

The data were analysed using the Statistical Package for the Social Sciences (SPSS; version 22; IBM Inc., Somers, NY, USA) and GraphPad Prism 5.0 (San Diego, CA, USA). Continuous data are presented as mean \pm standard deviation, while incidences are presented as counts and percentages. Differences between the groups were evaluated with a two-tailed unpaired Student's t-test and with analysis of variance (ANOVA with post hoc Tukey) for continuous data. In general, Pearson's $\chi 2$-test was used for categorical values. The gene expression and biomechanics data were analysed with the Mann-Whitney $U$ test as a non-parametrical test. Potential statistical associations were evaluated with Pearson's correlation. In general, a two sided p-value $<0.05$ was considered statistically significant.

\subsection{RESULTS}

\subsubsection{Animal Mortality}

At the beginning of the experiments, all animals were in good general health, according to the 'score sheet' and 'Body condition scoring' described in the methods section. No rats died during our study due to impacts of the operative procedure or its sequelae. In the course of the experiments, 2 rats $(2.78 \%$, planned for histological analysis) had to be excluded according to our exclusion criteria; one animal had to be removed due to wound dehiscence and the other animal due to anaesthetic complications. In total, this left 70 rats (97.22\%) for inclusion in the final analyses.

\subsubsection{Gene Expression Analysis (Collagen 1A2- and 2A1- and Osteocalcin- mRNA)}

Osteocalcin expression was significantly impaired at 1 week in the NK1-R group compared to the Control group $(2.6 \pm 0.1$ fold vs. $13.5 \pm 0.4$ fold, $p=0.0002$ for $\alpha=$ $0.05)$. Collagen $1 \mathrm{~A} 2$ also showed a significant depression of expression at 1 week $(59.5 \pm 2.5$ fold vs. $228.2 \pm 33.45$ fold, $p=0.00148$ for $\alpha=0.05$ ), as well as at the 2 week time point ( $86.2 \pm 7.3$ fold vs. $170.3 \pm 3.2$ fold, $p=0.00028$ for $\alpha=0.05$ ). Collagen 2A1 expression showed a significant reduction only after 2 weeks $(1.9 \pm 0.4$ fold vs. $106.9 \pm 2.1$ fold, $p=0.000041$ for $\alpha=0.05$ ) (Figure 4.2.).

\subsubsection{Histological Analysis}

The haematoxylin-eosin staining 4 weeks after fracture did not reveal any significant differences in terms of vascularity or angiogenesis. The number and diameter of vessels were similar in both groups. We performed three microscopical measurements in each animal, but neither in trabecular diameter nor in callus diameter significant differences between both study groups were found (Figure 4.3.). 

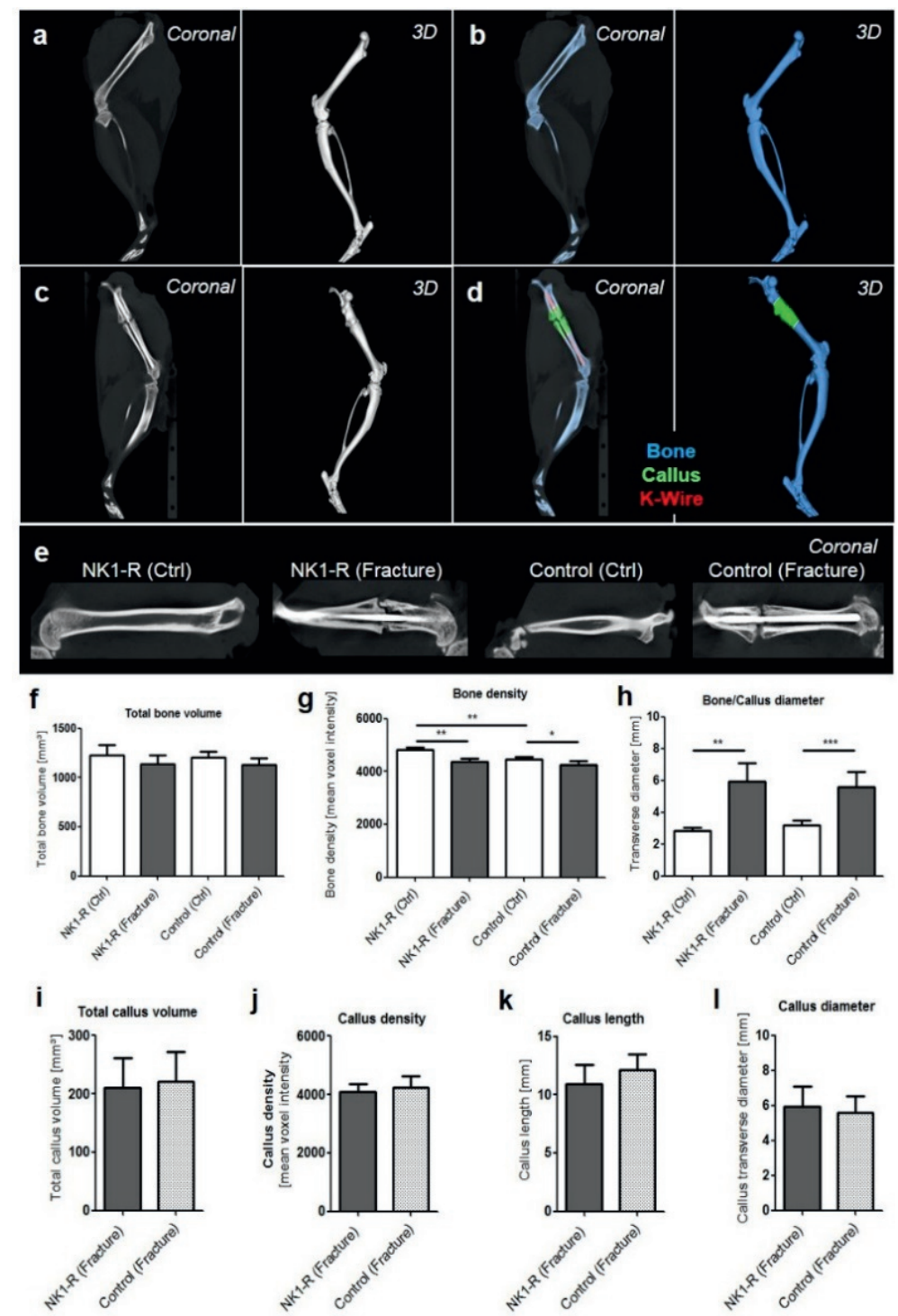

Figure 4.4. Micro-CT imaging shows the process of ossification and remodelling of the fracture site in the control group and NK1-R group. Micro-CT-imaging of the unfractured control left femur as 2D cross-sectional image in sagittal plane as well as 3D volume renderings resulting in a spatial resolution of $35 \mu \mathrm{m}$ voxel side length before (a) and after segmentation of the bone (blue) (b); micro-CT-imaging of the fractured right femur as 2D cross-sectional image in sagittal plane as well as $3 \mathrm{D}$ volume renderings resulting in a spatial resolution of $35 \mu \mathrm{m}$ voxel side length before (c) and after segmentation of the bone (blue), callus (green) and K-Wire (red) (d). 2D cross-sectional images in sagittal plane for one unfractured and fractured side of both study groups (yellow line as example for callus length and diameter) (e). MicroCT-based quantification of the total bone volume (f) and bone density (g) of the whole femur in both the fractured side and the control side of both study groups. Diameter at the fracture side compared to the diameter at the correspondence level of the unfractured side (h). Micro-CT-based quantification of the total callus volume (i), callus density (j), callus length (k) and callus diameter (l) in the fractured side of both study groups. Significance: ${ }^{*} \mathrm{p}<0.05,{ }^{* *} \mathrm{p}<0.01,{ }^{* * *} \mathrm{p}<0.005$. 


\subsubsection{Micro-CT Scanning}

The micro-computed tomography analyses conducted six weeks after fracture induction showed an almost completed process of ossification and remodelling of the fracture site in the Control group, whereas the healing process in the NK1-R group was still in progress, based on the visual analysis of the $\mu \mathrm{CT}$-scanning images, preceding the quantitative computer tomography analyses. In the quantitative computer tomography analyses, six weeks after healing, the total bone volume did not significantly differ between the fractured side with callus formation and the unfractured side without callus formation in both study groups. This implies that the comparability of the two study groups is warranted. The bone density was significantly lower for the fractured femur compared to the femur on the unfractured side in both groups (NK1-R group, $\mathrm{p}<0.01$; Control group, $\mathrm{p}<0.05$ ). Furthermore, a significant difference was noted in the transverse diameter between the fractured side (callus) and the unfractured side in both groups (NK1-R group, $\mathrm{p}<0.01$; Control group, $\mathrm{p}<0.005)$. No significant differences were observed between the Control and the NK1-R groups. The callus formation analysis did not reveal any significant differences between the two groups in terms of the volume, diameter, length, or density of the callus. (Figure 4.4.)

\subsubsection{Biomechanical Tests}

Both study groups demonstrated a decreased load to failure of the fractured femora compared to the unfractured side at 6 weeks and 3 months after fracture. By contrast, biomechanical analyses at 6 weeks (Control group $251.84 \pm 8.87 \mathrm{~N}$ vs. NK1R group $254.48 \pm 9.95 \mathrm{~N}$ ) and 3 months (Control group 247.00 $\pm 28.66 \mathrm{~N}$ and NK1-R group $224.67 \pm 19.10 \mathrm{~N}$ ) after fracture showed no significant differences in the load to failure for the unfractured side in both groups. The fractured side showed a significant $(p=0.029)$ difference in the load to failure between the NK1-R group $(69.78 \pm 8.51 \mathrm{~N})$ and the Control group $(155.45 \pm 9.32 \mathrm{~N})$ after 6 weeks. This difference was even greater at 3 months after fracture (NK1-R group: $72.50 \pm 12.09$ $\mathrm{N}$ vs. Control group: $176.33 \pm 13.44 \mathrm{~N}, \mathrm{p}=0.010$ ) (Figure 4.5.).

\subsubsection{Summary of study results}

Our main results can be summarized as follows:

(1) Blocking of the NK1-receptor for Substance P was associated with a reduced expression of different osteogenic proteins (osteocalcin, collagen 1A2, and collagen 2A1) in the early phase (the first two weeks) of fracture healing.

(2) NK1-receptor blocking impaired the normal improvement in biomechanical strength of the bone in the late phase ( 6 weeks and 3 months) after fracture.

(3) Since the quantitative callus extensions showed no significant differences, but the biomechanical strength of the healing bone was impaired, NK1-receptor blocking decreased the quality of the callus formed.

\subsection{DISCUSSION}

Fracture healing is well recognized as a complex process that is still not completely understood. However, the relationship between fracture healing and neurologic injury is well described and implies an influence of neurological neurotransmitters. 12,13 Among the various neurotransmitters, Substance P in particular is believed to 
play a central role in bone metabolism. In this context, Substance P has been shown to stimulate bone formation by osteoblastic cells through interaction with the NK1receptor 37, whereas blockage of the NK1-receptor enhanced widespread osteoporotic processes ${ }^{17}$. In the present study, our aim was to investigate what influence blocking the NK1-receptor for substance P might have on fracture healing. The important role of Substance P in bone metabolism was especially supported in the present study by the demonstration, for the first time, that selective blockage of Substance P activity by a NK1-receptor antagonist impairs the gene expression, biomechanical strength, and callus quality of a healing fracture.

\section{a Biomechanical testing (DPO 42)}
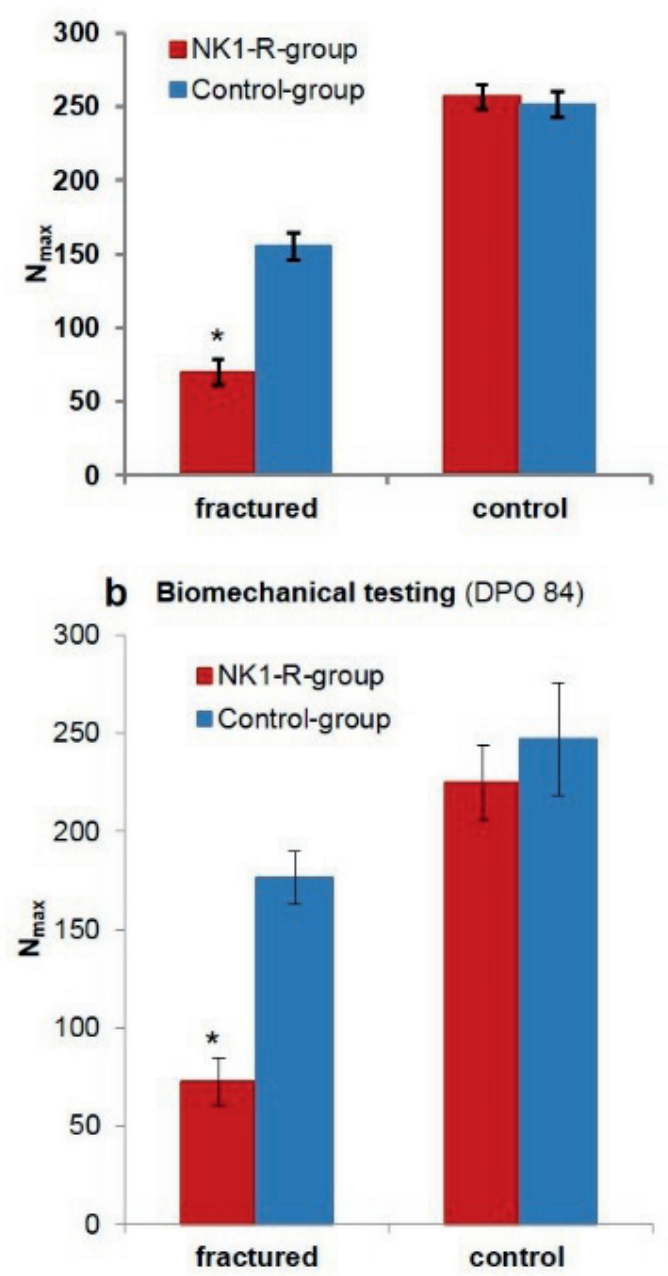

Figure 4.5. Biomechanical testing of the femora. Significant decrease in maximal load to failure $\left(\mathrm{N}_{\max }\right)$ of the femora in the NK1-R group at (a) 6 weeks $(* p=0.029)$ and (b) 3 months $(* p=0.01)$ after fracture induction. 
The decreased gene expression observed in the present study for osteocalcin, collagen 1A2, and collagen 2A1 in the early phase of callus formation after Substance $P$ receptor blockade underscores the relevance of Substance $P$ in fracture healing. These results are in line with previous RT-PCR studies that showed a similarly impaired expression of osteocalcin and collagen during fracture healing in tachykinin-deficient mice ${ }^{14}$, in sensory denervated rats ${ }^{9}$, and in rat osteoblastic cells 37.

However, beside this role in early fracture healing, Substance P seems also to have relevance for the later stages of fracture healing and remodelling of bone. In this context, we were able to show an association between NK1-receptor blockade in the first two weeks of fracture healing and a significant decrease in bone strength at 6 weeks and even at 3 months after fracture.

In previous studies, Niedermair and colleagues showed that tachykinin-1-deficient mice (Substance P knockout mice) have a reduced mechanical strength of callus after 3 weeks of fracture healing, while Apel and colleagues showed that sensory denervated rats have a reduced mechanical strength of callus after 6 weeks of fracture healing 9,14. However, none of these studies analysed the biomechanical strength at the end of the remodelling phase, as we did after 3 months. If these effects of NK1-receptor blockage are exerted in the remodelling phase or are a result based on the earlier effects of NK1-receptor blockage on fracture healing has to be explored in future studies.

Contrary to the results presented by Apel and colleagues, our findings did not indicate any significant histological differences in trabecular or callus diameter. We also did not find any significant radiological differences in quantitative callus dimensions in the $\mu \mathrm{CT}$-analyses between our two study groups, although Apel and colleagues did report significant radiological differences. Our results agree with the observations of Niedermair and colleagues, who found no radiological differences in callus dimensions between tachykinin-deficient mice and a control group ${ }^{14}$. These comparable quantitative callus dimensions between our study groups, in combination with the decrease in biomechanical strength of the bone in the NK1-R group, can only occur if the blockage of the NK1-receptor for Substance P impairs the quality of the soft and hard callus formed, although we could not substantiate this presumption with histological proof. In this context, the quality of callus is determined by the biomechanical strength of the callus and later of the remodelled bone, because the objective of fracture healing is to restore the original strength of the bone. Since Apel and colleagues performed a denervation that blocked both CGRP and Substance $P$, this might indicate that CGRP has a greater influence on the amount of callus formed, while Substance P regulates the strength and quality of callus.

The findings presented here demonstrate the indispensability of Substance P for normal and appropriate callus formation and fracture healing. Nevertheless, clarifying the exact role of Substance P in fracture healing and bone formation will require further research. Additional research is also required to assess previously postulated mechanisms of action. For instance, Niedermair and colleagues proposed crucial trophic effects of neurotransmitters on bone healing via an endogenous callus signalling loop in which chondrocytes producing Substance P and its NK1-receptor play an important role. ${ }^{14}$ They consider that the absence of Substance P results in a 
net decrease in bone formation, leading to the observed decrease in the quality of the callus.

Another interesting theory introduced by Davis and colleagues assumed that bone morphogenetic proteins (BMPs) released at the fracture site enter peripheral neurons through the damaged blood-nerve barrier. There, they induce a neuroinflammation with a release of substance $\mathrm{P}$, as well as a subsequent release of osteoprogenitor cells in cases of heterotopic ossifications. ${ }^{38}$ The results of our study could also point to a possible lead for further research into enhanced fracture healing in patients with concomitant TBI $13,39-41$, because Substance P is released early following acute injury to the CNS. This promotes a neurogenic inflammatory response that is characterized by an increase in the permeability of the blood-brain barrier ${ }^{42}$.

Our study also has some limitations. As our findings were derived from an animal model, they are not directly translatable to humans. We chose a rat model because the blockage of a neurotransmitter can only be performed in laboratory animals and because adequate blockage of the substance P-receptor with the NK1-receptor antagonist SR140333 has been previously demonstrated in this species 33 . Furthermore, the fracture model in rats is well documented in the existing literature, and the influence of NK1-receptor blockage on the complex, multifactorial process of fracture healing cannot be simulated in an in vitro experiment. Moreover, the volume measurements conducted using $\mu \mathrm{CT}$ analyses might be inaccurate due to obscuring by the intramedullary Kirschner wire, which in turn would reduce the volume measurement in the fractured bones. Therefore, the segmentation of the bone, the Kwire and the fracture callus regions was performed with a very advanced interactive method (Software Imalytics Preclinical) to minimize the error range. Even if we had removed the K-wire shortly before the $\mu \mathrm{CT}$ analysis, this removal would have left an empty space, which would not be filled with new callus tissue and therefore would probably lead to the same results. Conversely, our histology findings concerning trabecular and callus dimensions conform to our $\mu \mathrm{CT}$ findings, indicating that these results are probably reliable. Another possible limitation, was that we did not follow up the blocking capacity of SR130444 after finishing the administration after two weeks of fracture healing. We assume that the effect of SR130444 is depleted > 24 hours after application, but we did not verified it with immuno-histological tests.

In conclusion, our study findings show that blocking the NK1-receptor for substance P suppresses gene expression of important proteins in the early phases of fracture healing, decreases the quality of callus formed, and lessens the biomechanical strength of bone in the late phases of fracture healing. Therefore, based on our results, further research should be focused on the exact mechanism of action of Substance $\mathrm{P}$ in fracture healing to provide a better understanding of its potential therapeutic relevance in cases of disturbed fracture healing.

\subsection{ACKNOWLEDGEMENTS}

Source of Funding. This research project was supported by the START-Program of the Faculty of Medicine, RWTH Aachen, Germany.

Proofreading. Scribendi Inc. (Chatham, Ontario N7M 0N3 Canada) performed the proofreading of our manuscript. 


\section{Conflict of Interest Statement. The authors declare that they have no financial and / or non-financial competing interests.}

\subsection{REFERENCES}

1. Cherruau M, Facchinetti P, Baroukh B, Saffar JL. Chemical sympathectomy impairs bone resorption in rats: a role for the sympathetic system on bone metabolism. Bone 1999; 25(5): 545-51.

2. Lerner UH. Neuropeptidergic regulation of bone resorption and bone formation. Journal of musculoskeletal \& neuronal interactions 2002; 2(5): 440-7.

3. Santavirta S, Konttinen YT, Nordstrom D, et al. Immunologic studies of nonunited fractures. Acta orthopaedica Scandinavica 1992; 63(6): 579-86.

4. Hukkanen M, Konttinen YT, Santavirta S, et al. Effect of sciatic nerve section on neural ingrowth into the rat tibial fracture callus. Clinical orthopaedics and related research 1995; (311): 247-57.

5. Offley SC, Guo TZ, Wei T, et al. Capsaicin-sensitive sensory neurons contribute to the maintenance of trabecular bone integrity. Journal of bone and mineral research : the official journal of the American Society for Bone and Mineral Research 2005; 20(2): 257-67.

6. Aro H. Effect of nerve injury on fracture healing. Callus formation studied in the rat. Acta orthopaedica Scandinavica 1985; 56(3): 233-7.

7. Madsen JE, Hukkanen M, Aune AK, et al. Fracture healing and callus innervation after peripheral nerve resection in rats. Clinical orthopaedics and related research 1998; (351): 230-40.

8. Nordsletten L, Madsen JE, Almaas R, et al. The neuronal regulation of fracture healing. Effects of sciatic nerve resection in rat tibia. Acta orthopaedica Scandinavica 1994; 65(3): 299-304.

9. Apel PJ, Crane D, Northam CN, Callahan M, Smith TL, Teasdall RD. Effect of selective sensory denervation on fracture-healing: an experimental study of rats. The Journal of bone and joint surgery American volume 2009; 91(12): 2886-95.

10. Irie $\mathrm{K}$, Hara-Irie F, Ozawa H, Yajima T. Calcitonin gene-related peptide (CGRP)-containing nerve fibers in bone tissue and their involvement in bone remodeling. Microscopy research and technique 2002; 58(2): 85-90.

11. Ma WH, Liu YJ, Wang W, Zhang YZ. Neuropeptide $\mathrm{Y}$, substance $\mathrm{P}$, and human bone morphogenetic protein 2 stimulate human osteoblast osteogenic activity by enhancing gap junction intercellular communication. Brazilian journal of medical and biological research $=$ Revista brasileira de pesquisas medicas e biologicas 2015; 48(4): 299-307.

12. Morley J, Marsh S, Drakoulakis E, Pape HC, Giannoudis PV. Does traumatic brain injury result in accelerated fracture healing? Injury 2005; 36(3): 3638.

13. Hofman M, Koopmans G, Kobbe P, et al. Improved fracture healing in patients with concomitant traumatic brain injury: proven or not? Mediators of inflammation 2015; 2015: 204842.

14. Niedermair T, Kuhn V, Doranehgard F, et al. Absence of substance $P$ and the sympathetic nervous system impact on bone structure and chondrocyte differentiation in an adult model of endochondral ossification. Matrix biology : journal of the International Society for Matrix Biology 2014; 38: 2235.

15. Imai S, Matsusue Y. Neuronal regulation of bone metabolism and anabolism: calcitonin gene-related peptide-, substance P-, and tyrosine hydroxylasecontaining nerves and the bone. Microscopy research and technique 2002; 58(2): 61-9.

16. Kan L, Lounev VY, Pignolo RJ, et al. Substance P signaling mediates BMP-dependent heterotopic ossification. Journal of cellular biochemistry 2011; 112(10): 2759-72

17. Kingery WS, Offley SC, Guo TZ, Davies MF, Clark JD, Jacobs CR. A substance P receptor (NK1) antagonist enhances the widespread osteoporotic effects of sciatic nerve section. Bone 2003; 33(6): 927-36.

18. Li J, Ahmed M, Bergstrom J, Ackermann P, Stark A, Kreicbergs A. Occurrence of substance $P$ in bone repair under different load comparison of straight and angulated fracture in rat tibia. Journal of orthopaedic research : official publication of the Orthopaedic Research Society 2010; 28(12): 1643-50.

19. Liu D, Jiang LS, Dai LY. Substance P and its receptors in bone metabolism. Neuropeptides 2007; 41(5): 271-83.

20. Shih C, Bernard GW. Neurogenic substance P stimulates osteogenesis in vitro. Peptides 1997; 18(2): 323-6.

21. Wang $L$, Zhao $R$, Shi $X$, et al. Substance $P$ stimulates bone marrow stromal cell osteogenic activity, osteoclast differentiation, and resorption activity in vitro. Bone 2009; 45(2): 309-20.

22. Lerner UH, Persson E. Osteotropic effects by the neuropeptides calcitonin gene-related peptide, substance $P$ and vasoactive intestinal peptide. Journal of musculoskeletal \& neuronal interactions 2008; 8(2): 154-65.

23. Hickman DL, Swan M. Use of a body condition score technique to assess health status in a rat model of polycystic kidney disease. Journal of the American Association for Laboratory Animal Science : JAALAS 2010; 49(2): 155-9.

24. Becker JB, Arnold AP, Berkley KJ, et al. Strategies and methods for research on sex differences in brain and behavior. Endocrinology 2005; 146(4): 1650-73.

25. Milad MR, Igoe SA, Lebron-Milad K, Novales JE. Estrous cycle phase and gonadal hormones influence conditioned fear extinction. Neuroscience 2009; 164(3): 887-95.

26. Marcondes FK, Bianchi FJ, Tanno AP. Determination of the estrous cycle phases of rats: some helpful considerations. Brazilian journal of biology = Revista brasleira de biologia 2002; 62(4A): 609-14.

27. Takee A, Hirano J, Uchikura C, et al. Effects of drug treatment on bone strength and structural changes 
with aging: an experimental study in rats. Journal of orthopaedic science : official journal of the Japanese Orthopaedic Association 2002; 7(5): 544-8.

28. Bonnarens F, Einhorn TA. Production of a standard closed fracture in laboratory animal bone. Journal of orthopaedic research : official publication of the Orthopaedic Research Society 1984; 2(1): 97-101.

29. Heiner DE, Meyer MH, Frick SL, Kellam JF, Fiechtl J, Meyer RA, Jr. Gene expression during fracture healing in rats comparing intramedullary fixation to plate fixation by DNA microarray. Journal of orthopaedic trauma 2006; 20(1): 27-38.

30. Meyer RA, Jr., Desai BR, Heiner DE, Fiechtl J, Porter S, Meyer MH. Young, adult, and old rats have similar changes in mRNA expression of many skeletal genes after fracture despite delayed healing with age. Journal of orthopaedic research : official publication of the Orthopaedic Research Society 2006; 24(10): 193344.

31. Emonds-Alt X, Doutremepuich JD, Heaulme M, et al. In vitro and in vivo biological activities of SR140333, a novel potent non-peptide tachykinin NK1 receptor antagonist. European journal of pharmacology 1993; 250(3): 403-13.

32. Hegde A, Koh YH, Moochhala SM, Bhatia M. Neurokinin-1 receptor antagonist treatment in polymicrobial sepsis: molecular insights. International journal of inflammation 2010; 2010: 601098.

33. Pagan B, Isidro AA, Coppola D, et al. Effect of a neurokinin-1 receptor antagonist in a rat model of colitis-associated colon cancer. Anticancer research 2010; 30(9): 3345-53.

34. Gremse F, Stark M, Ehling J, Menzel JR, Lammers T, Kiessling F. Imalytics Preclinical: Interactive Analysis of Biomedical Volume Data. Theranostics 2016; 6(3): 328-41.

35. Oberkircher L, Schmuck M, Bergmann M, Lechler P, Ruchholtz S, Kruger A. Creating reproducible thoracolumbar burst fractures in human specimens: an in vitro experiment. Journal of neurosurgery Spine 2016; 24(4): 580-5.

36. Hollensteiner M, Samrykit M, Hess M, Fuerst D, Esterer B, Schrempf A. Inexpensive bone cement substitute for vertebral cement augmentation training. Conference proceedings : Annual International Conference of the IEEE Engineering in Medicine and Biology Society IEEE Engineering in Medicine and Biology Society Annual Conference 2016; 2016: 2202-5.

37. Goto T, Nakao K, Gunjigake KK, Kido MA, Kobayashi S, Tanaka T. Substance P stimulates latestage rat osteoblastic bone formation through neurokinin-1 receptors. Neuropeptides 2007; 41(1): 25-31.

38. Davis EL, Davis AR, Gugala Z, Olmsted-Davis EA. Is heterotopic ossification getting nervous?: The role of the peripheral nervous system in heterotopic ossification. Bone 2018; 109: 22-7.

39. Locher RJ, Lunnemann T, Garbe A, et al. Traumatic brain injury and bone healing: radiographic and biomechanical analyses of bone formation and stability in a combined murine trauma model. Journal of musculoskeletal \& neuronal interactions 2015; 15(4): 309-15.

40. Tsitsilonis S, Seemann R, Misch M, et al. The effect of traumatic brain injury on bone healing: an experimental study in a novel in vivo animal model. Injury 2015; 46(4): 661-5.

41. Cadosch D, Toffoli AM, Gautschi OP, et al. Serum after traumatic brain injury increases proliferation and supports expression of osteoblast markers in muscle cells. The Journal of bone and joint surgery American volume 2010; 92(3): 645-53.

42. Lorente L, Martin MM, Almeida T, et al. Serum substance $\mathrm{P}$ levels are associated with severity and mortality in patients with severe traumatic brain injury. Critical care 2015; 19: 192. 


\title{
Gait analysis and muscle weight analysis after lower extremity fractures in a small animal model.
}

\section{Gait \& Posture}

2020 Mar;77:207-213. doi: 10.1016/j.gaitpost.2020.01.022. [Epub 2020 Feb 6] PMID: 32058285

\author{
Martijn Hofman ${ }^{1}$ \\ Aneta Kolejewska ${ }^{1}$ \\ Johannes Greven ${ }^{1}$ \\ Hagen Andruszkow ${ }^{1}$ \\ Philipp Kobbe ${ }^{1}$ \\ Rene Tolba ${ }^{2}$ \\ Frank Hildebrand ${ }^{1 \dagger}$ \\ Martijn Poeze ${ }^{3 \dagger}$
}

1 Department of Orthopaedic Trauma and Reconstructive Surgery, University Medical Center RWTH Aachen, Germany.

2 Institute of Laboratory Animal Science, University Medical Center RWTH Aachen, Germany.

3 Department of Trauma Surgery, Maastricht University Medical Center +, the Netherlands.

$\dagger$ Authors contributed mutually 


\section{ABSTRACT}

Background: Besides adequate healing of bone and soft tissues, mobility represents a significant factor in functional outcome after lower extremity fractures. Although gait analysis is gaining clinical interest and importance in the rehabilitation of patients with fractures, it is rarely used in experimental fracture healing research. The aim of this study is to establish an accurate gait analysis method for fracture healing research in small animal models and to evaluate the influence of a lower extremity fracture on gait pattern and muscle atrophy in rats.

Research question: How does an intramedullary stabilized femur fracture influence the gait pattern and muscle atrophy during fracture healing in rats?

Methods: An isolated femur fracture with intramedullary stabilization was induced in 26 Sprague Dawley rats. Different gait parameters (e.g. intensity, print area, stand duration, duty cycle, and swing speed) were evaluated with the CatWalk gait analysis system during the fracture healing process. Furthermore, muscle weight analysis was performed at different time points.

Results: The gait analyses with the CatWalk system showed a high correlation with the osteogenesis of fracture healing in this model. Muscle atrophy increased during the early fracture healing stages and then decreased in the later stages.

Significance: We are the first to show that the CatWalk system is a useful tool to perform gait analyses after lower extremity fractures in a murine model. These results could form a basis for future gait analyses research in fracture healing studies to improve knowledge about bone regeneration and rehabilitation after lower extremity fractures. 


\subsection{INTRODUCTION}

Gait analysis is gaining clinical interest and importance in different medical disciplines. It can be of great importance especially for patients with lower extremity fractures in order to evaluate rehabilitation and mobility, thereby improving their quality of life after injury. Although gait analyses are established and integrated in large animal research protocols, they are not well established yet in small animal models. This is remarkable, as small animal models are most commonly used for fracture healing studies and are particularly suitable for them because of the general accessibility and availability of gene targeted animals and knockout models ${ }^{1-3}$. Although the CatWalk gait analysis system (Noldus Information Technology, Wageningen, Netherlands) is widely used in small animal models to investigate the impact of central and peripheral neurological injuries, neurodegenerative diseases, pain, and degenerative locomotor diseases on mobility, it has not been evaluated in a fracture healing model. However, the CatWalk system is of great interest in this field, as several clinically relevant dynamic and static gait parameters can be evaluated in a longitudinal and non-invasive way. Both gait parameters and functional outcomes are significantly influenced by muscle strength, which is often decreased after fracture and osteosynthesis because of direct damage to muscles and immobilization-triggered muscle atrophy ${ }^{4}$. Moreover, this muscle destruction is also associated with impaired bone regeneration ${ }^{5-8}$. Because of the aforementioned aspects it is important to gain experience with gait analysis methods in small animal models of fracture healing. In this study, we analysed the CatWalk system as a gait analysis method in a rat fracture model, with the aim to evaluate the influence of an intramedullary stabilized femur fracture on gait pattern and muscle atrophy over the entire fracture healing process.

\subsection{METHODS}

\subsubsection{Housing}

Our study cohort consisted of 26 adult, female Sprague Dawley rats, weighing approximately $250 \mathrm{~g}$, obtained from Envigo B.V. (Horst, Netherlands). All the animals were specifically pathogen free according to the Federation of European Laboratory Animal Science Associations recommendation. The animals were housed, and the experiments were performed at the Institute of Laboratory Animal Science, University of Aachen Medical Center, Germany, with the approval of the Governmental Animal Care and Use Committee (Landesamt für Natur, Umwelt und Verbraucherschutz, North Rhine-Westphalia, Recklinghausen, Germany; Protocol No. 84-02.04.2015.A078). The animals were housed under controlled temperature $\left(20 \pm 2^{\circ} \mathrm{C}\right)$ and air humidity $(45-65 \%)$, with a $12 \mathrm{~h}$ light-dark cycle and a light intensity of $<200 \mathrm{~lx}$. Food and water were offered ad libitum. Prior to study inclusion, all the animals were kept in groups for 1 week in the laboratory premises to allow acclimatization. Throughout the entire experiment, they underwent physical examinations according to a score sheet documentation ${ }^{9}$ and the Body Condition Scoring system by Hickman and Swan ${ }^{10}$ to obtain their general health. Because of the protective effects of female hormones in cases of inflammatory stimuli, all the animals were confirmed to be in the same 'metestrus' phase of the menstrual cycle, as this phase is characterized by low oestrogen and progesterone levels ${ }^{11}$. The 
menstrual cycle phase was identified by the assessment of vaginal swabs according to Marcondes and colleagues ${ }^{12}$.

\subsubsection{Experimental design}

In all the animals, an intramedullary pin was inserted after anaesthesia induction and a standardized femoral fracture was induced at the right side. The animals were then randomly divided into 2 study groups: one in which gait analyses were performed $(n=6)$ and one in which muscle weight analyses were performed $(n=$ 20). All the animals from the gait analysis study track underwent gait analysis at 7 time points during the fracture healing process, including a baseline measurement before the intervention. The muscle weight analyses were performed in randomly assigned subgroups of 5 animals at 4 time points during the fracture healing process (Figure 5.1.). The reasons for excluding animals were as follows: death from anaesthetic complications, open fracture, comminuted fracture, implant failure, wound dehiscence or infection, impossibility to perform 3 adequate runs in the CatWalk system.

\subsubsection{Anaesthesia and pain management}

The animals received buprenorphine hydrochloride $(0.03-0.05 \mathrm{mg} / \mathrm{kg}$ s.c.) as a multimodal analgesic $30 \mathrm{~min}$ preoperatively. The operative procedures were performed under general anaesthesia induced with ketamine $(100 \mathrm{mg} / \mathrm{kg}$ i.p.) and xylazine ( $2 \% ; 10 \mathrm{mg} / \mathrm{kg}$ i.p.) and, if necessary, extended with isoflurane inhalation (2.0-2.5 Vol.\%). The toe pinch reflex was used to assure adequate anaesthesia. Postoperative analgesia was assured with buprenorphine hydrochloride (0.03$0.05 \mathrm{mg} / \mathrm{kg}$ s.c.) every $6 \mathrm{~h}$ for the first $24-48 \mathrm{~h}$. Subsequently, it was given twice daily during the first 3 weeks. Furthermore, in the first postoperative week, the drinking water was supplemented with metamizole $(1 \mathrm{ml} / 300 \mathrm{ml})$.

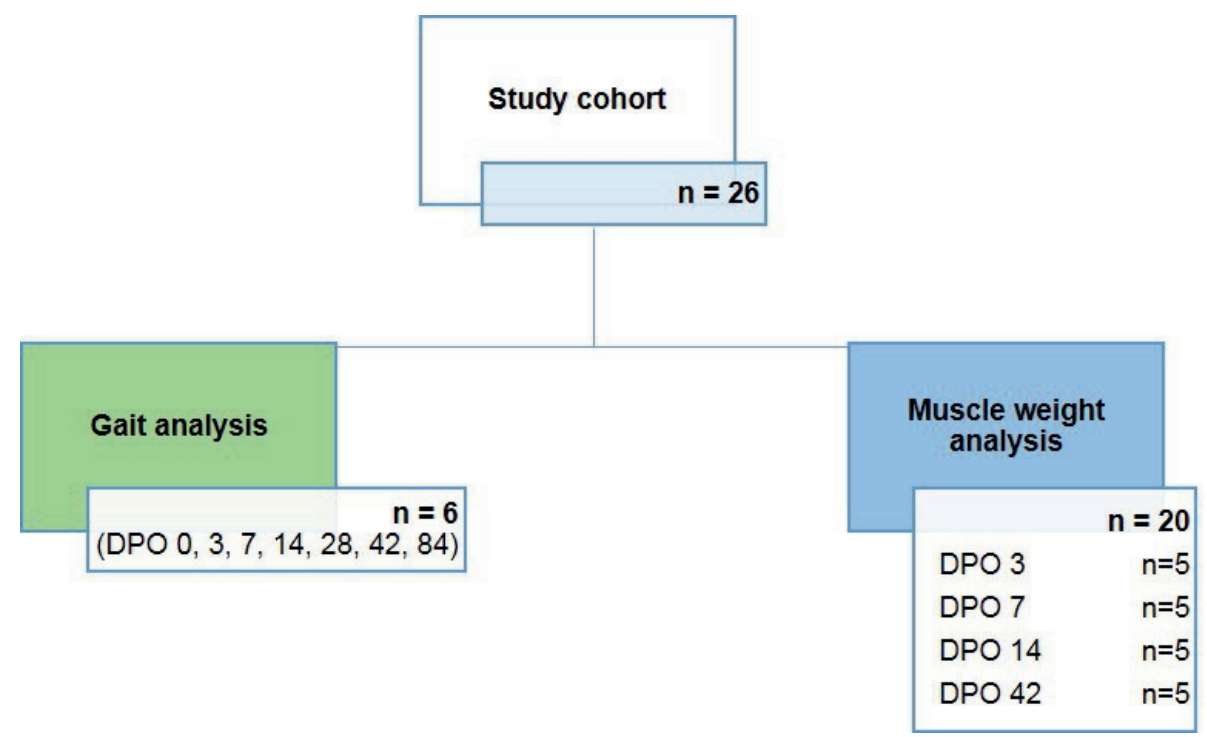

Figure 5.1. Subgroup division and time points of analyses. Subdivision of study cohort in 2 study groups (gait analysis and muscle weight analysis) and subsequent division according to performed analysis at different time points. (DPO: days postoperative) 
The animals were evaluated postoperatively 3 times per day according to the score sheet evaluation ${ }^{9}$.

\subsubsection{Standardized femoral fracture}

After anaesthesia induction, the animals were placed on a heated pad $\left(37^{\circ} \mathrm{C}\right)$ and their eyes were covered with a moistening ointment. Their right hind leg was shaved, disinfected, and draped. Then, a parapatellar incision was made, the patella was everted laterally, and a $1 \mathrm{~mm}$ stainless-steel intramedullary Kirschner wire (Königsee Implantate $\mathrm{GmbH}$, Allendorf, Germany) was inserted in a retrograde manner. Its placement was confirmed by fluoroscopy. The Kirschner wire was cut flush with the intercondylar notch, and the proximal end was bent over the greater trochanter, cut, and hidden subcutaneously. The patella was repositioned and the wounds were sutured (Ethicon Inc., Somerville, NJ, USA) in layers. Fracture induction was performed with a blunt guillotine according to the method of Bonnarens and Einhorn ${ }^{13}$. A fluoroscopic evaluation of the fractured side was performed directly after fracture induction. Fracture healing, defined as bridging of 3 out of 4 cortices, was assessed radiologically at 42 days postoperative (DPO).

\subsubsection{Gait analysis}

\subsubsection{Description of technique and measured parameters}

Gait analysis was carried out using the CatWalk system (version XT 8.1) - an automated gait analysis system which can measure dynamic (i.e. stand duration, duty cycle, and swing speed) and static (i.e. print area and intensity) gait parameters of small animals. The system consists of an enclosed $1.3-\mathrm{m}$-long walkway of an enlightened glass plate. The animal crosses this walkway from left to right, and each individual footprint on the enlightened glass plate is detected by a camera placed below and registered by the CatWalk software ${ }^{14,15}$ (Figure 5.2.). From the registered gait data, the following parameters were calculated for both hindlimbs and their ratios between the hindlimbs: intensity (average pressure of print for the given paw (arbitrary units)); print area (total print area for the given paw $\left(\mathrm{mm}^{2}\right)$ ); stand duration (time spent bearing weight per step for the given paw (s)); duty cycle (percentage of the time spent bearing weight in each walk cycle for the given paw (\%)); and swing speed (phase(s) of the limb between steps for the given paw). These data were collected from the CatWalk software after performing the 'auto classify' function. Additionally, these data were edited according to the systematic manual classification method described by Chen and colleagues ${ }^{15}$.

\subsubsection{Pre-traumatic treatment and time point of measurements}

Preoperatively, the animals were trained twice daily on the CatWalk system for 2 weeks to get used to the test situation. Their cages were put at the end of the corridor with reward food pellets, and they were set at the other end of the corridor. They were able to walk freely to their own cages. The goal of this training was that the animals could perform 5 successful runs. A successful run is a passage with no explicit stops, with a velocity variation of no more than $60 \%$ and a maximum velocity of no more than $400 \mathrm{~mm} / \mathrm{s}$. For the final analysis, the animals needed to perform 3 successful runs at each time point. To guarantee a standardized environment, they were trained by the same person and the room set-up was the same during all the 
runs. Post-traumatic gait analysis was performed throughout the entire fracture healing process at DPO 3, 7, 14, 28, 42, and 84. A baseline measurement was performed preoperatively at DPO 0.

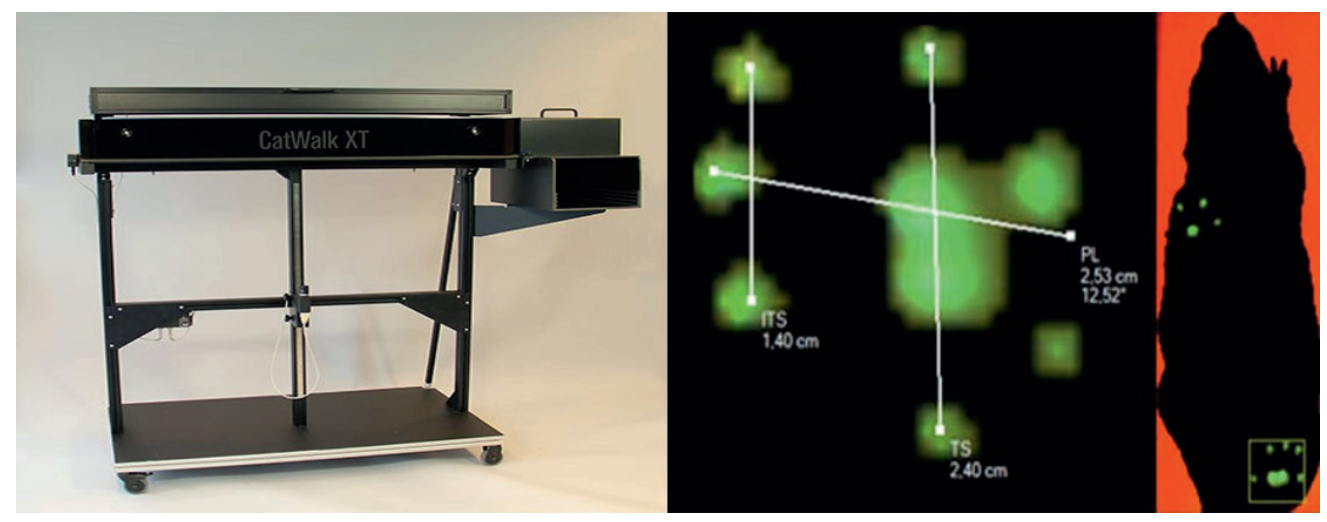

Figure 5.2. CatWalk gait analysis system. CatWalk walkway device (left) and footprint identification from the real-life imaging of the CatWalk system. (From Noldus Information Technology, www.noldus.com.)

\subsubsection{Muscle weight analysis}

Muscle weight analyses were performed at 4 time points (DPO 3, 7, 14, and 42) throughout the fracture healing process. At each time point, 5 rats were euthanized with an overdose of isoflurane and subsequent cervical dislocation, the lateral vastus muscle was harvested in toto on both sides (the fractured right leg and unfractured left leg), and then the 'dry' muscle weight was measured after 3 days of dehumidifying in an incubator at $50^{\circ} \mathrm{C}$. The ratio of the muscle mass at the fractured side compared to the unfractured side in each animal was evaluated.

\subsubsection{Endpoint and power analysis}

The main endpoint of our study was a normal gait pattern according to the different parameters of the CatWalk system. As these gait analyses with the CatWalk system were never performed before in rats with femur fractures, we based our power analysis on the study by Apel and colleagues ${ }^{16}$. In this study, the rats in which a femoral fracture was induced after intramedullary stabilization were also evaluated for fracture healing parameters. Their power analysis was based on an average maximal load to failure of rat femora of $131.3 \pm 4.9 \mathrm{~N}$, which was described as the load to failure of normal rat femora by Takee and colleagues 16,17 . According to this calculation, the animal groups were required to include a minimum of 4 rats to achieve a $95 \%$ power for detecting a difference of $10 \mathrm{~N}$, with $\alpha=0.05$. In order to compensate for the potential loss of animals, a sample size of 6 animals per group was chosen. The power analysis was performed with G*Power (version 3.1; University of Düsseldorf, Germany). The secondary outcome parameter was the extent of muscle weight loss during the fracture healing process. 


\subsubsection{Data analysis}

The data were analysed using SPSS (version 25; IBM Inc., Somers, NY, USA). The continuous values were presented as median with interquartile ranges (25-IQR and 75-IQR). The muscle weight was described by mean and standard deviation. Differences between the groups were evaluated with Mann-Whitney's U-test for continuous data. The power analysis was performed with $G^{*}$ Power. In general, a 2sided p-value $<0.05$ was considered to be significant.

Table 5.1. Gait parameters. Percentage change to baseline values of gait parameter (intensity, print area, stand duration, duty cycle, and swing speed) median (MED) and interquartile range (IQR). Relative changes to baseline values for the fractured hind leg (above) and for the ratio of the fractured to unfractured side (R/L; bottom) in percentages.

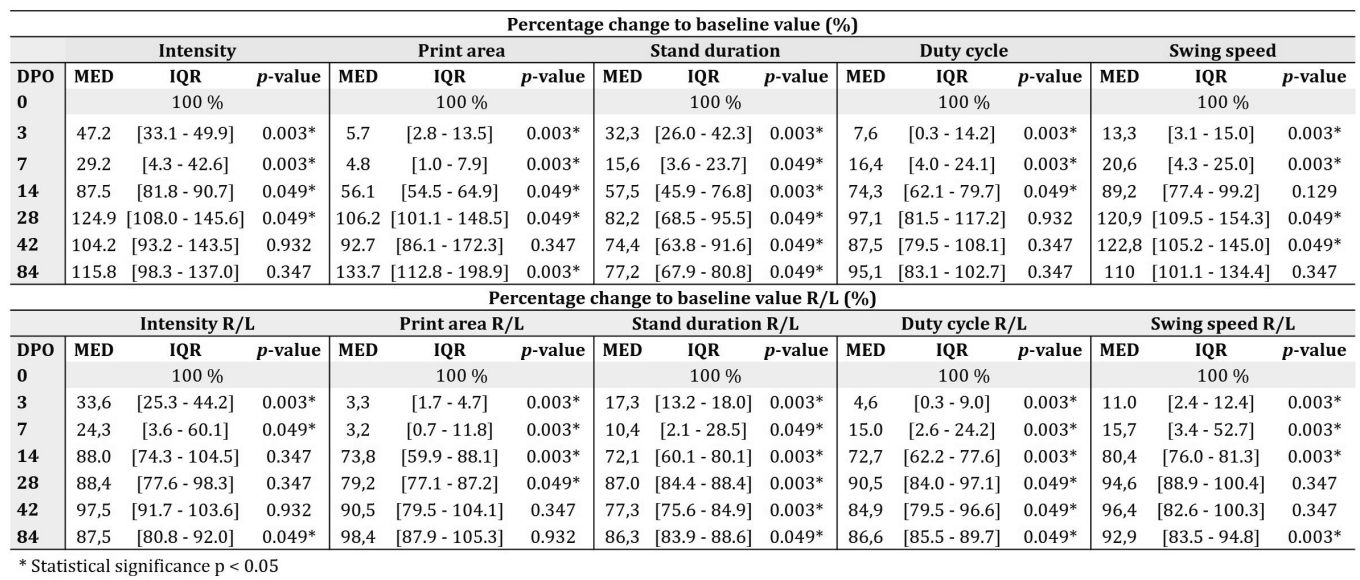

\subsection{RESULTS}

The radiological evaluation of fracture healing at DPO 42 showed bridging of 3 out of 4 cortices and no significant dislocation in all subjects. No animals were needed to be excluded according to our exclusion criteria.

\subsubsection{Gait analysis}

The results were represented as relative changes to baseline values in percentages. We evaluated 5 gait parameters (intensity, print area, stand duration, duty cycle, and swing speed) for the fractured hind leg and also for the ratio of the fractured to unfractured hind leg (marked with 'R/L'). In the early fracture healing stages (DPO $0-14$ ), the fractured hind leg demonstrated significant changes to baseline values for all gait parameters. In the later fracture healing stages (DPO 14-84), these values normalized to baseline values (stand duration and duty cycle) or even exceeded these values (intensity, print area, and swing speed) (Table 5.1.; Figure 5.3.). 


\subsubsection{Muscle weight analysis}

The muscle weight of the unfractured side increased constantly from DPO 3 to 42, whereas the muscle weight of the fractured side showed a depression in the first week after fracture induction, with a subsequent increase over the next 5 weeks. Comparing the fractured with unfractured side we could demonstrate a significant decreased muscle weight of the fractured side from DPO 7 throughout the entire fracture healing process (Table 5.2.; Figure 5.4.).

\subsection{DISCUSSION}

From clinical experience it is well known that the period of immobilization after stabilization of lower extremity fractures depends on different patient- and fracturespecific factors. Generally, postoperative weight bearing is increased as soon as possible to prevent complications, stimulate fracture healing, and regain mobility, muscle strength, and function. However, objective gait analysis methods are not widely integrated in the rehabilitation of patients with lower extremity fractures. Because data on post-traumatic or -operative gait patterns are important to improve the recovery of patients after lower extremity fractures, it is gratifying to see that gait analysis after lower extremity fractures is gaining increased interest and importance. Therefore, it is even more remarkable that the gait analysis methods in the most commonly used small animal models of fracture healing are almost absent. About $50 \%$ of all animal fracture healing studies are performed in small animal models ${ }^{2}$, but only 1 study describes a gait analysis method for lower extremity fractures in rats ${ }^{3}$. However, this study by Histing and colleagues performs gait analysis with a treadmill at only 1 time point in the fracture healing process. In the present study, we therefore introduced for the first time - to the best of our knowledge - the CatWalk system for gait analyses in a rat model of femoral fracture healing. Additionally, the post-traumatic course of muscle weight changes was evaluated.

Our main results can be summarized as follows:

1. Intramedullary stabilized femur fractures influence the gait pattern in the early fracture healing stages.

2. Intramedullary stabilized femur fractures are associated with muscle atrophy in the early fracture healing stages.

3. The changes in gait parameters according to the CatWalk system are associated with the progress of fracture healing.

Complete bone bridging of a fracture in rats takes approximately 5 weeks, and torsional stiffness increases to $30-100 \%$ in this period ${ }^{2,18,19}$. In our study, we found a close association between the normal fracture healing process and the normalization of both gait parameters and muscle weight. In the first 2 weeks after fracture induction, all gait parameters (i.e. intensity, print area, stand duration, duty cycle, and swing speed) were reduced compared to the baseline measurements, but from the 3rd to 6th week of fracture healing, the gait parameters returned to baseline values. Archdeacon and colleagues showed similar results in a clinical study of patients with femoral fractures ${ }^{4}$. This and other study groups, therefore, emphasized the importance of gait dysfunction in short- and long-term functional outcomes 4,20 24. The values of intensity, print area, and swing speed even exceed the basic values 
at DP0 28 - 84 and this could be due to new formed callus which has a bigger diameter and is possibly stronger than the original femur before fracture induction.

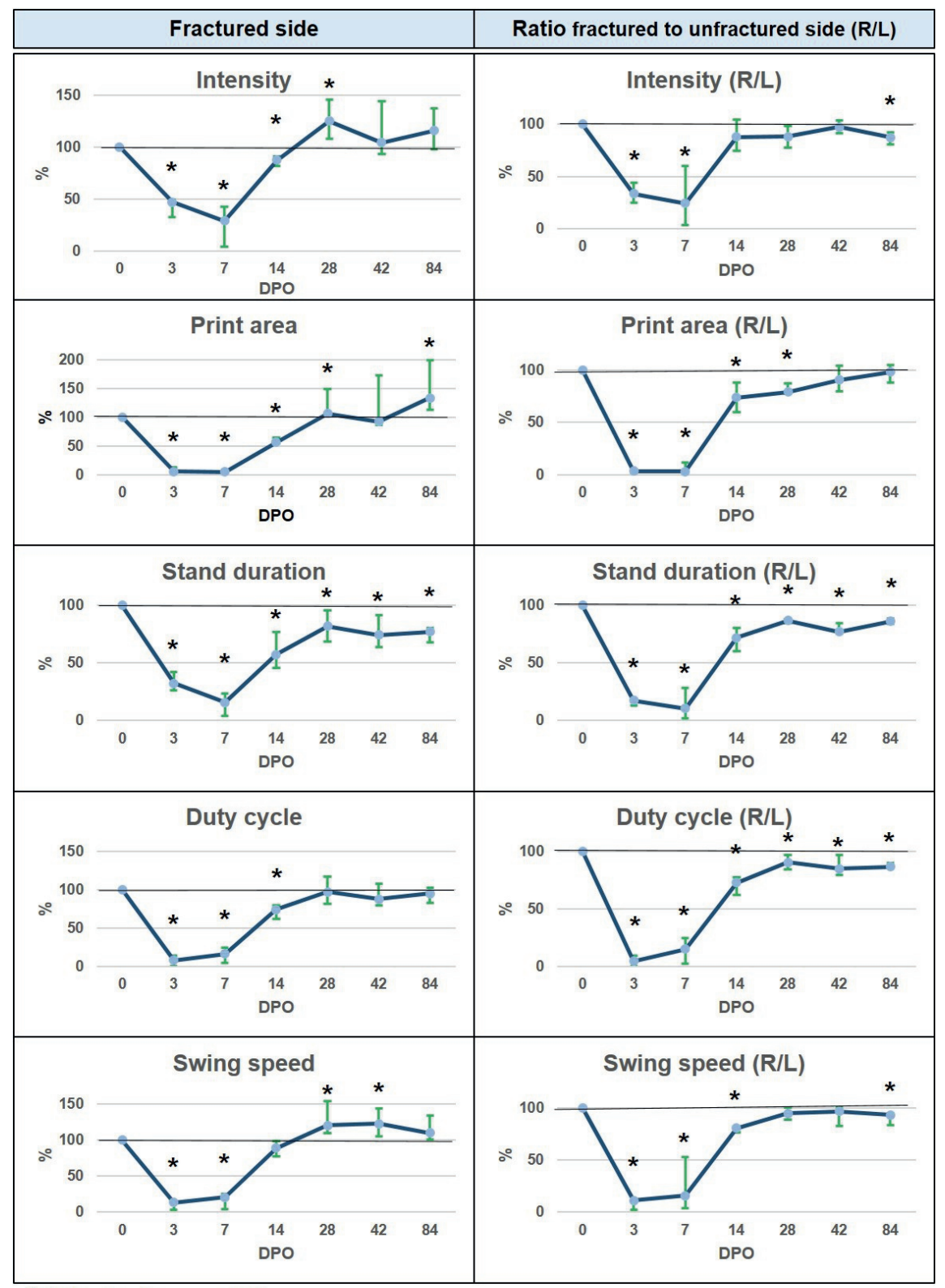

* Statistical significance $p<0.05$

Figure 5.3. Gait analysis in graphs. Percentage change to baseline values of gait parameters. Visualization of relative changes to baseline values of all 5 gait parameters (intensity, print area, stand duration, duty cycle, and swing speed) for the fractured hind leg (left column) and for the ratio of the fractured to unfractured side (R/L; right column). 
Table 5.2. Comparison of the muscle weight of the fractured and unfractured side within the same animal. Significant differences between vastus lateralis muscle weight of the fractured and unfractured side of the same animal.

\begin{tabular}{lcccc}
\hline \multicolumn{5}{c}{ Mucle weight of lateral vastus muscle in $\mathbf{~}$} \\
Mean $( \pm \mathrm{SD})$ & $\boldsymbol{\Delta}$ & $\boldsymbol{p}$-Value \\
\hline $\mathbf{D P O}$ & Unfractured side & Fractured side & 0.038 & 0.310 \\
$\mathbf{3}$ & $0.426( \pm 0.045)$ & $0.388( \pm 0.025)$ & 0.119 & $0.029^{*}$ \\
$\mathbf{7}$ & $0.454( \pm 0.018)$ & $0.335( \pm 0.058)$ & 0.086 & $0.032^{*}$ \\
$\mathbf{1 4}$ & $0.482( \pm 0.050)$ & $0.396( \pm 0.027)$ & 0.081 & $0.032^{*}$ \\
\hline $\mathbf{4 2}$ & $0.486( \pm 0.031)$ & $0.405( \pm 0.053)$ & & \\
\hline
\end{tabular}

* Statistical significance $\mathrm{p}<0.05$

With our study we are the first to demonstrate that the gait patterns of rats resemble those of patients with lower extremity fractures; only the healing process - and thus the impaired mobility - lasts longer in human beings. Because studies on small animal models are the most commonly used experimental models in fracture healing research, this observation can form an important basis for future fracture healing research. In the early fracture healing stages, the muscle weight of the fractured side was reduced in coherence with the impaired gait pattern.

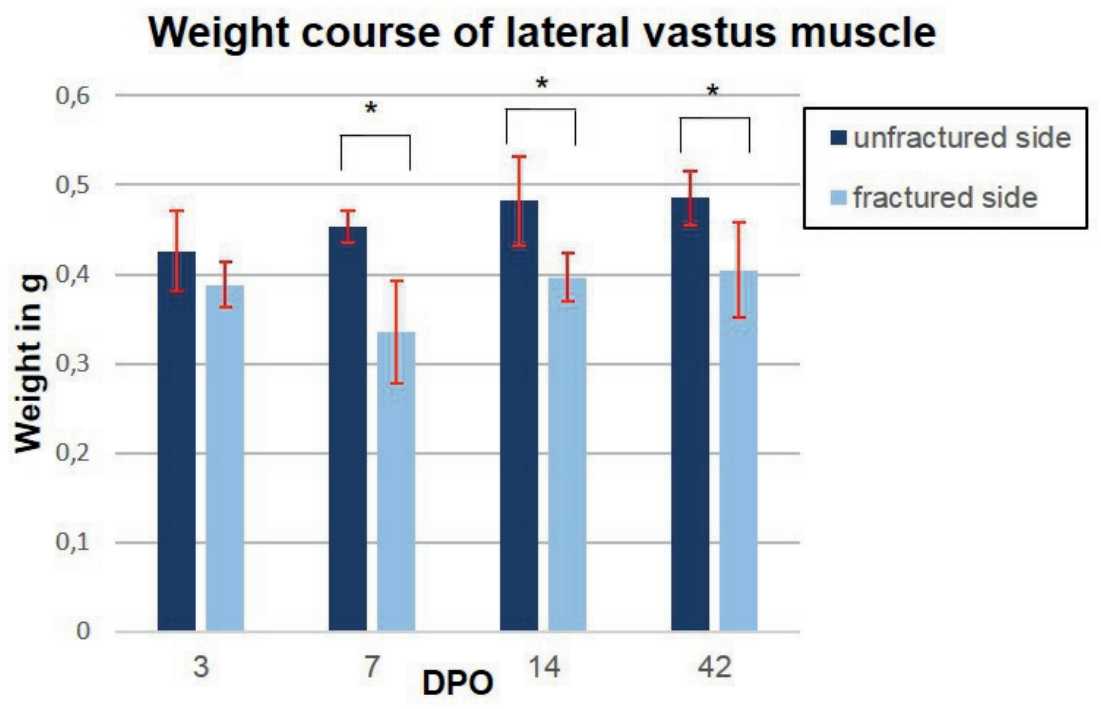

Figure 5.4. Muscle weight of the lateral vastus muscle. Muscle weight significantly decreases at the fractured side in the early fracture healing stages. 
This was most likely caused by immobilization and, particularly at the very beginning, also by the impact of fracture induction and intramedullary stabilization. This is in line with clinical studies which showed significant muscle atrophy even after short periods of immobilization, with a negative influence on fracture biomechanics $5,25-27$. In the later fracture healing stages, as the gait pattern of the animals normalized and the mobility improved, muscle atrophy decreased and muscle weight shows a tendency to return to normal values. This pattern of muscle atrophy is also demonstrated in literature ${ }^{5}$. It is stated that the influence of this muscle atrophy goes beyond the direct effect on fracture healing, because it can impair gait patterns and general remobilization, thereby prolonging the rehabilitation process and even decreasing the functional outcome and quality of life of these patients $4,28-30$. Overall, the CatWalk system adequately analyses and clinically reflects the gait pattern development in small animal models of fracture healing. It offers advantages over treadmills: First, the CatWalk system uses a walkway set-up, which matches the normal gait pattern of these animals much better. Second, both static and dynamic gait parameters can be evaluated at the same time. As Kappos and colleagues described earlier for functional nerve recovery in a small animal model, the CatWalk system is the only technique which can reliably analyse dynamic gait parameters, including swing speed, stand duration, and interlimb coordination ${ }^{14}$. The improvement of gait parameters is especially important in rehabilitation after lower limb fractures, because it has been proved that an isolated femur fracture, and the subsequent impaired gait pattern, also influences the kinematics and kinetics of the adjacent joints, which is time dependent and can influence the long-term functional outcome ${ }^{4}$. This underlines the importance of the described CatWalk system. Third, when the animals are trained well, the registration is accurate and this system can provide clinically relevant and extensive data on gait patterns. The current study also has some limitations. Although we demonstrated a good correlation between our results and clinical findings, small animal models are not directly transferable to the human situation. However, more than $50 \%$ of experimental fracture healing studies are performed in small animal models. Therefore, our results are important as they confirm that gait analysis is a translationally relevant technique to gain information on the fracture healing process and posttraumatic mobilization. Moreover, we did not perform baseline measurements of the muscle weight in healthy animals of the same weight as a reference measurement. This was with intent, because muscle weight is extremely individual specific, and therefore, we were more interested in the differences in the muscle weight of the fractured against unfractured side in 1 animal.

\subsection{CONCLUSIONS}

We demonstrated for the first time that the CatWalk system is practicable and useful to assess both static and dynamic gait parameters in a non-invasive, longitudinal manner in an experimental small animal model of fracture healing. Our pre-clinical findings are in line with clinical observations in patients with lower extremity fractures. Therefore, this system has the potential to become a standard gait analysis method in fracture healing research in experimental small animal models, thereby improving knowledge about behavioural and locomotor recovery after lower extremity fractures. 


\subsection{ACKNOWLEDGEMENTS}

Funding. This work was supported by the START-Program of the Faculty of Medicine, RWTH Aachen University, Germany (Grant nr. 691451). The sponsor had no role in the design of this study; collection, analysis, and interpretation of data; writing of the manuscript; or decision to submit the article for publication.

Proofreading. Scribendi Inc. (Chatham, Ontario N7M 0N3 Canada) performed the proofreading of our manuscript.

CRediT authorship contribution statement. Martijn Hofman: Conceptualization, Methodology, Validation, Investigation, Resources, Data curation, Writing - original draft, Writing - review \& editing, Visualization, Project administration, Funding acquisition. Aneta Kolejewska: Formal analysis, Investigation, Data curation. Johannes Greven: Methodology, Validation, Formal analysis, Investigation, Data curation, Project administration. Hagen Andruszkow: Formal analysis, Writing review \& editing. Philipp Kobbe: Conceptualization, Methodology. Rene Tolba: Resources, Writing - review \& editing. Frank Hildebrand: Conceptualization, Writing - review \& editing, Supervision. Martijn Poeze: Conceptualization, Writing - review \& editing, Supervision.

Declaration of Competing Interest. The authors declare that they have no competing interests.

\subsection{REFERENCES}

1. Jacenko O, Olsen BR. Transgenic mouse models in studies of skeletal disorders. The Journal of rheumatology Supplement 1995; 43: 39-41.

2. Garcia P, Histing T, Holstein JH, et al. Rodent animal models of delayed bone healing and non-union formation: a comprehensive review. European cells \& materials 2013; 26: 1-12; discussion -4.

3. Histing $\mathrm{T}$, Kristen $\mathrm{A}$, Roth $\mathrm{C}$, et al. In vivo gait analysis in a mouse femur fracture model. Journal of biomechanics 2010; 43(16): 3240-3.

4. Archdeacon M, Ford KR, Wyrick J, et al. A prospective functional outcome and motion analysis evaluation of the hip abductors after femur fracture and antegrade nailing. Journal of orthopaedic trauma 2008; 22(1): 3-9.

5. Hao Y, Ma Y, Wang X, Jin F, Ge S. Short-term muscle atrophy caused by botulinum toxin-A local injection impairs fracture healing in the rat femur. Journal of orthopaedic research : official publication of the Orthopaedic Research Society 2012; 30(4): 574-80.

6. Li G, Wang L, Jiang Y, et al. Upregulation of Akt signaling enhances femoral fracture healing by accelerating atrophic quadriceps recovery. Biochimica et biophysica acta Molecular basis of disease 2017; 1863(11): 2848-61.

7. Utvag SE, Iversen KB, Grundnes O, Reikeras 0. Poor muscle coverage delays fracture healing in rats. Acta orthopaedica Scandinavica 2002; 73(4): 471-4.

8. Utvag SE, Grundnes O, Rindal DB, Reikeras 0. Influence of extensive muscle injury on fracture healing in rat tibia. Journal of orthopaedic trauma 2003; 17(6): 430-5.
9. Morton DB, Griffiths PH. Guidelines on the recognition of pain, distress and discomfort in experimental animals and an hypothesis for assessment. The Veterinary record 1985; 116(16): 431-6.

10. Hickman DL, Swan M. Use of a body condition score technique to assess health status in a rat model of polycystic kidney disease. Journal of the American Association for Laboratory Animal Science : JAALAS 2010; 49(2): 155-9.

11. Becker JB, Arnold AP, Berkley KJ, et al. Strategies and methods for research on sex differences in brain and behavior. Endocrinology 2005; 146(4): 1650-73.

12. Marcondes FK, Bianchi FJ, Tanno AP. Determination of the estrous cycle phases of rats: some helpful considerations. Brazilian journal of biology = Revista brasleira de biologia 2002; 62(4A): 609-14.

13. Bonnarens F, Einhorn TA. Production of a standard closed fracture in laboratory animal bone. Journal of orthopaedic research : official publication of the Orthopaedic Research Society 1984; 2(1): 97-101.

14. Kappos EA, Sieber PK, Engels PE, et al. Validity and reliability of the CatWalk system as a static and dynamic gait analysis tool for the assessment of functional nerve recovery in small animal models. Brain and behavior 2017; 7(7): e00723.

15. Chen H, Du J, Zhang Y, Barnes K, Jia X. Establishing a reliable gait evaluation method for rodent studies. Journal of neuroscience methods 2017; 283: 92-100.

16. Apel PJ, Crane D, Northam CN, Callahan M, Smith TL, Teasdall RD. Effect of selective sensory denervation on fracture-healing: an experimental 
study of rats. The Journal of bone and joint surgery American volume 2009; 91(12): 2886-95.

17. Takee A, Hirano J, Uchikura C, et al. Effects of drug treatment on bone strength and structural changes with aging: an experimental study in rats. Journal of orthopaedic science : official journal of the Japanese Orthopaedic Association 2002; 7(5): 544-8.

18. Kratzel C, Bergmann C, Duda G, Greiner S, Schmidmaier G, Wildemann B. Characterization of a rat osteotomy model with impaired healing. $B M C$ musculoskeletal disorders 2008; 9: 135.

19. Wray JB, Lynch CJ. The vascular response to fracture of the tibia in the rat. The Journal of bone and joint surgery American volume 1959; 41-A: 1143-8.

20. Pfeufer D, Zeller A, Mehaffey S, Bocker W, Kammerlander C, Neuerburg C. Weight-bearing restrictions reduce postoperative mobility in elderly hip fracture patients. Archives of orthopaedic and trauma surgery 2019; 139(9): 1253-9.

21. Siu AL, Penrod JD, Boockvar KS, Koval K, Strauss E, Morrison RS. Early ambulation after hip fracture: effects on function and mortality. Archives of internal medicine 2006; 166(7): 766-71.

22. Kamel HK, Iqbal MA, Mogallapu R, Maas D, Hoffmann RG. Time to ambulation after hip fracture surgery: relation to hospitalization outcomes. The journals of gerontology Series A, Biological sciences and medical sciences 2003; 58(11): 1042-5.

23. Kammerlander C, Gosch M, KammerlanderKnauer U, Luger TJ, Blauth M, Roth T. Long-term functional outcome in geriatric hip fracture patients. Archives of orthopaedic and trauma surgery 2011; 131(10): 1435-44.
24. Frenkel Rutenberg $\mathrm{T}$, Vitenberg $\mathrm{M}$, Haviv $\mathrm{B}$, Velkes S. Timing of physiotherapy following fragility hip fracture: delays cost lives. Archives of orthopaedic and trauma surgery 2018; 138(11): 1519-24.

25. Dirks ML, Wall BT, van de Valk B, et al. One Week of Bed Rest Leads to Substantial Muscle Atrophy and Induces Whole-Body Insulin Resistance in the Absence of Skeletal Muscle Lipid Accumulation. Diabetes 2016; 65(10): 2862-75.

26. Wall BT, Dirks ML, van Loon LJ. Skeletal muscle atrophy during short-term disuse: implications for age-related sarcopenia. Ageing research reviews 2013; 12(4): 898-906.

27. Fox KM, Magaziner J, Hawkes WG, et al. Loss of bone density and lean body mass after hip fracture. Osteoporosis international : a journal established as result of cooperation between the European Foundation for Osteoporosis and the National Osteoporosis Foundation of the USA 2000; 11(1): 31-5.

28. Lamb SE, Oldham JA, Morse RE, Evans JG. Neuromuscular stimulation of the quadriceps muscle after hip fracture: a randomized controlled trial. Archives of physical medicine and rehabilitation 2002; 83(8): 1087-92.

29. Yang SD, Ning SH, Zhang LH, Zhang YZ, Ding WY, Yang DL. The effect of lower limb rehabilitation gymnastics on postoperative rehabilitation in elderly patients with femoral shaft fracture: A retrospective case-control study. Medicine 2016; 95(33): e4548.

30. Sylliaas H, Brovold T, Wyller TB, Bergland A Progressive strength training in older patients after hip fracture: a randomised controlled trial. Age and ageing 2011; 40(2): 221-7. 


\section{Arginine availability in reamed intramedullary aspirate as predictor of outcome in non-union healing, a pilot study. \\ Manuscript submitted}

Dennis M. Meesters ${ }^{1,2}$

Karolina A.P. Wijnands ${ }^{1,2}$

Hans M.H. van Eijk ${ }^{1,2}$

Clint Boymans ${ }^{1}$

Martijn Hofman ${ }^{3}$

Frank Hildebrand ${ }^{3}$

Jan P.A.M. Verbruggen ${ }^{1}$

Peter R.G. Brink ${ }^{1}$

Martijn Poeze ${ }^{1,2}$

${ }^{1}$ Department of General Surgery and Trauma Surgery, Maastricht University Medical Center +, the Netherlands.

2 NUTRIM School for Nutrition and Translational Research in Metabolism, Maastricht, the Netherlands

${ }^{3}$ Department of Orthopaedic Trauma and Reconstructive Surgery, University Medical Center RWTH Aachen, Germany 


\section{ABSTRACT}

Aims: Normal fracture healing and non-union development are influenced by a wide range of biological factors. Adequate concentrations of amino acids, especially arginine, are known to be important during normal bone healing. We hypothesize that the bone arginine availability in autologous bone marrow grafting by use of the reamer-irrigatoraspirator (RIA) procedure is a marker of bone healing capacity in patients treated for non-union.

Patients and methods: 17 patients treated for atrophic long bone non-union by autologous bone grafting by RIA procedure were included and divided into two groups: successful treatment of non-union and unsuccessful, and were compared to 8 control patients after normal fracture healing. Reamed bone marrow aspirate from an unaffected bone distant to the non-union was obtained and amino acids were measured using high-performance liquid chromatography. RNA was isolated for quantitative polymerase chain reaction analysis of relevant enzymes in the arginine metabolism.

Results: Arginine and ornithine concentrations were higher in patients with successful bone healing after RIA in comparison with an unsuccessful healing. Ornithine concentrations were lower in all non-union patients compared to control patients, while citrulline concentrations were increased. Nos2 expression was significantly increased in all RIA treated patients, and higher in patients with a successful outcome when compared with unsuccessful outcome after RIA. Nos3 expression was not detectable in RIA treated patients. Arg1 was lower in all RIA treated patients when compared to control samples.

Conclusions: The results indicate an influence of the arginine-nitric oxide metabolism in bone marrow, harvested using reamed intramedullary aspirate on the outcome of nonunion treatment, with indications for a prolonged inflammatory response in patients with unsuccessful bone grafting therapy. Determination of arginine concentrations and Nos2 expression could be used as predictor for successful treatment of autologous bone grafting in non-union treatment. 


\subsection{INTRODUCTION}

Non-union development occurs in $10-15 \%{ }^{1}$ of patients with long bone fractures. This incidence can increase up to $45 \%$ depending on risk factors such as fracture configuration and infection ${ }^{2,3}$. Next to a major decrease in quality of life, non-union is accompanied by high socio-economic costs caused by multiple surgical interventions needed for adequate treatment ${ }^{4}$. A regular treatment option for long bone non-unions is the use of autologous bone grafting by reamed-irrigation-aspiration (RIA) for harvesting of bone and bone marrow. This technique fulfils the requirements for adequate bone grafting stated in the Diamond Concept 5,6 . The general risk of persisting failure of bone healing after bone grafting procedure for non-union using the RIA procedure is around $10-18 \%{ }^{7-9}$. Although the molecular pathogenesis of non-union remains unclear, a better understanding may provide better approaches for its diagnosis and treatment.

It is known that disturbances in amino acid metabolism play a role in an inadequate bone healing response, especially amino acids related to the arginine-nitric oxide metabolism ${ }^{10}$. Nitric oxide (NO, solely produced during conversion of arginine into citrulline by one of the nitric oxide synthases, NOSs) production is important during fracture healing because of its influence on stimulation of bone cells to regulate bone remodelling ${ }^{11}$, vascularization ${ }^{12}$ and a possible stimulation of polyamine production as a precursor for collagen synthesis 13,14 . In vivo animal studies already showed a localized ${ }^{15}$ and temporal ${ }^{16}$ expression of the different NOS isoforms in fracture tissue. Next to this, absence of NOS isoforms results in diminished bone formation and nonunion development ${ }^{17}$. In humans, NOS isoforms were also expressed in mRNA in callus samples ${ }^{18}$, indicating an active role in the healing process.

We hypothesize that the arginine-NO metabolism in the human body plays a role in the molecular pathogenesis of abnormal bone healing and that measuring the concentrations of these amino acids and its related enzymes from the bone harvested during RIA procedure is indicative of the success or failure of the non-union treatment.

Hence, here we investigate amino acid concentrations and relevant enzyme expression in bone marrow obtained during the RIA procedure for autologous bone grafting in long bone non-unions, and comparing it between successful outcome and failure of this treatment. Patients with comparable fractures with normal fracture healing after fracture treatment were included as baseline control samples.

\subsection{PATIENTS \& METHODS}

\subsubsection{Patient inclusion}

This study was approved by the medical ethics committee of the Maastricht University Medical Center (permit METC04021). Written informed consent was obtained from all patients. Included were patients which were admitted for surgery on atrophic diaphyseal long bone non-unions at the Department of Surgery at the Maastricht University Medical Center and were the reamer-irrigator-aspirator (RIA) procedure was performed. Atrophic non-union was defined as a fracture which has, by opinion by the treating physician, no chance of healing without any further intervention. 17 patients were included and retrospectively divided into two groups. In the first group 9 patients achieved primary healing of the RIA procedure (bone healing within 6-9 


\section{CHAPTER 6 |}

months after surgery), and 8 patients in the second group either had secondary success (one or more re-interventions after the primary RIA procedure to obtain healing of the non-union) or persisting absence of healing. As control tissue, trabecular bone samples were obtained during regular elective surgery in which there was access to the bone marrow in patients with healed femur or tibia fractures in order to remove the osteosynthesis materials. Patient characteristics are presented in table 6.1. The nonunion scoring system (NUSS) was used to classify the non-union severity.

Bone marrow samples were collected directly after harvesting the marrow and snap frozen in liquid nitrogen in the operating theatre. Samples were stored at $-80{ }^{\circ} \mathrm{C}$ until analysis.

Table 6.1. Patient characteristics. (BMI: body mass index, NSAID: non-steroidal anti-inflammatory drugs, NUSS: non-union scoring scale, DM: diabetes mellitus, NSAID: non-steroidal inflammatory drugs. Significance $p<0.05$.

\begin{tabular}{|c|c|c|c|c|}
\hline & $\begin{array}{c}\text { Normal bone } \\
\text { healing }\end{array}$ & $\begin{array}{c}\text { Nonunion with } \\
\text { primary success }\end{array}$ & $\begin{array}{c}\text { Refractory nonunion } \\
\text { (Secondary success/failure) }\end{array}$ & Significance \\
\hline Patient nr. & $\mathrm{n}=8$ & $\mathrm{n}=9$ & $\mathrm{n}=8$ & \\
\hline Age (years) & $58(30-66)$ & $64(51-86)$ & $44(18-71)$ & $\begin{array}{c}\mathrm{p}=0.03^{*} \\
\text { (prim vs sec.) }\end{array}$ \\
\hline Male/female & $4 / 4$ & $3 / 6$ & $5 / 3$ & ns \\
\hline Length (cm) & $174(166-191)$ & $172(160-187)$ & $176(165-192)$ & ns \\
\hline Weight (kg) & $80(65-94)$ & $83(60-108)$ & $81(62-108)$ & ns \\
\hline BMI $\left(\mathrm{kg} / \mathrm{cm}^{2}\right)$ & $20.0(19.1-30.4)$ & $28.0(22.9-31.2)$ & $26.5(19.0-39.7)$ & $\begin{array}{c}\mathrm{p}<0.05^{*} \\
\text { (control vs. both } \\
\text { non-union } \\
\text { groups) }\end{array}$ \\
\hline Alcohol use (yes/no) & $3 / 5$ & $5 / 4$ & $1 / 7$ & ns \\
\hline Smoking (yes/no) & $6 / 2$ & $5 / 4$ & $6 / 2$ & ns \\
\hline NSAID use (yes/no) & $2 / 6$ & $1 / 8$ & $2 / 6$ & ns \\
\hline DM (yes/no) & $2 / 6$ & $2 / 7$ & $3 / 5$ & ns \\
\hline \multicolumn{5}{|l|}{ Localization (n) } \\
\hline Femur & 4 & 2 & 5 & \\
\hline Tibia & 4 & 3 & 1 & \\
\hline Humerus & 0 & 3 & 2 & \\
\hline Radius & 0 & 1 & 0 & \\
\hline Defect size (mm) & $\mathrm{n} / \mathrm{a}$ & $30.4(6-84)$ & $50.4(8-155)$ & ns \\
\hline \multicolumn{5}{|l|}{ Gustillo (n) } \\
\hline $\mathbf{0}$ & 8 & 5 & 2 & \\
\hline 1 & 0 & 2 & 1 & \\
\hline 2 & 0 & 0 & 1 & \\
\hline 3 & 0 & 2 & 4 & \\
\hline NUSS score $(0-100)$ & $\mathrm{n} / \mathrm{a}$ & $64(51-86)$ & $44(18-71)$ & $\begin{array}{c}\mathrm{p}=0.028^{*} \\
\text { (prim vs sec). }\end{array}$ \\
\hline Time fracture to sampling (days) & $2(1-4)$ & $499(65-1143)$ & $655(191-2331)$ & ns \\
\hline
\end{tabular}




\subsubsection{High-performance liquid chromatography amino acid analysis}

For measurement of relevant amino acids concentrations, tissue samples were crushed on liquid nitrogen, deproteinized, homogenized and centrifuged as described before in detail ${ }^{17}$. The obtained supernatant was 100 -fold diluted in water and $100 \mu \mathrm{l}$ was placed in a WISP-style vial and placed in the chilled (4-8 ${ }^{\circ} \mathrm{C}$ ) sample compartment from a Waters 717 plus Autosampler (Waters Chromatography BV, Etten-Leur, The Netherlands). The amino acid analysis was performed after pre-column derivatization using $o$-phthaldialdehyde (Thermo Fisher Scientific) as described previously ${ }^{19}$.

\subsubsection{RNA isolation and qPCR}

Before RNA isolation, samples were crushed with pestle and mortar on liquid nitrogen. To isolate total RNA, crushed samples were incubated, precipitated and centrifuged as described before ${ }^{17}$. Afterwards, pellets were washed and dried before dissolving in diethylpyrocarbonate treated water for subsequent cDNA synthesis.

For quantitative PCR, iQ SYBR Green Supermix (Biorad Products, Hercules, CA, USA) and gene-specific forward and reversed primers were added to the cDNA. The cDNA was amplified using the MyiQ system (Biorad Products, Hercules, CA, USA) via a 3-step program: 40 cycles of denaturation $\left(95^{\circ} \mathrm{C}, 10 \mathrm{~s}\right)$, annealing $\left(60^{\circ} \mathrm{C}, 20 \mathrm{~s}\right)$ and elongation ( $70^{\circ} \mathrm{C}, 20 \mathrm{~s}$ ). Gene expression levels of Nos2, Nos3, and Arg1 were determined using IQ5 software (Biorad Products, Hercules, CA, USA). The geometric mean of cyclophylin A (Ppia) and $\beta$-actin (ActB) expression levels was calculated and used as a normalization factor. All primers were acquired from Sigma-Aldrich (Zwijndrecht, The Netherlands). Primer sequences are depicted in table 6.2.

Table 6.2. Primer sequences for quantitative polymerase chain reaction. $(\mathrm{Fw}=$ forward, $\mathrm{Rev}=$ reverse $)$

\begin{tabular}{|c|c|c|}
\hline Gene & Name & Sequence $\left(5^{\prime} \rightarrow 3^{\prime}\right)$ \\
\hline \multirow{2}{*}{ Ppia } & Cyclophylin-A (Fw) & CTCGAATAAGTTTGACTTGTGTTT \\
\hline & Cyclophylin-A (Rev) & CTAGGCATGGGAGGGAACA \\
\hline \multirow{2}{*}{ ActB } & Beta-actin (Fw) & GCTGTGCTACGTCGCCCTG \\
\hline & Beta-actin (Rev) & GGAGGAGCTGGAAGCAGCC \\
\hline \multirow{2}{*}{ Nos2 } & iNOS (Fw) & TTGCAAGCTGATGGTCAAGATC \\
\hline & iNOS (Rev) & CAACCCGAGCTCCTGGAA \\
\hline \multirow{2}{*}{ Nos3 } & eNOS (Fw) & TTAATGTGGCCGTGTTGCA \\
\hline & eNOS (Rev) & CTCTTGATGGAAGACAGGAGTTAGG \\
\hline \multirow{2}{*}{$\operatorname{Arg} 1$} & Arginase-1 (Fw) & CGCCAAGTCCAGAACCATAGG \\
\hline & Arginase-1 (Rev) & TCTCAATACTGTAGGGCСТTCTT \\
\hline
\end{tabular}




\subsubsection{Statistical analysis}

Statistical analyses were performed using GraphPad Prism 6 (GraphPad, San Diego, CA, USA). Normality was checked using the Shapiro-Wilk test. All data are presented as means and standard error of the mean (SEM). Significance was calculated using oneway ANOVA testing with post hoc Bonferroni correction. P-values below 0.05 were considered as statistical significant. For regression analysis, SPSS 25.0 was used (IBM, Armonk, NY, USA). A multivariate procedure was used to provide a regression analysis and analysis of variance for dependent variable group and with the factors BMI, age, NUSS and the arginine-metabolism related factors Nos- and Arginase 1-expression, and concentrations of ARG, CIT and ORN as covariates, presented as non-standardized regression coefficients (B) (with $\mathrm{SE}$ ).

\subsection{RESULTS}

\subsubsection{Patient characteristics}

Demographic characteristics of patients included within this study are presented in table 1. A significant age differences was observed between patients with primary success and patients with refractory non-union $(\mathrm{p}<0.05)$. Although length and weight of the patients did not show significant differences, BMI from patients in the control group was significantly lower in comparison with both the primary and secondary success after RIA treatment groups (both $\mathrm{p}<0.05$ ). The NUSS score was significantly higher is patients with primary success after treatment when compared with refractory non-union patients. All other demographic factors (sex, smoking, alcohol and NSAID use, history of diabetes and the fracture location and Gustilo grade did not show any significant differences between the groups.

\subsubsection{Amino acid concentrations}

In Figure 6.1. concentrations of arginine, citrulline and ornithine measured in reamed intramedullary aspirate are shown.

Arginine concentrations are significantly higher in patients in the primary success group when compared to patients with failure of the RIA treatment which needed one or more re-interventions ( $p<0.05$, respectively $225 \pm 46.9$ and $113 \pm 17.2 \mu \mathrm{mol} / \mathrm{mg}$ wet tissue, Figure 6.1.A). No significant differences were found when concentrations in both patient groups treated with RIA were compared with control samples.

Citrulline concentrations in samples of primary success patients $(173 \pm 50.3 \mu \mathrm{mol} / \mathrm{mg}$ wet tissue) and the secondary success or failure group (109 \pm 26.7$)$ were comparable. However, patients which achieved primary success of the RIA procedure showed significant higher citrulline levels when compared to the control group $(45.3 \pm 3.49$, p $<0.05$, Figure 6.1.B), while patients with a secondary success or failure had similar concentrations compared to control patients.

Ornithine concentrations also showed different concentrations between the three study groups (Figure 6.1.C). Samples obtained from control patients showed significant higher ornithine concentrations $(148 \pm 7.38 \mu \mathrm{mol} / \mathrm{mg}$ wet tissue $)$ when compared with primary success samples $(102 \pm 7.57, \mathrm{p}<0.001)$ as well as patients with failure of healing after RIA $(71.5 \pm 7.69, \mathrm{p}<0.0001)$. Next to this, patients with initial success of the RIA procedure also showed higher ornithine levels when compared to patients with failure of treatment or with the need of a secondary surgical intervention $(p<0.05)$. 


\subsection{2. qPCR analysis}

RNA-expression of enzymes relevant to the arginine-NO metabolism is shown in figure 6.2. Measurements of Nos2 (inducible nitric oxide synthase) expression in trabecular bone from the reamed intramedullary aspirate showed a significant upregulation in patients with a successful RIA procedure as well as in patients where the procedure was not successful (both $\mathrm{p}<0.05$ ) when compared to samples obtained from control patients (Figure 6.2.A). Nos3, the enzymatic isoform present in the end othelium of bone vasculature, was not detectable in all patients who were treated with the RIA-procedure (data not shown).
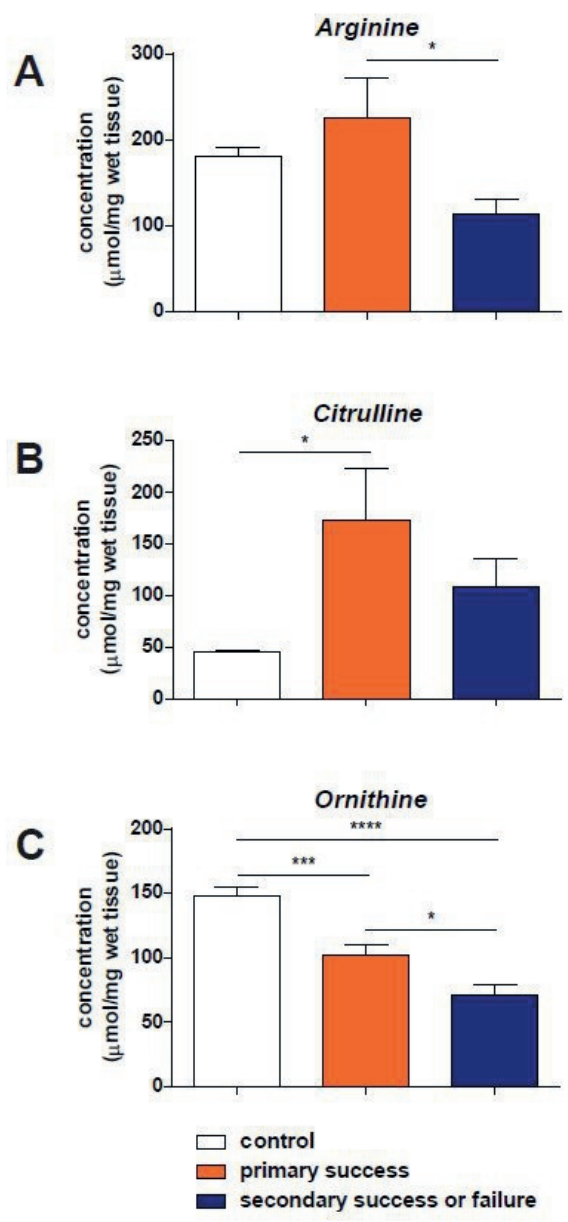

Figure 6.1. Concentrations of amino acids. Concentrations of arginine (6.1.A), citrulline (6.1.B) and ornithine (6.1.C) in reamed intramedullary aspirate, presented as $\mu \mathrm{mol} / \mathrm{mg}$ wet tissue. Results in control tissues are presented in the white bars. Samples obtained from patients with a primary successful RIA treatment are shown in orange and with an unsuccessful treatment in dark blue. Statistical significance: ${ }^{*} \mathrm{p}<$ $0.05,{ }^{* * *} \mathrm{p}<0.001,{ }^{* * * *} \mathrm{p}<0.0001$. 


\section{CHAPTER 6 |}

In Figure 6.2.B, the expression of $A r g 1$ is shown in the three study groups. A significant downregulation ( 0.24 and 0.34 of the values in control patients) is visible in both RIA treated groups of patients when compared to control samples $(\mathrm{p}<0.001$ and $\mathrm{p}<0.05$ for the primary success and the secondary success and failure group respectively).

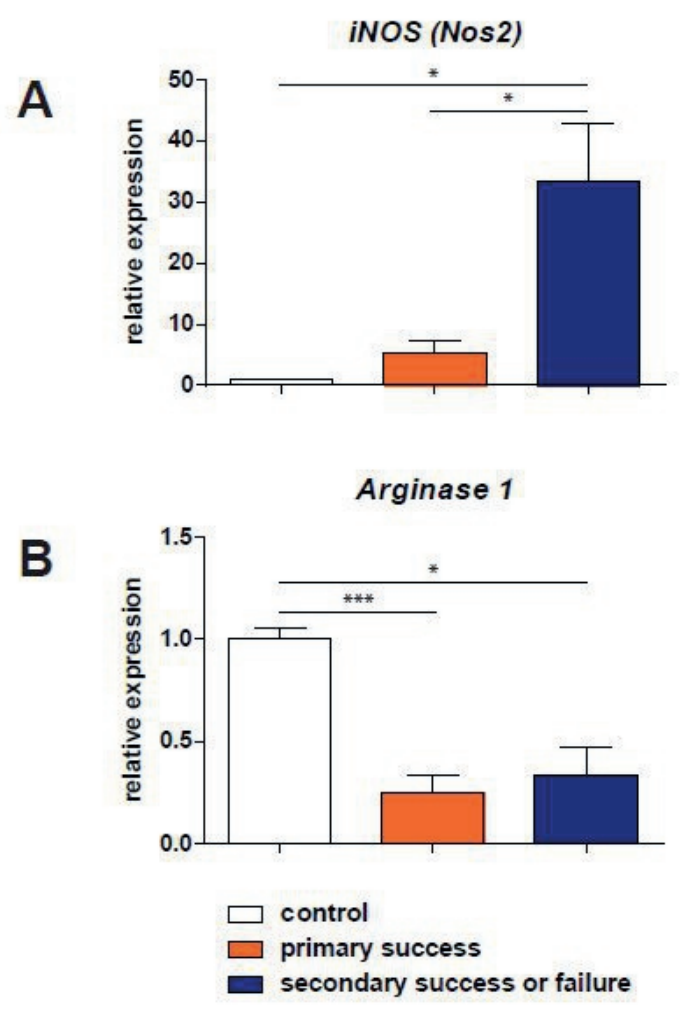

Figure 6.2 Relative expression of enzymes. Relative expression of iNOS (Nos2, inducible nitric oxide synthase, 6.2. A) and Arginase-1 (Arg1, 6.2. B). Results in control tissue are represented in white bars. Samples obtained from patients with subsequent successful RIA treatment are shown in orange. The failure of treatment group is presented in dark blue. Statistical significance: ${ }^{*} \mathrm{p}<0.05,{ }^{* * *} \mathrm{p}<0.001$.

\subsubsection{Regression analysis}

All variables were subsequently used as independent variables in a logistic regression analysis. The dependent variables age, BMI, NUSS, arginine-, citrulline- and ornithine concentrations and arginase- 1 and iNOS expression were included being significant predictors of outcome on univariate analysis. On multivariate analysis, iNOS was the only significant factor within these variables. Below the overview of the different significance levels and non-standardized regression coefficients (with SE): age (B 0.001, SE 0.215, p = 0.514), BMI (B -0.014, SE 0.006, p = 0.057), NUSS (B 0.003, SE 0.002, p = 0.202 ), arginine concentration ( $B-0.001$, SE $0.000, p=0.179$ ), citrulline concentration (B 0.000, SE 0.000, $p=0.357$ ), ornithine concentration (B -0.003, SE 0.002, $p=0.146$ ), 
arginase-1 expression (B 0.421, SE 0.190, p = 0.057) and iNOS expression (B 0.025, SE $0.002, \mathrm{p}<0.001)$.

\subsection{DISCUSSION}

This is the first study in which biomarkers of the arginine-nitric oxide metabolism are clinically evaluated in trabecular bone harvested during RIA from patients treated for non-union of the long bones. Both concentrations of the amino acids arginine and ornithine were higher in samples obtained from patients that had successful bone healing after bone grafting when compared with those that had an unsuccessful healing. Relevant enzymes, Nos2 and Arg1, showed differences in samples obtained from the reamed intramedullary aspirate when compared to bone marrow obtained from patients with an initial normal fracture healing, while Nos2 was able to differentiate between successful and primarily failed non-union treatment. This predictive value might lead towards a possible future use as biomarker in predicting non-union healing outcome.

Generally, $10-15 \%$ of all long-bone fractures fail to heal adequately $1,20,21$ with resulting development of non-unions with major functional impairment and a decrease in the quality of life for these patients, which is accompanied by a high socio-economic burden 2,4. A wide range of possible biomarkers that can be used as a predictor for the development of non-unions have been investigated 22 , however our current study shows, for the first time, the use of possible biomarkers as a predictor for a successful non-union treatment.

One of the major components of the treatment for long bone non-union is autologous bone grafting. Bone grafting is used with proven effectiveness that the transplantation of sufficient cells, scaffold and growth factors from other, non-affected, locations to the non-consolidating bone can stimulate new bone healing. While this effectiveness of the autologous bone grafting in promoting consolidation of non-union is high, it can vary considerably among patients from 80 to $90 \%{ }^{20,23}$. Knowledge on predicting factors is limited, but encompass clinical and biological markers. A number of studies reported either specific (e.g. scaphoid or tibia) or general (e.g. NUSS score) clinical and radiological factors for predicting outcome after treatment of non-unions ${ }^{24-26}$. In addition, Granchi et al shows a decrease in the biochemical bone turnover markers bone-specific alkaline phosphatase and C-terminal propeptide of type I procollagen were observed during treatment failure ${ }^{27}$. The current study adds to the evidence that biomarkers can have prognostic value in the treatment of patients with a non-union in addition to clinical parameters.

Differences in molecular patterns in bone grafts between patients with success and failure at a site distant from the non-union may indicate that systemic molecular pathologies are partly responsible for the failure of non-union treatment and that nonunion is not a purely local metabolic problem. The decreased concentrations of arginine in the non-union callus tissue in a previous study ${ }^{10}$ and in the harvested bone in the patients with a failed response to the bone harvesting treatment seem to be an indication for this hypothesis.

A sufficient formation of nitric oxide (NO), a free radical, influences vascular reactivity 12 and stimulates bone cells to regulate bone remodelling during fracture repair 11. Through subsequent formation of ornithine, it also stimulates the production of 
polyamines which are precursors for collagen synthesis ${ }^{13}$. Previous studies already showed that callus tissue and plasma samples of patients with non-unions have abnormal low concentrations of amino acids arginine, citrulline and ornithine when compared to normal healed and acute fractures ${ }^{10}$. The importance of the NOS isoforms during fracture healing have up till now mainly be investigated in in vivo models of fracture repair 15-18. Callus tissue of femoral fractures in rats showed a different temporal and spatial expression of these isoforms during the healing process. The inducible NOS (Nos2) is present during the first inflammatory reaction after sustaining the fracture and localized along the edge of the periosteal callus 15,18 . Nos3, which is constitutively expressed is mainly present during later phases of fracture healing in cells lining the blood vessels ${ }^{15,18}$. The fact that Nos1 is expressed during the remodelling stages ${ }^{16}$ led us to not focus on this enzyme during the present study. The correlation of NOSs and fracture repair is further emphasized by experiments in which (non)selective NOS inhibitors are supplemented to animals after inducing a fracture leads to a decrease of cross-sectional callus area ${ }^{18}$. Furthermore, genetic deletion of NOS2 or NOS3 leads to a decreased bone formation and subsequent non-union formation in mice 17,28 .

In the current study, we found significantly increased Nos2 expression in RIA tissue obtained during the RIA procedure in patients were the bone grafting procedure had an unsuccessful outcome, when compared to patients with adequate bone healing after the RIA-procedure. An increased Nos2 expression suggests a prolonged inflammatory response (i.e. stimulation by $\mathrm{NF}-\kappa \mathrm{B}$ ) resulting in the production of proinflammatory cytokines as IL-1, TNF- $\alpha$ and IFN- $\gamma$. Since a disturbed chronic inflammatory response during the fracture healing might result in delayed union or non-union formation, this can be the reason that the clinical response to the RIA treatment is inadequate 29,30 . The significantly lower arginine concentrations that coincide with the higher Nos2 expression may indicate depletion of this amino acid by an increased catabolic response of the patient ${ }^{31}$.

Arg1 is the enzyme converting arginine into ornithine subsequently leading to collagen synthesis. RIA procedures resulting in a successful bone healing as well as in unsuccessful healing showed a 3- to 4-fold lower expression of arginase 1 when compared to normal healed fractures. This might reflect the anabolic response of the bone during the healing process which initially was the cause of the non-union development and the need for surgical repair. This is also reflected by the lower ornithine concentrations measured in the reamed intramedullary aspirate in these patients.

A number of factors are known predictors of development of non-union. While the NUSS score is a known factor in patients with a fracture to define the risk of subsequently developing a non-union, this study found the NUSS score also to be a predictor of the success rate of treatment for the non-union. Interestingly, compared to the NUSS score, the activity of the inflammatory response in the grafted material obtained by RIA was an even better predictor of therapy success.

A limitation of this study is the heterogeneity of different characteristics in patients included in the current investigation. Especially defect size, NUSS 24,32 (non-union scoring scale), Gustilo classification and fracture localization show a wide range for a relative low number of patients. Ideally, a large cohort of patients with similar 
characteristics in all groups is needed to minimalize the possible confounding effects, and this study should therefore be regarded a hypothesis generating pilot study to determine whether the underlying heterogeneity in our population influenced the amino acid concentrations. As for the amino acid concentrations and especially arginine, there are several conditions that can alter these concentrations of arginine and related amino acids in plasma, such as diabetes mellitus, inflammation are renal or hepatosplanchnic dysfunctions, due to the compromising function on the availability and conversion of citrulline into arginine ${ }^{33-36}$. In addition, other host factors such as smoking or alcohol use, the use of NSAIDs, could possibly influence fracture healing negatively ${ }^{2}$. As shown in table 1 , these factors did not significantly differ between the control group and both non-union groups, supporting also the clinical relevance of the presented results.

In conclusion, the results presented in the current study indicate an influence of the arginine-nitric oxide metabolism in bone grafts harvested by reamed intramedullary aspirate on the successful outcome of the autologous bone (marrow) grafting as treatment for long bone non-unions. The use of these biomarkers could add to the prediction of outcome in addition to the clinical parameters available.

\subsection{REFERENCES}

1. Mills LA, Aitken SA, Simpson A. The risk of nonunion per fracture: current myths and revised figures from a population of over 4 million adults. Acta orthopaedica 2017; 88(4): 434-9.

2. Bishop JA, Palanca AA, Bellino MJ, Lowenberg DW. Assessment of compromised fracture healing. The Journal of the American Academy of Orthopaedic Surgeons 2012; 20(5): 273-82.

3. Karladani AH, Granhed H, Karrholm J, Styf J. The influence of fracture etiology and type on fracture healing: a review of 104 consecutive tibial shaft fractures. Archives of orthopaedic and trauma surgery 2001; 121(6): 325-8.

4. Kanakaris NK, Giannoudis PV. The health economics of the treatment of long-bone non-unions. Injury 2007; 38 Suppl 2: S77-84.

5. Giannoudis PV, Einhorn TA, Marsh D. Fracture healing: the diamond concept. Injury 2007; 38 Suppl 4: S3-6.

6. Masquelet AC, Begue T. The concept of induced membrane for reconstruction of long bone defects. The Orthopedic clinics of North America 2010; 41(1): 27-37; table of contents.

7. Belthur MV, Conway JD, Jindal G, Ranade A, Herzenberg JE. Bone graft harvest using a new intramedullary system. Clinical orthopaedics and related research 2008; 466(12): 2973-80.

8. Dawson J, Kiner D, Gardner W, 2nd, Swafford R, Nowotarski PJ. The reamer-irrigator-aspirator as a device for harvesting bone graft compared with iliac crest bone graft: union rates and complications. Journal of orthopaedic trauma 2014; 28(10): 584-90.

9. Stafford PR, Norris BL. Reamer-irrigator-aspirator bone graft and bi Masquelet technique for segmental bone defect nonunions: a review of 25 cases. Injury 2010; 41 Suppl 2: S72-7.

10. Wijnands KA, Brink PR, Weijers PH, Dejong CH,
Poeze M. Impaired fracture healing associated with amino acid disturbances. The American journal of clinical nutrition 2012; 95(5): 1270-7.

11. Chae HJ, Park RK, Chung HT, et al. Nitric oxide is a regulator of bone remodelling. The Journal of pharmacy and pharmacology 1997; 49(9): 897-902.

12. Corbett SA, McCarthy ID, Batten J, Hukkanen M, Polak JM, Hughes SP. Nitric oxide mediated vasoreactivity during fracture repair. Clinical orthopaedics and related research 1999; (365): 247-53.

13. Vittur F, Lunazzi G, Moro L, et al. A possible role for polyamines in cartilage in the mechanism of calcification. Biochimica et biophysica acta 1986; 881(1): 38-45.

14. Xia W, Szomor Z, Wang Y, Murrell GA. Nitric oxide enhances collagen synthesis in cultured human tendon cells. Journal of orthopaedic research : official publication of the Orthopaedic Research Society 2006; 24(2): 159-72.

15. Zhu W, Murrell GA, Lin J, Gardiner EM, Diwan AD. Localization of nitric oxide synthases during fracture healing. Journal of bone and mineral research : the official journal of the American Society for Bone and Mineral Research 2002; 17(8): 1470-7.

16. Zhu W, Diwan AD, Lin JH, Murrell GA. Nitric oxide synthase isoforms during fracture healing. Journal of bone and mineral research : the official journal of the American Society for Bone and Mineral Research 2001; 16(3): 535-40.

17. Meesters DM, Neubert S, Wijnands KAP, et al. Deficiency of inducible and endothelial nitric oxide synthase results in diminished bone formation and delayed union and nonunion development. Bone 2016; 83: 111-8.

18. Diwan AD, Wang MX, Jang D, Zhu W, Murrell GA. Nitric oxide modulates fracture healing. Journal of bone and mineral research : the official journal of the American Society for Bone and Mineral Research 2000; 15(2): 34251. 


\section{CHAPTER 6}

19. van Eijk HM, Rooyakkers DR, Deutz NE. Rapid routine determination of amino acids in plasma by highperformance liquid chromatography with a 2-3 microns Spherisorb ODS II column. Journal of chromatography 1993; 620(1): 143-8.

20. Giannoudis PV, Gudipati S, Harwood P, Kanakaris NK. Long bone non-unions treated with the diamond concept: a case series of 64 patients. Injury 2015; 46 Suppl 8: S48-54.

21. Mills LA, Simpson AH. The relative incidence of fracture non-union in the Scottish population (5.17 million): a 5-year epidemiological study. BMJ open 2013; 3(2).

22. Pountos I, Georgouli T, Pneumaticos S, Giannoudis PV. Fracture non-union: Can biomarkers predict outcome? Injury 2013; 44(12): 1725-32.

23. Giannoudis PV, Einhorn TA, Schmidmaier G, Marsh D. The diamond concept--open questions. Injury 2008; 39 Suppl 2: S5-8.

24. Calori GM, Phillips M, Jeetle S, Tagliabue L, Giannoudis PV. Classification of non-union: need for a new scoring system? Injury 2008; 39 Suppl 2: S59-63.

25. Ramamurthy C, Cutler L, Nuttall D, Simison AJ, Trail IA, Stanley JK. The factors affecting outcome after nonvascular bone grafting and internal fixation for nonunion of the scaphoid. The Journal of bone and joint surgery British volume 2007; 89(5): 627-32.

26. Zura R, Della Rocca GJ, Mehta S, et al. Treatment of chronic ( $>1$ year) fracture nonunion: heal rate in a cohort of 767 patients treated with low-intensity pulsed ultrasound (LIPUS). Injury 2015; 46(10): 2036-41.

27. Granchi D, Gomez-Barrena E, Rojewski M, et al. Changes of Bone Turnover Markers in Long Bone Nonunions Treated with a Regenerative Approach. Stem cells international 2017; 2017: 3674045.

28. Baldik Y, Diwan AD, Appleyard RC, Fang ZM, Wang Y, Murrell GA. Deletion of iNOS gene impairs mouse fracture healing. Bone 2005; 37(1): 32-6.

29. Claes L, Recknagel S, Ignatius A. Fracture healing under healthy and inflammatory conditions. Nature reviews Rheumatology 2012; 8(3): 133-43.

30. Schindeler A, McDonald MM, Bokko P, Little DG. Bone remodeling during fracture repair: The cellular picture. Seminars in cell \& developmental biology 2008; 19(5): 459-66.

31. Wijnands KA, Hoeksema MA, Meesters DM, et al. Arginase-1 deficiency regulates arginine concentrations and NOS2-mediated NO production during endotoxemia. PloS one 2014; 9(1): e86135.

32. Calori GM, Colombo M, Mazza EL, et al. Validation of the Non-Union Scoring System in 300 long bone nonunions. Injury 2014; 45 Suppl 6: S93-7.

33. Flynn NE, Meininger CJ, Haynes TE, Wu G. The metabolic basis of arginine nutrition and pharmacotherapy. Biomedicine \& pharmacotherapy $=$ Biomedecine \& pharmacotherapie 2002; 56(9): 427-38.

34. Moinard C, Cynober L. Citrulline: a new player in the control of nitrogen homeostasis. The Journal of nutrition 2007; 137(6 Suppl 2): 1621S-5S.

35. Windmueller HG, Spaeth AE. Source and fate of circulating citrulline. The American journal of physiology 1981; 241(6): E473-80.
36. Wu G, Morris SM, Jr. Arginine metabolism: nitric oxide and beyond. The Biochemical journal 1998; 336 (Pt 1): 1-17. 


\title{
The impact of plasma-derived micro-vesicles from a femoral fracture animal model on osteoblast function.
}

Shock

2020 Jan;53(1):78-87. doi: 10.1097/SHK.0000000000001336. PMID: 31157718.

\author{
Zhi Qiao ${ }^{1}$ \\ Weikang Wang 1 \\ Peng Luo ${ }^{1}$ \\ Martijn Hofman ${ }^{1}$ \\ Klemens Horst ${ }^{1}$ \\ Gerhard Müller-Newen² \\ Johannes Greven ${ }^{1}$ \\ Frank Hildebrand ${ }^{1}$
}

1 Department of Orthopaedic Trauma and Reconstructive Surgery, University Medical Center RWTH Aachen, Germany.

2 Institute of Biochemistry and Molecular Biology, RWTH Aachen University, Germany. 


\section{ABSTRACT}

The role of micro-vesicles (MVs) in transcellular signal transduction has been demonstrated in different studies. However, the potential modulatory role of MVs in fracture healing remains unclear. Therefore, we investigated the impact of plasmaderived MVs after a femoral fracture on cranial osteoblasts.

A femoral fracture with intramedullary stabilization was induced in Sprague Dawley rats. The animals were sacrificed 3 days (group A), 1 week (group B), or 2 weeks (group C) after trauma induction. Animals without trauma served as controls. Osteoblasts from the cranial bone of a neonatal Sprague Dawley rats were cultured and stimulated with either plasma-derived MVs or MV-free plasma of groups A-C. The effects of MVs on osteoblasts were analysed by growth assay, metabolic assay and quantitative real-time polymerase chain reaction (qRT-PCR) for osteocalcin, RUNX2 and collagen 1A to test differentiation of osteoblasts.

MVs were time-dependently incorporated in osteoblasts and localized mainly around the nucleus. MVs increased the viability of osteoblasts, particularly in the late phase after femoral fracture (group A: $1.22 \pm 0.09$ absorbance (OD 405nM), $\mathrm{p}=$ 0.0276; group B: $1.30 \pm 0.12$ absorbance (OD 405nM), $p=0.0295$; group C $1.50 \pm$ 0.22 absorbance (OD 405nM), $\mathrm{p}=0.0407$ ) compared to the control group (0.98 \pm 0.02 absorbance (OD $405 \mathrm{nM}$ )). Late-phase differentiation of osteoblasts was not stimulated by MVs, but by MV-free plasma (Osteocalcin, groups C vs. control, $\mathrm{p}=$ 0.0454 ). The levels of transforming growth factor $\beta 1$ (TGF- $\beta 1$ ) and insulin-like growth factor 1 (IGF-1) were significantly higher in MV-free plasma than in MVs.

MVs seem to modulate the viability of osteoblasts but not to affect osteoblast differentiation. Further studies are warranted to determine the characteristics and interactions of MVs. Potentially, MVs might act as a diagnostic or therapeutic tool in cases of impairment of fracture healing. 


\subsection{INTRODUCTION}

Micro-vesicles (MV) represent a subset of extracellular vesicles secreted by blebbing and shedding from plasma membranes. They are thought to play a significant role in transcellular signal transduction ${ }^{1,2}$.

MVs contain diverse signalling factors (e.g., miRNAs, surface molecules, and proteins) and are of major importance in cell-to-cell communication ${ }^{2}$. Different stimuli, such as cytokines, bacterial lipopolysaccharides, reactive oxygen species, thrombin, and C-reactive protein, result in significant changes in both the secretion patterns and contents of MVs ${ }^{3}$. These alterations can result in a fundamental change in MV-related signalling transduction ${ }^{4}$. Moreover, MVs selectively bind to cells via specific surface molecules rather than non-specifically interacting with various cell types ${ }^{2,5}$.

Due to the diversity of cell types (e.g., platelets, macrophages, endothelial cells, osteoblasts, and osteoclasts) involved in the complex process of fracture healing, synergistic interactions between these cells are essential. MVs are thought to play an important role in intercellular communication of the above-mentioned cells ${ }^{6-8}$. For example, in vitro studies demonstrated that MVs originating from either endothelial cells or mesenchymal stem cells (MSCs) had the potential to stimulate angiogenesis 9,10. In addition, studies showed that MVs secreted by osteoblasts contained the receptor activator of the nuclear factor-kappa B (NF- $\kappa B$ ) ligand (RANKL), which is known to be involved in the regulation of osteoclast function 11. Furthermore, isolated MVs from bone marrow were shown to have the potential to stimulate the differentiation of osteoblasts 12 .

Despite the aforementioned findings, knowledge about the role of MVs during fracture healing remains sparse, especially the effects of systemically derived MVs on fracture healing. Therefore, in this study, we aimed to characterize the pattern of systemic release of MVs after induction of a femoral fracture and throughout the subsequent healing process. Furthermore, whether these MVs influence function of osteoblasts was investigated.

\subsection{MATERIALS AND METHODS}

\subsubsection{General aspects}

This study was approved by the North Rhine-Westphalian State Agency for Nature, Environment and Consumer Protection (Reg. no.: 84-02.04.2015.A078). All the experiments were performed in accordance with "Guide for the Care and Use of Laboratory Animals" by the National Research Council (US) Committee for the Update of the Guide for the Care and Use of Laboratory Animals. In total, 24 female Sprague Dawley (SD) rats aged 8-10 weeks were used. Due to the protective effects of female hormones in cases of inflammatory stimuli, all the animals were in the same phase of the oestrous cycle as determined by vaginal swabs. An overview of the study protocol is provided in figure 7.1.

\subsubsection{Study groups}

The rats were randomly allocated into a control group and three treatment groups (group A, B, and C; 6 animals/group). The animals in group A were sacrificed 3 days 
post-fracture, whereas those in groups B and C were sacrificed 1 week and 2 weeks post-fracture, respectively.

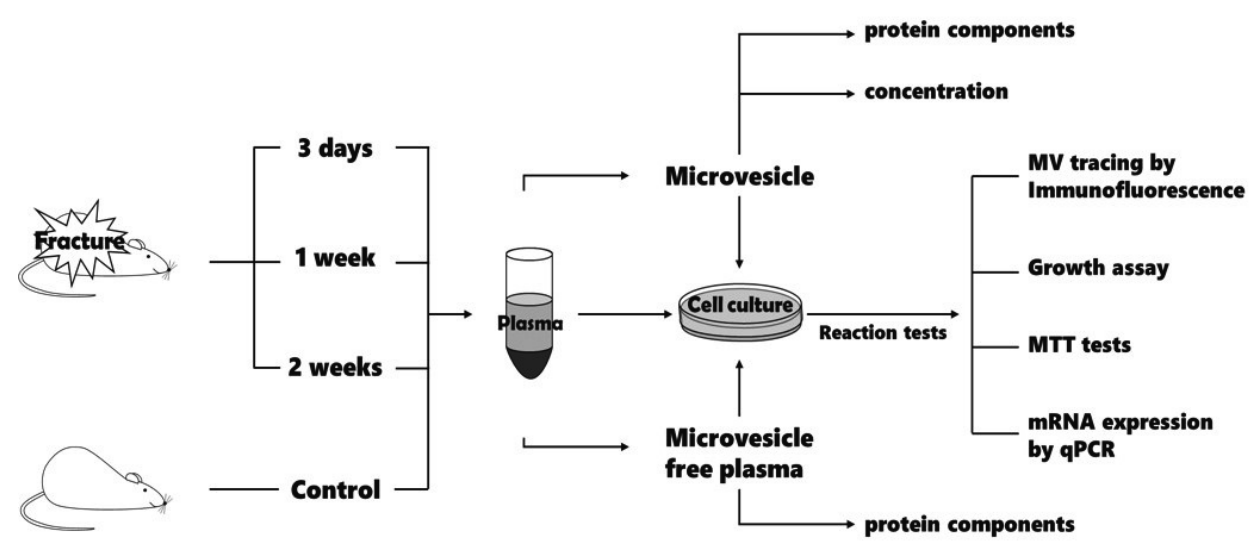

Figure 7.1. Flow chart of the study. The animals were divided into four groups, with six animals in each group. They were randomly allocated into three treatment study groups (A, sacrificed after $3 \mathrm{~d}$ ), (B, sacrificed after 1 week), and (C, sacrificed after 2 weeks) and a control group. Animals from study groups underwent femur fracture and fixation of right hind limbs under anaesthesia. Blood was taken from the animals by heart puncture after sacrifice. The micro-vesicle (MV) concentration within plasma was tested by nanoparticle tracking analysis. Then, MVs were isolated from plasma by centrifugation, and the TGF$\beta 1$ and IGF-1 of both MV and MV-free plasma were tested by an enzyme-linked immunosorbent assay. To test the influence of the MVs from the animal model, primary osteoblasts were isolated from neonatal cultured with culture medium prepared from MV-containing plasma, MVs, and MV-free plasma respectively. The reactions of the osteoblasts to the MVs were tested by a growth assay, 3(4,5Dimethylthiazol-2-yl)-2,5-diphenyltetrazolium bromide (MTT) tests, and quantitative real-timepolymerase chain reaction (qRT-PCR).

\subsubsection{Animal fracture model}

The femur fracture model has been previously described 13 and was applied with minor changes. Briefly, the rats were anesthetized with an intraperitoneal (i.p.) injection of ketamine $100 \mathrm{mg} / \mathrm{kg}$ i.p. (Pfizer, New York, USA) and xylazine $2 \%$ $10 \mathrm{mg} / \mathrm{kg}$ i.p (Xylapan, Vetoquinol, Ravensburg, Germany) before operation. Analgesia was induced with an additional subcutaneous (s.c.) application of buprenorphine hydrochloride $(0.03-0.05 \mathrm{mg} / \mathrm{kg}$ ) (Reckitt Benckiser Healthcare Ltd., U.K.). Afterwards, isoflurane ( $2-2.5 \%$ by volume) was also given consistently via a nasal mask during operation. The adequate anaesthesia was assured by toe pinch reflex test. After anaesthesia, a Kirschner wire $(1.4 \times 26 \mathrm{~mm})$ was inserted intramedullary in the right hind limb femur in treatment groups A, B, and C. A fracture of the femoral shaft was then induced by a $1 \mathrm{~kg}$ plumb-cuboid blunt guillotine, which was dropped from a height of $20 \mathrm{~cm}$. Fracture induction and the correct position of the Kirschner wire were confirmed by X-rays (figure 7.2. A). Buprenorphine hydrochloride $(0.03 \mathrm{mg} / \mathrm{kg}$ s.c. every $6-8 \mathrm{~h}$ for $48 \mathrm{~h}$, subsequently every $12 \mathrm{~h}$ for 3 weeks) and Meloxicam (Metacam; Ingelheim, Germany, Boehringer 
Ingelheim $\mathrm{GmbH})(1 \mathrm{ml} / 300 \mathrm{ml}$ into drinking water for 1 week) were given after fracture induction.

At the end of the study period, the animals were sacrificed by cardiac puncture under adequate anaesthesia and analgesia. Blood was collected in ethylenediamine tetraacetic acid embedded tubes (SARSTEDT, Nürnbrecht, Germany) and centrifuged at $1,200 \mathrm{~g}$ for $5 \mathrm{~min}$ at $4^{\circ} \mathrm{C}$. The plasma was then stored at $-80^{\circ} \mathrm{C}$.

\subsubsection{MV counts and sizes}

Analysis of the concentrations and sizes of MVs in plasma was performed by nanoparticle tracking analysis (NanoSight 300; NTA, NanoSight Ltd., Amesbury, U.K.). For the analysis, $100 \mu \mathrm{l}$ of plasma was centrifuged at 5,000 g for $20 \mathrm{~min}$ to remove platelets and apoptotic bodies. The plasma was then diluted to $200 \mu \mathrm{l}$ with phosphate buffered saline (PBS), which was filtered through a $0.2-\mu \mathrm{m}$ sterile syringe filter (Corning, NY, USA). Samples were then analysed by NanoSight 300 in accordance with the manufacturer's instructions.

\subsubsection{MV isolation and MV-free plasma generation}

MV isolation was performed according to a previously reported method ${ }^{14}$. Briefly, the plasma obtained from each animal was centrifuged at 5,000 g for $20 \mathrm{~min}$. The supernatant was then centrifuged again at 17,000 g for $90 \mathrm{~min}$. The supernatant after second centrifuge was collected as MV-free plasma. The pellet (including the MVs) was resuspended in PBS. The MVs were washed a second time, centrifuged at 17,000 $\mathrm{g}$ for $90 \mathrm{~min}$, and dissolved in PBS.

\subsubsection{Transmission electron microscopy of MVs}

Unfixed isolated MVs were allowed to adsorb for $10 \mathrm{~min}$ on formvar-carbon-coated nickel grids (200 mesh), which was glow discharged for 2 min (Maxtaform; Plano, Wetzlar, Germany). After washing twice with Aqua Dest (Science Services GmbH, Munich, Germany), the samples on grids were stained using a drop of $0.5 \%$ uranyl acetate in Aqua Dest. After air drying, the samples were examined using a LEO 906 (Carl Zeiss, Oberkochen, Germany) transmission electron microscope, operating at an acceleration voltage of $60 \mathrm{kV}$.

\subsubsection{Osteoblast isolation, culture, and identification}

Osteoblast isolation (rat cranial bone) and culture were performed from a neonatal SD rat according to a previously described protocol ${ }^{15}$. The culture medium was changed every 3 days until the cells covered around $80 \%$ of the plate. After trypsinization (Gibco; Thermo Scientific), the cells were collected and seeded into culture flasks (Culturestar, Greine rbio-one $\mathrm{GmbH}$, Frickenhausen, Germany). Thirdpassage cells were used for further analyses.

To identify osteoblasts, a bone formation assay was performed (Figure 2B). Isolated osteoblasts were cultured on a six-well plate with $2 \mathrm{mM} \beta$-GP $(\beta$-Glycerol phosphate, St. Louis, Missouri, USA. Sigma Aldrich), $10 \mathrm{nM}$ dexamethasone (Merck Serono $\mathrm{GmbH}$, Darmstadt, Germany), and $50 \mu \mathrm{g} / \mathrm{ml}$ of ascorbate (Sigma Aldrich). The $\mathrm{pH}$ was adjusted to 7.4 , and $50 \%$ of the culture medium was changed every third day. After 14 days, the cells were washed with PBS two more times, fixed in formaldehyde for 15 min, and washed twice with PBS. After air drying, the cells were stained with 
Alizarin Red S (Sigma Aldrich) for $5 \mathrm{~min}$ and washed with 50\% ethanol (AppliChem, Darmstadt, Germany) three times.

\subsubsection{Imaging of fluorescently labelled MVs}

The MVs and the same volume of PBS as a control were incubated with WGA-Alexa Fluor $^{\mathrm{TM}} 594$ solution $\left(0.5 \mu \mathrm{g} / \mathrm{ml}\right.$ Wheat Germ Agglutinin, Alexa Fluor ${ }^{\mathrm{TM}} 594$ conjugate; Thermo Fisher, Waltham, USA) for $10 \mathrm{~min}$, washed twice with DMEM (Dulbecco's Modified Eagle Medium, Thermo Fisher) and after centrifugation at 17,000 g resuspended in DMEM (Dulbecco's Modified Eagle Medium, Thermo Fisher). The osteoblasts were cultured in a 24-well plate on sterilized cover slips. The cells were incubated with the stained MVs for $10 \mathrm{~min}, 30 \mathrm{~min}$, and $1 \mathrm{~h}$. The control plate was cultured for $1 \mathrm{~h}$. Subsequently, the cover slips were washed with PBS, liquid was removed with blotting paper, and mounting medium (SlowFade ${ }^{\mathrm{TM}}$ Diamond Antifade Mountant with DAPI (4',6-diamidino-2-phenylindole); Thermo Fisher) was used to mount the cells and stain the nuclei. The samples were analysed by confocal microscopy (LSM 710; Carl Zeiss Microscopy GmbH, Jena, Germany). A Z-stack was generated, and a 3D image reconstructed using ZEN 2.3 software (Blue edition; Carl Zeiss Microscopy $\mathrm{GmbH}$ ) to identify intracellular MVs.

\subsubsection{Transforming growth factor- $\beta 1$ (TGF- $\beta 1)$ and insulin-like growth factor 1 (IGF-1) analysis in MVs and MV-free plasma}

Commercialized enzyme-linked immunosorbent assay kits were used to determine the concentration of TGF- $\beta 1$ (Boster Biological Technology, CA, USA) and IGF-1 (Cloud Clone Corp., Wuhan, China). MVs were isolated from $100 \mu \mathrm{l}$ plasma and lysed with $100 \mu \mathrm{l}$ peqGOLD TriFast $^{\mathrm{TM}}$ (peqlab, VWR International GmbH, Radnor, Pennsylvania, USA). The MV solution and MV-free plasma was then analysed according to the protocol.

\subsubsection{Experimental culture medium (CM)}

Four kinds of CMs were used, and for the following assays four kinds of culture medium were prepared:

1. Normal culture medium was prepared with DMEM, $100 \mathrm{U} / \mathrm{ml}$ of penicillin, 50 $\mu \mathrm{g} / \mathrm{ml}$ of streptomycin sulphate, and 10\% FBS (Foetal bovine serum, Thermo Fisher);

2. Normal culture medium containing MVs isolated from plasma resuspended in a comparable amount of FBS (MV-contained culture medium, MVCM) was prepared with DMEM, $100 \mathrm{U} / \mathrm{ml}$ of penicillin, $50 \mu \mathrm{g} / \mathrm{ml}$ of streptomycin sulphate, and $10 \%$ FBS and MV;

3. Culture medium containing plasma from groups $\mathrm{A}, \mathrm{B}, \mathrm{C}$ or the control (plasma contained culture medium, PCM) was prepared with DMEM, $100 \mathrm{U} / \mathrm{ml}$ of penicillin, $50 \mu \mathrm{g} / \mathrm{ml}$ of streptomycin sulphate, $5 \%$ plasma from the animals from groups $\mathrm{A}, \mathrm{B}$, and $\mathrm{C}$, and $5 \% \mathrm{FBS}$;

4. MV-free plasma culture medium was prepared with DMEM, $100 \mathrm{U} / \mathrm{ml}$ of penicillin, $50 \mu \mathrm{g} / \mathrm{ml}$ of streptomycin sulphate, 5\% FBS, and 5\% MV-free plasma from the animals in groups $\mathrm{A}, \mathrm{B}$ and $\mathrm{C}$. 


\subsubsection{Growth assay}

Osteoblasts (5,000 per well) were seeded in 24-well plates (TPP, Trasadingen, Switzerland) and cultured with $500 \mu \mathrm{l}$ of PCM. For each test, triple wells were used as repeats. The medium was changed every third day. After 3, 5, and 8 days, the cells were digested with trypsin, and the total number of cells was counted with Neubauer chamber.

\subsubsection{3-(4,5-Dimethylthiazol-2-yl)-2,5-diphenyltetrazolium bromide (MTT) assay}

An MTT assay was performed to evaluate the viability and proliferation of the osteoblasts ${ }^{16}$. For the assay, 10,000 osteoblasts per well were seeded in 96-well plates and cultured in $200 \mu \mathrm{l}$ of four kinds of CMs (1. to 4.) for 4 days. Each CM experiment was repeated three times. On the fourth day, $20 \mu \mathrm{l}$ of $5 \mathrm{mg} / \mathrm{ml}$ of MTT (Sigma Aldrich) were added into each well and incubated for $4 \mathrm{~h}$. The supernatant was then extracted, and $100 \mu \mathrm{l}$ of dimethylsulfoxide (Sigma Aldrich) was added in each well and gently shaken in the dark for $10 \mathrm{~min}$. The optical density value was measured with wavelengths of $490 \mathrm{~nm}$ and $690 \mathrm{~nm}$ (for correction).

\subsubsection{Quantitative real-time polymerase chain reaction (qRT-PCR)}

The osteoblasts were cultured with four kinds of CMs (1. to 4.) for 5 days and then dissolved with peqGOLD TriFast ${ }^{\mathrm{TM}}$. Total RNA was extracted, and cDNA was synthesized with cDNA kits (Thermo Scientific). The qRT-PCR was performed with SYBR Green real-time PCR Master Mix Reagent (Thermo Scientific) on a StepOne ${ }^{\mathrm{TM}}$ Real-Time PCR System (Applied Biosystems, Waltham, Massachusetts, USA). The expression of runt-related transcription factor 2 (RUNX2), osteocalcin, and collagen 1A were analysed. Peptidylprolyl isomerase A (PPIA) was used as the housekeeping gene. The sequences of the primers are listed in table 7.1. The analysis of gene expression was performed using the $2-\Delta \Delta$ CT method (\%PPIA) ${ }^{17}$.

Table 7.1. Primers of qRT-PCR

\begin{tabular}{|c|c|c|}
\hline Primer & Forward primer & Backward primer \\
\hline PPIA & 5'-GTCAACCCCACCGTGTTCTTC-3' & 5'-CCTTTCTCCCCAGTGCTCAG-3' \\
\hline RUNX2 & 5'-TCCCGTTACAACAGTCTCCC-3' & 5'-TATATGGCTGTGTCGGTCCC-3' \\
\hline Osteocalcin & 5'-GACAAGTCCCACACAGCAACT-3' & 5'-GGACATGAAGGCTTTGTCAGA-3' \\
\hline Collagen 1A & 5'-CAATGGTGGCAGCCAGTTTG-3' & 5'-CCAGGTACGCAATGCTGTTCTT-3' \\
\hline
\end{tabular}

(PPIA = peptidylprolyl isomerase A, RUNX2 = runt-related transcription factor 2)

\subsubsection{Statistical analysis}

Excel 2016 (Microsoft, Redmond, Washington) and GraphPad Prism 7.0 (GraphPad Software, La Jolla, USA) were used for data collection and statistical analysis. The 
Kolmogorov-Smirnov test was applied to test for a normal distribution. An analysis of variance, student's t-test, and Kruskal-Wallis test were performed to test significance, and $\mathrm{p}<0.05$ was accepted as denoting statistical significance.

\subsection{RESULTS}

\subsubsection{Animal model}

To obtain plasma from the trauma model, three groups of animals underwent femur fracture and fixation of right hind limbs under anaesthesia. All the animals survived until the end of the group-specific study period. Correct fixation by intramedullary pins was verified by an X-ray. Osteosynthesis resulted in appropriate stabilization of the femoral fracture (Figure 7.2.A). No sign of infections or other complications were observed. Animals were sacrificed after 3 days, 1 week and 2 weeks and blood plasma was collected through heart puncture.
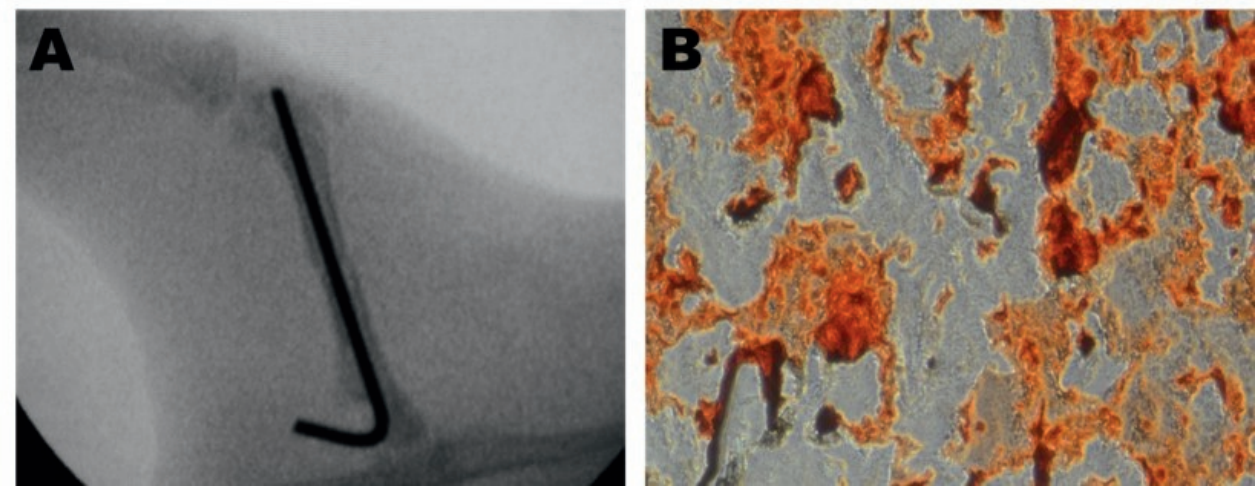

Figure 7.2. Fracture fixation and primary osteoblast isolation. A) Animals from study groups underwent femur fracture and fixation of right hind limb with a Kirschner wire under anaesthesia. A fracture of the right femur shaft and fixation were confirmed by an X-ray. B) Primary osteoblasts were isolated from the cranial bone of a neonatal rat. To certify the osteogenesis of these osteoblasts, a bone formation assay was performed. Osteogenic differentiation was assessed by Alizarin Red S staining, showing the presence of matrix mineralization in orange $(\times 60$ magnification).

\subsubsection{Identification of MVs}

To identify MVs from the animal model, EM (electronic microscopy) was used. Figures 7.3.A and 7.3.B show representative EM images of MVs. Nanoparticle tracking analysis was used to assess size and amount of MVs. Figure 7.3.C shows a typical presentation of MVs obtained by nanoparticle tracking analysis (from 2 weeks group). The average size of the MVs was $185.1 \pm 4.7 \mathrm{~nm}$. A typical size distribution is shown in Figure 3D. There was no significant difference in the MV concentrations of the control and study groups (Figure 7.3.E).

\subsubsection{MV uptake of osteoblasts}

To verify that MVs from the trauma model could be taken up by osteoblasts, fluorescently labelled MVs were co-cultured with primary cranial osteoblasts. Condensed red fluorescence was observed inside the osteoblasts indicating 
incorporation of MVs (Figure 7.4.A). The amount of incorporated MVs increased with the time of incubation. No intracellular red fluorescence was observed in the control group without labelled MVs. The 3D reconstruction of confocal images (Figure 7.4.B) showed that the MVs were located around the nuclei. These results indicated that the cultured osteoblasts were able to incorporate MVs generated under trauma conditions located in the plasma.

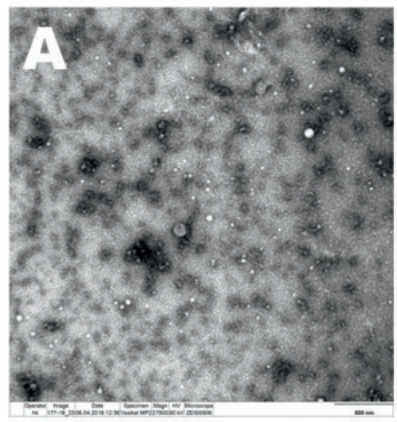

D

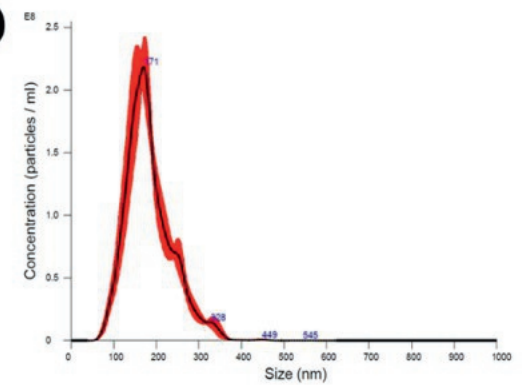

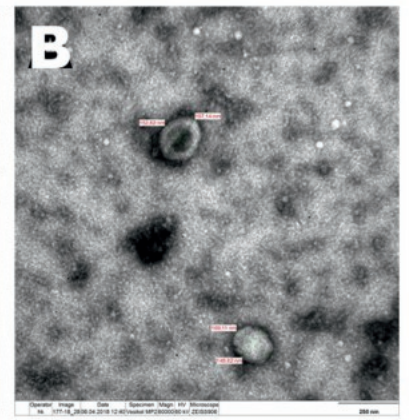

$\mathbf{E}$

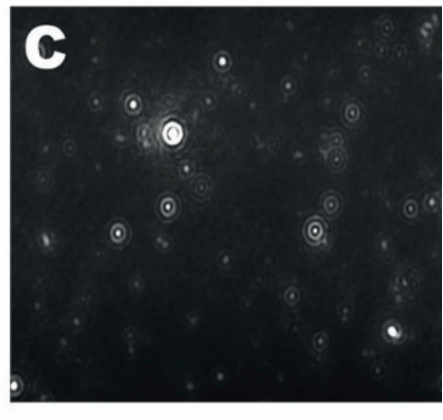

MV counting

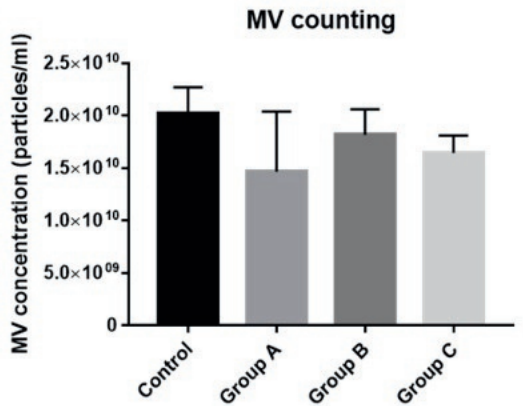

Figure 7.3. Identification of MVs by electronic microscopy and nanoparticle tracking analysis. A and B) To identify the MVs from plasma, a transmission electron microscopy was used (A, 27,800x magnification; B, 60,000x magnification). Arrows denote MVs. The marked MVs shown in B are $152.62 \times$ $197.14 \mathrm{~nm}$ and $169.11 \times 148.62 \mathrm{~nm}$ in size. C, D and E) MVs were analysed with nanoparticle tracking system (NanoSight 300; NTA, NanoSight Ltd.). C shows a typical image of MVs observed by nanoparticle tracking analysis. D shows a typical size distribution of the MVs. No significant differences were found across study groups. E shows the MV count in plasma. There were no significant differences in the MV concentrations among the study groups.

\subsubsection{Concentrations of TGF- $\beta 1$ and IGF-1 in MVs and MV-free plasma}

To locate and quantify osteogenic cytokines in the plasma, concentrations of TGF- $\beta 1$ and IGF-1 were measured with ELISA kits. Compared to the control group (TGF- $\beta 1=$ $64.98 \pm 20.44 \mathrm{pg} / \mathrm{ml} ; \mathrm{IGF}-1=11,107 \pm 885 \mathrm{pg} / \mathrm{ml}), \mathrm{TGF}-\beta 1(24.03 \pm 6.4 \mathrm{pg} / \mathrm{ml})$ and IGF-1 $(6,605 \pm 996 \mathrm{pg} / \mathrm{ml})$ concentrations in MV-free plasma were significantly lower at day 3 (group A) ( $p=0.0320$ and $p=0.0211$, respectively). The levels of TGF$\beta 1$ and IGF-1 within MVs were not significantly different between the control and study groups. In general, the levels of TGF- $\beta 1$ and IGF-1 were significantly higher in 
MV-free plasma than in MVs (Figure 7.5.), which proved that TGF- $\beta 1$ and IGF-1 were mostly located within the MV-free plasma rather than in MVs.

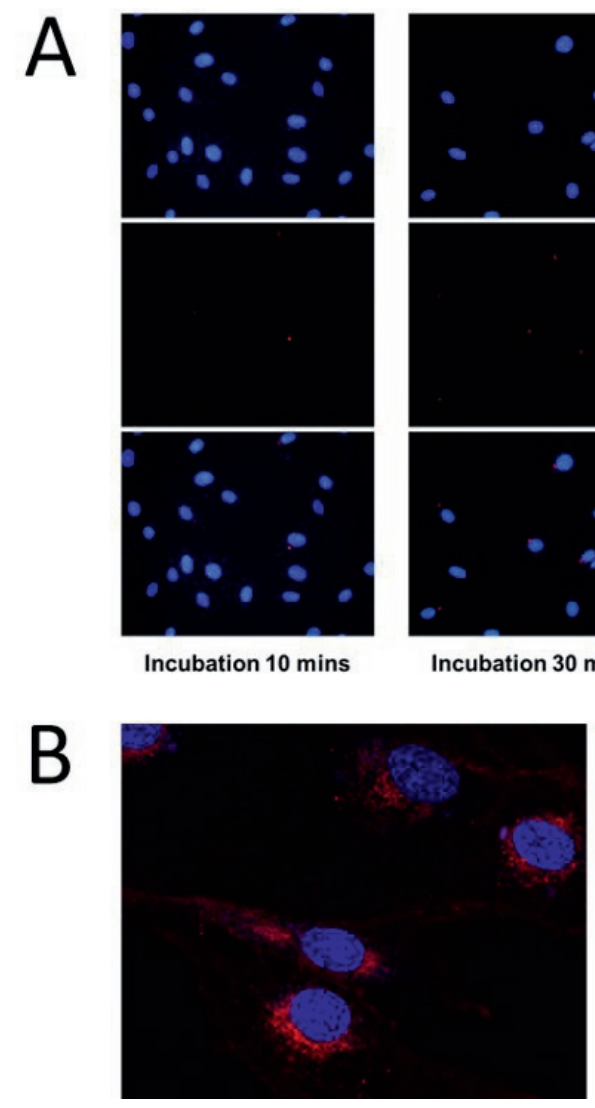

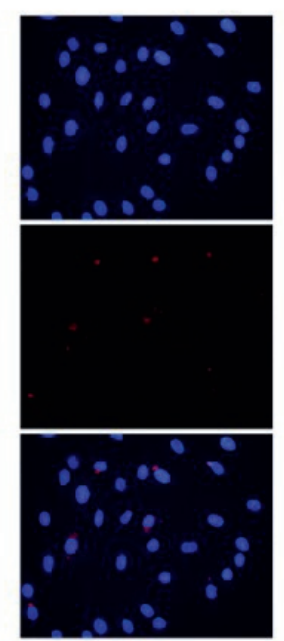

Incubation $1 \mathrm{~h}$
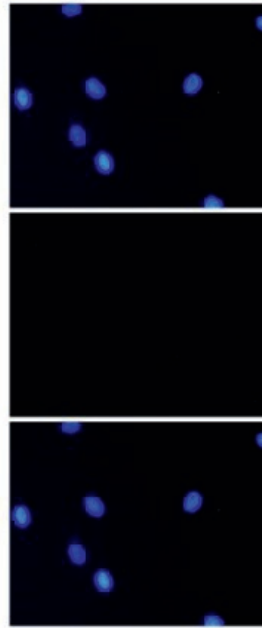

Control

Figure 7.4. Imaging of fluorescently labelled MVs. A) Isolated and fluorescently labelled MVs were cocultured with primary osteoblasts for $10 \mathrm{~min}$., $30 \mathrm{~min}$. and 1 hour. Row 1 shows nuclei of osteoblasts in blue (stained with DAPI). Row 2 shows micro-vesicles (MVs) in red (labelled with WGA-Alexa Fluor ${ }^{\text {TM }}$ 594). Row 3 shows merging of rows 1 and 2 . As the incubation time was increased from $10 \mathrm{~min}$ to $1 \mathrm{~h}$, the MVs accumulated within the cells. No MVs (red fluorescence) were found in the control group. The scale bar represents $50 \mu \mathrm{m}$. B) 3D representation of a z-stack recorded by confocal microscopy. The MVs are enriched around the nuclei. The scale bar represents $10 \mu \mathrm{m}$.

\subsubsection{Growth and differentiation of osteoblasts cultured in PCM}

To assess the influence of full trauma plasma on proliferation and differentiation of primary osteoblasts, osteoblasts were cultured in PCM, made by plasma from study animals. A growth assay was performed, and mRNA expression of osteogenic genes was analysed. As shown in figure 7.6., plasma from group B at day 5 after incubation $\left(3.46 \pm 1.59 \times 10^{4}\right.$ vs. $2.06 \pm 1.31 \times 10^{4}$ cell count, $\left.\mathrm{p}=0.0318\right)$ and from group $\mathrm{C}$ at day 8 after incubation ( $11.31 \pm 4.77 \times 10^{4}$ vs. $4.02 \pm 1.03 \times 10^{4}$ cell count, $\left.p=0.009\right)$, significantly stimulated the growth of osteoblasts comparing with the control group. 
$\mathbf{A}$

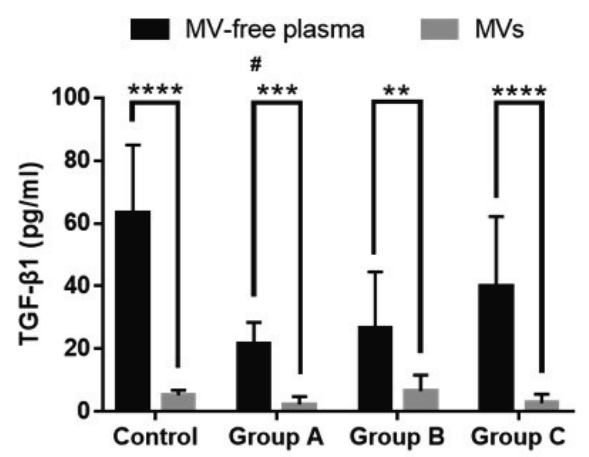

B

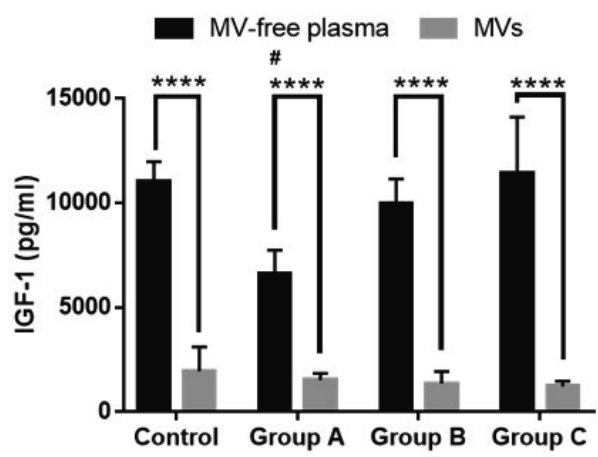

Figure 7.5. Transforming growth factor- $\beta 1$ (TGF- $\beta 1$ ) and insulin-like growth factor 1 (IGF-1) in MVs and in MV-free plasma. MVs from $100 \mu \mathrm{l}$ plasma were lysed with equal amount of peqGOLD TriFast $^{\mathrm{TM}}$. Concentrations of TGF- $\beta 1$ and IGF- 1 were measured with by ELISA. Data from animals without fracture were set as control. In all groups, concentrations of TGF- $\beta 1$ and IGF-1 differed significantly between MV and MV-free plasma. The concentration of TGF- $\beta 1$ in group A (3 days) was significantly decreased compared to the control group ( $24.03 \pm 6.4$ vs. $64.98 \pm 20.44 \mathrm{pg} / \mathrm{ml}, \mathrm{p}=0.032)$. The IGF-1 concentration in group A ( 3 days) was significantly decreased compared to the control group $(6,605 \pm 996$ vs. $11,107 \pm 885 \mathrm{pg} / \mathrm{ml}, \mathrm{p}=0.0211$ ). $\# \mathrm{p}<0.05,{ }^{* *} \mathrm{p}<0.01,{ }^{* * *} \mathrm{p}<0.001,{ }^{* * * *} \mathrm{p}<0.0001$.

\section{Growth assay}

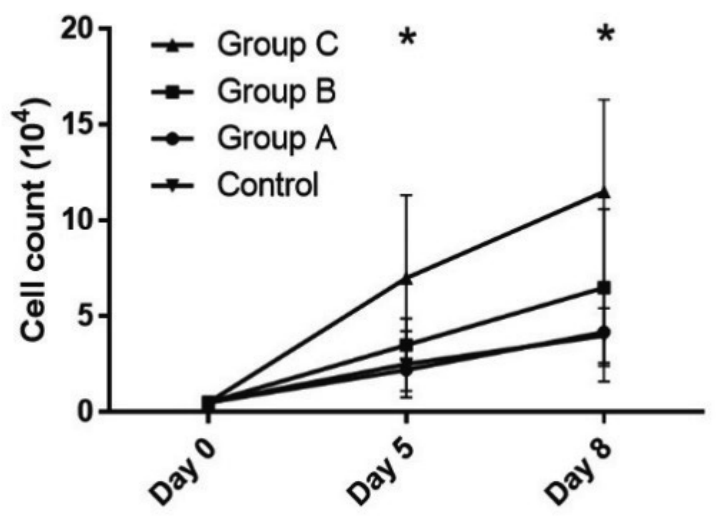

Figure 7.6. Growth assay. Osteoblasts from the study and control groups (non-fracture) were cultured with plasma. Plasma from group B (1 week) significantly stimulated the proliferation of osteoblasts at 5 days of incubation ( $p=0.0318$ ) and plasma from group C (2 weeks) significantly stimulated the proliferation of osteoblasts at 8 days of incubation $(p=0.009)$.

PCM was associated with an increase in osteoblast viability over time, with significantly higher viability in group $\mathrm{C}$ as compared with that in the control group $(1.13 \pm 0.09$ vs. $0.65 \pm 0.12$ absorbance (OD 405nM); $p=0.0393$ ) (Figure 7.7.A). As previously reported, RUNX 2 and collagen $1 \mathrm{~A}$ synthesis served as markers of earlyphase osteogenesis, and osteocalcin synthesis as late-phase osteoblastic differentiation markers ${ }^{18}$. Osteocalcin mRNA expression of osteoblasts was reduced 


\section{CHAPTER 7 |}

in the early post-trauma phase ( $\leq 1$ week) as compared with that in the controls (group B vs. control: $2.07 \pm 2.47$ vs. $7.14 \pm 5.29$ PPIA\%, $p=0.0172$, Figure 7.7.C). In the later phase ( 2 weeks) a significant increase occurred (group B vs. C: $2.07 \pm 2.47$ vs. $10.31 \pm 1.76$ PPIA $\%, p=0.0021$ ). Comparisons of collagen $1 \mathrm{~A}$ and RUNX2 revealed no significant differences in any of the groups (Figure 7.7.B and 7.7.D). These results indicated that, plasma from traumatized animals influences growth and differentiation of the primary osteoblasts.

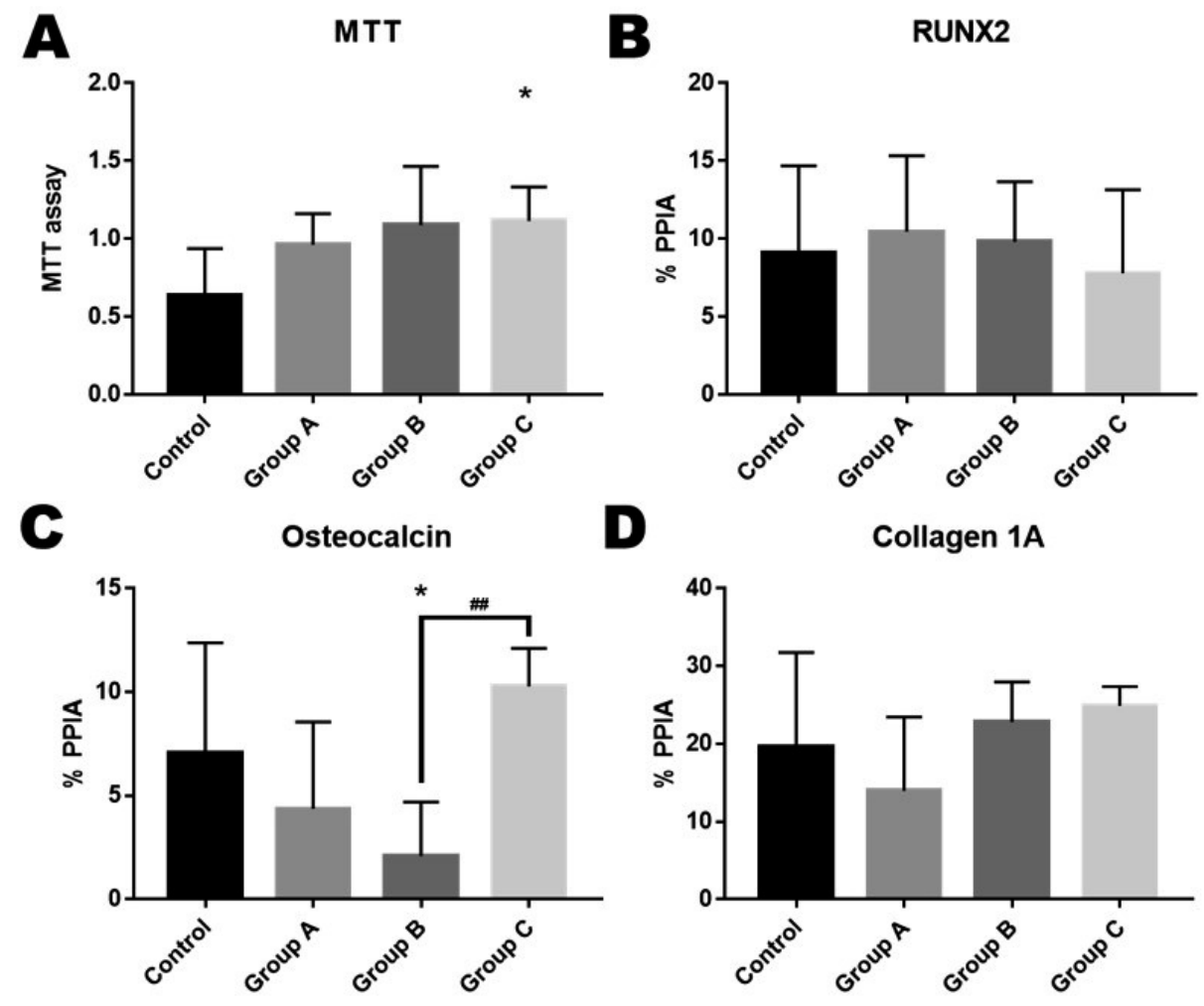

Figure 7.7. Influence of plasma from trauma animals on primary osteoblasts. Primary osteoblasts were cultured with medium containing plasma from fracture model (PCM). Data from animals without fracture were set as control. A) Osteoblast viability was tested by 3-(4,5-Dimethylthiazol-2-yl)-2,5diphenyltetrazolium bromide (MTT). Results showed significantly increased in group C (1.13 \pm 0.09 vs. $0.65 \pm 0.12$ absorbance (OD 405nM); $\mathrm{p}=0.0393$ ). B, C, and D) The osteogenesis mRNA expression of runtrelated transcription factor 2 (RUNX2), osteocalcin, and collagen 1A were measured by qRT-PCR. As compared within osteocalcin, mRNA expression was lower in group B as compared to the control group $(2.07 \pm 2.47$ vs. $7.14 \pm 5.29$ PPIA $\%, p=0.0172)$, and to group C $(2.07 \pm 2.47$ vs. $10.31 \pm 1.76$ PPIA\%, $p=$ $0.0021)$. There was no significant difference in RUNX2 and collagen 1 A expression in any of the groups. $\left(^{*}\right.$ compared with the control group, $\mathrm{p}<0.05$, \#\# $\mathrm{p}<0.01$ ). 

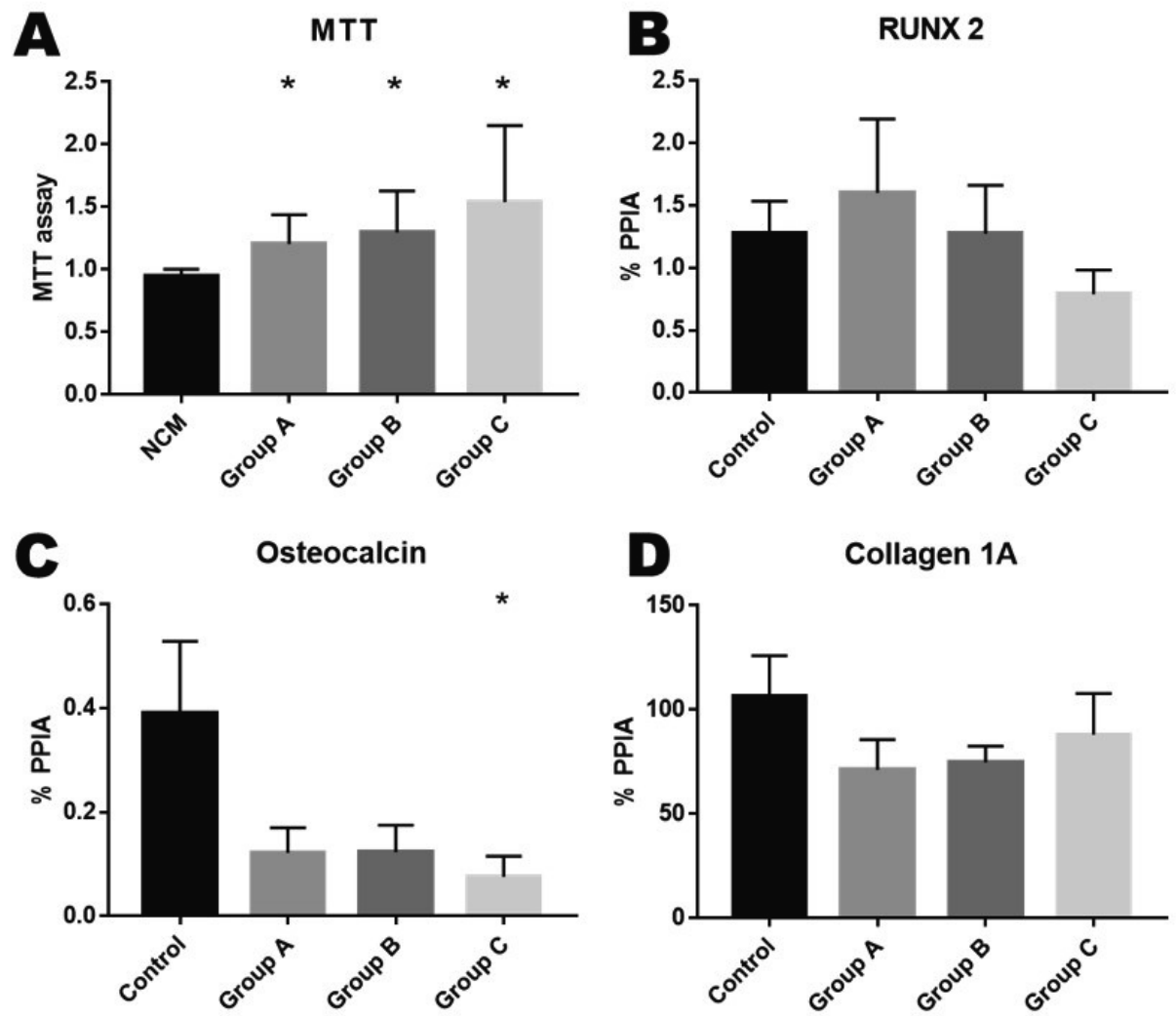

Figure 7.8. Influence of plasma-derived micro-vesicles (MVs) from the fracture model on primary osteoblasts. Primary osteoblasts were cultured with medium containing MVs from fracture model (MVCM). Data from animals without fracture were set as control. A. Viability of osteoblasts was tested by 3-(4,5-Dimethylthiazol-2-yl)-2,5-diphenyltetrazolium bromide (MTT) assay. A) Significant increases in osteoblast viability were observed (group A: $1.22 \pm 0.09$ absorbance (OD $405 \mathrm{nM}$ ), $\mathrm{p}=0.0276$; group B: $1.30 \pm 0.12$ absorbance (OD 405nM), $p=0.0295$; group C $1.50 \pm 0.22$ absorbance (OD 405nM), $\mathrm{p}=0.0407$ ), compared to the control group ( $0.98 \pm 0.02$ absorbance (OD $405 \mathrm{nM})$. B, C, and D) The osteogenesis mRNA expression of runt related transcription factor 2 (RUNX2), osteocalcin, and collagen $1 \mathrm{~A}$ were measured by qRT-PCR. Only osteocalcin mRNA expression was significantly reduced in group C $(0.07 \pm 0.04$ PPIA $\%$, p= $0.0395)$ as compared with that in control $(0.39 \pm 0.14$ PPIA\%). There was no significant difference between groups in RUNX2 and collagen $1 \mathrm{~A}$ expression. $\left({ }^{*} \mathrm{p}<0.05\right)$.

\subsubsection{Viability/proliferation and differentiation of osteoblasts cultured in MVCM}

The functions of MVs from trauma plasma on primarily cultured osteoblasts were assessed. MVs from trauma and control plasma were isolated, and co-cultured with the primary osteoblasts in culture medium made from FBS (MVCM). As depicted in figure 7.8.A, stimulation with MVs resulted in a significant increase in osteoblast viability in all the study groups (group A: $1.22 \pm 0.09$ absorbance (OD $405 \mathrm{nM}$ ), $\mathrm{p}=$ 0.0276; group B: $1.30 \pm 0.12$ absorbance (OD $405 \mathrm{nM}$ ), $\mathrm{p}=0.0295$; group C $1.50 \pm$ 
0.22 absorbance (OD 405nM), $\mathrm{p}=0.0407)$ compared to the control group $(0.98 \pm$ 0.02 absorbance (OD $405 \mathrm{nM})$ ). Osteocalcin was significantly reduced in group $\mathrm{C}$ compared to the control group $(0.07 \pm 0.04$ vs. $0.39 \pm 0.14$ PPIA $\%, p=0.0395)$ (Figure 7.8. C). There expression of osteocalcin was also decreased in group A and B ( $p=$ $0.0686, p=0.0708$ ), however they did not show statistical significance. Analysis of RUNX 2 and collagen $1 \mathrm{~A}$ revealed no significant differences between the control and study groups (Figure 7.8.B and 7.8.D). These results showed the ability of MVs from trauma plasma to influence viability and differentiation of osteoblasts.
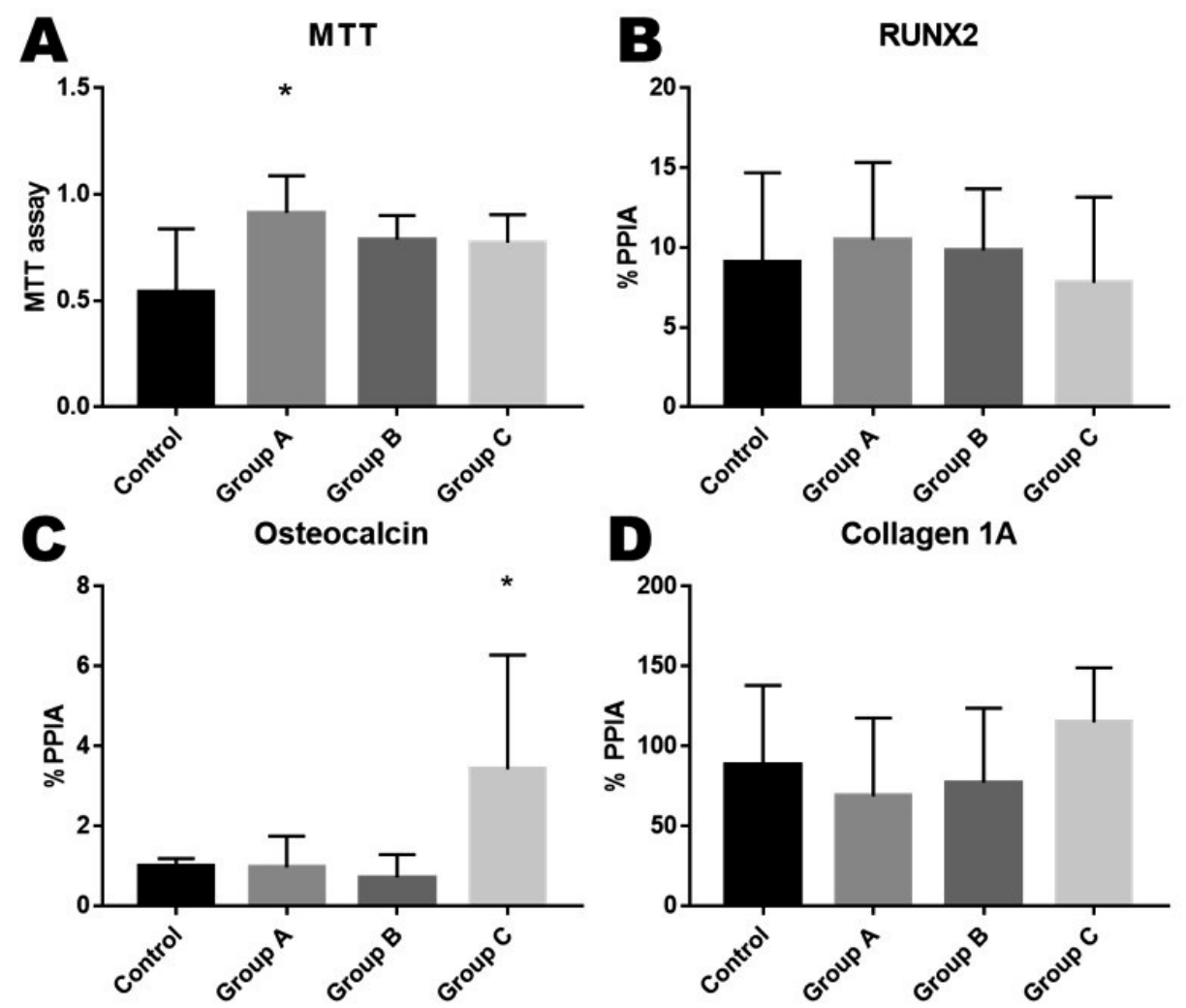

Figure 7.9. Influence of micro-vesicle (MV)-free plasma from the fracture model on primary osteoblasts. Primary osteoblasts were cultured with medium containing MV-free plasma from fracture model. Data from animals without fracture were set as control. A) Viability of osteoblasts was tested by 3(4,5-Dimethylthiazol-2-yl)-2,5-diphenyltetrazolium bromide (MTT) assay. MV-free plasma from 3 days (group A) post-fracture (0.93 \pm 0.07 vs. $0.55 \pm 0.11$ absorbance (OD $405 \mathrm{nM}), \mathrm{p}=0.0171$ ) significantly improved the viability of osteoblasts. No significance was found within the other groups. B, C, and D) The osteogenesis mRNA expression of runt-related transcription factor 2 (RUNX2), osteocalcin, and collagen 1A were measured by qRT-PCR. The level of osteocalcin mRNA expression after 2 weeks (group C) was significantly higher than that in the control group $(3.48 \pm 2.91$ vs. $1.09 \pm 0.19$ PPIA $\%, p=0.0454)$. There was no significant difference in RUNX2 and collagen $1 \mathrm{~A}$ expression in any of the fracture groups $\left({ }^{*} \mathrm{p}<\right.$ 0.05 ). 


\subsubsection{Viability/proliferation and differentiation of osteoblasts cultured in MV- free plasma culture medium}

To further distinguish the function of MVs and soluble components of the trauma plasma, a MTT assay and mRNA expression of osteogenic genes were also analysed on primary osteoblasts cultured with MV-free plasma from study animals. MV-free plasma from group A (0.93 \pm 0.07 absorbance (OD 405nM), $\mathrm{p}=0.0171)$ ) significantly stimulated viability of primary osteoblasts, as compared with the control group $(0.55$ \pm 0.11 absorbance (OD 405nM)). (Figure 7.9.A) However, no significant differences in viability of primary osteoblasts were found between all the other groups. Osteocalcin expression was significantly higher in group $\mathrm{C}$ as compared with that in the control group (3.48 \pm 2.91 vs. $1.09 \pm 0.19$ PPIA $\%, p=0.0454)$. No differences were detected in RUNX2 and collagen 1A expression (Figure 7.9.B, 7.9.C, and 7.9.D). Comparing MVs in the trauma plasma, MV-free plasma stimulated viability and differentiation of the primary osteoblasts at certain time points.

\subsection{DISCUSSION}

Successful fracture healing involves a complex interaction between a variety of cellular components and signalling molecules ${ }^{1}$. Dysfunction of any of these cellular components or disturbances of signalling mechanisms affects fracture healing. In this study, we aimed to investigate the interaction of systemic MVs from a rat fracture model with osteoblasts. Our main results can be summarized as follows:

1. MVs isolated after a femoral fracture were time-dependently incorporated in osteoblasts and concentrated around the nucleus.

2. The stimulatory effect of trauma plasma on osteoblast proliferation depends on the post-fracture time.

3. MVs from trauma plasma increased the viability of osteoblasts, particularly in the late phase (i.e., 2 weeks post-fracture) after a femoral fracture.

4. Late-phase differentiation of osteoblasts was not stimulated by the MVs in a high extent. In contrast, MV-free plasma seemed to have a stimulatory effect on differentiation in the late phase (2 weeks after fracture).

Previous studies described increased osteoblast growth 2 weeks after a fracture 19,20. In accordance with this finding, in a rat model, we found enhanced growth of osteoblasts stimulated with plasma from 1 and 2 weeks after fracture. The stimulatory effect increased with the time elapsed since trauma. Therefore, plasma, specifically plasma mediators have the potential to modulate fracture healing. Among these mediators, studies reported that TGF $\beta$ and IGF-I represented important growth factors for osteoblasts ${ }^{21}$. TGF- $\beta$ and IGF- 1 were secreted after different inflammatory stimuli and appeared to be good indicators of the fracture-healing process ${ }^{21}$. In our study, both TGF- $\beta 1$ and IGF-1 in MV-free plasma decreased in the first week post fracture. These findings are consistent with those of previous publications ${ }^{21,22}$ and therefore indicate the reliability of our model. To the best of our knowledge, no other studies have described the characteristics and kinetics of TGF- $\beta 1$ and IGF-1 concentrations in MVs after fractures. We found significantly lower levels of TGF- $\beta 1$ and IGF-1 in MVs compared to MV-free plasma, with no relevant changes in intra-vesicular TGF- $\beta 1$ and IGF-1 levels over the entire observation period. 
MVs originate from a variety of cells and indicate the functional status of an organism at a specific time point. In previous studies, MVs were detected in the systemic circulation after diverse insults (e.g. severe trauma, stress) 23-25. Previous studies provided evidence that MVs seemed to be of major importance for intercellular communication in order to regulate bone metabolism 11,12,26,27. In this context, osteoblastic MVs were found to be important for cell-cell communication of osteoblasts with both osteoclasts ${ }^{11}$ and MSCs ${ }^{27}$, these MVs were able to incorporate into cells (e.g., integration of MV-derived osteoclasts into osteoblasts) ${ }^{28-30}$. These findings are in line with those obtained in our study, which revealed the integration of plasma-derived MVs into osteoblasts and their aggregation around the nuclei. Based on the observed accumulation, it can be postulated that MV integration is faster than intracellular degradation of MVs. These results could explain both the potential impact of MVs on cellular function and the importance of MVs as mediators of intracellular communication.

As indicated by the results of the MTT assay in our study, plasma-derived MVs seemed to have the potential to stimulate the viability and proliferation of osteoblasts, particularly in the late phase (i.e., 2 weeks) after fractures, whereas MVindependent mechanisms appeared to be more relevant in the early phase after trauma. Accordingly, others also found stimulatory effects of both bone marrowderived MVs on osteoblast function ${ }^{12}$ and effects of pluripotent stem cell-derived MVs on angiogenesis and osteogenesis ${ }^{26}$.

Previous studies have provided evidence that incorporated extracellular vesicles (e.g., originating from bone marrow stem cells or prostate cancer cell lines) affect osteoblast differentiation ${ }^{12}$. In this context, several studies demonstrated that osteoblast-derived MVs had the potential to stimulate the differentiation of stem cells into osteoblasts ${ }^{31,32}$. In contrast, we found that late-phase differentiation of osteoblasts, represented by osteocalcin synthesis 18 was almost exclusively stimulated by mediators located in plasma but not by plasma-derived MVs alone. These diverse findings might be explained by different factors. First, the low osteocalcin expression after plasma-derived MVs stimulation in our study might be associated with the stimulatory effects of these MVs on the viability and proliferation of osteoblasts. In this context, Owen et al. found low expression of osteocalcin mRNA during the proliferation phase of osteoblasts ${ }^{33}$. Furthermore, studies observed that different factors (e.g., mediators and dynamic cell stretching) that promote the proliferation of osteoblasts simultaneously inhibited osteocalcin expression 34,35. Second, it is possible that the different origins of the investigated MVs (plasma vs. bone marrow stem cells) result in different effects on osteocalcin expression. Therefore, additional studies are needed to investigate the characteristics (e.g., content and receptor status) of MVs originating from diverse cell types.

As determined by the analysis of RUNX 2 and collagen 1A synthesis (early-phase osteoblastic differentiation markers) ${ }^{18}$, we observed no significant differences in the early phase differentiation of osteoblasts among the control and experimental groups following MVs and MV-free plasma stimulation. Several reason might explain this result. First, these findings may point to a comparable effect of both MVs and plasma on early osteoblastic differentiation. Second, as the synthesis of RUNX2 and collagen $1 \mathrm{~A}$ in the trauma groups were not significantly different to that in the controls, the findings may indicate that the primary osteoblasts from this protocol 
are in an early differentiation phase, with a stable expression of RUNX2 and collagen $1 \mathrm{~A}$.

Limitations. First, as our findings were derived from an animal model, they are not directly transferable to humans. However, the results from this study showed a potential role of osteoblast modulation by MVs, which may help in modulating osteogenesis after fracture or orthopaedic operations. Further in vivo and translational studies will be conducted in our lab in the future. Second, the amount of plasma harvested from this rat trauma model was limited, whereas the consumption of the cell culture and tests was high. This limited the possibility of further analyses, such as MV phenotype, miRNA analysis and in vivo studies. Thus, additional studies are needed to further elucidate the role of MVs in osteoblastic function post-fracture. In the following studies, larger animals may be needed in studies to increase both the availability of material for analysis, as well as the translational relevance. Finally, the centrifuge protocol for MV isolation does not exclude the presence of small amounts of other components (e.g., exosomes) that might have an impact on osteoblast functions ${ }^{14}$.

\subsection{CONCLUSIONS}

The fracture healing process involves a complex network of signal transduction between a variety of cells. Our study showed a potential effect of MVs on regulating fracture healing, by modulating the viability and proliferation of osteoblasts. Therefore, MVs may possibly have potential therapeutic uses in cases of fracture healing disturbances. This should be clarified in further translational studies.

\subsection{ACKNOWLEDGEMENTS}

This work was supported by the Confocal Microscopy Facility of the Interdisciplinary Centre for Clinical Research (IZKF) Aachen.

Source of Funding. This research project was supported by the START-Program of the Faculty of Medicine, RWTH Aachen University, Germany.

Conflict of Interest Statement. Each author certifies that he or she has no commercial associations (e.g. consultancies, stock ownership, equity interest, patent/licensing arrangements, etc.) that might pose a conflict of interest in connection with the submitted article.

\subsection{REFERENCES}

1. Qiao Z, Greven J, Horst K, et al. Fracture Healing and the Underexposed Role of Extracellular VesicleBased Cross Talk. Shock 2018; 49(5): 486-96.

2. Cocucci E, Racchetti G, Meldolesi J. Shedding microvesicles: artefacts no more. Trends in cell biology 2009; 19(2): 43-51.

3. Dignat-George F, Boulanger CM. The many faces of endothelial microparticles. Arteriosclerosis, thrombosis, and vascular biology 2011; 31(1): 27-33.

4. Lawson C, Vicencio JM, Yellon DM, Davidson SM Microvesicles and exosomes: new players in metabolic and cardiovascular disease. The Journal of endocrinology 2016; 228(2): R57-71.

5. Losche W, Scholz T, Temmler U, Oberle V, Claus RA. Platelet-derived microvesicles transfer tissue factor to monocytes but not to neutrophils. Platelets 2004; 15(2): 109-15.

6. Laffont B, Corduan A, Rousseau M, et al. Platelet microparticles reprogram macrophage gene expression and function. Thrombosis and haemostasis 2016; 115(2): 311-23.

7. Laffont B, Corduan $\mathrm{A}, \mathrm{Ple} \mathrm{H}$, et al. Activated platelets can deliver mRNA regulatory Ago 2*microRNA complexes to endothelial cells via microparticles. Blood 2013; 122(2): 253-61.

8. Dang PN, Dwivedi N, Phillips LM, et al. Controlled Dual Growth Factor Delivery From Microparticles Incorporated Within Human Bone Marrow-Derived Mesenchymal Stem Cell Aggregates for Enhanced Bone Tissue Engineering via Endochondral 
Ossification. Stem cells translational medicine 2016; 5(2): 206-17.

9. Zhang J, Ren J, Chen H, Geng Q. Inflammation induced-endothelial cells release angiogenesis associated-microRNAs into circulation by microparticles. Chinese medical journal 2014; 127(12): 2212-7.

10. Deregibus MC, Cantaluppi V, Calogero R, et al. Endothelial progenitor cell derived microvesicles activate an angiogenic program in endothelial cells by a horizontal transfer of mRNA. Blood 2007; 110(7): 2440-8.

11. Deng L, Wang Y, Peng Y, et al. Osteoblast-derived microvesicles: A novel mechanism for communication between osteoblasts and osteoclasts. Bone 2015; 79: 37-42.

12. Qin Y, Wang L, Gao Z, Chen G, Zhang C. Bone marrow stromal/stem cell-derived extracellular vesicles regulate osteoblast activity and differentiation in vitro and promote bone regeneration in vivo. Scientific reports 2016; 6: 21961.

13. Wang SJ, Lewallen DG, Bolander ME, Chao EY, Ilstrup DM, Greenleaf JF. Low intensity ultrasound treatment increases strength in a rat femoral fracture model. Journal of orthopaedic research : official publication of the Orthopaedic Research Society 1994; 12(1): 40-7.

14. Dey-Hazra E, Hertel B, Kirsch T, et al. Detection of circulating microparticles by flow cytometry: influence of centrifugation, filtration of buffer, and freezing. Vascular health and risk management 2010; 6: 1125-33.

15. Orriss IR, Taylor SE, Arnett TR. Rat osteoblast cultures. Methods in molecular biology 2012; 816: 3141.

16. Choi H, Srikanth S, Atti E, et al. Deletion of Orai1 leads to bone loss aggravated with aging and impairs function of osteoblast lineage cells. Bone reports 2018; 8: $147-55$.

17. Livak KJ, Schmittgen TD. Analysis of relative gene expression data using real-time quantitative PCR and the 2(-Delta Delta C(T)) Method. Methods 2001; 25(4): 402-8.

18. Tang $Q$, Tong $M$, Zheng G, Shen L, Shang P, Liu H. Masquelet's induced membrane promotes the osteogenic differentiation of bone marrow mesenchymal stem cells by activating the Smad and MAPK pathways. American journal of translational research 2018; 10(4): 1211-9.

19. Iwaki A, Jingushi S, Oda Y, et al. Localization and quantification of proliferating cells during rat fracture repair: detection of proliferating cell nuclear antigen by immunohistochemistry. Journal of bone and mineral research : the official journal of the American Society for Bone and Mineral Research 1997; 12(1): 96-102.

20. Wildemann B, Schmidmaier G, Ordel S, Stange R, Haas NP, Raschke M. Cell proliferation and differentiation during fracture healing are influenced by locally applied IGF-I and TGF-beta1: comparison of two proliferation markers, PCNA and BrdU. Journal of biomedical materials research Part B, Applied biomaterials 2003; 65(1): 150-6.

21. Fischer C, Doll J, Tanner M, et al. Quantification of TGF-ss1, PDGF and IGF-1 cytokine expression after fracture treatment vs. non-union therapy via masquelet. Injury 2016; 47(2): 342-9.

22. Chen QQ, Wang WM. Expression of FGF-2 and IGF1 in diabetic rats with fracture. Asian Pacific journal of tropical medicine 2014; 7(1): 71-5.

23. Matijevic N, Wang YW, Holcomb JB, Kozar R, Cardenas JC, Wade CE. Microvesicle phenotypes are associated with transfusion requirements and mortality in subjects with severe injuries. Journal of extracellular vesicles 2015; 4: 29338.

24. Curry N, Raja A, Beavis J, Stanworth S, Harrison P. Levels of procoagulant microvesicles are elevated after traumatic injury and platelet microvesicles are negatively correlated with mortality. Journal of extracellular vesicles 2014; 3: 25625.

25. Fleshner M, Crane CR. Exosomes, DAMPs and miRNA: Features of Stress Physiology and Immune Homeostasis. Trends in immunology 2017; 38(10): 768-76.

26. Qi X, Zhang J, Yuan H, et al. Exosomes Secreted by Human-Induced Pluripotent Stem Cell-Derived Mesenchymal Stem Cells Repair Critical-Sized Bone Defects through Enhanced Angiogenesis and Osteogenesis in Osteoporotic Rats. International journal of biological sciences 2016; 12(7): 836-49.

27. Xu JF, Yang GH, Pan XH, et al. Altered microRNA expression profile in exosomes during osteogenic differentiation of human bone marrow-derived mesenchymal stem cells. PloS one 2014; 9(12): e114627.

28. Sun W, Zhao C, Li Y, et al. Osteoclast-derived microRNA-containing exosomes selectively inhibit osteoblast activity. Cell discovery 2016; 2: 16015.

29. Li D, Liu J, Guo B, et al. Osteoclast-derived exosomal miR-214-3p inhibits osteoblastic bone formation. Nature communications 2016; 7: 10872.

30. Ye Y, Li SL, Ma YY, et al. Exosomal miR-141-3p regulates osteoblast activity to promote the osteoblastic metastasis of prostate cancer. Oncotarget 2017; 8(55): 94834-49.

31. Nair R, Santos L, Awasthi S, et al. Extracellular vesicles derived from preosteoblasts influence embryonic stem cell differentiation. Stem cells and development 2014; 23(14): 1625-35.

32. Cui Y, Luan J, Li H, Zhou X, Han J. Exosomes derived from mineralizing osteoblasts promote ST2 cell osteogenic differentiation by alteration of microRNA expression. FEBS letters 2016; 590(1): 18592.

33. Owen TA, Aronow M, Shalhoub V, et al. Progressive development of the rat osteoblast phenotype in vitro: reciprocal relationships in expression of genes associated with osteoblast proliferation and differentiation during formation of the bone extracellular matrix. Journal of cellular physiology 1990; 143(3): 420-30.

34. Viereck V, Siggelkow H, Tauber S, Raddatz D, Schutze N, Hufner M. Differential regulation of Cbfa1/Runx2 and osteocalcin gene expression by vitamin-D3, dexamethasone, and local growth factors in primary human osteoblasts. Journal of cellular biochemistry 2002; 86(2): 348-56. 
35. Kaspar D, Seidl W, Neidlinger-Wilke C, Ignatius A, Claes L. Dynamic cell stretching increases human osteoblast proliferation and CICP synthesis but decreases osteocalcin synthesis and alkaline phosphatase activity. Journal of biomechanics 2000; 33(1): 45-51. 


\title{
Altered cell surface receptor dynamics and circulatory occurrence of neutrophils in a small animal fracture model.
}

\section{Pathology - Research and Practice}

2020 Jul 12;216(10):153108. doi: 10.1016/j.prp.2020.153108 [Online ahead of print] PMID: 32853946

\author{
Michel P.J. Teuben ${ }^{1,2, *}$ \\ Martijn Hofman ${ }^{3, *}$ \\ Johannes Greven ${ }^{3}$ \\ Qiao Zhi ${ }^{3}$ \\ Alba Shehu ${ }^{1,2}$ \\ Henrik Teuber ${ }^{1,2}$ \\ Frank Hildebrand ${ }^{3}$ \\ Roman Pfeifer ${ }^{1,2}$ \\ Hans-Christoph Pape ${ }^{1,2, \dagger}$ \\ Frank Hildebrand ${ }^{3, \dagger}$
}

1 Department of Traumatology, University Hospital Zürich, Switzerland

2 Harald Tscherne Research Laboratory, University Hospital Zürich, Switzerland

${ }^{3}$ Department of Orthopaedic Trauma and Reconstructive Surgery, University Medical Center RWTH Aachen, Germany

* Authors contributed equally

+ Authors contributed mutually 


\section{ABSTRACT}

Introduction: Excessive activation of the immune response after femoral fractures and fracture fixation is potentially associated with the development of systemic and local complications, particularly in multiple trauma patients. A dysregulated function of neutrophils, the most prevailing immune cells in circulation, has been discussed as a central pathophysiological background for these unfavourable post-traumatic courses. Our aim was to investigate alterations in activity and functionality as expressed by the cell surface receptor dynamics of circulatory neutrophils after femoral fracture and intramedullary stabilization.

Material and Methods: After intramedullary stabilization, an isolated femur fracture was induced in 18 Sprague-Dawley rats. Animals were terminated at different time points, i.e. after $3(n=5$, group $3 d), 7(n=5$, group $7 d)$ and $14(n=5$, Group $14 d)$ days and grouped accordingly. Additionally, baseline measurements were performed in one control animal per study group $(n=3)$ after anaesthesia induction and termination, without prior intramedullary nailing and fracture induction. The numbers and cell surface expression of CD11b, CD11a, CD62L, and CD49d of circulating neutrophils were compared between groups.

Results: Neutrophil numbers were significantly reduced at 3 days compared with baseline measurements $\left(1.2 \times 10^{5}\right.$ vs. $6.3 \times 10^{5}$ cells $\left./ \mathrm{ml}, \mathrm{p}<0.01\right)$. By day 7 , neutrophil counts significantly increased back to homeostatic levels $(p<0.05)$. At day 3, CD11bexpression was significantly reduced, whereas CD11a-expression was increased compared with the baseline measurements $(\mathrm{p}<0.05)$. At day 7 , the circulatory

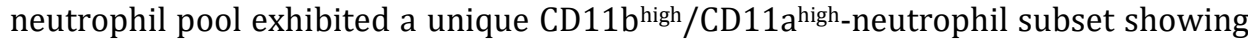
a significantly increased co-expression of CD49d. The expression of CD62L did not change significantly throughout the experiment compared with baseline measurements.

Conclusions: This descriptive small animal fracture study is the first to show that an intramedullary stabilized femur fracture is associated with a temporary reduction in circulatory neutrophil count and concurrent changes in circulatory neutrophil function. Moreover, we demonstrated that the restoration to homeostatic neutrophil activation status occurs concomitantly with the appearance of a novel neutrophil subtype (CD11 $\left.\mathrm{b}^{\text {high }} / \mathrm{CD} 11 \mathrm{a}^{\text {high }}\right)$ in circulation.

Our fundamental new findings of the changes in circulatory neutrophil count and functionality after trauma form an excellent basis for future studies to further elucidate the role of neutrophils as activators and regulators of different posttraumatic processes, potentially resulting in local (e.g., fracture healing disturbances) or systemic (e.g., MODS) complications. This might result in the development of specific therapies to reduce adverse outcomes after trauma. 


\subsection{INTRODUCTION}

It is well-known that particularly in subgroups of multiple trauma patients, femur fractures and their intramedullary stabilization might result in a higher incidence of post-traumatic complications ${ }^{1,2}$. Post-traumatic activation of the immune response has been shown to play a central role in these unfavourable events over the clinical course. It is assumed that neutrophils, the most prevailing immune cells in the human circulation system, play a central role in pathophysiological processes, potentially leading to systemic and local complications ${ }^{3,4}$.

At the local level at the fracture site, neutrophils are one of the first cells that permeate into the fracture hematoma. Here, they differentiate into different subsets, synthesize an "emergency extra cellular matrix," and initiate the inflammation stage of fracture healing ${ }^{2,5}$. At this stage, recruited immune cells clear the fracture area from pathogens and cell debris, restricting tissue damage ${ }^{6}$. On the other hand, if this stage is not terminated adequately, damage of even uninjured tissue because of an excessive stimulation of neutrophils can occur ${ }^{6}$. In this way, even systemic complications can occur because of the infiltration of neutrophils into lung parenchyma, with the subsequent formation of oedema, leading to tissue and, eventually, organ damage ${ }^{7-9}$.

Neutrophil tissue homing is regulated by alterations in the expression of cell surface receptors. Various selectins, including L-selectin (CD62L), are initially upregulated and later shed from the cell surface to enable vessel wall adherence and to initiate deceleration and rolling. In addition, integrin upregulation, including macrophage-1 antigen (Mac-1 or CD11b) and lymphocyte function-associated antigen-1 (LFA-1 or CD11a), achieves actual neutrophil adhesion and extravasation ${ }^{10}$. However, in vitro studies suggest that there is a functional overlap and that integrins are not only involved in adhesion and transmigration, but also in rolling ${ }^{11}$.

The aforementioned changes of the local release and/or differentiation of diverse neutrophil subsets have also been described after fractures ${ }^{5}$. These subsets present with various surface receptor expressions, which, in turn, result in different neutrophil characteristics. However, knowledge of the specific influence of an isolated stabilized femoral fracture on the systemic neutrophil pool, especially throughout the later post-traumatic phases, is sparse. Indeed, the alterations in circulatory neutrophil number and their cell surface expression of selectins and integrins following fracture and intramedullary nailing have never been investigated.

Knowledge of the time course and specific pattern of neutrophil surface receptor expression after trauma is of the utmost importance for the future development of therapeutic options to modulate the role and homing of neutrophils in injured tissue. Therefore, we investigated if femoral fractures and intramedullary nailing are associated with alterations in circulatory neutrophil counts and circulatory neutrophil surface receptor expression of selectins and integrins. 


\subsection{MATERIALS AND METHODS}

\subsubsection{Housing}

Our study cohort consisted of 21 adult female Sprague-Dawley rats weighing approximately $250 \mathrm{~g}$; the rats were obtained from Envigo B.V. (Horst, Netherlands).

All the animals were specifically pathogen free according to the Federation of European Laboratory Animal Science Associations' recommendations. The animals were housed and the experiments performed at the Institute of Laboratory Animal Science, University of Aachen Medical Center, Germany, with the approval of the Governmental Animal Care and Use Committee (Landesamt für Natur, Umwelt und Verbraucherschutz, North Rhine-Westphalia, Recklinghausen, Germany; Protocol No. 84-02.04.2015.A078). The animals were housed under controlled temperature $\left(20 \pm 2{ }^{\circ} \mathrm{C}\right)$ and air humidity (45-65\%), with a 12 hour lightdark cycle and a light intensity of $<200$ lux. Food and water were offered ad libitum. Prior to study inclusion, all animals were kept in groups for 1 week in the laboratory premises to allow for acclimatization. Throughout the entire experiment, the rats underwent physical examinations according to a "score sheet" documentation ${ }^{12}$ and the "body condition scoring" according to Hickman ${ }^{13}$ to obtain the general health status.

In order to optimize standardization of this long-term observation study, female animals were utilized, as variations in animal weight over time are smaller than observed in their male counterparts. Because of the protective effects of female hormones in cases of inflammatory stimuli, all animals were confirmed to be in the same "metestrus" phase of the menstrual cycle because this phase is characterized by low oestrogen and progesterone levels 14,15. The menstrual cycle phase was identified by the assessment of vaginal swabs according to Marcondes et al. ${ }^{16}$.

\subsubsection{Experimental design and study groups}

In order to minimize usage of experimental animals in the current study, a baseline measurement was performed after anaesthesia and termination after 3 days $(n=1)$, 7 days $(n=1)$, and 14 days $(n=1)$, without prior intramedullary nailing and fracture induction. Data of control animals was pooled and analysed as Group control.

In all other animals $(n=18)$, after anaesthesia, an intramedullary Kirschner wire was inserted, and a standardized femoral fracture was induced at the right side. The animals were then randomly divided into three study groups according to the observation period:

Group 3d : 3 days of observation after fracture induction

Group 7d : 7 days of observation after fracture induction

Group 14d : 14 days of observation after fracture induction

The reasons for excluding animals were as follows: death from anaesthetic complications, open fracture, comminuted fracture, implant failure, wound dehiscence, or infection.

\subsubsection{Anaesthesia and pain management}

The animals received buprenorphine hydrochloride $(0.03-0.05 \mathrm{mg} / \mathrm{kg}$ s.c.) as a multimodal analgesic at 30 minutes before operation. The operative procedures were performed under general anaesthesia induced with ketamine (100 mg/kg i.p.) and xylazine $(2 \% ; 10 \mathrm{mg} / \mathrm{kg}$ i.p.) and, if necessary, extended with isoflurane 
inhalation (2.0-2.5 Vol.\%). The toe pinch reflex was used to ensure adequate anaesthesia. Postoperative analgesia was ensured with buprenorphine hydrochloride (0.03-0.05 mg/kg s.c.) every 6 hours for the first 24-48 hours. Subsequently, it was given twice daily during the entire period of the experiment. All animals were allowed to mobilize freely directly after the operative procedure. The animals were evaluated three times per day using the score sheet evaluation ${ }^{12}$. In the potential case of clinical signs of uncompensated/enhanced pain, animals are to be withdrawn from the study. Similar painkilling protocols were applied to control animals during the observation period.

\subsubsection{Standardized femoral fracture}

Sterilized implants (autoclave processing) and surgical equipment was used. Furthermore, procedures were performed using sterile gowns, gloves, surgical mask and theatre caps. After anaesthesia induction, the animals were placed on a heated pad $\left(37^{\circ} \mathrm{C}\right)$, and their eyes were covered with a moistening ointment. Their right hind leg was shaved, disinfected, and draped. Then, a para-patellar incision was made, the patella was everted laterally, and a $1 \mathrm{~mm}$ stainless-steel intramedullary Kirschner wire (Königsee Implantate $\mathrm{GmbH}$, Allendorf, Germany) was inserted in a retrograde manner. Its placement was confirmed by fluoroscopy. The sharp end of the Kirschner wire was placed to the proximal end of the femur. The Kirschner wire was cut flush with the intercondylar notch, and the proximal end was bent over the greater trochanter, cut, and hidden subcutaneously. The distal end of the Kirschner wire was than shortened underneath the cartilaginous surface. The patella was repositioned, and the wounds were sutured (Ethicon Inc., Somerville, NJ, USA) in layers. Surgical procedures have been described in detail by Klueter et al. ${ }^{17}$. A transverse fracture was induced with a blunt guillotine according to the method by Bonnarens and Einhorn 18. A fluoroscopic evaluation of the fractured side was performed directly after fracture induction. An example of fracture fixation adequacy and radiographic imaging is provided in figure 8.1.
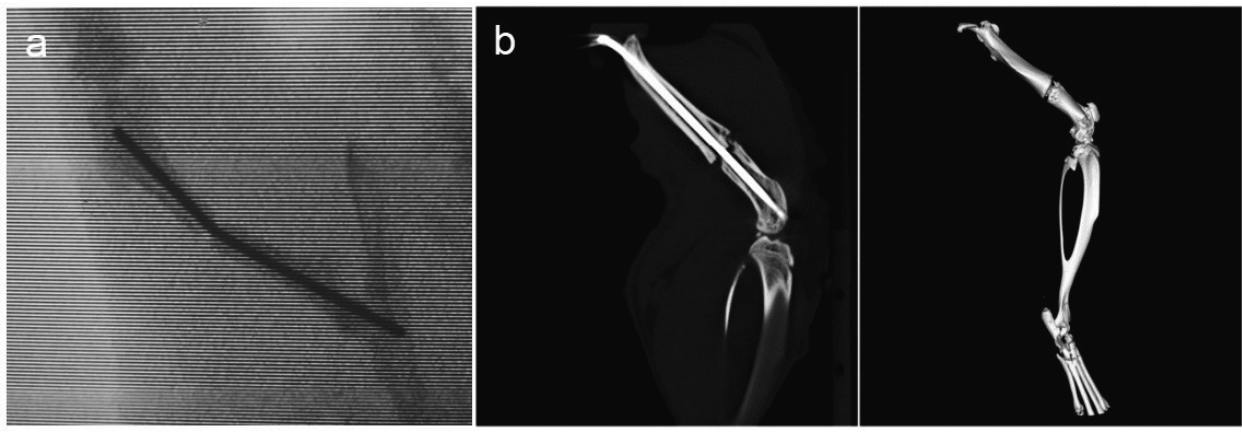

Figure 8.1. Fracture confirmation by X-ray and post-mortem micro-CT imaging. (a) X-ray confirmation of a non-displaced midshaft femur fracture and adequate nail positioning. (b) Post-mortem micro-CT analysis of the fractured femur to evaluate fracture healing, rotation and displacement of the fracture or the nail. 


\subsubsection{Blood collection and euthanasia}

In accordance with animal grouping, animals were euthanized at three different time points $(3 \mathrm{~d}, 7 \mathrm{~d}$, and $14 \mathrm{~d})$ by cardiac puncture with an ethylenediaminetetraacetic acid (EDTA)-coated syringe. Prior to cardiac puncture, animals were put under general anaesthesia induced with ketamine (100 mg/kg i.p.) and xylazine $(2 \% ; 10 \mathrm{mg} / \mathrm{kg}$ i.p.). After cardiac puncture, an overdose of Isoflurane was provided and manual cervical dislocation was applied.

\subsubsection{Sampling}

Blood samples were lysed twice in an ice-cold isotonic $\mathrm{NH}_{4} \mathrm{Cl}$ solution. Cells were then washed twice in a FACS (fluorescence-activated cell sorting) buffer solution (phosphate buffered saline supplemented with $0.5 \%$ bovine serum albumin and 0.5 mM EDTA). Cell suspensions were then incubated with conjugated mouse anti-rat monoclonal antibodies. The following commercially available mouse anti-rat monoclonal antibodies were utilized: CD62L clone OX-85 (AbD Serotec, Düsseldorf, Germany) / fluorescein isothiocyanate (FITC), CD11b clone M1/70 (eBioscience Vienna, Austria) / R-phycoerythrin (RPE), CD49d (VLA-4) clone MRalpha4-1 (Becton \& Dickinson, Mountain View, CA, USA) / peridinin-chlorophyll-protein-cyanine 5.5 (PerCP-Cy5.5), CD11a clone WT.1 (AbD Serotec, Düsseldorf, Germany) / allophycocyanin $(A P C)$, and Granulocytes mouse anti-rat clone RP-1 (Becton \& Dickinson, Mountain View, CA, USA) / allophycocyanin-cyanine 7 (APC-Cy7). Details on flow analyses are provided in figure 8.2.

\subsubsection{Neutrophil cell surface marker expression}

Neutrophil cell membrane receptor expression levels of CD62L (L-selectin), CD11b (macrophage-1 antigen (Mac-1)), and CD11a (lymphocyte function-associated antigen-1 (LFA-1)) were measured by flow cytometry with a Canto II-device (Becton \& Dickinson, Mountain View, CA, USA) and FACS Diva software (Becton \& Dickinson, Mountain View, CA, USA). Neutrophils were identified using a four-step gating strategy (Figure 8.2.). In steps one and two, debris and doublets were excluded. In step three, cells with a neutrophil specific forward scatter/side scatter-pattern were included. Finally, RP-1 positive cells were included. The utilized gating strategy has previously been validated for analysis of both blood and tissue neutrophils 19-22. From each sample, a minimum of 200,000 cells were measured. Cell viability was confirmed in separate pilot experiments. Because cells were stained and measured within 2 hours after blood collection, maximal neutrophil-viability was ensured, and cell fixation was not indicated. The dynamics of cell surface receptors was compared between the groups. Additionally, the current study aimed to identify novel subsets that play a role in the inflammatory response to trauma.

\subsubsection{Neutrophil count}

The total cell count of viable neutrophils was determined using a Neubauer improved grid haemocytometer (LW Scientific, Lawrenceville, USA). This device is optimized for leukocyte versus non-leukocyte differentiation of cell suspensions. Two independent samples were taken from lysed blood samples. Cell suspensions were stained with Tryptan blue (Thermo Fisher Scientific, Waltham, MA, USA) and then separately filled into two counting areas on the counting chambers. The total cell counts of large, viable cells were documented, and an average value was calculated. 
Thereafter, total neutrophil counts were determined by the ratio of RP-1 (a specific neutrophil marker in rats) positive cells: total gated white blood cells (cells) as measured by flow cytometry. This method has been described and validated by Krajnar et al. ${ }^{21}$.
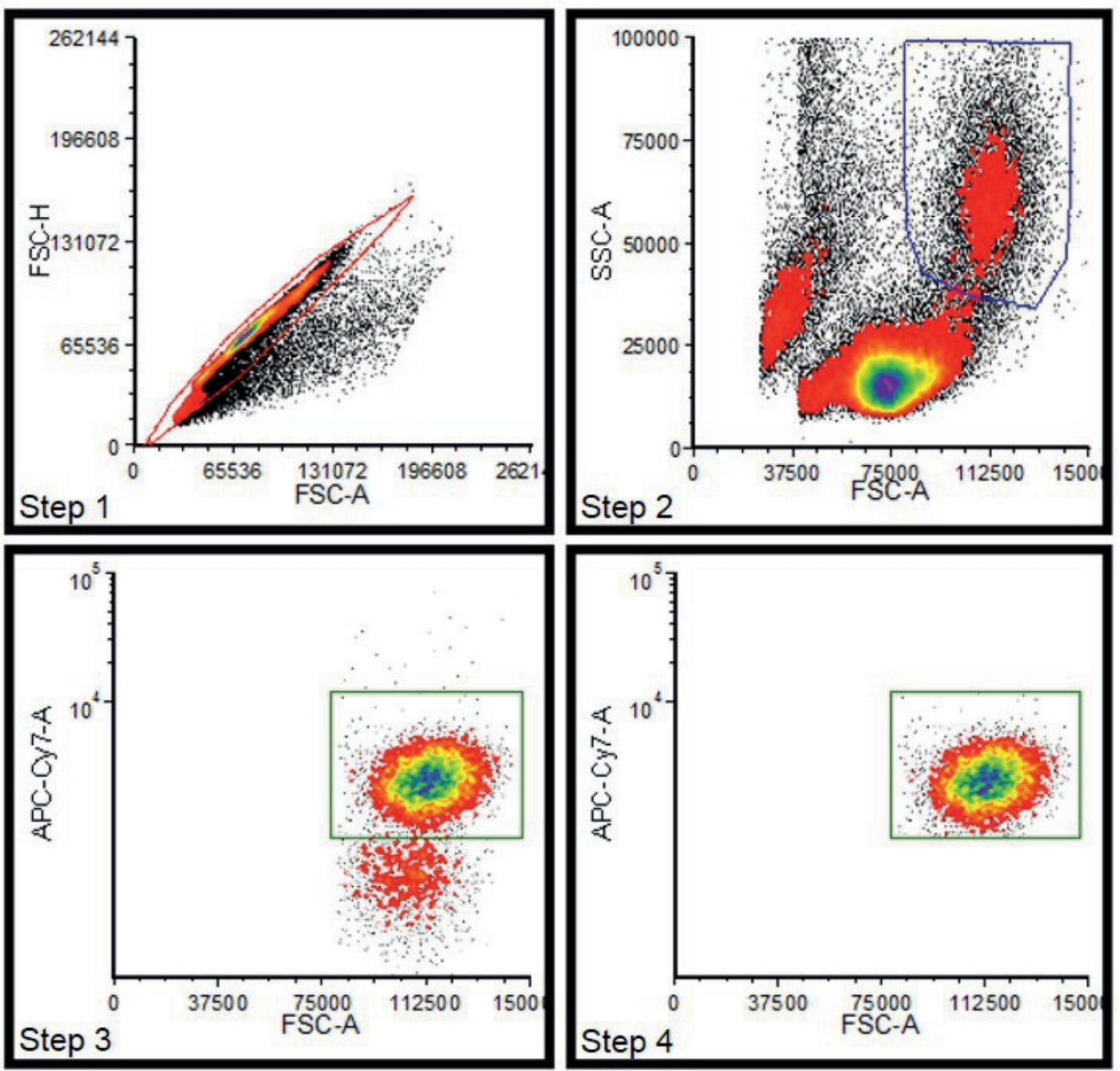

Figure 8.2. 4-step neutrophil identification and gating strategy in peripheral murine blood samples. Neutrophils were identified through a 4-step gating strategy including $(1,2)$ exclusion of debris and doublets, (3) inclusion of cells with a neutrophil specific FSC/SSC-pattern, and (4) inclusion of RP-1 positive cells. Cell viability was confirmed in separate pilot experiments. As cells were stained and measured within 2 hours after blood sampling there was maximal PMN-viability and no need for cell fixation.

\subsubsection{Endpoint and power analysis}

The primary endpoint of our study was an alteration in circulatory neutrophil numbers in the early fracture healing period of 14 days. A secondary endpoint was an alteration in neutrophil activity, as measured by neutrophil cell surface 
expression of CD11a, CD11b, and CD62L. Our sample size calculation was based on alterations in circulatory neutrophil numbers over time in a rat study from McManus 56, which demonstrates the raw data of circulatory neutrophil numbers from the control (1565 \pm 387 cells $/ \mathrm{mm}^{3}$ ) and intervention groups (3999 \pm 787 cells $/ \mathrm{mm}^{3}$ ) 9 days after insult. According to our power analysis, groups with at least four animals will provide $90 \%$ power at an $\alpha$-level of 0.05 . To compensate for higher variability in our model and potential mortality, we decided to include six animals in each intervention group.

\subsubsection{Data analysis}

Statistical analysis was performed using SPSS (version 20.0; IBM Inc., Somers, NY, USA) and GraphPad Prism (version 7; San Diego, USA). All data are presented as the mean and standard deviation unless described otherwise. Comparison of the data with control values and between groups was performed using Mann-Whitney's $U$ test. In general, a two-sided $p$-value $<0.05$ was considered significant.

\subsection{RESULTS}

All animals survived the observation period, and no animals had to be excluded according to our exclusion criteria.

\subsubsection{Circulatory neutrophil count}

The absolute neutrophil count significantly decreased in Group 3d compared with the baseline conditions $\left(1.2 \times 10^{5}\right.$ vs. $6.3 \times 10^{5}$ cells / ml, p < 0.01). However, circulatory neutrophil numbers returned to homeostatic levels after 7 days of observation $(\mathrm{p}<0.05)$ and remained stable at day $14(\mathrm{p}<0.05)$ (Figure 8.3.).

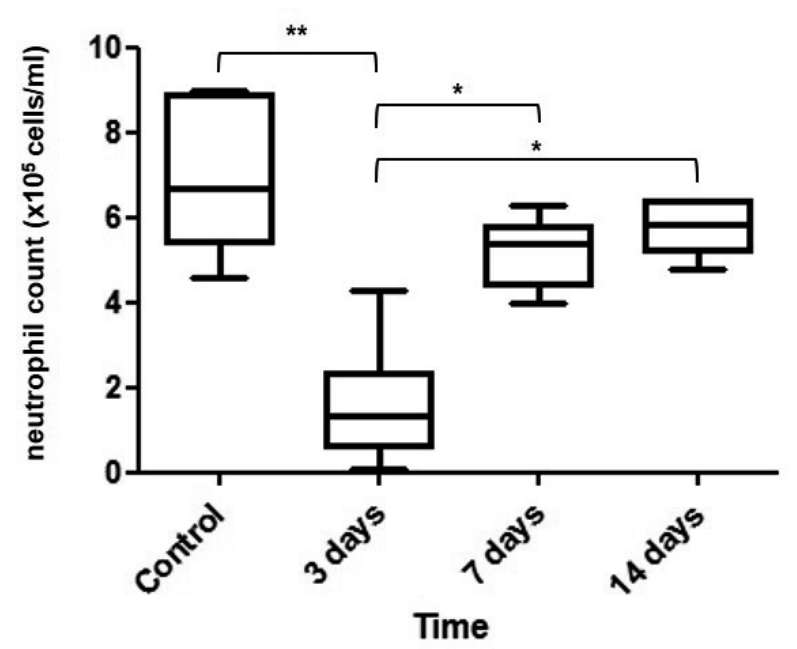

Figure 8.3. Circulatory neutrophil count in early fracture healing. A significant difference was seen between the baseline measurement (control) and animals terminated 3 days after surgery $(* * p<0.01)$. Furthermore, a significant rise in neutrophil count was observed between day 3 and day $7\left(^{*} \mathrm{p}<0.05\right)$. No differences were found between the baseline measurement (control) and counts at day 7 and day 14. 


\subsubsection{Neutrophil cell surface expression of L-selectin (CD62L)}

The expression of L-selectin (CD62L) at the surface of circulatory neutrophils decreased significantly in Group 7d and Group 14d compared with Group 3d, but no significant difference occurred between the baseline measurement and all three fracture groups (Figure 8.4.a).
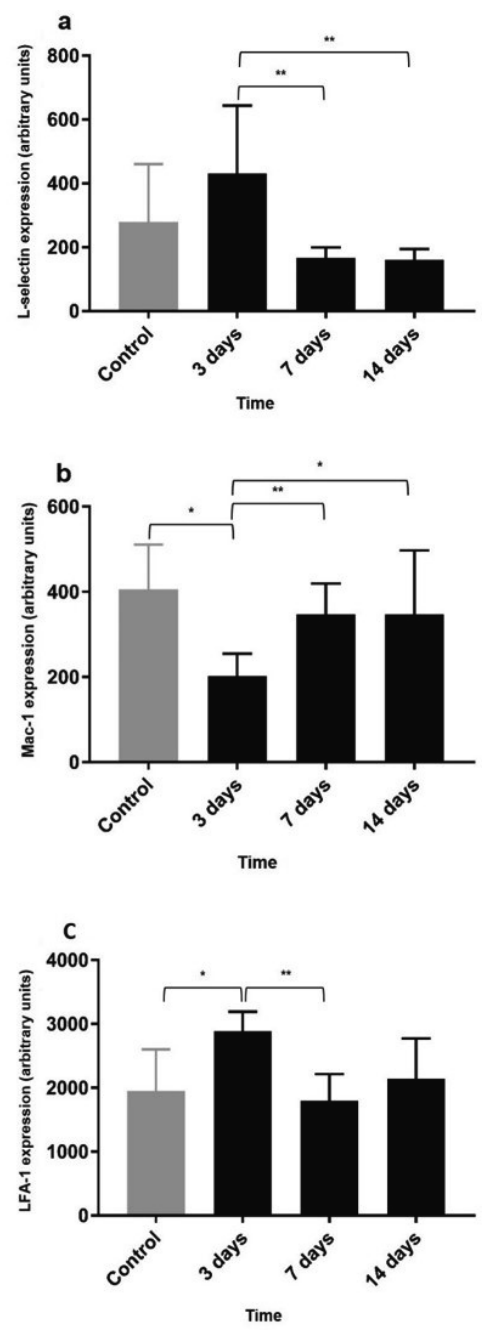

Figure 8.4. Cell surface expression of selectins/integrins in circulatory neutrophils. (a) L-selectin (CD62L) expression in circulatory neutrophils. Significant differences were seen between day 3 and day 7 , as well as day 14 , after intramedullary nailing and fracture induction $(* * p<0.01$ ). No significant differences were found between the intervention groups and the baseline measurement. (b) Mac-1 (CD11b) expression in circulatory neutrophils. A significant decrease of Mac-1 expression was seen at day 3 compared with the baseline measurement $(* \mathrm{p}<0.05)$. After 7 days, the expression returned to baseline values and remained at this level throughout day 14. (c) LFA-1 (CD11a) expression in circulatory neutrophils. Expression in mean fluorescence intensity (MFI) in arbitrary units (AU) over time. Statistical significance: $* \mathrm{p}<0.05 ; * * \mathrm{p}<0.01$. 


\section{CHAPTER 8 |}

\subsubsection{Neutrophil cell surface expression of Mac-1 (CD11b)}

The expression of Mac-1 (CD11b) at the surface of circulatory neutrophils was significantly decreased in Group 3d compared with the baseline measurement ( $\mathrm{p}<$ 0.05). In Group 7d and Group 14d, the expression of Mac-1 (CD11b) returned to baseline values again. These values were significantly increased compared with the values at day 3 (day 7: $\mathrm{p}<0.01$, day 14: $\mathrm{p}<0.05$ ) (Figure 8.4.b).

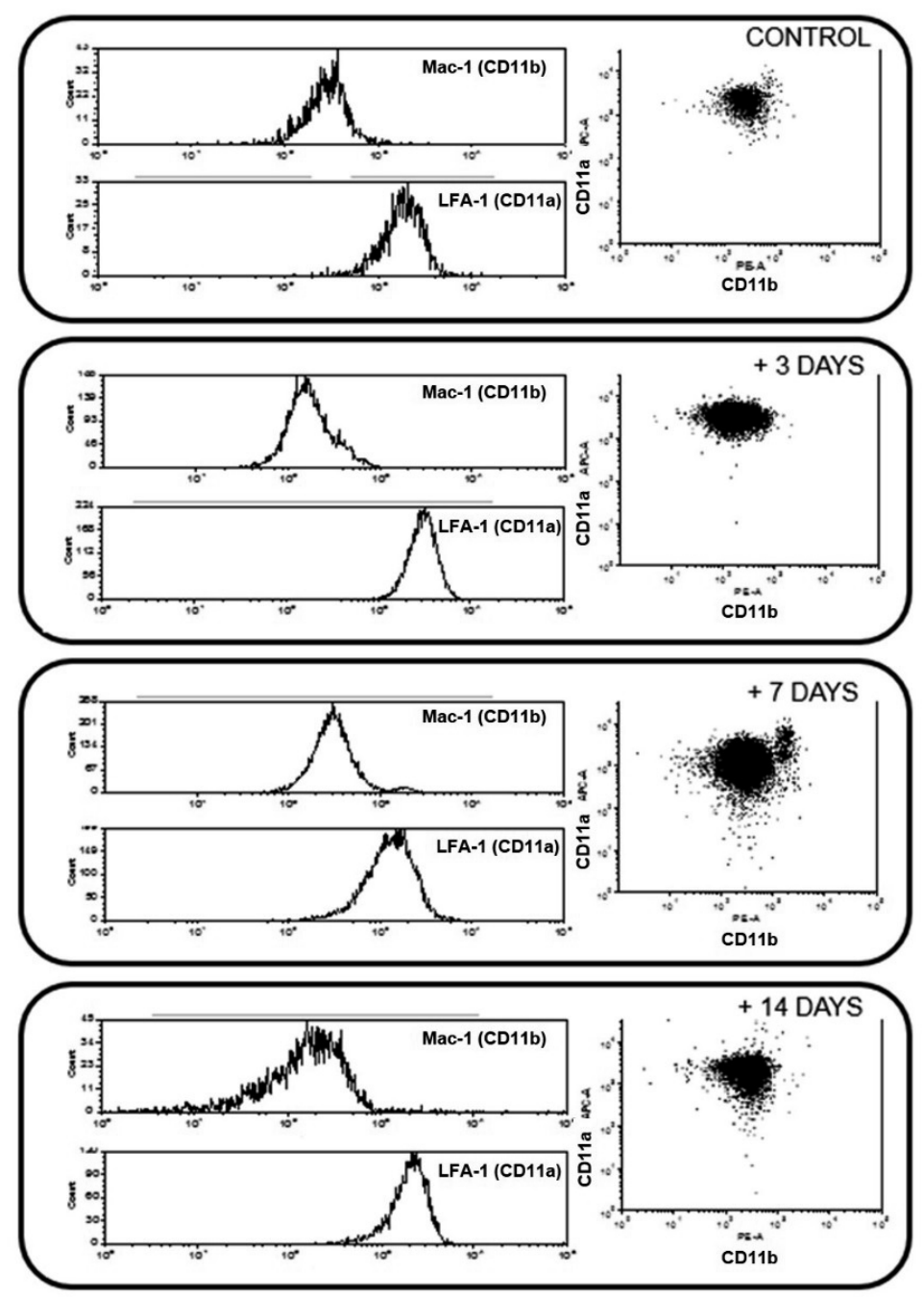

Figure 8.5. Flow cytometric analysis of changes in circulatory neutrophil integrin cell surface expression. Representative examples of alterations in LFA-1 (CD11a) and Mac-1 (CD11b) expression during fracture healing were selected. Histograms (left): These show the cell surface expression levels of either Mac-1 (CD11b) or LFA-1 (CD11a) on the x-axis and cell count on the y-axis. Dot plot (right): A twodimensional analysis was added to highlight the relationship between circulatory neutrophil Mac-1 (CD11b) and LFA-1 (CD11a) expression. Here, Mac-1 (CD11b)-PE expression in mean fluorescence intensity (MFI) in arbitrary units (AU) is shown on the x-axis, and LFA-1 (CD11a)-APC mean fluorescence levels $(\mathrm{AU})$ are plotted on the y-axis. 


\subsubsection{Neutrophil cell surface expression of LFA-1 (CD11a)}

The expression of LFA-1 (CD11a) at the surface of circulatory neutrophils significantly increased temporarily in Group 3d when compared with the baseline measurement ( $\mathrm{p}<0.05)$ and with Group 7d ( $<<0.01)$. LFA-1 (CD11a) expression levels returned to homeostatic baseline levels by day 7 and remained at baseline until the end of the experiment (Figure 8.4.c).

Fractions of neutrophil subtypes

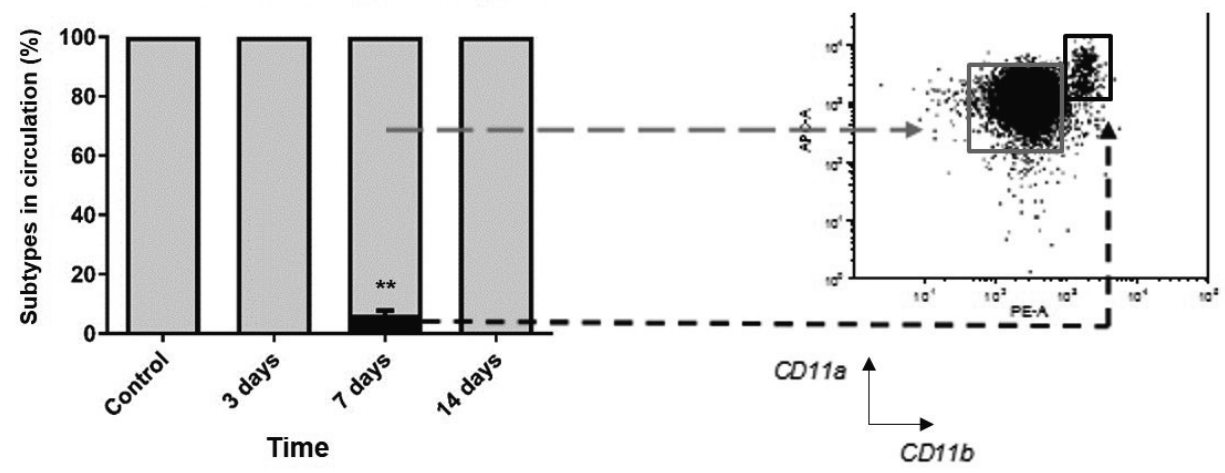

Figure 8.6. Fractions of neutrophil subtypes. Bar chart (left): The grey bars in the bar chart represent the percentage of regular non-CD11 $\mathrm{b}^{\text {high }} / \mathrm{CD} 11 \mathrm{a}^{\text {high }}$ neutrophil subtypes, and the black bar represents the percentage (7.62\%, range: $5.56-9.24 \%)$ of novel CD11 bigh $/$ DD11ahigh cells in peripheral blood. The percentage of CD11 $\mathrm{b}^{\text {high }} / \mathrm{CD} 11 \mathrm{a}^{\text {high }}$ neutrophils was significantly higher on postoperative day 7 compared with all other groups. Dot plot (right): A duplicate of a two-dimensional dot plot from a representative example provides an example of the utilized gating strategy. Statistical significance: ${ }^{* *} \mathrm{p}<0.01$.

\subsubsection{Co-expression patterns and the interplay between circulatory neutrophil LFA-1 (CD11a) and Mac-1 (CD11b) expression}

Several specific alterations in neutrophil cell surface receptor expression levels of integrins over time were identified. Histograms and two-dimensional plots at different time points of all individual samples were analysed, showing increased circulatory neutrophil heterogeneity upon intervention. This contrasts with the control group, which exhibited a homogeneous neutrophil population consisting of

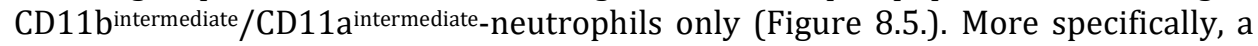
novel CD11 $b^{\text {high }} / \mathrm{CD} 11 \mathrm{a}^{\text {high }}$-neutrophil subtype appeared in circulation on postoperative day 7 (Figure 8.5./8.6.). This neutrophil subset was encountered in five of six animals in the day 7 group, exhibiting a statistically significant fraction (average $7.62 \%$, range: 5.56-9.24\%, $\mathrm{p}<0.01$ ) of the overall circulatory neutrophil composition (Figure 8.6.). This subset was further characterized by a statistically significant increased co-expression of CD49d (VLA-4) on the cell surface compared with other regular neutrophils $(\mathrm{p}<0.01)$. A back-gating analysis of the novel CD11 $\mathrm{b}^{\text {high }} / \mathrm{CD} 11 \mathrm{a}^{\text {high }}$-neutrophil population showed that all cells were spread across the neutrophil gate with respect to forward and side-scatter profiles. Finally, no 
differences in L-selectin co-expression between CD11 $b^{\text {high }} /$ CD11 $a^{\text {high }}$-neutrophils and regular neutrophils were seen (Figure 8.7.).
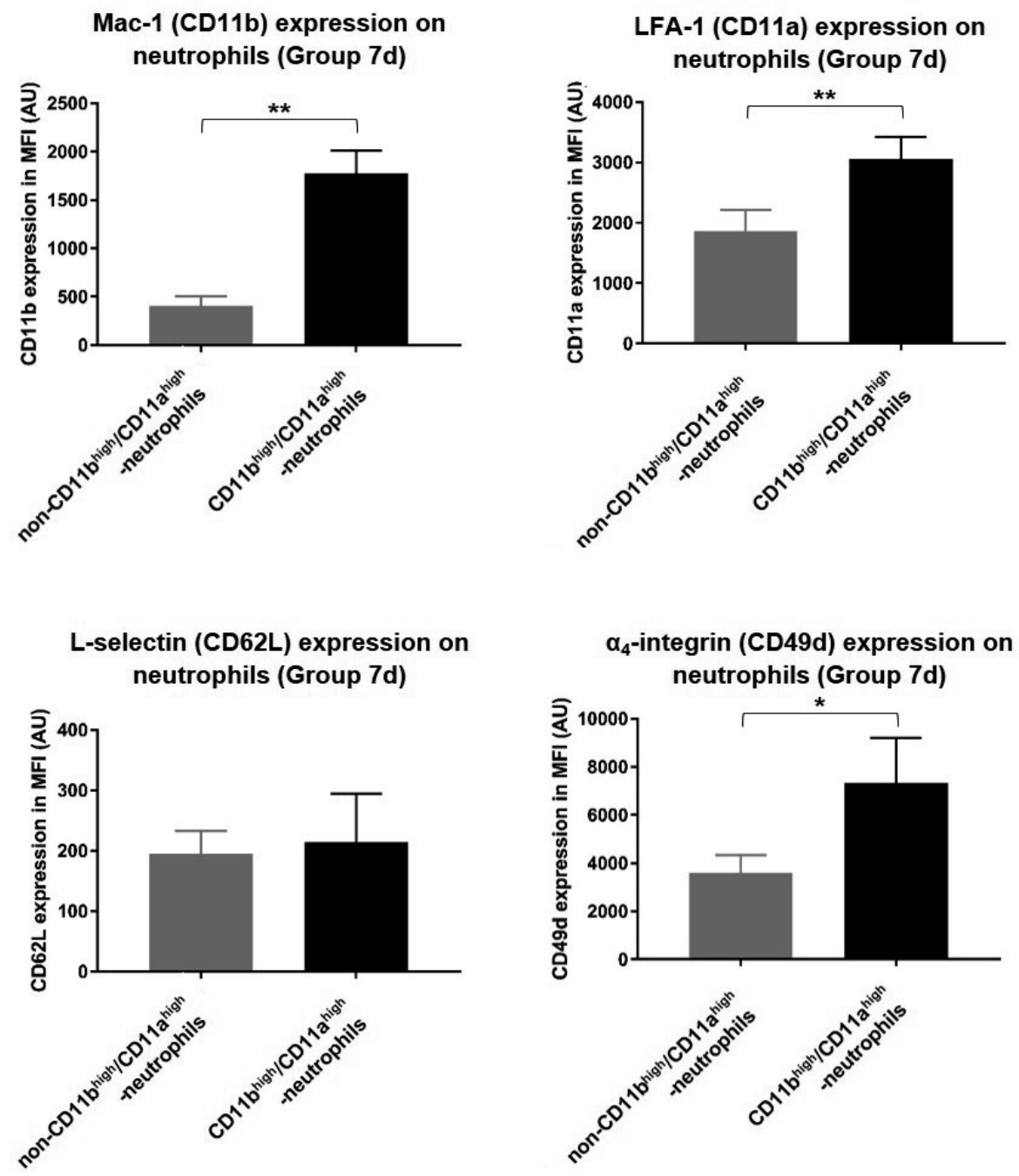

Figure 8.7. Differences in cell surface co-expression of L-selectin (CD62L) and $\alpha_{4}$-integrin (CD49d) on CD11 bigh $/$ CD11 $\mathrm{a}^{\text {high }}$ vs. regular neutrophil subtypes. The grey bars represent co-expression of cell surface receptors on regular, non-CD11 $\mathrm{b}^{\text {high }} / \mathrm{CD} 11 \mathrm{a}^{\text {high }}$-neutrophils, and the black bars represent coexpression of cell surface receptors on novel CD11 $\mathrm{b}^{\text {high }} / \mathrm{CD} 11 \mathrm{a}^{\text {high }}$-neutrophils in Group $7 \mathrm{~d}(\mathrm{n}=5)$. MFI: mean fluorescence intensity, AU: arbitrary units. Statistical significance ${ }^{*} \mathrm{p}<0.05,{ }^{* *} \mathrm{p}<0.01$.

\subsection{DISCUSSION}

Intramedullary nailing of long bone fractures represents a standard procedure in femoral shaft fractures and is performed 100,000-fold worldwide. Both the fracture itself and the surgical procedure for stabilization have been shown to be associated 
with inflammatory changes, which are necessary for the normal fracture healing process. On the other hand, if these inflammatory changes are dysregulated, local and/or systemic hyper-inflammatory conditions can evolve, causing local and/or systemic complications, such as non-unions and acute respiratory distress syndrome (ARDS) 23,24. Because neutrophils are the most abundant immune cells in the circulation and the primary first responders to trauma ${ }^{25,26}$, a dysregulation of the neutrophil response plays a key role in the pathogenesis of these complications $1,10,27$. Indeed, severe trauma leads to changes in neutrophil counts and functionality 28; however, research on the influence of isolated trauma (i.e., fracture) on circulatory neutrophils is scarce and ambiguous 3,4. Especially, influences on the long-term course have not been investigated until now. Therefore, it is important to gain knowledge on the time course and the specific pattern of neutrophil surface receptor expression alterations in the circulatory neutrophil pool during the post-traumatic process.

The present study focused on the circulatory neutrophil pool, and we were the first to describe changes in the circulatory neutrophil population in a standardized longterm observation fracture model, primarily focusing on the later inflammatory phase ( $>48$ hours after trauma) rather than the early/instant immune response. The results of the current study can be summarized as follows:

1. The number and activation of circulatory neutrophils is temporarily decreased at 3 days after femoral fracture and intramedullary nailing.

2. During the subsequent restoration of neutrophil homeostasis, the heterogeneity of the circulatory neutrophil pool increases. In this context, a new subset of CD11 $b^{\text {high }} /$ CD11 $\mathrm{a}^{\text {high }}$-neutrophils with co-expression of CD49d (VLA-4) occurs in systemic circulation at day 7 .

One potential theory to explain this decrease in the number and activation of circulatory neutrophils 3 days after the described procedure is that homing of neutrophils into the fracture hematoma occurs. From the literature, it is known that in the very early phases of the inflammatory stage of fracture healing, the neutrophil concentrations in the circulation and within the fracture hematoma remain equal for the first few hours 29,30 . However, within the first 24 hours after fracture, because of the release of mitochondrial DAMPs (damage-associated molecular patterns) and the subsequent release of different chemo-attractants in the surrounding tissue, the homing of circulatory neutrophils into the fracture hematoma occurs, which are later replaced by macrophages 3,31 . Corresponding to our study, Grøgaard et al. find a depletion of the circulatory neutrophils in the first 2 to 5 days after fracture, demonstrating a correlation of this depletion with an enhanced fracture healing of a femur osteotomy in rats ${ }^{4}$. This indicates, in accordance with other experimental rodent fracture studies, that accurate neutrophil recruitment and function in the fracture hematoma is crucial to initiate and coordinate the downstream responses leading to bone regeneration ${ }^{3,6}$. The importance of neutrophil homing to the fracture hematoma is also demonstrated in the clinical situation in the study of Bastian et al., in which serum from human fracture hematoma significantly generates neutrophil chemotaxis during the inflammatory stage of fracture healing ${ }^{1}$. Besides the extent of 
the inflammatory response, its duration and the insufficient termination (hyperactivated neutrophils) can impair the fracture healing process $2,6,32$.

In addition to the aforementioned recruitment into the fracture site, an enhanced migration of neutrophils into other organ systems after long bone fractures and intramedullary stabilization has also been described. In this context, the lungs have been identified as a primary target organ, in which neutrophils potentially damage parenchymal lung tissue ${ }^{7}$ and cause complications such as acute lung injury and ARDS 8,9. Clinical and experimental trauma studies have demonstrated that this pulmonary neutrophil infiltration peaks at 72 hours after fracture fixation ${ }^{8,33}$. This could be a second explanation for our discovered decrease of circulatory neutrophils.

Besides neutrophil count, their functionality is also of the utmost importance to assess the competence of blood neutrophils. Neutrophil functionality is regulated by cell membrane receptor expression and is a complex, multistage process ${ }^{34}$. These cell membrane receptors, which can detect different chemo-attractants (e.g., chemokines, complement) ${ }^{35}$, define different neutrophil subsets or phenotypes.

Among the neutrophil surface receptors, selectins and integrins are especially considered as essential for neutrophil activation and tissue migration. In the present study, the neutrophil surface expression of integrins and selectins was investigated for the first time in an experimental model of an isolated intramedullary stabilized femoral fracture. Previous studies focusing on the heterogeneity and function of circulatory neutrophils mainly focused on either the postoperative setting or on multiple trauma and found highly divergent results.

The current study showed no significant differences in L-selectin (CD62L) surface expression levels compared with the control conditions. Similar findings can be found in clinical studies focusing on patients after elective surgery. After cardiac surgery, no significant shift in L-selectin expression was found in the first 5 days postoperatively ${ }^{36}$. Also, Mommsen et al. find no significant difference in L-selectin expression of isolated neutrophils in the first 48 hours after elective lower limb surgery compared with preoperative expression levels. However, after stimulation with TNF- $\alpha$, they show that there is a significant decrease of L-selectin expression in the first 24 hours after surgery, indicating a role for the pro-inflammatory cytokine TNF- $\alpha$ in the neutrophil surface expression of L-selectin ${ }^{37}$. After multiple trauma, in contrast to our findings, Seekamp et al. find a significantly reduced level of L-selectin expression in the early phases (up to 24 hours) after trauma. This reduction is associated with the complicated post-traumatic course (i.e., the development of multi-organ dysfunction syndrome (MODS)) ${ }^{38}$. Besides the differences of the underlying insults, time-related differences of the sample collection might explain the aforementioned differences. In this context, it is known that L-selectin mediates the very early stages (24-48 hours after trauma) of the adhesion of neutrophils to endothelial cells. Therefore, we might have missed the early alterations in L-selectin expression in our study with the first time point being at 3 days after trauma 37-39. For the current experiment only female animals have been utilized. This is of specific interest as L-selectin, in contrast to other receptors, responses in trauma are believed to be gender specific. Interestingly, in accordance with a study from Van Griensven et al on gender related dimorphism in L-selectin receptor responses on circulatory PMNs in trauma, we also found an initial drop of L-selectin expression 
upon insult and later restoration to baseline levels 40 . This underlines the translational value of our model as well as the feasibility of our long-term fracture model in rats to perform proof-of-principle studies to test future immunomodulatory interventions. Future studies should focus on the occurrence of gender specific Lselectin responses upon trauma in rats as well.

In contrast to L-selectin, we demonstrated a relevant decrease of neutrophil CD11b expression 3 days after fracture induction. Thereafter, CD11b expression gradually increased for over 2 weeks after trauma. This recovery curve implies that restoration of CD11b-expression on the circulatory neutrophil pool takes at least 14 days. The results from a clinical study by Baehl et al. support our experimental findings, demonstrating that an isolated fracture is associated with reduced neutrophil cell surface expression of different integrins, such as CD11b, which are involved in binding and chemotaxis of neutrophils in the early phases after fracture. This decrease was followed by a gradual increase over 6 months of follow-up observation 41. A decrease of CD11b expression in the initial phase after trauma has also been encountered in other clinical studies ${ }^{42,43}$. In two clinical studies of severely injured patients, an initially decreased peripheral neutrophil-CD11b expression after three days followed by a gradual increase is found, which is in line with our results ${ }^{43}$.

We hypothesize that a transient decrease in neutrophil-CD11b expression is caused

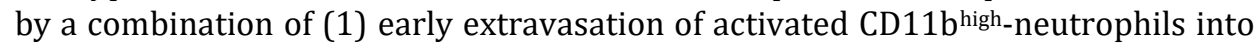
different tissue compartments (e.g., fracture and lung) directly after trauma and (2) an inadequate supply of new potent neutrophils in the circulation.

The current investigation also showed that neutrophil-CD11a surface expression levels changed over time after fracture induction and intramedullary stabilization. In previous studies, the exact expression pattern of CD11a has been partly controversially discussed, mainly in clinical trauma studies. Our results of an increase in CD11a-expression on day 3 after fracture induction are supported by the findings of Maekawa et al., which also show an increased expression from 3 hours up to 96 hours after trauma. On the other hand, our results do not correspond with the findings from a clinical study in which no significant increase of CD11a expression was demonstrated at 3 days after severe trauma ${ }^{43}$. Lo et al. even find a downregulation of CD11a expression although early at 24 hours after trauma ${ }^{44}$. Although based on our results we cannot comment on the early data from Lo et al., both CD11a and CD11b exhibit transient changes in adhesion- and migrationpromoting activity. Because our first measuring time point was 3 days, we might have missed an early downregulation of CD11a expression.

The aforementioned results of our and previous studies underline the results of a review of Mortaz et al. that focuses on neutrophil phenotyping in trauma patients. Here, specific post-traumatic expression patterns with various dynamics for surface receptors of neutrophils are described. In line with our results, the study shows that neutrophil CD11a and CD11b cell membrane expression levels do not rise synchronously in response to a traumatic insult, suggesting the existence of independent integrin regulation pathways ${ }^{43,44}$. Both integrins, CD11a and CD11b, play a prominent role in regulating the trans-endothelial migration of neutrophils, which is essential for an adequate immune response. In this migration cascade, these 
integrins partially have distinct roles $45-47$, in that CD11a is critical at each step of the neutrophil extravasation and that $\mathrm{CD} 11 \mathrm{~b}$ is more involved in the adhesion of neutrophils to the end othelial cells ${ }^{48}$. However, there seems to be an interplay, even an overlap, between these two integrins because they share the same heterodimeric component CD18 (integrin $\beta-2$ ) and partially bind to the same ligands ${ }^{39,48}$. In this context, as an expression of this connection between both integrins, we were the first to identify a new subset of CD11 $\mathrm{b}^{\text {high }} / \mathrm{CD} 11 \mathrm{a}^{\text {high }}$-neutrophils in the circulation at day 7.

Because of the late post-traumatic appearance in peripheral circulation more than 3 days after the insult, these neutrophils exhibit a unique pattern of mobilization compared with the other subtypes. Therefore, it has been suggested that they also might play a role in the regulation of the anti-inflammatory phase following trauma. Because neutrophils of this subset show a significantly higher co-expression with very late antigen-4 (VLA-4/CD49d) than regular neutrophils, it is most likely that they represent a unique phenotype.

The expression of CD49d (VLA-4) has not been reported on circulating neutrophils under homeostatic conditions ${ }^{49}$. On the contrary, CD49d expression has been identified on neutrophil progenitor cells in the bone marrow; therefore, it is tempting to hypothesize that these CD11 $\mathrm{b}^{\text {high }} / \mathrm{CD} 11 \mathrm{a}^{\text {high }}$-neutrophils are released from the bone marrow 49,50. CD49d (VLA-4) might play a significant role for the tissue migration of the new subset of neutrophils because it has been found to play a role in CD11b-independent neutrophil-adhesion pathways 51,52. Although it is not clear yet if these subsets definitively belong to separate developing lineages or embody certain activation states of a common precursor, the origin, characteristics, and functionality of the specific CD11 $\mathrm{b}^{\text {high }} / \mathrm{CD} 11 \mathrm{a}^{\text {high }}$ - neutrophil subset should be the focus of further research.

The current study also has some limitations. First, because our findings were derived from an animal model, they are not directly translatable to humans. We chose a rat model because this animal fracture model was established in our department, and it is suitable for this kind of research. Second, we used only one fixation method for the femur fractures, that is, intramedullary nailing; therefore, we cannot draw conclusions for other fixation methods based on these study results. Third, we planned to compare the data from the circulatory neutrophil pool with the neutrophils within the fracture hematoma, but we could not obtain enough fracture hematoma from the fracture site. Therefore, because the current descriptive study focused on the number and activation of neutrophils in the circulatory pool only, we could not make assertions about the functional relevance of the new subset of CD11 $b^{\text {high }} / C D 11 a^{\text {high }}$-neutrophils or their morphological characteristics yet. These important factors are planned to be addressed in subsequent studies in a larger animal model. Fifth, our study design does not enable the usage of statistical testing by repeated measure methods as all experimental animals have been terminated after sampling. In fact, as a total of $2 \mathrm{ml}$ blood per sample was required, we were not allowed to sample four times and thereby we would not have been able to determine long-term kinetics. To overcome this issue, we decided to perform termination after sampling and to group animal according to termination time point. By doing so we avoided potential confounding of the impact of withdrawal of relevant amounts of blood from rodents on metabolic 53 , hormonal 54 and even immunological 55 
homeostasis. In addition, we preferred to use female rats only, as they have relatively stable weight alterations in comparison with their male counterparts. As a consequence, extrapolation to the male situation in trauma is limited. Especially, as gender specific cellular immune response, and more specifically, L-selectin alterations upon severe trauma have been reported by Van Griensven et al. ${ }^{40}$.

\subsection{CONCLUSIONS}

This descriptive, small animal fracture study is the first to show that an intramedullary stabilized femur fracture is associated with a temporary reduction in circulatory neutrophil count and concurrent changes in circulatory neutrophil function. Moreover, we demonstrated that the restoration to homeostatic neutrophil activation status occurs concomitantly with an increased heterogeneity of the circulatory neutrophil pool, which is accentuated by the appearance of a novel neutrophil subtype (CD11 $\mathrm{b}^{\text {high }} / \mathrm{CD} 11 \mathrm{a}^{\text {high }}$ ). Improved knowledge of neutrophil count and functionality after trauma form an excellent basis for future studies to further elucidate the role of neutrophils as activators and regulators of different posttraumatic processes, potentially resulting in local (e.g., fracture healing disturbances) or systemic (e.g., MODS) complications. This might result in the development of specific therapies to reduce adverse outcomes after trauma.

\subsection{ACKNOWLEDGEMENTS}

Ethics approval and consent to participate. All methods were performed with the approval of the institutional animal committee and of the Governmental Animal Care and Use Committee (Landesamt für Natur, Umwelt und Verbraucherschutz, North Rhine-Westphalia, Recklinghausen, Germany; Protocol No. 84-02.04.2015.A078). All animal experiments were performed in accordance with the guidelines and regulations of the Federation of European Laboratory Animal Science Associations (FELASA) and the German Society of Laboratory Animals.

Consent for publication. Not applicable.

Availability of data and material. The datasets used and/or analysed during the current study are available from the corresponding author on reasonable request.

Competing interests. The authors declare that they have no financial and/or nonfinancial competing interests.

Funding. This research project was supported by the START-Program of the Faculty of Medicine, RWTH Aachen, Germany (Grant nr. 691451). The sponsor had no role in the design of this study; collection, analysis, and interpretation of data; writing of the manuscript; or decision to submit the article for publication.

Author contributions. Michel P.J. Teuben: Conceptualization, methodology, validation, investigation, resources, data curation, writing - original draft, visualization. Martijn Hofman: Conceptualization, methodology, validation, investigation, resources, data curation, writing - original draft, project administration, funding acquisition. Johannes Greven: Conceptualization, methodology, validation, investigation, resources, project administration. Alba 
Shehu: Conceptualization, investigation, data curation, visualization. Henrik Teuber: Conceptualization, investigation, data curation, visualization. Roman Pfeifer: Conceptualization, writing - review and editing, supervision. Hans-Christoph Pape: Conceptualization, writing - review and editing, supervision. Frank Hildebrand: Conceptualization, writing - review and editing, supervision. All authors granted final approval of the submitted version.

\section{Proofreading. Scribendi Inc. (Chatham, Ontario N7M 0N3 Canada) performed the proofreading of our manuscript.}

\subsection{REFERENCES}

1. Bastian OW, Mrozek MH, Raaben M, Leenen LPH, Koenderman L, Blokhuis TJ. Serum from the Human Fracture Hematoma Contains a Potent Inducer of Neutrophil Chemotaxis. Inflammation 2018; 41(3): 1084-92.

2. Claes L, Recknagel S, Ignatius A. Fracture healing under healthy and inflammatory conditions. Nature reviews Rheumatology 2012; 8(3): 133-43.

3. Chung R, Cool JC, Scherer MA, Foster BK, Xian CJ. Roles of neutrophil-mediated inflammatory response in the bony repair of injured growth plate cartilage in young rats. Journal of leukocyte biology 2006; 80(6): 1272-80.

4. Grogaard B, Gerdin B, Reikeras 0 . The polymorphonuclear leukocyte: has it a role in fracture healing? Archives of orthopaedic and trauma surgery 1990; 109(5): 268-71.

5. Bastian OW, Koenderman L, Alblas J, Leenen LP, Blokhuis TJ. Neutrophils contribute to fracture healing by synthesizing fibronectin+ extracellular matrix rapidly after injury. Clinical immunology 2016; 164: 78-84.

6. Kovtun A, Bergdolt S, Wiegner R, Radermacher P, Huber-Lang $M$, Ignatius $A$. The crucial role of neutrophil granulocytes in bone fracture healing. European cells \& materials 2016; 32: 152-62.

7. van Griensven M Krettek C, Pape H. Immune Reactions after Trauma. Eur J Trauma 2003; 29: 18192.

8. Störmann P WN, Köhler K, Auner B, Simon T.P. Pfeifer R, Horst K, Pape H.C. Hildebrand F, Wutzler S, Marzi I, Relja B. . Monotrauma is associated with enhanced remote inflammatory response and organ damage, while polytrauma intensifies both in porcine trauma model. European journal of trauma and emergency surgery: official publication of the European Trauma Society 2020; Feb(46(1)): 31-42.

9. Pape HC, Grimme K, Van Griensven M, et al. Impact of intramedullary instrumentation versus damage control for femoral fractures on immunoinflammatory parameters: prospective randomized analysis by the EPOFF Study Group. The Journal of trauma 2003; 55(1): 7-13.

10. Keel M, Trentz O. Pathophysiology of polytrauma. Injury 2005; 36(6): 691-709.

11. Dunne JL, Collins RG, Beaudet AL, Ballantyne CM, Ley K. Mac-1, but not LFA-1, uses intercellular adhesion molecule- 1 to mediate slow leukocyte rolling in TNF-alpha-induced inflammation. Journal of immunology 2003; 171(11): 6105-11.
12. Morton DB, Griffiths PH. Guidelines on the recognition of pain, distress and discomfort in experimental animals and an hypothesis for assessment. The Veterinary record 1985; 116(16): 431-6.

13. Hickman DL, Swan M. Use of a body condition score technique to assess health status in a rat model of polycystic kidney disease. Journal of the American Association for Laboratory Animal Science : JAALAS 2010; 49(2): 155-9.

14. Becker JB, Arnold AP, Berkley KJ, et al. Strategies and methods for research on sex differences in brain and behavior. Endocrinology 2005; 146(4): 1650-73.

15. Milad MR, Igoe SA, Lebron-Milad K, Novales JE. Estrous cycle phase and gonadal hormones influence conditioned fear extinction. Neuroscience 2009; 164(3): 887-95.

16. Marcondes FK, Bianchi FJ, Tanno AP. Determination of the estrous cycle phases of rats: some helpful considerations. Brazilian journal of biology = Revista brasleira de biologia 2002; 62(4A): 609-14.

17. Kluter $\mathrm{T}$, Weuster $\mathrm{M}$, Bruggemann $\mathrm{S}$, et al. Rivaroxaban does not impair fracture healing in a rat femur fracture model: an experimental study. $B M C$ musculoskeletal disorders 2015; 16: 79.

18. Bonnarens F, Einhorn TA. Production of a standard closed fracture in laboratory animal bone. Journal of orthopaedic research : official publication of the Orthopaedic Research Society 1984; 2(1): 97-101.

19. Bolivar S, Anfossi R, Humeres C, et al. IFN-beta Plays Both Pro- and Anti-inflammatory Roles in the Rat Cardiac Fibroblast Through Differential STAT Protein Activation. Frontiers in pharmacology 2018; 9: 1368.

20. Vander Top EA, Perry GA, Gentry-Nielsen MJ. A novel flow cytometric assay for measurement of in vivo pulmonary neutrophil phagocytosis. $B M C$ microbiology 2006; 6: 61.

21. Skrajnar S, Anzur Lasnik M, Bedina Zavec A. A flow cytometric method for determination of the blood neutrophil fraction in rats. Journal of the American Association for Laboratory Animal Science : JAALAS 2009; 48(2): 152-6.

22. Barnett-Vanes A, Sharrock A, Birrell MA, Rankin S. A Single 9-Colour Flow Cytometric Method to Characterise Major Leukocyte Populations in the Rat: Validation in a Model of LPS-Induced Pulmonary Inflammation. PloS one 2016; 11(1): e0142520. 
23. Giannoudis PV, Pape HC, Cohen AP, Krettek C, Smith RM. Review: systemic effects of femoral nailing: from Kuntscher to the immune reactivity era. Clinical orthopaedics and related research 2002; (404): 37886.

24. Pape HC, Auf'm'Kolk M, Paffrath T, Regel G, Sturm JA, Tscherne H. Primary intramedullary femur fixation in multiple trauma patients with associated lung contusion--a cause of posttraumatic ARDS? The Journal of trauma 1993; 34(4): 540-7; discussion 7-8.

25. Botha AJ, Moore FA, Moore EE, Kim FJ, Banerjee A, Peterson VM. Postinjury neutrophil priming and activation: an early vulnerable window. Surgery 1995 ; 118(2): 358-64; discussion 64-5.

26. Botha AJ, Moore FA, Moore EE, Sauaia A, Banerjee A, Peterson VM. Early neutrophil sequestration after injury: a pathogenic mechanism for multiple organ failure. The Journal of trauma 1995; 39(3): 411-7.

27. Lord JM, Midwinter MJ, Chen YF, et al. The systemic immune response to trauma: an overview of pathophysiology and treatment. Lancet 2014; 384(9952): 1455-65.

28. Kovtun A, Messerer DAC, Scharffetter-Kochanek $\mathrm{K}$, Huber-Lang $\mathrm{M}$, Ignatius A. Neutrophils in Tissue Trauma of the Skin, Bone, and Lung: Two Sides of the Same Coin. Journal of immunology research 2018; 2018: 8173983.

29. Li H, Liu J, Yao J, Zhong J, Guo L, Sun T. Fracture initiates systemic inflammatory response syndrome through recruiting polymorphonuclear leucocytes. Immunologic research 2016; 64(4): 1053-9.

30. Schmidt-Bleek K, Schell H, Kolar P, et al. Cellular composition of the initial fracture hematoma compared to a muscle hematoma: a study in sheep. Journal of orthopaedic research : official publication of the Orthopaedic Research Society 2009; 27(9): 1147 51.

31. Andrew JG, Andrew SM, Freemont AJ, Marsh DR. Inflammatory cells in normal human fracture healing. Acta orthopaedica Scandinavica 1994; 65(4): 462-6.

32. Butterfield TA, Best TM, Merrick MA. The dual roles of neutrophils and macrophages in inflammation: a critical balance between tissue damage and repair. Journal of athletic training 2006; 41(4): 457-65.

33. Relja B, Taraki R, Teuben MP, et al. Sera from severe trauma patients with pneumonia and without infectious complications have differential effects on neutrophil biology. BMC pulmonary medicine 2016; 16(1): 171.

34. Seekamp A, van Griensven M, Dhondt E, et al. The effect of anti-L-selectin (aselizumab) in multiple traumatized patients--results of a phase II clinical trial. Critical care medicine 2004; 32(10): 2021-8.

35. Stillie R, Farooq SM, Gordon JR, Stadnyk AW. The functional significance behind expressing two IL-8 receptor types on PMN. Journal of leukocyte biology 2009; 86(3): 529-43.

36. Fung YL, Silliman CC, Minchinton RM, Wood P, Fraser JF. Cardiopulmonary bypass induces enduring alterations to host neutrophil physiology: a single- center longitudinal observational study. Shock 2008; 30(6): 642-8.

37. Mommsen P, Barkhausen T, Hildebrand F, Zeckey C, Krettek C, van Griensven M. Regulation of L-selectin expression by trauma-relevant cytokines. Pathology, research and practice 2011; 207(3): 142-7.

38. Seekamp A, van Griensven M, Hildebrandt F, Brauer N, Jochum M, Martin M. The effect of trauma on neutrophil L-selectin expression and sL-selectin serum levels. Shock 2001; 15(4): 254-60.

39. Mortaz E, Zadian SS, Shahir M, et al. Does Neutrophil Phenotype Predict the Survival of Trauma Patients? Frontiers in immunology 2019; 10: 2122.

40. van Griensven M, Barkhausen T, Hildebrand F, et al. L-selectin shows time and gender dependency in association with MODS. Injury 2004; 35(11): 1087-95.

41. Baehl S, Garneau H, Le Page A, et al. Altered neutrophil functions in elderly patients during a 6month follow-up period after a hip fracture. Experimental gerontology 2015; 65: 58-68.

42. Scannell G, Waxman K, Vaziri ND, et al. Effects of trauma on leukocyte intercellular adhesion molecule$1, \mathrm{CD} 11 \mathrm{~b}$, and CD18 expressions. The Journal of trauma 1995; 39(4): 641-4.

43. Botha AJ, Moore FA, Moore EE, Peterson VM, Goode AW. Base deficit after major trauma directly relates to neutrophil CD11b expression: a proposed mechanism of shock-induced organ injury. Intensive care medicine 1997; 23(5): 504-9.

44. Lo SK, Van Seventer GA, Levin SM, Wright SD. Two leukocyte receptors (CD11a/CD18 and CD11b/CD18) mediate transient adhesion to endothelium by binding to different ligands. Journal of immunology 1989; 143(10): 3325-9.

45. Ding ZM, Babensee JE, Simon SI, et al. Relative contribution of LFA-1 and Mac-1 to neutrophil adhesion and migration. Journal of immunology 1999; 163(9): 5029-38.

46. Heit B, Colarusso P, Kubes P. Fundamentally different roles for LFA-1, Mac-1 and alpha4-integrin in neutrophil chemotaxis. Journal of cell science 2005; 118(Pt 22): 5205-20.

47. Li N, Mao D, Lu S, Tong C, Zhang Y, Long M. Distinct binding affinities of Mac-1 and LFA-1 in neutrophil activation. Journal of immunology 2013; 190(8): 4371-81.

48. Hyun YM, Choe YH, Park SA, Kim M. LFA-1 (CD11a/CD18) and Mac-1 (CD11b/CD18) distinctly regulate neutrophil extravasation through hotspots I and II. Experimental \& molecular medicine 2019; 51(4): 1-13.

49. Lund-Johansen F, Terstappen LW. Differential surface expression of cell adhesion molecules during granulocyte maturation. Journal of leukocyte biology 1993; 54(1): 47-55.

50. Soligo D, Schiro R, Luksch R, et al. Expression of integrins in human bone marrow. British journal of haematology 1990; 76(3): 323-32.

51. Harlan JM. Leukocyte adhesion deficiency syndrome: insights into the molecular basis of 


\section{CHAPTER 8}

leukocyte emigration. Clinical immunology and immunopathology 1993; 67(3 Pt 2): S16-24.

52. Reinhardt PH, Elliott JF, Kubes P. Neutrophils can adhere via alpha4beta1-integrin under flow conditions. Blood 1997; 89(10): 3837-46.

53. Arnold M, Langhans W. Effects of anesthesia and blood sampling techniques on plasma metabolites and corticosterone in the rat. Physiology \& behavior 2010; 99(5): 592-8.

54. Rao RH. Changes in insulin sensitivity from stress during repetitive sampling in anesthetized rats. The American journal of physiology 1992; 262(6 Pt 2): R1033-9.

55. Boudesco C, Rattier T, Garrido C, Jego G. Do not stress, just differentiate: role of stress proteins in hematopoiesis. Cell death \& disease 2015; 6: e1628.

56. McManus AT. Examination of neutrophil function in a rat model of decreased host resistance following burn trauma. Rev Infect Dis. 1983;5 Suppl 5:S898S907. doi:10.1093/clinids/5.supplement_5.s898 


\title{
The impact of intramedullary nailing on the characteristics of the pulmonary neutrophil pool in rodents.
}

\section{International Orthopaedics}

2020 Mar;44(3):595-602. doi: 10.1007/s00264-019-04419-6. [Epub 2020 Jan 3] PMID: 31900572

\author{
Michel P.J. Teuben ${ }^{1,2}$ \\ Martijn Hofman ${ }^{3}$ \\ Alba Shehu ${ }^{3}$ \\ Johannes Greven ${ }^{3}$ \\ Zhi Qiao ${ }^{3}$ \\ Kai Oliver Jensen ${ }^{1,2}$ \\ Frank Hildebrand ${ }^{3}$ \\ Roman Pfeifer ${ }^{1,2}$ \\ Hans Christoph Pape ${ }^{1,2}$
}

1 Department of Traumatology, University Hospital Zürich, Switzerland

2 Harald Tscherne Research Laboratory, University Hospital Zürich, Switzerland

3 Department of Orthopaedic Trauma and Reconstructive Surgery, University Medical Center RWTH Aachen, Germany 


\begin{abstract}
Purpose. Dysregulation of polymorphonuclear neutrophil (PMN) biology is associated with the development of inflammatory complications after trauma, such as acute respiratory distress syndrome (ARDS). Data about the influence of surgical procedures, such as intramedullary nailing (IMN) is sparse. This study aimed to characterize the remote neutrophil response in the lungs in case of a femur fracture and intramedullary nailing.
\end{abstract}

Methods. A standardized rat model including intramedullary nailing and a femur fracture was utilized. Groups were terminated after observation times of 3, 7 and 14 days. Neutrophils were isolated from lung parenchyma and broncho-alveolar lavage fluid (BALF) and analysed by flow cytometry. Absolute neutrophil numbers as well as membrane expression levels of CD11b, CD62L and CD11a were compared.

Results. Pulmonary neutrophil numbers were increased 3 days after intervention. Membrane expression levels of CD11b $(\mathrm{P}<0.01)$, CD62L $(\mathrm{P}<0.01)$ and CD11a $(\mathrm{p}=0.06)$ on parenchymal PMNs increased as well after 3 days. Thereafter values restored gradually to physiological levels. Furthermore, neutrophil activation status between parenchymal and BALF-PMN pools did not correlate.

Conclusions. The current study demonstrates that IMN and a femur fracture are associated with transient increased pulmonary PMN deposition, as well as a specific neutrophil activation pattern characterized by temporary increased selectin and integrin receptor expression on pulmonary neutrophils. This phenomenon might play an important role in the pathomechanism of ARDS after trauma. Moreover, we found striking differences between parenchymal and BALF-neutrophil populations, demonstrating the limited readout potential of BALF-analysis to investigate the entire pulmonary neutrophil pool. 


\subsection{INTRODUCTION}

Pulmonary complications such as acute lung injury (ALI) and adult respiratory distress syndrome (ARDS) occur after femoral fractures and intramedullary nailing (IMN). Additionally, a pulmonary entity called 'fat embolism syndrome' (FES) has been described as well. This syndrome is difficult to distinguish from ALI/ARDS and also occurs in septic patients ${ }^{1-3}$. Current concepts emphasize that femoral fracture hematomas can alter, and more specifically, increase systemic levels of inflammatory mediators. These mediators could also activate circulating polymorphonuclear neutrophils (PMNs), that have the potency to damage parenchymal lung tissue upon extravasation and subsequent activation when residing in the lungs 4,5 . It has well been demonstrated that systemic neutrophil dysregulation is an important process in the development of ARDS ${ }^{6,7}$. Histological studies after severe experimental trauma, and on patients who died from ARDS revealed substantial increased pulmonary neutrophil influx ${ }^{8-10}$. Currently, the pathogenesis of these complications and their specific relation with IMN are unclear, but there appears to be a role for pulmonary polymorphonuclear neutrophils.

Cell labelling studies performed by Wintrobe's group established the concept of increased neutrophil deposition being associated with pulmonary inflammatory pathologies. In their studies on healthy individuals they demonstrated that only half of DFP32-labelled granulocytes, that were injected to volunteers, were later encountered in peripheral blood. These findings suggested that the non-circulating cells migrated instantly into the tissue compartment or adhered to a vessel wall (marginated pool) ${ }^{11}$. With the development of $\ln 111$-cell labelling techniques, margination processes could be imaged more precisely. Animal experiments revealed that the pulmonary vascular bed was an important site of neutrophil margination. However, as a result of further studies in which labelling conditions of previous experiments were critically examined, it became clear that the observed effects did not reflect pure physiological conditions, as some form of injury/insult was implemented in the utilized models ${ }^{12}$. Circumstances in which neutrophil transit time in the lungs is prolonged include several pathologies as ALI/ARDS and fat embolism syndrome, and this affects pulmonary neutrophil deposition 11,13,14. According to experimental trauma studies, pulmonary PMN infiltration peaks at 72 hours after trauma ${ }^{15}$. However, the characteristics (and specific alterations over time) of pulmonary PMNs upon trauma are unclear.

Pulmonary tissue is not homogeneous and several compartments, namely lung parenchyma and the broncho-alveolar compartment, can be identified ${ }^{16}$. Subsequently, neutrophils are situated in different lung compartments as well and different pulmonary neutrophil pools can be distinguished. In addition, large pools of neutrophils appear to be marginated to the vascular wall, and the lung neutrophil population consists, under homeostatic conditions, mainly of cells that adhere to the endothelium of pulmonary blood vessels ${ }^{17}$. Upon activation, these marginated neutrophils can undergo rapid trans-endothelial and trans-epithelial migration into the interstitium and alveolar spaces. The insult-evoked influx of these cells into both lung parenchyma and broncho-alveolar spaces might alter the constitution and characteristics of the neutrophil pools in these compartments ${ }^{18}$. This process might play a relatively unexplored role in the development of inflammatory complications in trauma. 
Bronchoalveolar lavage was proposed as early as 1995 as a procedure to diagnose fat embolism syndrome. During this lavage, PMNs could be obtained for analysis ${ }^{19,20}$. In 2010, Blankstein described the combination of haemorrhagic shock, resuscitation, and fat embolism syndrome elicited neutrophil activation, infiltration of alveoli by PMNs, and inflammatory cytokine expression in bronchoalveolar lavage fluid 5 . However, specific characteristics of neutrophils residing in different neutrophil compartments after trauma, as well as alterations over time have not been studied yet. Pulmonary neutrophil homing is a multistep process, orchestrated by alterations in cell-surface expression and affinity of mainly selectins and integrins. In order to obtain more insight into the pathogenesis of trauma-induced ARDS, we defined the following hypotheses:

(1) Intramedullary nailing and a unilateral femur fracture is associated with the expansion of the pulmonary neutrophil population and increased activation of both the parenchymal neutrophil pool and the broncho-alveolar neutrophil pool.

(2) Cell-surface expression of activation markers on PMNs differs between distinct lung compartments (parenchymal vs. bronco-alveolar space).

\subsection{MATERIALS AND METHODS}

\subsubsection{Ethical approval}

Prior to the start of the experiments the protocol was approved by the institutional animal committee and of the regulating authority: Landesamt für Natur, Umwelt und Verbraucherschutz (LANUV) Nordrhein-Westfalen, Recklinghausen, Deutschland (permit AZ 84-02.04.2015.A078). The utilized experimental animals are a part of a larger rodent study.

\subsubsection{Experimental model}

Adult female Sprague-Dawley rats (Harlan Industries, Indianapolis, Indiana, USA, 250350 grams) were subjected to standardized intramedullary nailing and a unilateral femur fracture, as previously described ${ }^{21,22}$. Subcutaneous premedication included: $0.03 \mathrm{mg} / \mathrm{kg}$ buprenorphine hydrochloride (Reckitt Benckiser Healthcare Ltd., United Kingdom). General anaesthesia was induced by 100 mg/kg Ketamine (Pfizer, New York, USA) intraperitoneally and 2\% $10 \mathrm{mg} / \mathrm{kg}$ Xylazine (Xylapan, Vetoquinol, Ravensburg, Germany) intraperitoneally. Anaesthesia was maintained by $2-2.5 \%$ Isoflurane inhalation. Postoperative animals received buprenorphine hydrochloride twice a day.

\subsubsection{Study groups}

Animals were sacrificed after 3, 7 and 14 days of observation $(\mathrm{N}=6)$. In addition, one control group was added to define homeostatic values $(\mathrm{N}=3)$. Animals were randomized for groups and terminated by cervical dislocation under isoflurane anaesthesia. The following groups were included:

Study group 1 : Intramedullary nailing + unilateral femur fracture, observation period: 3 days.

Study group 2 : Intramedullary nailing + unilateral femur fracture, observation period: 7 days.

Study group 3 : Intramedullary nailing + unilateral femur fracture, observation period: 14 days.

Control group : anaesthesia (in line with study groups) and direct termination. 


\subsubsection{Isolation of single-cell solutions from different pulmonary compartments}

Directly after termination a thoracotomy was performed and lungs (including trachea) were isolated for further analysis. First, the left main bronchus was clipped and a blunt syringe was utilized to flush the right lung. A total of three flushes with each $1 \mathrm{~mL}$ of icecold phosphate buffered saline (PBS) were performed. The solution was then filtered using a $100 \mu$ l cell strainer (BALF-single cell solution).

Thereafter the right caudal lobe was isolated. Lung tissue was crushed mechanically, and single cell solutions were collected in a $50 \mathrm{~mL}$ Falcon-tube (lung parenchymal single cell solution). Then samples were lysed using a Red blood cell (RBC)-lysing buffer. After this lysis step, FACS-Buffer (phosphate buffered saline enriched with $0.5 \%$ bovine serum albumin and $0.5 \mathrm{mM}$ EDTA) was added to both samples and two washing steps were performed. Thereafter conjugated antibodies were added and allowed to incubate for 45 minutes. After staining the single cell solutions were washed twice and directly analysed by flow cytometry (within 1 hour). By doing so, neutrophil populations were isolated from two distinct compartments of uninjured lungs:

1. Lung parenchymal neutrophils and,

2. Broncho-alveolar neutrophils.

\subsubsection{Flow cytometry analysis and pulmonary neutrophil identification}

Neutrophils have been implicated as having a pivotal role in the development of acute lung injury. Neutrophils were identified and differentiated from other white blood cells by characteristic CD 45 and RP-1 fluorescence and light scatter properties as measured by flow cytometry. The following gating strategy was utilized: isolation of viable leukocytes (CD45 high), exclusion of doublets, inclusion of RP1 ${ }^{\text {high }} / \mathrm{SSC}$ high cells. From each sample a minimum of 10.000 RP1-positive cells were analysed. This protocol has been validated by pilot experiments and fluorescence levels were compared to negative control values as well as with fluorescence levels on blood and bone marrow cells. In order to investigate characteristics of different pulmonary neutrophil populations and changes over time, membrane receptor expression levels of activation markers: integrin Mac-1 (CD11b), integrin LFA1 (CD11a) and L-selectin (CD62L) were measured. CD11b expression was used because it is an early indicator of the acute inflammatory reaction preceding lung injury. Moreover, CD11b expression on circulating neutrophils increases after major trauma. As neutrophil transmigration to the site of inflammation is a multistep process and dependent on the expression of cell-surface glycoprotein adhesion molecules we also studied cell-surface receptor expression dynamics of LFA1 and L-selectin. Counting beads were added in order to calculate absolute cells counts of broncho-alveolar lavage fluid samples. Absolute neutrophil numbers and the neutrophil fraction were determined as described by Skrajnar et al. ${ }^{23}$. A Canto II-device (Becton \& Dickinson, Mountain View, CA, USA) and FACS Diva Software (Becton \& Dickinson, Mountain View, CA, USA) were utilized to analyse samples. In order to analyse differences between the tissue compartments we plotted correlations between all markers and calculated statistical significance. 


\subsubsection{Reagents}

RP-1 clone RUO (Becton \& Dickinson, Mountain View, CA, USA), CD11b clone M1/70 (eBioscience Vienna, Austria), CD62L clone OX-85 (AbD Serotec, Düsseldorf, Germany), CD11a clone WT.1 (AbD Serotec, Düsseldorf, Germany), CD45 clone 10558 (Abcam, Cambridge, Great Britain), CountBright counting beads (ThermoFisher Scientific, Waltham, United States). Phosphate buffered saline (PBS) (Sigma, Deisenhofen, Germany). RBC Lysis-Buffer (Bio-rad, Hercules, United States), FACS-Buffer (phosphate buffered saline enriched with $0.5 \%$ bovine serum albumin and $0.5 \mathrm{mM}$ Ethylenediaminetatraacetic acid (EDTA)).

\subsubsection{Statistical analysis}

Data are displayed as means and SEM, unless described otherwise. Groups were compared by using the Mann-Whitney U test. Differences were considered significant if $\mathrm{p}<0.05$. Data was analysed with the following software programs: SPSS version 20.0 (SPSS Inc., Chicago, IL, USA) and GraphPad Prism (GraphPad Software Inc., La Jolla, CA, USA).

\subsection{RESULTS}

All animals survived the observation period and no signs of infection were seen. Furthermore, all animals were able to mobilize within 24 hours after the intervention. Single cell solutions from both the parenchymal compartment and the broncho-alveolar compartment were prepared and analysed as described previously. Pulmonary neutrophil numbers/deposition was determined by analysis of bronchoalveolar fluid. Additionally, specific characteristics of both the parenchymal as well as the bronchoalveolar neutrophil populations were determined and compared.

\subsubsection{Intramedullary nailing is associated with temporarily increased pulmonary neutrophil deposition}

As displayed in figure 9.1.a, absolute neutrophil numbers in broncho-alveolar lavage samples were increased significantly 3 days post-insult (mean: 35,026 PMNs/ml). Neutrophil counts thereafter decreased and equalled those under control conditions after both 7 and 14 days. Furthermore, the percentage of PMNs out of total bronchoalveolar lavage fluid (BALF) leukocytes also peaked at 33.3\% of leukocytes in the 3-day observation group (Figure 9.1.b). This was

significantly higher than the percentages encountered under control conditions (mean PMN-fraction: 7.4\%, $\mathrm{p}=0.036$ ) and in both animals terminated at 7 and 14 days (respectively, mean PMN-fraction: $9.0 \%, \mathrm{p}<0.01$ and mean PMN fraction: $5.4 \% \mathrm{p}<$ 0.01).

\subsubsection{Alterations in activation status of the parenchymal neutrophil pool over time}

A statistically significant increase in CD11b expression on parenchymal neutrophils was observed after 72 hours of observation compared with control conditions ( $\mathrm{p}<$ 0.01) (Figure 9.2.a). Thereafter, PMN Mac1 expression on the parenchymal PMN pool decreased gradually over time. However, Mac-1 expression did not recover fully to baseline levels within the first 14 days after surgery $(\mathrm{p}<0.01)$. 


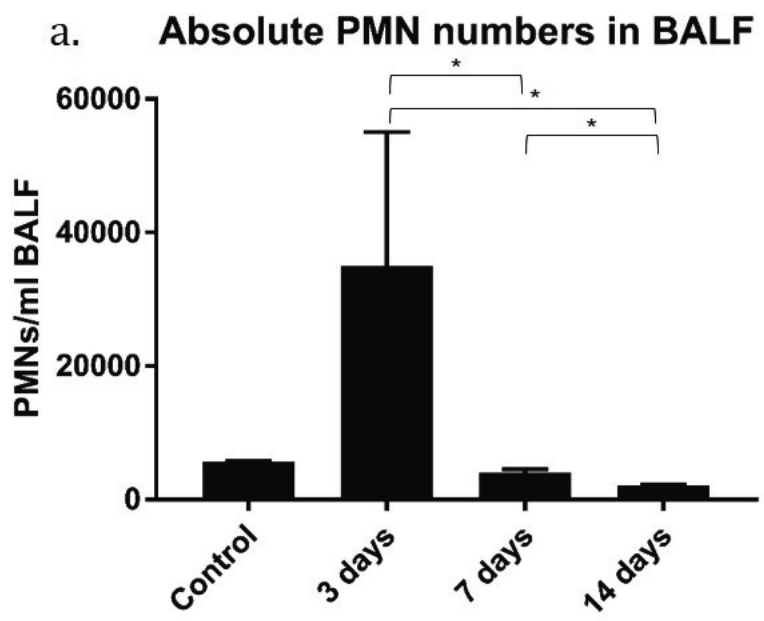

b. BALF Neutrophil fraction

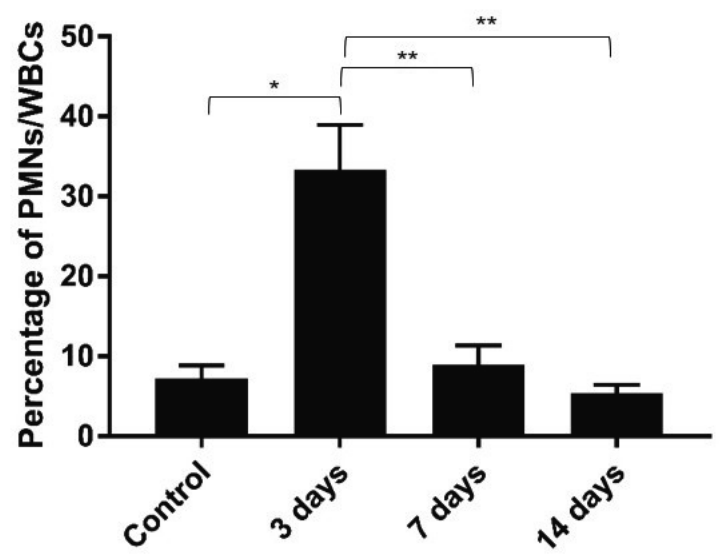

Figure 9.1. Changes in neutrophil fraction and absolute neutrophil numbers in BALF between groups. Variations in absolute neutrophil numbers in BALF (a) and in neutrophil fraction in BALF-analysis (b). Significance between groups is displayed as ${ }^{*} p<0.05,{ }^{* *} p<0.01$.

Membrane receptor expression of CD11a on parenchymal PMNs (Figure 9.2.b) did not change significantly over time. Nevertheless, a notable statistically non-significant trend towards temporary peaking of LFA1 expression levels at 3 days of observation was found $(p=0.061)$. Pulmonary neutrophil L-selectin expression (Figure 9.2.c) was significantly increased after 3 days of observation $(p<0.01)$. Neutrophil L-selectin expression levels thereafter returned to baseline levels, and no statistically significant difference between control conditions and the group observed for 14 days was encountered $(p=0.69)$. 


\subsubsection{Changes in cell-surface activation of the broncho-alveolar neutrophil pool following trauma}

In contrast to the observed alterations of PMN-CD11b expression levels in the parenchymal neutrophil pool, no statistically significant changes in BALF-PMN expression levels over time occurred (Figure 9.3.a). LFA-1 expression levels on broncho-alveolar neutrophils, on the other hand, were significantly lower at 3 days of observation compared with control conditions $(\mathrm{P}=0.02)$. However, at both 7 and 14 days of observation, LFA-1 cell-surface expression levels equalled those under control conditions again (Figure 9.3.b). As displayed in Figure 3c, no statistically significant alterations in PMN-CD62L expression over time on BALF neutrophils were seen.

\subsubsection{Activation status of parenchymal and broncho-alveolar neutrophils do not correlate when measured by flow cytometry}

We also tested for differences between the cell-surface expression levels of relevant neutrophil activation markers on both parenchymal and BALF neutrophils. When pooling all measurements, we found no statistically significant correlation of neutrophil CD11b ( $p=0.93)$, CD11a ( $p=0.43$ ) or CD62L ( $p=0.20)$ cell-surface expression levels between parenchymal PMNs and BALF neutrophils (Figure 9.4.).

\subsection{DISCUSSION}

Previous studies on the inflammatory cellular response after trauma focused mainly on alterations in blood neutrophils. However, circulatory neutrophils are, in contrast to tissue neutrophils, not believed to be harmful by themselves ${ }^{6,24}$. The current study is the first to determine the specific neutrophil response to intramedullary nailing in the pulmonary tissue compartment.

1. The current study demonstrates that intramedullary nailing and a unilateral fracture are associated with transient increases in pulmonary neutrophil deposition and increases in the activation status of the pulmonary neutrophil pool.

2. Moreover, this study revealed striking differences in the activation status of neutrophils belonging to the pulmonary parenchymal compartment and those belonging to the bronchoalveolar compartment and thereby demonstrates compartmentalization related to intrapulmonary neutrophil heterogeneity.

Dysregulation of neutrophil activation and deposition in end-organ tissue compartments causes collateral damage to parenchymal cells and thereby contributes largely to the development of (multiple) organ failure and ARDS/ALI. Experimental studies showed that trauma results in increased pulmonary neutrophil influx ${ }^{8-10,16}$. In addition to these quantitative investigations of the pulmonary neutrophil response to trauma, we characterized the pulmonary parenchymal neutrophil pool and provided a qualitative description of pulmonary neutrophil activation patterns. We thereby revealed that cell-surface receptor expression levels of Mac-1, LFA-1 and L-selectin on pulmonary neutrophils temporarily increased after trauma, and after that returned back to baseline levels within the first two weeks after the trauma. 

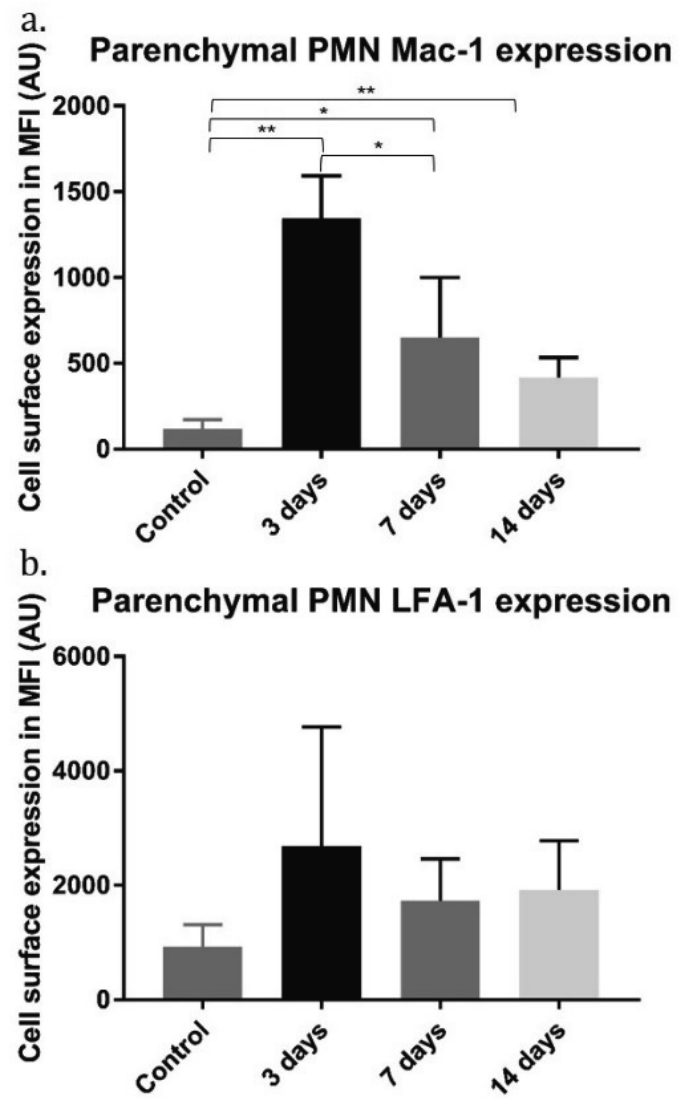

C.

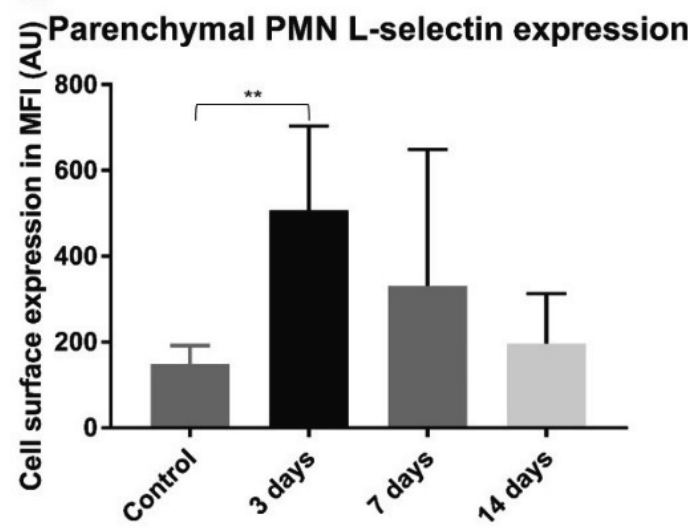

Figure 9.2. Changes in neutrophil cell surface expression of activation markers on parenchymal Neutrophils. Variations in cell surface expression levels of Mac-1 (a) and LFA-1 (b) and L-selectin (c). Data in mean fluorescence intensities; MFI in arbitrary units; AU. Significance between groups is displayed as, *, $p<0.05$; **, $p<0.01$. 


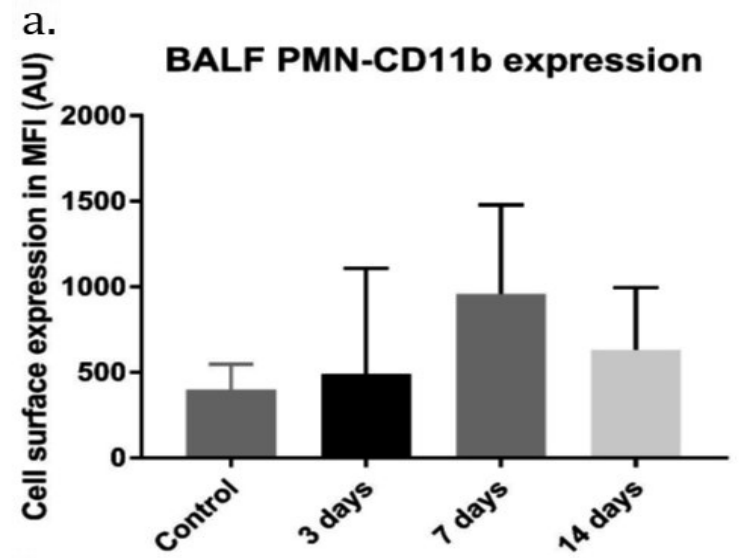

b.

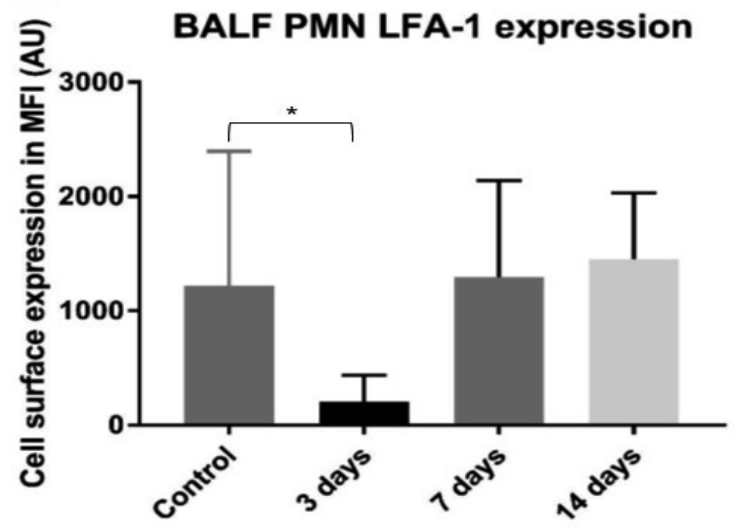

c.

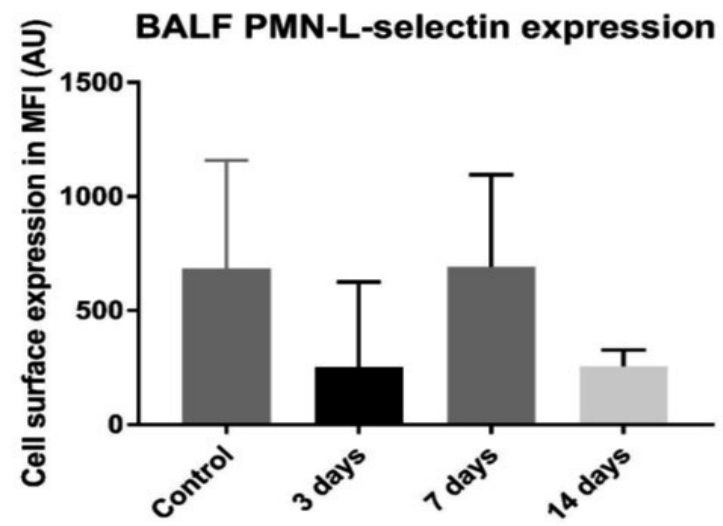

Figure 9.3. Changes in neutrophil cell surface expression of activation markers on Broncho-alveolar neutrophils. Variations in cell surface expression levels of Mac-1 (a) and LFA-1 (b) and L-selectin (c). Data in mean fluorescence intensities; MFI in arbitrary units; AU. Significance between groups is displayed as, * $p<0.05$. 
Neutrophil activation status is generally studied by comparing cell-surface expression levels of activation and migration markers. Relevant and validated markers include some that belong to the selectin and integrin receptor families ${ }^{25}$.
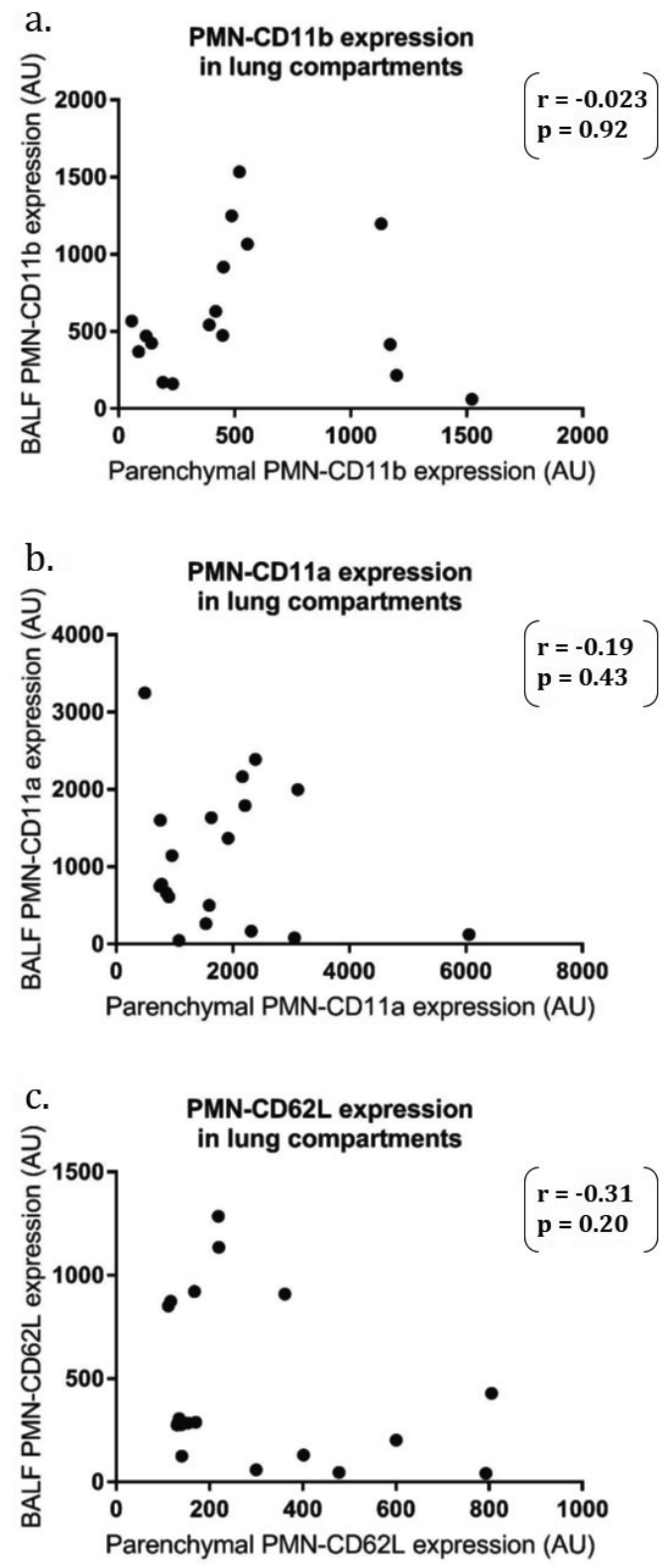

Figure 9.4. Correlation between parenchymal and broncho-alveolar neutrophil activation. 
Upon neutrophil activation, cells enter a primed state, and L-selectin expression decreases due to the shedding of the receptor from the cell membrane ${ }^{26}$. On the other hand, neutrophil Mac-1 expression increases in response to cell activation. These typical effects upon priming, however, have been identified and validated mainly in in vitro studies of blood neutrophils ${ }^{27,28}$. In line with the in vitro experiments on neutrophil activation, the current in vivo experiment also demonstrated a striking transient increase of Mac-1 cell-surface expression on parenchymal neutrophils after three days of observation. These findings are further in agreement with a previous trauma study by Van Wessem et al. in which hypovolemic shock caused an increase in CD11b expression on circulatory neutrophils ${ }^{29}$. Interestingly, in the current investigation, the expression of CD11b on BALF-neutrophils decreased simultaneously with the encountered increase of CD11b on parenchymal neutrophils. This finding may be suggestive of a specific biological function of the PMN-Mac-1 receptor in pulmonary neutrophil compartmentalization and PMN migration processes between the interstitium and the alveolar space. We also encountered increased neutrophil CD62Lexpression in the lungs upon activation. Increased pulmonary neutrophil-CD62L cellsurface expression levels can be explained either by decreased shedding of the receptor from the PMN membrane ${ }^{26}$ or by increased tissue infiltration of specific subtypes of CD62Lhigh neutrophils into the lung compartment ${ }^{30}$. In vivo studies on endotoxemia and trauma also demonstrated increased CD62L receptor expression on blood neutrophils ${ }^{31,32}$. We hypothesize that the encountered increase in total PMN-CD62L expression was partly caused by alterations in the constitution of the pulmonary neutrophil pool due to the appearance of novel (CD16low/CD62Lhigh) subsets in the circulation and subsequent tissue migration ${ }^{26,30-32}$.

Unfortunately, we were unable to identify and characterize this specific subset in our model, as PMN Fcy-receptor (CD16) expression cannot be investigated properly in rodent models due to relevant biological differences with the human Fcy-receptor family ${ }^{33,34}$. So, the increased CD62Lexpression on parenchymal neutrophils in our study might be explained by the increased selective pulmonary homing of this specific subset of neutrophils after trauma as well. LFA-1 is also of great importance in neutrophil tissue migration upon inflammation ${ }^{35}$. A trend towards higher parenchymal PMN-LFA-1-surface expression levels 72 hours after trauma was observed. Interestingly, a paradoxical effect was seen regarding LFA-1 expression on BALF neutrophils. This phenomenon suggests a potential specific biological role of the LFA receptor in neutrophil migration processes between the parenchymal compartment and the alveolar space.

Following initial altered pulmonary neutrophil activation within the first three days after trauma, activation markers return to homeostatic levels. The gradual normalization of activation status of both the parenchyma and the broncho-alveolar compartments is seen over time. This observed process of transient neutrophil activation and later restoration during the first weeks after trauma is in line with a clinical trauma study from Hietbrink et al. ${ }^{36}$.

Broncho-alveolar lavage is a clinically useful and relatively non-invasive method to study the inflammatory milieu of distal airways and alveoli. Most studies in the field of pulmonology, however, have been performed on lung biopsy samples. Variations exist between both sampling techniques regarding the constitution of samples in chronic inflammation ${ }^{37}$. In addition, we also found striking differences between both sampling 
techniques in the cases of trauma-induced acute systemic inflammation. Cell-surface expression of both L-selectin and integrins differ largely between the parenchymal neutrophil pool and the broncho-alveolar pool. These differences can be explained by potential biological functions of both receptors in neutrophil migration and compartmentalization processes $27,28,35$. As correlations between compartments were lacking in our standardized study, utilization of BALF to study the pulmonary compartment might not be an optimal method, and alternatives should be sought and further validated in upcoming studies.

For this study, an ice-cold PBS solution was used in a standardized fashion to collect BALF as this method is utilized in the clinical situation as well. Furthermore, it has been described that the addition of EDTA to the flush solution can cause the detachment of sequestered neutrophils 38 . Heparinized buffer solutions have the downside of potentially activating neutrophils due to in vitro-confirmed heparin-induced neutrophil activation ${ }^{39}$. Nevertheless, testing altered BALF-flushing solutions should be a focus of further research as this may improve the readout potential and representability of the BALF procedure for studying the pulmonary neutrophil pool.

This investigation has several limitations. Unfortunately, we were not able to investigate the functional capacities of different pulmonary neutrophil populations. It might be that the neutrophils residing in different compartments are in fact different phenotypes with specific functionality. Furthermore, it would be interesting to compare the formation potency of neutrophil extracellular traps (NETs) between populations 40 . Neutrophil extracellular traps are characterized by an extracellular filamentous chromatin-histone matrix enriched with neutrophil granular proteins such as myeloperoxidase and lactoferrin 41. They were demonstrated to facilitate PMN extracellular bacterial killing. Initially it was believed that neutrophil cell death was required to form NETs. However, a more recent study from Yousefi et al. demonstrated that under specific conditions, neutrophils were capable to form NETs out of mitochondrial-DNA without subsequent loss of cell viability. Interestingly, 'NETosis', the process of NET-formation, seems to be an important factor in the development of both acute and chronic inflammatory diseases ${ }^{42}$. The interplay between pulmonary neutrophil deposition, compartmentalization, activation, and NET-formation following orthopaedic trauma and intramedullary nailing should be a focus of upcoming studies.

\subsection{CONCLUSION}

In conclusion, the current study was the first to describe alterations in pulmonary neutrophil compartmentalization and activation following intramedullary nailing. We demonstrated that our standardized rat model is feasible to study the long-term innate immune response to trauma in all tissue compartments. As neutrophil cell-surface activation levels between parenchymal and bronchoalveolar neutrophil pools in our study were not correlated, BALF might not be an optimal readout to analyse characteristics of the heterogeneous pulmonary neutrophils. Therefore, it would be interesting to study alternative minimally invasive techniques for collecting representative lung neutrophils. Moreover, this standardized experiment revealed a specific pattern of transiently increased neutrophil deposition and increased PMN pulmonary activation. The encountered differences in integrin and selectin expression patterns between distinct pulmonary PMN pools imply a relevant biological role of 
integrins and L-selectin in migratory lung processes. These insights might be interesting targets for upcoming therapies aimed to intervene in pulmonary neutrophil infiltration and compartmentalization mechanisms in patients at risk for inflammatory complications after orthopaedic trauma.

\subsection{REFERENCES}

1. Pape HC, Auf'm'Kolk M, Paffrath T, Regel G, Sturm JA, Tscherne H. Primary intramedullary femur fixation in multiple trauma patients with associated lung contusion--a cause of posttraumatic ARDS? The Journal of trauma 1993; 34(4): 540-7; discussion 7-8.

2. Pape HC, Regel G, Dwenger A, Sturm JA, Tscherne H. Influence of thoracic trauma and primary femoral intramedullary nailing on the incidence of ARDS in multiple trauma patients. Injury 1993; 24 Suppl 3: S82103.

3. Dunn RH, Jackson T, Burlew CC, et al. Fat emboli syndrome and the orthopaedic trauma surgeon: lessons learned and clinical recommendations. International orthopaedics 2017; 41(9): 1729-34.

4. Carow J, Carow JB, Coburn M, et al. Mortality and cardiorespiratory complications in trochanteric femoral fractures: a ten year retrospective analysis. International orthopaedics 2017; 41(11): 2371-80.

5. Blankstein M, Byrick RJ, Nakane M, et al. Amplified inflammatory response to sequential hemorrhage, resuscitation, and pulmonary fat embolism: an animal study. The Journal of bone and joint surgery American volume 2010; 92(1): 149-61.

6. Adams JM, Hauser CJ, Livingston DH, Lavery RF, Fekete Z, Deitch EA. Early trauma polymorphonuclear neutrophil responses to chemokines are associated with development of sepsis, pneumonia, and organ failure. The Journal of trauma 2001; 51(3): 452-6; discussion 67.

7. Brown KA, Brain SD, Pearson JD, Edgeworth JD, Lewis SM, Treacher DF. Neutrophils in development of multiple organ failure in sepsis. Lancet 2006; 368(9530): 157-69.

8. Windsor AC, Mullen PG, Fowler AA, Sugerman HJ. Role of the neutrophil in adult respiratory distress syndrome. The British journal of surgery 1993; 80(1): 10-7.

9. Pape HC, Remmers D, Kleemann W, Goris JA, Regel G, Tscherne H. Posttraumatic multiple organ failure--a report on clinical and autopsy findings. Shock 1994; 2(3): 228-34.

10. Stormann P, Auner B, Schimunek L, et al. Leukotriene B4 indicates lung injury and on-going inflammatory changes after severe trauma in a porcine long-term model. Prostaglandins, leukotrienes, and essential fatty acids 2017; 127: 25-31.

11. Athens JW, Raab SO, Haab OP, et al. Leukokinetic studies. III. The distribution of granulocytes in the blood of normal subjects. The Journal of clinical investigation 1961; 40: 159-64.

12. Peters AM. Just how big is the pulmonary granulocyte pool? Clinical science 1998; 94(1): 7-19.

13. Hogg JC, Doerschuk CM. Leukocyte traffic in the lung. Annual review of physiology 1995; 57: 97-114.
14. Pillay J, Hietbrink F, Koenderman L, Leenen LP. The systemic inflammatory response induced by trauma is reflected by multiple phenotypes of blood neutrophils. Injury 2007; 38(12): 1365-72.

15. Relja B, Taraki R, Teuben MP, et al. Sera from severe trauma patients with pneumonia and without infectious complications have differential effects on neutrophil biology. BMC pulmonary medicine 2016; 16(1): 171.

16. Barletta KE, Cagnina RE, Wallace KL, Ramos SI, Mehrad B, Linden J. Leukocyte compartments in the mouse lung: distinguishing between marginated, interstitial, and alveolar cells in response to injury. Journal of immunological methods 2012; 375(1-2): 100 10.

17. Aulakh GK. Neutrophils in the lung: "the first responders". Cell and tissue research 2018; 371(3): 577 88.

18. Granton E, Kim JH, Podstawka J, Yipp BG. The Lung Microvasculature Is a Functional Immune Niche. Trends in immunology 2018; 39(11): 890-9.

19. Roger N, Xaubet A, Agusti C, et al. Role of bronchoalveolar lavage in the diagnosis of fat embolism syndrome. The European respiratory journal 1995; 8(8): 1275-80.

20. Osakabe Y, Takahashi Y. [Utility of the bronchoalveolar lavage procedure for the diagnosis of fat emboli syndrome (FES)]. Nihon Kokyuki Gakkai zasshi $=$ the journal of the Japanese Respiratory Society 1998; 36(11): 953-8.

21. Bonnarens F, Einhorn TA. Production of a standard closed fracture in laboratory animal bone. Journal of orthopaedic research : official publication of the Orthopaedic Research Society 1984; 2(1): 97-101.

22. Kobbe P, Vodovotz Y, Kaczorowski DJ, Mollen KP, Billiar TR, Pape HC. Patterns of cytokine release and evolution of remote organ dysfunction after bilateral femur fracture. Shock 2008; 30(1): 43-7.

23. Skrajnar S, Anzur Lasnik M, Bedina Zavec A. A flow cytometric method for determination of the blood neutrophil fraction in rats. Journal of the American Association for Laboratory Animal Science : JAALAS 2009; 48(2): 152-6.

24. Seekamp A, Jochum M, Ziegler M, van Griensven M, Martin M, Regel G. Cytokines and adhesion molecules in elective and accidental trauma-related ischemia/reperfusion. The Journal of trauma 1998; 44(5): 874-82.

25. Simon SI, Rochon YP, Lynam EB, Smith CW, Anderson DC, Sklar LA. Beta 2-integrin and L-selectin are obligatory receptors in neutrophil aggregation. Blood 1993; 82(4): 1097-106.

26. Ivetic A. A head-to-tail view of L-selectin and its impact on neutrophil behaviour. Cell and tissue research 2018; 371(3): 437-53. 
27. Neeley SP, Hamann KJ, White SR, Baranowski SL, Burch RA, Leff AR. Selective regulation of expression of surface adhesion molecules Mac-1, L-selectin, and VLA4 on human eosinophils and neutrophils. American journal of respiratory cell and molecular biology 1993; 8(6): 633-9.

28. Wagner JG, Roth RA. Neutrophil migration during endotoxemia. Journal of leukocyte biology 1999; 66(1): 10-24.

29. van Wessem KJ, Heeres $M$, Leliefeld $\mathrm{PH}$, Koenderman L, Leenen LP. Lipopolysaccharide and hemorrhagic shock cause systemic inflammation by different mechanisms. The journal of trauma and acute care surgery 2013; 74(1): 37-43; discussion -4.

30. Pillay J, Kamp VM, van Hoffen E, et al. A subset of neutrophils in human systemic inflammation inhibits T cell responses through Mac-1. The Journal of clinical investigation 2012; 122(1): 327-36.

31. Cocks RA, Chan TY, Rainer TH. Leukocyte L-selectin is up-regulated after mechanical trauma in adults. The Journal of trauma 1998; 45(1): 1-6.

32. Mommsen P, Barkhausen T, Hildebrand F, Zeckey C, Krettek C, van Griensven M. Regulation of L-selectin expression by trauma-relevant cytokines. Pathology, research and practice 2011; 207(3): 142-7.

33. Bruhns P. Properties of mouse and human IgG receptors and their contribution to disease models. Blood 2012; 119(24): 5640-9.

34. Farber DL, Sears DW. Rat CD16 is defined by a family of class III Fc gamma receptors requiring coexpression of heteroprotein subunits. Journal of immunology 1991; 146(12): 4352-61.
35. Lefort CT, Ley K. Neutrophil arrest by LFA-1 activation. Frontiers in immunology 2012; 3: 157.

36. Hietbrink F, Koenderman L, Althuizen M, Pillay J, Kamp V, Leenen LP. Kinetics of the innate immune response after trauma: implications for the development of late onset sepsis. Shock 2013; 40(1): 217.

37. Anderson BO, Brown JM, Harken AH. Mechanisms of neutrophil-mediated tissue injury. The Journal of surgical research 1991; 51(2): 170-9.

38. Olivier CE dSR, Lima RPS, Argentao DGP, da Silva GKM, da Silva MD A novel utility for an old method: The leukocyte adherence inhibition test is an easy way to detect the immunoreactive interference of the collection tube anticoagulant on cellular immunoassays. . J Cell Adhesion 2014; 2014: 1 /6.

39. El Habbal MH, Smith L, Elliott MJ, Strobel S. Effect of heparin anticoagulation on neutrophil adhesion molecules and release of IL8: C3 is not essential. Cardiovascular research 1995; 30(5): 676-81.

40. Joshi MB, Lad A, Bharath Prasad AS, Balakrishnan A, Ramachandra L, Satyamoorthy K. High glucose modulates IL-6 mediated immune homeostasis through impeding neutrophil extracellular trap formation. FEBS letters 2013; 587(14): 2241-6.

41. McIlroy DJ, Jarnicki AG, Au GG, et al. Mitochondrial DNA neutrophil extracellular traps are formed after trauma and subsequent surgery. Journal of critical care 2014; 29(6): 1133 e1-5.

42. Yousefi S, Simon HU. NETosis - Does It Really Represent Nature's "Suicide Bomber"? Frontiers in immunology 2016; 7: 328 
General discussion \& conclusions of this thesis

Martijn Hofman 


\subsection{PART I: INFLUENCE OF TBI ON FRACTURE HEALING}

TBI is recognised to induce both central and systemic changes, a number of which have the potential to affect bone and bone fracture healing. Clinical observations as well as expert opinions claim enhanced bone healing in patients with concomitant severe TBI, although the relationship between TBI and fracture healing is still poorly understood.

Therefore, we aimed in part I of this thesis to find answers to the question of whether concomitant TBI definitely enhances fracture healing. And if so, what pathophysiological mechanisms are underlying this effect?

The first review on this topic by Morley et al. ${ }^{1}$ could not answer the question of whether there is positive interaction between TBI and fracture healing. More recently, in our review described in chapter 2 , we observed a consensus of the scientific community over the positive influence of TBI on fracture healing, although the underlying pathophysiologic mechanisms of enhanced callus formation in patients with concomitant TBI remain unknown. Since the publication of our review in 2015, several new experimental as well as clinical studies have been published on this topic.

Experimental studies. In the experimental research setting, several studies have been published after our review, which provide further evidence for the positive influence of TBI on fracture healing. These studies demonstrate the enhanced callus formation and superior mechanical integrity of the fracture site in murine TBI/fracture models ${ }^{2-4}$. Several researchers have hypothesised that the enhanced callus volumes post-TBI may represent a form of heterotopic ossification (HO); however, no gross morphological differences exist in the callus structure between the patients with and without concomitant TBI. Localised inflammation in combination with neurological injury is suggested to drive this abnormal bone formation ${ }^{5}$. Although the callus formation at $\mathrm{HO}$ and fracture sites may be equal in composition and quality, the soft-tissue environment of HO, often peri-articular, may not be as favourable for bone formation as that of fracture sites.

Moreover, studies have suggested that the central and peripheral nervous system plays a significant role in regulating bone homeostasis and that an injury-induced disturbance of neural pathways alters the bone regeneration response during fracture healing. According to this theory, several studies have shown a positive correlation between increased leptin concentrations after TBI and enhanced callus formation and bone healing 6-8. After TBI, the expression of leptin in the brain is upregulated and the leptin concentrations in both cerebrospinal fluid (CSF) and serum are increased, leading to faster callus formation ${ }^{6}$. In leptin-deficient mice, the research group of Graef and Seemann showed decreased callus formation, impaired bony bridging of fractures and high non-union rates compared with wild type mice 7,8 .

Other studies ${ }^{9-11}$ have demonstrated that the serum from brain-injured rats increased the proliferation of mesenchymal stem cells and that the serum and CSF from patients with TBI increased osteoblastic proliferation, indicating the role of humoral mechanisms.

Building up on the observation in our review that many studies focus on the role of mesenchymal stem cells (MSCs), Liu et al. studied the influence of the stromal cellderived factor-1/CXC-Motiv-Chemokine receptor 4 (SDF-1/CXCR4) axis and its role in the 'homing' of MSCs at the fracture site during bone repair in a murine fracture model 12. In post-TBI mice, an upregulation of the SDF-1/CXCR4 axis after TBI and enhanced 
callus formation were shown. Inhibition of SDF-1 resulted in the decrease of callus formation again. Therefore, they concluded that SDF-1, as a chemoattractant for MSCs during bone regeneration, is a factor that seems to play a pivotal role in the enhanced fracture healing after TBI.

Clinical studies. In the clinical studies published after our review, the influence of neuroendocrine pathways on bone regenerations comes to the fore. The assumption that TBI has a substantial impact on skeletal regeneration and remodelling is reasonably based on the central role of the hypothalamic nuclei in managing neuroendocrine humoral outflow via the pituitary gland. The tissue damage after a TBI results in the release of tissue debris, prostaglandins and reactive oxygen and nitrogen elements, which in turn cause a response of resident immune cells, an upregulation of leukocyte migration and recruitment and the release of inflammatory mediators, such as cytokines and chemokines. This systemic and local immune response is accompanied by a neurogenic inflammation process in which different neuro-humors, neurotransmitters or neuro-hormones with osteogenic characteristics are systemically and locally released at the fracture site, thus playing a crucial role in bone regeneration 13-16.

This vision was supported by a prospective controlled study performed by Khallaf et al., who showed an accelerated fracture healing process in patients with concomitant TBI based on the involved neuro-hormonal mechanisms ${ }^{17}$. The healing time was shorter, the callus volume was larger and the concentrations of parathyroid and growth hormone were elevated in this group of patients; moreover, the (nor-)adrenaline level was lower, reflecting a relative inhibition of nerve signalling of the sympathetic nervous system via neuromediators in the hypothalamus after TBI. This in turn could lead to the substantial mobilisation of undifferentiated mesenchymal stem cells and osteoprogenitor cells to the peripheral circulation to induce accelerated abundant healing of long-bone fractures, indicating the combined neuro-hormonal mechanism that explains this accelerated healing. The normal level of corticosteroids in patients with severe head injury and associated long-bone fractures could reflect a relative neuronal inhibition of the suprarenal cortex in producing anti-osteogenic corticosteroids, thus inducing an inflammatory condition, which is mandatory to bone healing 17.

Wang et al. also showed enhanced osteogenesis in patients with a combination of fractures and spinal cord injury - namely, increased callus volumes and shorter healing times. However, in this study, all patients were classified as Frankel grade A, meaning they had no motor or sensory function, which could be a reason for the reduced mobilisation and subsequent callus formation in these patients ${ }^{18}$.

The research groups of both Zhuang and Li and Zhang et al. found evidence for the role of nerve growth factor (NGF) in the acceleration of fracture healing 19,20. This polypeptide hormone plays an important role in the survival of nerve centres and peripheral neurons by adjusting their growth, development and differentiation ${ }^{21}$. The concentration of NGF was demonstrated to significantly increase after injury in patients with limb fractures and TBI compared with the patients with only limb fractures. The proposed mechanism of action was that NGF increases the nerve ingrowth into the callus, stimulates the local angiogenesis and thereby promotes bone regeneration. Supportive to this theory, Zhuang and Li demonstrated an increased concentration of 
epidermal growth factor, which simultaneously acts on vascular endothelial cells, fibroblasts and osteoblasts to promote the formation of bone tissue and to accelerate the synthesis and deposition of the matrix ${ }^{19}$. Furthermore, Zhang et al. demonstrated an increased level of vascular endothelial growth factor (VEGF), which mediates local angiogenesis at the fracture site ${ }^{20}$. Zhang et al. also demonstrated that the percentages of M2 macrophages significantly increased during fracture healing, especially in patients with concomitant TBI who showed larger callus volumes and shorter healing times. These M2 macrophages are recruited to the fracture site in the later inflammatory stage of fracture healing, and they secrete tissue repair factors such as IL10 , TGF- $\beta$, BMP 2 and VEGF to initiate the anti-inflammatory response; moreover, they attract mesenchymal stem cells and induce osteochondral differentiation and angiogenesis 22 .

In summary, there are strong indications, both experimental and clinical, for an influence of TBI on fracture healing via different neuro-humoral and neuro-hormonal pathways. Because the above described underlying pathomechanisms are far from uniform, these provide us with multiple leverage points to base future studies on. In our retrospective clinical study described in chapter 3, we demonstrated that the fracture stabilisation strategy has more impact on fracture healing and non-union development than concomitant injuries, such as TBI and chest injury. Moreover, we did not identify TBI as an independent factor for non-union development. Furthermore, the clinical studies supporting the expert opinion that TBI positively influences fracture healing are ambiguous and have low comparability and extent of the patient population. Therefore, it would be of immense interest to set up a large prospective multicentre study on patients with long-bone fractures and concomitant TBI to clinically endorse or reject the hypothesis that TBI positively influences fracture healing.

The results of part I of this thesis emphasise the importance and potential of further clinical as well as experimental research on the topic of fracture healing and its pathomechanisms.

Overall, over the last decades, evidence for the relationship between neural and humoral pathways and fracture healing has been increasingly provided in experimental studies, but the exact pathophysiological mechanisms are not yet clear. In part II of this thesis, we further elaborated on some of these issues; however, because the pathophysiological complex of fracture healing contains multiple influencing factors, which are also intertwined with each other, there will be much to be gained in this field of research in the next years. Further research will provide knowledge on pathophysiologic mechanisms, which can eventually be used to diagnose patients at risk for non-unions or delayed unions and to alter their treatment strategy.

\subsection{PART II: NEURO-HUMORAL MODULATION OF FRACTURE HEALING}

Attempts to modulate the inflammatory response of the body in stress situations, such as organ dysfunction or sepsis, to improve survival are often unsuccessfully undertaken. The innate immune system appears to be barely responsive to the immunosuppressive drugs used in current treatment strategies. Besides the evidence that the use of immunosuppressive agents is counterproductive for bone healing, the modulation of fracture healing remains difficult. Part of the aims of this thesis was to expound some leverage points in the normal neuro-humoral modulation mechanism of 
bone regeneration, which could possibly influence the fracture healing process. In part II of this thesis, these aspects are studied and described.

As mentioned before, a significant role in regulating bone homeostasis and fracture healing is also imputed to the central and peripheral nervous system and to injuryinduced disturbances of neural pathways.

On this basis, in chapter 4, we studied the influence of the blockage of substance $\mathrm{P}, \mathrm{a}$ neurotransmitter released by the sensory nerve-endings in bone tissue, on fracture healing.

We demonstrated that this blockage impaired not only the expression of osteogenic genes but also the quality of the callus formed and the biomechanical strength in fracture healing. These results, together with the results of previous studies on this topic by Offley et al. ${ }^{23}$ and Apel et al. ${ }^{24}$, provide strong indications for the connection between neural pathways and bone regeneration.

Although these results may not have direct consequences for the current treatment of patients with fractures or disturbed fracture healing, they provide a sound basis for further research on the influence of substance $\mathrm{P}$ on fracture healing. A next step in research could be to study the influence of the local or systemic administration of substance P on fracture healing in small animal fracture models. Another possibility, with the results of our study in mind, is a study in which the influence of substance $P$ blockage on fracture healing is investigated in a combined small animal fracture and TBI model. Apel et al. have already shown that the blockage of both neurotransmitters (i.e. substance $\mathrm{P}$ and CGRP) from sensory nerves results in impaired fracture healing; therefore, further research on the role of CGRP in fracture healing is also promising. The results of such studies could eventually lead to the use of specific neurotransmitters as diagnostic markers or therapeutic agents in clinical situations.

For the clinical outcome of patients with lower extremity fractures, in addition to the biomechanical strength of the healed bone, the quality of the mobility of patients is very important and significantly determines the quality of life after fracture healing. Therefore, being capable to perform an adequate evaluation of the gait of patients is crucial in the clinical setting as well as in the experimental setting of fracture research. Therefore, we wanted to implement a method for gait analysis in small animal fracture models because these models constitute about $50 \%$ of experimental fracture healing research.

In chapter 5, we evaluated - to the best of our knowledge - for the first time the CatWalk gait analysis system for gait analyses in a femur fracture rat model. Our results showed that the CatWalk system is suitable for gait analysis in small animal fracture models and that the evaluated gait patterns have high correlation with the osteogenesis of fracture healing in these models. Because clinical interest in gait analyses is increasing and gait disturbances have a significant impact on the quality of life of patients with lower extremity fractures, we presume that the use of this gait analysis system in fracture healing research meets a need and the expectations of researchers in this field. Moreover, we suppose that the CatWalk system will be preferred above other methods, such as treadmill measurements, because in this system, the ranges of both static and dynamic parameters, which can be evaluated at the same time, are more comprehensive. For instance, using this system, the pressure exerted by each paw, the 
swing and stand phase of each leg and the percentage of the time spent bearing weight in each walk cycle for the given paw can be evaluated. Furthermore, these data can be simultaneously compared with the healthy side. We therefore expect the CatWalk gait analysis system to be increasingly used in small animal fracture research, which we strongly advocate because it can provide a considerable amount of data and knowledge for gait analysis development after lower extremity fractures.

To date, the most important tools for diagnosing disturbed fracture healing are clinical and radiological findings. Although research on the possible biomarkers that can be used as predictors for non-union development is promising, achieving consensus is very difficult because the evidence available is heterogeneous ${ }^{25}$. Moreover, when a non-union is diagnosed, the exact treatment and the time point of this non-union treatment are still under discussion. Because the socio-economic impact of disturbed fracture healing is immense, finding biomarkers, which can predict the prognosis of the fracture healing process and the outcome of certain procedures performed in cases of non-unions, would be very valuable.

The results of our study described in chapter 6 most importantly indicate a significantly lower ornithine concentration and arginase- 1 expression in the bone marrow of patients developing non-unions. Moreover, this was the first study to recognise the possible biomarkers that could be used as predictors of the outcome of the autologous bone grafting procedure by reamer-irrigator-aspirator (RIA) in cases of non-union. These possible biomarkers include arginine, ornithine and iNOS.

Therefore, this study could be a starting point to further investigate these biomarkers in the fracture healing process and to determine certain cut-off points for the different biomarkers, based on which the prognosis of fracture healing and non-union treatment could be estimated.

The concentrations and expressions of these supposed 'biomarkers' give an indication on the prognosis of the non-union treatment in the case when the non-union has already occurred. Knowing the level of these concentrations and expressions at the time of the initial fracture would be more interesting so as to be able to say something on non-union development.

In multivariate analysis, iNOS was the only significant factor within the included variables (i.e. age, BMI, NUSS, arginine-, citrulline- and ornithine concentrations and arginase-1 and iNOS expression). Differences in the molecular patterns in bone grafts between patients with success and failure at a site distant from the non-union may indicate that systemic molecular pathologies are partly responsible for the failure of non-union treatment and that the non-union is not a purely local metabolic problem. Previous studies have shown that the callus tissue and plasma samples of patients with hypertrophic and atrophic non-unions have abnormal low concentrations of amino acids arginine, citrulline and ornithine when compared with those of patients with normal healed and acute fractures ${ }^{26}$.

Increased iNOS expression suggests a prolonged inflammatory response (i.e. stimulation by NF- $\kappa \mathrm{B}$ ) that results in the production of pro-inflammatory cytokines such as IL-1, TNF- $\alpha$ and IFN- $\gamma$. A disturbed chronic inflammatory response during fracture healing might result in delayed union or non-union development and this could be a reason that the reaction of the bone tissue to the RIA treatment is inadequate 27,28 . 
The significantly lower arginine concentrations that coincide with the higher iNOS expression in patients with refractory non-unions may indicate the depletion of this amino acid by an increased catabolic response of the patient ${ }^{29}$.

Arg1 is the enzyme that converts arginine into ornithine, which can subsequently lead to collagen synthesis. RIA procedures resulting in successful non-union healing as well as in unsuccessful healing showed a 3-4-fold lower arginase-1 expression than normal healed fractures. This might reflect the inadequate anabolic response of the bone during the healing process, which initially was the cause of the non-union development and the need for surgical repair. This is also reflected by the lower ornithine concentrations measured in the reamed intramedullary aspirate in these patients.

Although the NUSS score is a known factor in patients with a fracture that defines the risk of subsequent non-union development, this study found the NUSS score to be a predictor of the success rate of the non-union treatment. In addition, compared with the NUSS score, the activity of the inflammatory response in the grafted material obtained by RIA was a better predictor of therapy success.

A limitation of this study is the heterogeneity of different patient characteristics in a relatively small patient population. Ideally, a large cohort of patients with similar characteristics in all groups is needed to minimise the possible confounding effects; therefore, this study should be regarded as a hypothesis generating pilot study.

Owing to the diversity of the cell types involved in the complex process of fracture healing, synergistic interactions between these cells are essential; moreover, it is reasonable and proven that micro-vesicles (MVs) play an important role in this intercellular communication ${ }^{30-33}$. However, the knowledge on the role of MVs and especially of systemically derived MVs in fracture healing is still sparse.

In chapter 7, we demonstrated that systemically released MVs isolated after a femoral fracture were time-dependently incorporated in osteoblasts and concentrated around their nucleus. Further, we showed that these MVs modulated the viability but not the differentiation of osteoblasts after trauma.

Although we do not detail in our study the exact pathophysiological mechanisms of action or the content of these MVs, it is known from literature that these MVs are active in many physiological and pathophysiological processes in the human body and that they play an important role in intercellular communication during fracture healing, between cells locally or at a distance through receptor-mediated interactions or by delivering their protein, lipid and genetic contents ${ }^{34-38}$.

The role of MVs and related vesicles (i.e. exosomes), which can be generated by various cell types, has gained interest over the last years. Different studies have demonstrated that exosomes have the potential to induce regenerative processes during bone formation, accomplished by activating the native cells (especially mesenchymal stem cells), upregulating growth factor genes, regulating osteogenesis, enhancing angiogenesis and increasing the expression levels of specific proteins involved in matrix mineralisation ${ }^{33,39-42}$.

In the literature, the important role of paracrine signalling of MSCs that differentiate to regenerate bone defects is emphasised ${ }^{43-45}$. Therefore, the proliferating and migrating capacity of MSCs is crucial for fracture healing. Osteoblasts, as a differentiated product 
from MSCs, are important for the mineralisation process of callus because they produce calcium- and phosphate-based minerals ${ }^{46}$. The pivoting role of the regulation of osteoblast proliferation and osteogenic differentiation in bone regeneration is supported by the results of our study presented in chapter 8 of this thesis.

Micro-vesicles incorporate various components from the originating cells, including lipids, proteins, mRNA, miRNA and other components $38,47,48$; in particular, miRNAs are supposed to have a central role in bone regeneration. In the review by Hao et al., the upregulation of certain miRNAs in exosomes and their modulatory effect on the target genes and pathways in the regulation of osteogenic differentiation is substantiated by the studies of multiple authors ${ }^{39}$.

In another possible pathway, mentioned in the review by Hao et al., other contents of the vesicles, such as cytokines [monocyte chemoattractant protein 1 (MCP-1) and stromal cell-derived factor 1 (SDF-1)], activate or are delivered to the target cells at the fracture site as mode of action of the MVs ${ }^{39}$.

In short, the pathway of MVs from resident cell to target cell and the exact consequences of the release of their content at the fracture site are very complex and regulated by multiple factors, which are still to be elucidated by future research.

The biological characteristics and structure of exosomes and MVs make them appropriate to act as a diagnostic or therapeutic tool in cases of fracture healing or delayed fracture healing. Encapsulation by the lipid bilayer of the exosomal membrane protects the proteins and miRNAs of exosomes from degradation in the body fluid, contributing to their ability to deliver the content across the cell membrane into the cytosol of the recipient cells 49,50. Compared with biomaterial treatment, the composition of these vesicles resolves the problems of immunogenicity and toxicity. In addition, exosomes are considerably stable and can be preserved for approximately six months in vitro at $-20{ }^{\circ} \mathrm{C}$ without loss of potency ${ }^{51}$. Nevertheless, the process from formation to effect is fairly complex and is regulated by multiple factors. The related mechanisms are still at an early stage of comprehension and require further investigations ${ }^{48,52}$.

In addition, the exact mechanisms of the enhanced bone formation after exosome treatment remain elusive. Thus, exosomes from different sources may exhibit diverse effects on fracture healing.

Future research therefore needs to investigate the characteristics of the process in which MVs play the key role in influencing fracture healing, including the origin and composition of the MVs, the mechanism of action of different constituents within the MVs, the exact target cells of the MVs, etc.

If these questions are answered, the way in which the responsible contents of MVs can be used to help in the diagnostics or treatment of fractures should be addressed. Possible applications could be the adjustment of the content or local application of MVs to alter the fracture healing process. Because the current exosome isolation methods such as ultracentrifugation and ultrafiltration only provide a low exosome yield ${ }^{53}$, the challenge of obtaining sufficient amounts of exosomes has to be overcome ${ }^{39}$.

In the field of cellular immune response to trauma, neutrophils play an important role. In addition to their role as eliminator of detrimental stimuli, they modulate the function of multiple components of the innate and adaptive immune system. The functionality of neutrophils is reflected by the cell-surface expression of selectins and integrins. 
In the study described in chapter 8, we demonstrated the changes in the circulatory neutrophil population in a standardised long-term observation fracture model, primarily focusing on the later inflammatory phase ( $>48 \mathrm{~h}$ after trauma) rather than the early/instant immune response. A temporary decrease in the number and activation status of the circulatory neutrophils was shown three days after femoral fracture and intramedullary nailing. The decreased activation was characterised by a reduced $\alpha_{M} \beta_{2}$-integrin (MAC-1 or CD11b/CD18) and an increased $\alpha_{\mathrm{L}} \beta_{2}$-integrin (LFA-1 or CD11a/CD18) expression. The transient decrease in neutrophil numbers could be caused by the homing of neutrophils into the fracture hematoma, regulated by the release of mitochondrial damage-associated molecular patterns (DAMPs) and the subsequent release of different chemoattractants. This homing of neutrophils into the fracture haematoma is in line with the results of several experimental and clinical studies that describe this phenomenon after fracture ${ }^{54-58}$.

Other important features of the inflammatory response after fracture are the extent, duration and sufficient termination of this response because enhanced migration of neutrophils into other organ systems after long-bone fractures and intramedullary stabilisation has also been described and could justify our finding of decreased circulatory neutrophil numbers. In this context, the lungs have been identified as a primary target organ, in which neutrophils potentially damage parenchymal lung tissue ${ }^{59}$ and cause complications such as acute lung injury and ARDS 60-62. Moreover, in the case of insufficient termination of the immune response, excessive homing of neutrophils into the fracture haematoma can occur and this could be damaging to the fracture healing process as well ${ }^{63-65}$.

The total range of functions of neutrophils as activators and regulators of different cells and processes within the non-specific as well as specific immune response is still to discover.

The functionality of neutrophils is regulated by the neutrophil surface receptor expression, which can detect different chemoattractants and define different neutrophil subsets or phenotypes. We examined the expression of selectins and integrins; moreover, we demonstrated an increase in heterogeneity and thereby a change in the functionality of the circulatory neutrophil pool during the restoration of neutrophil homeostasis after fracture.

We proved that a new subset of unique CD11 $\mathrm{b}^{\text {high }} / \mathrm{CD} 11 \mathrm{a}^{\text {high }}$ neutrophils was present in the post-inflammatory neutrophil pool. This demonstrates a post-traumatic cell surface receptor dynamic in circulating neutrophils and an increased heterogeneity of the blood neutrophil pool during the restoration phase after trauma. Owing to the wide range of functions of neutrophils, it is reasonable that phenotypic and functional diversity exists within the neutrophil pool and that subpopulations of neutrophils are identified 66 . Because the neutrophils of this CD11 $\mathrm{b}^{\text {high }} / \mathrm{CD} 11 \mathrm{a}^{\text {high }}$ subset have a significantly higher co-expression with very late antigen-4 (VLA-4/CD49d) than regular neutrophils, as we have shown, they most likely represent a unique phenotype.

The expression of CD49d has been identified on neutrophil progenitor cells in the bone marrow; therefore, it is tempting to hypothesise that these CD $11 \mathrm{~b}^{\text {high }} / \mathrm{CD} 11 \mathrm{a}^{\text {high }}$ neutrophils are released from the bone marrow 67,68 . 
On the contrary, whether these neutrophils with distinct functions belong to separate developing lineages or embody certain activation states of a common precursor is still not proved ${ }^{69}$. In the last years, increasing attention is being paid to neutrophil heterogeneity 70 because this could be very interesting for future selective interventions. Certain therapies applicable to all neutrophils are mostly accompanied by the aggravating complications of neutropenia; therefore, the possibility to modulate specific subsets of neutrophils opens a wide range of possibilities in all kinds of treatments. Moreover, in fracture healing, the presence of different phenotypes of neutrophils, such as those demonstrated in our study, could offer opportunities in the treatment of disturbed fracture healing. Because our study was the first to describe these changes in the blood neutrophil pool in a standardised femoral fracture model, it can form the basis for the further research of novel immunotherapeutic strategies to modulate neutrophil homeostasis after fractures.

Besides the influence of an intramedullary treated femoral fracture on the circulating neutrophils, in chapter 9, we studied the explicit influence of this pathology on the pulmonary neutrophil pool in rats. In line with the decreased circulating neutrophil numbers and activation three days after fracture induction, we found an increased pulmonary neutrophil deposition as well as a specific neutrophil activation pattern, characterised by increased selectin and integrin receptor expression in this period of fracture healing.

As described by Deniset et al., the lungs, besides the skin and the spleen, function as a reservoir for neutrophils ${ }^{70}$. One theory on the function of these pulmonary neutrophils is that they reside in the pulmonary capillaries and perform a kind of immune surveillance ${ }^{71}$. Another theory is that older neutrophils eventually return to the lungs to de-prime and express novel homing receptors for migration to the bone marrow to undergo apoptosis ${ }^{72}$. A third theory is that the intrapulmonary neutrophils form an original, different subset with a distinct function ${ }^{73}$.

Although a novel subset of neutrophils within the pulmonary tissue is not verified until now, the results of our study on the pulmonary neutrophil pool in chapter 9 show a certain compartmentalisation related to intrapulmonary neutrophil heterogeneity, with impressive differences between the neutrophils belonging to the pulmonary parenchymal and broncho-alveolar compartments. This could indicate a limited readout potential of broncho-alveolar lavage fluid (BALF) analysis to evaluate the pulmonary neutrophil pool.

We further demonstrated a substantial increase of the cell-surface expression of both the integrin MAC-1 and the selectin CD62L on pulmonary neutrophils after an isolated femur fracture. These findings might be explained by the increased selective pulmonary homing of a specific subset of neutrophils after trauma.

These phenomena could also increase the possibility of neutrophil NET production leading to tissue damage within the lungs, thus causing inappropriate inflammatory situations such as ARDS in poly-traumatised patients 69 . Overall, future research to dispose the identity of neutrophil subpopulation or phenotypes should be propagated because more questions about the activities, functions and roles of neutrophils in fracture healing continuously originate. 


\subsection{CONCLUSIONS OF THIS THESIS}

As demonstrated throughout this thesis, fracture healing is still a very complex, kaleidoscopic process, many aspects of which are still not clear. The process has multiple influencing factors, which relate and influence each other and sometimes have opposing effects depending on the phase in which the fracture healing process is situated.

In chapter $\mathbf{2}$ and 3, we demonstrated that the evidence for the positive influence of TBI on fracture healing and bone regeneration is increasing, especially in experimental studies. On the contrary, prospective clinical trials to support these experimental data are still missing.

In chapter 4, we showed the negative effects of neurokinin-1-receptor blockage on the expression of different osteogenic proteins and on the biomechanical strength of bone healing. This means that substance $\mathrm{P}$ is important for a normal bone healing process and that this neurotransmitter offers great potential for future research related to fracture healing.

In chapter 5, we successfully introduced the CatWalk system for gait analysis in fracture healing studies in small animal models. The gait analysis with this system is accurate, and the system provides clinically relevant and extensive static as well as dynamic data on gait patterns in a non-invasive, longitudinal manner during fracture healing in small animal models. The CatWalk system has the potential to become a standard gait analysis method in fracture healing research in experimental small animal models, thereby improving knowledge about behavioural and locomotor recovery after lower extremity fractures.

In chapter 6, we investigated the influence of amino acid metabolism in fracture healing and found that the arginine-NO metabolism in the bone marrow influences the outcome of non-union treatment, with indications for a prolonged inflammatory response in patients with unsuccessful bone grafting therapy. The determined arginine concentrations and Nos2 expression could be used as predictors for the successful treatment of autologous bone grafting in non-union treatment.

In chapter 7, we demonstrated that MVs from trauma plasma increased the viability but did not stimulate the differentiation of osteoblasts, particularly in the late phase of fracture healing. These acknowledgements, together with the growing scientific interest in exosomes, MVs and apoptotic bodies, will contribute to a better understanding of the intercellular communication processes in fracture healing.

In chapter 8 and 9, we showed that in the fracture healing process, a transient decrease in circulating neutrophil numbers and a transient increase in the pulmonary deposition of neutrophils with a specific activation pattern occur. The latter phenomenon might play an important role in the pathomechanism of ARDS after trauma. Furthermore, we demonstrated the increased heterogeneity of the blood neutrophil pool during the restoration phase of neutrophil homeostasis by identifying a novel subset of CD11 $b^{\text {high }} / \mathrm{CD} 11 \mathrm{a}^{\text {high }}$ neutrophils appearing one week after the intramedullary nailing of a femur fracture.

Finally, in this thesis, we contributed towards the further understanding of a few aspects of neuro-humoral modulation of the fracture healing process, although we may 
have launched more questions than provided answers within this difficult but exciting subject matter. Therefore, this thesis forms an excellent basis for future research in this field, which will improve our understanding of fracture healing processes and their influencing factors.

\subsection{REFERENCES}

1. Morley J, Marsh S, Drakoulakis E, Pape HC, Giannoudis PV. Does traumatic brain injury result in accelerated fracture healing? Injury 2005; 36(3): 363-8.

2. Brady RD, Grills BL, Church JE, et al. Closed head experimental traumatic brain injury increases size and bone volume of callus in mice with concomitant tibial fracture. Scientific reports 2016; 6: 34491.

3. Tsitsilonis S, Seemann R, Misch M, et al. The effect of traumatic brain injury on bone healing: an experimental study in a novel in vivo animal model. Injury 2015; 46(4): 661-5.

4. Locher RJ, Lunnemann T, Garbe A, et al. Traumatic brain injury and bone healing: radiographic and biomechanical analyses of bone formation and stability in a combined murine trauma model. Journal of musculoskeletal \& neuronal interactions 2015; 15(4): 309-15.

5. Genet F, Kulina I, Vaquette C, et al. Neurological heterotopic ossification following spinal cord injury is triggered by macrophage-mediated inflammation in muscle. The Journal of pathology 2015; 236(2): 229-40.

6. Yan H, Zhang HW, Fu P, et al. Leptin's effect on accelerated fracture healing after traumatic brain injury. Neurological research 2013; 35(5): 537-44.

7. Graef F, Seemann R, Garbe A, et al. Impaired fracture healing with high non-union rates remains irreversible after traumatic brain injury in leptindeficient mice. Journal of musculoskeletal \& neuronal interactions 2017; 17(2): 78-85.

8. Seemann R, Graef F, Garbe A, et al. Leptin-deficiency eradicates the positive effect of traumatic brain injury on bone healing: histological analyses in a combined trauma mouse model. Journal of musculoskeletal \& neuronal interactions 2018; 18(1): 32-41.

9. Boes M, Kain M, Kakar S, et al. Osteogenic effects of traumatic brain injury on experimental fracture-healing. The Journal of bone and joint surgery American volume 2006; 88(4): 738-43.

10. Gautschi OP, Toffoli AM, Joesbury KA, Skirving AP, Filgueira L, Zellweger R. Osteoinductive effect of cerebrospinal fluid from brain-injured patients. Journal of neurotrauma 2007; 24(1): 154-62.

11. Bidner SM, Rubins IM, Desjardins JV, Zukor DJ, Goltzman D. Evidence for a humoral mechanism for enhanced osteogenesis after head injury. The Journal of bone and joint surgery American volume 1990; 72(8): 1144-9.

12. Liu X, Zhou C, Li Y, et al. SDF-1 promotes endochondral bone repair during fracture healing at the traumatic brain injury condition. PloS one 2013; 8(1): e54077.

13. Bajwa NM, Kesavan C, Mohan S. Long-term Consequences of Traumatic Brain Injury in Bone Metabolism. Frontiers in neurology 2018; 9: 115.
14. Ziebell JM, Morganti-Kossmann MC. Involvement of pro- and anti-inflammatory cytokines and chemokines in the pathophysiology of traumatic brain injury. Neurotherapeutics : the journal of the American Society for Experimental NeuroTherapeutics 2010; 7(1): 22-30.

15. Lu J, Goh SJ, Tng PY, Deng YY, Ling EA, Moochhala S. Systemic inflammatory response following acute traumatic brain injury. Frontiers in bioscience 2009; 14: 3795-813.

16. Sorby-Adams AJ, Marcoionni AM, Dempsey ER, Woenig JA, Turner RJ. The Role of Neurogenic Inflammation in Blood-Brain Barrier Disruption and Development of Cerebral Oedema Following Acute Central Nervous System (CNS) Injury. International journal of molecular sciences $2017 ; \mathbf{1 8}(8)$.

17. Khallaf FG, Kehinde EO, Hussein S. Bone Healing and Hormonal Bioassay in Patients with Long-Bone Fractures and Concomitant Head Injury. Medical principles and practice : international journal of the Kuwait University, Health Science Centre 2016; 25(4): 336- 42 .

18. Wang L, Yao X, Xiao L, et al. The effects of spinal cord injury on bone healing in patients with femoral fractures. The journal of spinal cord medicine 2014; 37(4): 414-9.

19. Zhuang YF, Li J. Serum EGF and NGF levels of patients with brain injury and limb fracture. Asian Pacific journal of tropical medicine 2013; 6(5): 383-6.

20. Zhang R, Liang Y, Wei S. The expressions of NGF and VEGF in the fracture tissues are closely associated with accelerated clavicle fracture healing in patients with traumatic brain injury. Therapeutics and clinical risk management 2018; 14: 2315-22.

21. Schnitzler AC, Mellott TJ, Lopez-Coviella I, et al. BMP9 (bone morphogenetic protein 9) induces NGF as an autocrine/paracrine cholinergic trophic factor in developing basal forebrain neurons. The Journal of neuroscience : the official journal of the Society for Neuroscience 2010; 30(24): 8221-8.

22. Zhang R, Liang Y, Wei S. M2 macrophages are closely associated with accelerated clavicle fracture healing in patients with traumatic brain injury: a retrospective cohort study. Journal of orthopaedic surgery and research 2018; 13(1): 213.

23. Offley SC, Guo TZ, Wei T, et al. Capsaicin-sensitive sensory neurons contribute to the maintenance of trabecular bone integrity. Journal of bone and mineral research : the official journal of the American Society for Bone and Mineral Research 2005; 20(2): 257-67.

24. Apel PJ, Crane D, Northam CN, Callahan M, Smith TL, Teasdall RD. Effect of selective sensory denervation on fracture-healing: an experimental study of rats. The Journal of bone and joint surgery American volume 2009; 91(12): 2886-95. 
25. Sousa CP, Dias IR, Lopez-Pena M, et al. Bone turnover markers for early detection of fracture healing disturbances: A review of the scientific literature. Anais da Academia Brasileira de Ciencias 2015; 87(2): 104961.

26. Wijnands KA, Brink PR, Weijers $\mathrm{PH}$, Dejong $\mathrm{CH}$, Poeze M. Impaired fracture healing associated with amino acid disturbances. The American journal of clinical nutrition 2012; 95(5): 1270-7.

27. Claes L, Recknagel S, Ignatius A. Fracture healing under healthy and inflammatory conditions. Nature reviews Rheumatology 2012; 8(3): 133-43.

28. Schindeler A, McDonald MM, Bokko P, Little DG. Bone remodeling during fracture repair: The cellular picture. Seminars in cell \& developmental biology 2008; 19(5): 459-66.

29. Wijnands KA, Hoeksema MA, Meesters DM, et al. Arginase-1 deficiency regulates arginine concentrations and NOS2-mediated NO production during endotoxemia. PloS one 2014; 9(1): e86135.

30. Zhang J, Ren J, Chen H, Geng Q. Inflammation induced-endothelial cells release angiogenesis associated-microRNAs into circulation by microparticles. Chinese medical journal 2014; 127(12): 2212-7.

31. Deregibus MC, Cantaluppi V, Calogero R, et al. Endothelial progenitor cell derived microvesicles activate an angiogenic program in endothelial cells by a horizontal transfer of mRNA. Blood 2007; 110(7): 24408.

32. Deng L, Wang Y, Peng Y, et al. Osteoblast-derived microvesicles: A novel mechanism for communication between osteoblasts and osteoclasts. Bone 2015; 79: 37 42.

33. Qin Y, Wang L, Gao Z, Chen G, Zhang C. Bone marrow stromal/stem cell-derived extracellular vesicles regulate osteoblast activity and differentiation in vitro and promote bone regeneration in vivo. Scientific reports 2016; 6: 21961.

34. Zitvogel L, Regnault A, Lozier A, et al. Eradication of established murine tumors using a novel cell-free vaccine: dendritic cell-derived exosomes. Nature medicine 1998; 4(5): 594-600.

35. Valadi H, Ekstrom K, Bossios A, Sjostrand M, Lee JJ, Lotvall JO. Exosome-mediated transfer of mRNAs and microRNAs is a novel mechanism of genetic exchange between cells. Nature cell biology 2007; 9(6): 654-9.

36. Tian T, Zhu YL, Zhou YY, et al. Exosome uptake through clathrin-mediated endocytosis and macropinocytosis and mediating miR-21 delivery. The Journal of biological chemistry 2014; 289(32): 2225867.

37. Webber JP, Spary LK, Sanders AJ, et al. Differentiation of tumour-promoting stromal myofibroblasts by cancer exosomes. Oncogene 2015; 34(3): 290-302.

38. Couzin J. Cell biology: The ins and outs of exosomes. Science 2005; 308(5730): 1862-3.

39. Hao ZC, Lu J, Wang SZ, Wu H, Zhang YT, Xu SG. Stem cell-derived exosomes: A promising strategy for fracture healing. Cell proliferation 2017; 50(5).
40. Xu JF, Yang GH, Pan XH, et al. Altered microRNA expression profile in exosomes during osteogenic differentiation of human bone marrow-derived mesenchymal stem cells. PloS one 2014; 9(12): e114627.

41. Narayanan R, Huang CC, Ravindran S. Hijacking the Cellular Mail: Exosome Mediated Differentiation of Mesenchymal Stem Cells. Stem cells international 2016; 2016: 3808674.

42. Qi X, Zhang J, Yuan H, et al. Exosomes Secreted by Human-Induced Pluripotent Stem Cell-Derived Mesenchymal Stem Cells Repair Critical-Sized Bone Defects through Enhanced Angiogenesis and Osteogenesis in Osteoporotic Rats. International journal of biological sciences 2016; 12(7): 836-49.

43. Liang X, Ding Y, Zhang Y, Tse HF, Lian Q. Paracrine mechanisms of mesenchymal stem cell-based therapy: current status and perspectives. Cell transplantation 2014; 23(9): 1045-59.

44. Hu GW, Li Q, Niu X, et al. Exosomes secreted by human-induced pluripotent stem cell-derived mesenchymal stem cells attenuate limb ischemia by promoting angiogenesis in mice. Stem cell research \& therapy 2015; 6: 10 .

45. Zhang S, Chu WC, Lai RC, Lim SK, Hui JH, Toh WS. Exosomes derived from human embryonic mesenchymal stem cells promote osteochondral regeneration. Osteoarthritis and cartilage 2016; 24(12): 2135-40.

46. Golub EE. Biomineralization and matrix vesicles in biology and pathology. Seminars in immunopathology 2011; 33(5): 409-17.

47. Skog J, Wurdinger T, van Rijn S, et al. Glioblastoma microvesicles transport RNA and proteins that promote tumour growth and provide diagnostic biomarkers. Nature cell biology 2008; 10(12): 1470-6.

48. Raposo G, Stoorvogel W. Extracellular vesicles: exosomes, microvesicles, and friends. The Journal of cell biology 2013; 200(4): 373-83.

49. Gatti S, Bruno S, Deregibus MC, et al. Microvesicles derived from human adult mesenchymal stem cells protect against ischaemia-reperfusion-induced acute and chronic kidney injury. Nephrology, dialysis, transplantation : official publication of the European Dialysis and Transplant Association - European Renal Association 2011; 26(5): 1474-83.

50. Kordelas L, Rebmann V, Ludwig AK, et al. MSCderived exosomes: a novel tool to treat therapyrefractory graft-versus-host disease. Leukemia 2014; 28(4): 970-3.

51. Lobb RJ, Becker M, Wen SW, et al. Optimized exosome isolation protocol for cell culture supernatant and human plasma. Journal of extracellular vesicles 2015; 4: 27031.

52. Baglio SR, Rooijers K, Koppers-Lalic D, et al. Human bone marrow- and adipose-mesenchymal stem cells secrete exosomes enriched in distinctive miRNA and tRNA species. Stem cell research \& therapy 2015; 6: 127.

53. Peinado H, Aleckovic M, Lavotshkin S, et al. Melanoma exosomes educate bone marrow progenitor cells toward a pro-metastatic phenotype through MET. Nature medicine 2012; 18(6): 883-91. 
54. Bastian OW, Mrozek MH, Raaben M, Leenen LPH, Koenderman L, Blokhuis TJ. Serum from the Human Fracture Hematoma Contains a Potent Inducer of Neutrophil Chemotaxis. Inflammation 2018; 41(3): 1084-92.

55. Chung R, Cool JC, Scherer MA, Foster BK, Xian CJ. Roles of neutrophil-mediated inflammatory response in the bony repair of injured growth plate cartilage in young rats. Journal of leukocyte biology 2006; 80(6): 1272-80.

56. Grogaard B, Gerdin B, Reikeras O. The polymorphonuclear leukocyte: has it a role in fracture healing? Archives of orthopaedic and trauma surgery 1990; 109(5): 268-71.

57. Kovtun A, Bergdolt S, Wiegner R, Radermacher P, Huber-Lang M, Ignatius A. The crucial role of neutrophil granulocytes in bone fracture healing. European cells \& materials 2016; 32: 152-62.

58. Andrew JG, Andrew SM, Freemont AJ, Marsh DR. Inflammatory cells in normal human fracture healing. Acta orthopaedica Scandinavica 1994; 65(4): 462-6.

59. van Griensven $M$ Krettek C, Pape H. Immune Reactions after Trauma. Eur J Trauma 2003; 29: 181-92.

60. Van Griensven M Krettek C, Pape HC. Immune reactions after trauma. European Journal of Trauma 2003; 29(August): 181 - 92.

61. Stormann P, Wagner N, Kohler K, et al. Monotrauma is associated with enhanced remote inflammatory response and organ damage, while polytrauma intensifies both in porcine trauma model. European journal of trauma and emergency surgery : official publication of the European Trauma Society 2020; 46(1): 31-42.

62. Pape HC, Grimme K, Van Griensven M, et al. Impact of intramedullary instrumentation versus damage control for femoral fractures on immunoinflammatory parameters: prospective randomized analysis by the EPOFF Study Group. The Journal of trauma 2003; 55(1): 7-13.

63. Hesselink L, Bastian OW, Heeres M, et al. An increase in myeloid cells after severe injury is associated with normal fracture healing: a retrospective study of 62 patients with a femoral fracture. Acta orthopaedica 2018; 89(5): 585-90.

64. Bastian OW, Kuijer A, Koenderman L, et al. Impaired bone healing in multitrauma patients is associated with altered leukocyte kinetics after major trauma. Journal of inflammation research 2016; 9: 69-78.

65. Schmidt-Bleek K, Schell H, Schulz N, et al. Inflammatory phase of bone healing initiates the regenerative healing cascade. Cell and tissue research 2012; 347(3): 567-73.

66. Garley M, Jablonska E. Heterogeneity Among Neutrophils. Archivum immunologiae et therapiae experimentalis 2018; 66(1): 21-30.

67. Lund-Johansen F, Terstappen LW. Differential surface expression of cell adhesion molecules during granulocyte maturation. Journal of leukocyte biology 1993; 54(1): 47-55.

68. Soligo D, Schiro R, Luksch R, et al. Expression of integrins in human bone marrow. British journal of haematology 1990; 76(3): 323-32.
69. Deniset JF, Kubes P. Recent advances in understanding neutrophils. F1000Research 2016; 5: 2912.

70. Deniset JF, Kubes P. Neutrophil heterogeneity: Bona fide subsets or polarization states? Journal of leukocyte biology 2018; 103(5): 829-38.

71. Looney MR, Thornton EE, Sen D, Lamm WJ, Glenny RW, Krummel MF. Stabilized imaging of immune surveillance in the mouse lung. Nature methods 2011; 8(1): 91-6.

72. Wang J, Hossain M, Thanabalasuriar A, Gunzer M, Meininger C, Kubes P. Visualizing the function and fate of neutrophils in sterile injury and repair. Science 2017; 358(6359): 111-6.

73. Kim JH, Podstawka J, Lou Y, et al. Aged polymorphonuclear leukocytes cause fibrotic interstitial lung disease in the absence of regulation by $\mathrm{B}$ cells. Nature immunology 2018; 19(2): 192-201. 


\section{Summary}

Martijn Hofman 
In this thesis, we focused on several aspects of neuro-humoral modulation of fracture healing. We explored the literature, performed clinical studies and executed several experimental studies on small animal models.

The aim of chapter 1 was to provide solid background information on the inflammatory and neuro-humoral responses to fracture healing and on fracture healing in general to equip the reader with a good basis to assimilate the information within this thesis. This is achieved by explaining the normal fracture healing process as well as its different stages and by clarifying the role of angiogenesis and different biologic factors in this process. Moreover, the disturbed fracture healing process along with its consequences and the influence of poly- and neuro-trauma on fracture healing are described. The literature on these topics clearly shows that fracture healing is an immense and complex phenomenon and many of its aspects are yet to be resolved. Therefore, the drive to write this thesis was the need to clarify little parts of the missing pieces in the realm of the fracture healing mechanism.

\subsection{PART I: INFLUENCE OF TBI ON FRACTURE HEALING}

In chapter $\mathbf{2}$ and $\mathbf{3}$, we aimed to provide an answer to the hypothesis that concomitant TBI enhances fracture healing.

In the literature review presented in chapter 2, we included clinical, in vivo and in vitro studies to test this hypothesis, which is represented by expert opinions in the field. In these studies, performed in the last 50 years up to 2013, many different substances are investigated, such as cytokines, growth factors, mesenchymal stem cells, genes, hormones, proteins and enzymes, but a concrete pathomechanism to confirm the hypothesis was not find. However, the consensus of all published preclinical papers is that TBI indeed accelerates fracture healing via the osteoinductive factors in the serum and CSF released by the injured brain. On the contrary, the clinical studies with relatively small populations cannot fully support this hypothesis.

In chapter 3, we performed a retrospective study to identify the roles of chest trauma, TBI and fracture stabilisation strategies on fracture healing. Although the results of this study could not confirm the relation between TBI or chest injury and fracture healing tendency, we demonstrated that the number of operations performed in patients with long-bone fractures was an independent predictor for non-union development.

The results of these two studies were an incentive to further elaborate on our research on fracture healing with a focus on neuro-humoral pathways.

\subsection{PART II: NEURO-HUMORAL MODULATION OF FRACTURE HEALING}

In chapter 4, we aim to specify the proved influence of efferent sensory nerves on fracture healing by the selective blocking of the neurokinin-1-tachykinin (NK1) receptor for substance $\mathrm{P}$ as a neurotransmitter of these efferent sensory nerves. In this study, we showed that the blockage of this specific neurotransmitter impaired the gene expression of important osteogenic proteins (i.e. osteocalcin, collagen 1A2 and collagen 2A1) in the early stages of fracture healing. Furthermore, we demonstrated that the blockage of the NK1 receptor resulted in the decreased biomechanical strength of the callus/bone throughout the fracture healing process in a small animal fracture model. 
Chapter 5 reverts to a study we performed on gait pattern and muscle weight in a small animal femur fracture model. Because gait analysis is becoming increasingly important in the evaluation and rehabilitation of patients with lower extremity fractures, we wanted to establish a standard gait analysis method to investigate the gait parameters in the most used experimental fracture healing model: the small animal model. Normal gait pattern and muscle weight evolvement during fracture healing in rats was outlined in this chapter. We successfully introduced the CatWalk $^{T M}$ system for gait analysis in small animal models. Moreover, we demonstrated that this system could adequately register both static and dynamic gait parameters and that the gait pattern evolvement in this small animal model resembles that in patients with lower extremity fractures.

In chapter 6, we clinically evaluated the biomarkers of the arginine-NO metabolism in trabecular bone harvested during RIA procedures for the non-union treatment of long bones. We demonstrated for the first time that arginine and NOS2 can be used as prognostic biomarkers to predict a successful outcome of non-union treatment.

In chapter 7, 8 and 9, our focus shifted towards the role of cellular mechanisms within the inflammation response to femoral fracture in a small animal model.

In chapter 7, the influence of MVs, which play an important role in intercellular and transcellular signal transduction, on osteoblasts was investigated. We showed that systemically derived MVs isolated after femoral fracture were time-dependently incorporated in osteoblasts and concentrated around their nucleus. Furthermore, we demonstrated that MVs potentially affect fracture healing regulation by increasing the viability of osteoblasts, particularly in the later inflammatory phases of fracture healing.

In chapter 8, we demonstrated that three days after fracture induction, a temporary reduction in neutrophil count occurred, along with a concurrent increase of CD11a expression and a concurrent decrease of CD11b expression on circulatory neutrophil membranes. Moreover, we showed that during the subsequent restoration of neutrophil homeostasis, a novel subset of CD11 $\mathrm{b}^{\text {high }} / \mathrm{CD} 11 \mathrm{a}^{\text {high }}$ neutrophils appears, with a co-expression of $\alpha_{4}$-integrin (CD49d).

In chapter 9, we investigated the impact of an intramedullary stabilised femur fracture on the characteristics of the pulmonary neutrophil pool in a small animal model. Contrary to the decreased neutrophil activation in peripheral blood after trauma demonstrated in chapter 8 , the number of neutrophils in the pulmonary pool and the expression of membrane receptors transiently increased in the early inflammatory phases of fracture healing. Thereafter, the values again normalised to physiological levels.

Chapter 10 includes the general discussion and conclusions of this thesis, in which the results of the separate studies are discussed in view of the current knowledge represented in the scientific literature. In addition, the gaps in the obtained knowledge are identified, which could be explored in future studies. 


\section{Summary in Dutch}

Martijn Hofman 
In dit proefschrift werden verschillende aspecten van neuro-humorale modulatie van fractuurheling onderzocht. We verkenden de literatuur en voerden zowel klinische als dierexperimentele studies uit.

Het doel van hoofdstuk 1 was de lezer van voldoende achtergrond informatie betreffende de inflammatoire en neuro-humorale respons op fractuur heling te voorzien en een goede basis te verschaffen voor het verwerken van de informatie in dit proefschrift. Daarvoor hebben we ten eerste het normale proces van fractuurheling met zijn verschillende stadia verklaard en vervolgens de rol van angiogenese en verschillende biologische factoren aangehaald. Ook werd de invloed van poly- en neuro-traumata op de fractuur heling en het proces en de consequenties van een verstoorde fractuurgenezing belicht. Uit het literatuuronderzoek van deze onderwerpen is gebleken dat fractuurheling op zichzelf een immens complex fenomeen vormt, waarvan nog steeds vele aspecten niet zijn opgehelderd. Onze grote drijfveer voor dit proefschrift was dan ook het ophelderen van kleine vraagstukken betrekking hebbend op ontbrekende stukjes van het mechanisme van fractuurheling.

\section{DEEL I: INVLOED VAN TRAUMATISCH HERSENLETSEL OP FRACTUURHELING}

In hoofdstuk $2 \& 3$ hebben we getracht de hypothese dat begeleidend hersenletsel het proces van fractuurheling positief beïnvloed te toetsen.

In de literatuurstudie, zoals beschreven in hoofdstuk 2, werden zowel klinische als ook in vivo en in vitro studies geïncludeerd, om deze hypothese, die door experts in de klinische medische wereld vertegenwoordigd wordt, te testen. In deze studies, uitgevoerd in de laatste vijftig jaar tot aan 2013, werden veel verschillende substanties onderzocht, zoals cytokinen, groeifactoren, mesenchymale stamcellen, genen, hormonen, proteïnen, enzymen, enzovoort. Ondanks dat we een concreet pathomechanisme om de hypothese te bevestigen niet konden vinden, is de consensus binnen de gepubliceerde preklinische studies dat traumatisch hersenletsel daadwerkelijk de fractuurheling positief beïnvloed via door het brein vrijgezette osteoinductieve factoren in het serum en de liquor. Aan de andere kant, wordt deze consensus niet gedeeld door de resultaten van de relatief kleine klinische studies, die we geëvalueerd hebben.

In hoofdstuk $\mathbf{3}$ werd een retrospectieve klinische studie uitgevoerd om de invloed van thorax trauma, traumatisch hersenletsel en de fractuurstabilisatie-strategie op de fractuurgenezing te beoordelen. Ondanks dat de resultaten van deze studie geen relatie tussen traumatisch hersenletsel of thorax trauma en fractuurheling konden aantonen, kwam het aantal operaties uitgevoerd in patiënten met lange pijpbeenderfracturen naar voren als een onafhankelijke risicofactor voor het ontwikkelen van een non-union.

De resultaten van deze twee studies waren een aanzet om ons onderzoek naar fractuurheling uit te breiden met een focus op de invloed van neuro-humorale regelsystemen.

\section{DEEL II: NEURO-HUMORALE MODULATIE VAN FRACTUURHELING}

Het doel van het onderzoek, beschreven in hoofdstuk 4 was de bewezen invloed van efferente zenuwsignalen op de botheling te specificeren. Daarvoor hebben we in een dierexperimenteel fractuurmodel de neurokinine-1-tachykinine receptor voor 
substance $\mathrm{P}$, als neurotransmitter van deze efferente sensibele zenuwbanen, geblokkeerd. In deze studie konden we aantonen dat deze selectieve blokkade de genexpressie van belangrijke osteogene proteïnen, te weten osteocalcine, collageen $1 \mathrm{~A} 2$ en collageen $2 \mathrm{~A} 1$, in de vroege fase van de fractuurheling reduceert. Bovendien konden we aantonen dat deze blokkade de biomechanische sterkte van de callus / het bot verzwakt gedurende het gehele fractuurhelingsproces.

Hoofdstuk 5 handelde over gang- en spieratrofie-analyses uitgevoerd in ons dierexperimenteel fractuurmodel. Omdat ganganalyses steeds belangrijker worden in de evaluatie en revalidatie van patiënten met fracturen aan de onderste extremiteiten, wilden we een standaard ganganalyse methode tot stand brengen en valideren voor het meest gebruikte dierexperimentele fractuurmodel binnen de wetenschap. We konden succesvol het CatWalk ${ }^{T M}$-system voor ganganalyses van klein diermodellen introduceren en demonstreren dat deze methode adequaat zowel statische als dynamische parameters kan registreren en dat het patroon van de ganganalyse binnen dit diermodel vergelijkbaar is met het patroon van de ganganalyses bij patiënten na fracturen van de onderste extremiteit.

In hoofdstuk 6 hebben we een klinische evaluatie uitgevoerd van bio-markers van het arginine-stikstofoxide metabolisme in trabeculair bot. Het weefsel werd verkregen door middel van RIA-procedures voor de behandeling van non-unions in patiënten. We konden voor het eerst aantonen dat arginine en NOS2 voorspellende waarde hadden voor een succesvolle behandeling van non-unions.

In hoofdstuk 7, 8 \& 9 verschoof de focus van ons onderzoek naar de rol van cellulaire mechanismen binnen de inflammatoire reactie op femurfracturen in ons dierexperimenteel model.

Het onderzoek naar de invloed van microvesikels, die een belangrijke rol vervullen in de inter- en transcellulaire signaal transductie, op osteoblasten werd beschreven in hoofdstuk 7. We konden laten zien dat de na een femurfractuur vrijgezette systemische microvesikels tijdsafhankelijk geïncorporeerd werden in osteoblasten en dat zij zich concentreerden rondom de celkern. Verder konden we aantonen microvesikels potentieel de regulatie van de fractuurheling beïnvloeden kunnen, door de levensvatbaarheid van deze osteoblasten te vergroten, voornamelijk in de latere inflammatie-fases van de fractuurgenezing.

In hoofdstuk 8 demonstreerden we dat 3 dagen na de fractuur inductie een tijdelijke afname van het aantal neutrofielen optrad, vergezeld van een stijging van de CD11a expressie en een daling van de CD11b expressie op de membraan van neutrofielen in de circulatie. Bovendien konden we aantonen dat gedurende het daaropvolgend herstel van de neutrofielen homeostase een nieuwe subset van CD11bigh $/$ CD11 $\mathrm{a}^{\text {high }}$ neutrofielen ontstaat, die een co-expressie met $\alpha_{4}$-integrin (CD49d) laten zien.

Het onderzoek naar de impact van een intramedullair gestabiliseerde femurfractuur op de eigenschappen van de pulmonaire neutrofielen pool in ons dierexperimenteel model werd in hoofdstuk 9 beschreven. In tegenstelling tot de afname van neutrofielen-activiteit in het perifere bloed na het trauma, zoals beschreven in hoofdstuk 8, nam zowel het aantal neutrofielen in de pulmonaire pool, alsook de expressie van membraanreceptoren tijdelijk toe in de vroege inflammatoire fases 
van het fractuurhelingsproces. Hierna normaliseerden deze waarden weer tot het fysiologische niveau.

Hoofdstuk 10 bevat de algehele discussie en de conclusies van dit proefschrift, waarin de resultaten van de afzonderlijke studies bediscussieerd worden in het licht van de huidige kennis uit de wetenschappelijke literatuur. Verder worden hiaten in de opgedane kennis geïdentificeerd, die mogelijk uitgediept kunnen worden in toekomstig onderzoek. 


\section{Summary in German}

Martijn Hofman 
Die vorliegende Dissertation untersuchte verschiedene Aspekte der neurohumoralen Modulation im Hinblick auf die Frakturheilung. Hierzu wurden sowohl klinische als auch experimentelle Studien vor dem Kontext der internationalen Kenntnislage durchgeführt.

Kapitel 1 der vorliegenden Dissertation widmete sich dem zentralen Aspekt der Hintergrundanalyse der inflammatorischen als auch neurohumoralen Immunantwort hinsichtlich der Frakturheilung, um eine generelle Grundlageninformation $\mathrm{zu}$ bieten. So werden der normale Ablauf der Knochenheilung als auch die unterschiedlichen Stadien in Abhängigkeit der Angiogenese und verschiedener biologischer Faktoren dargelegt. Gleichzeitig werden die gestörte Knochenheilung mit ihren Auswirkungen sowie die Beeinflussung durch Polytrauma oder Neurotrauma beschrieben. Im Kontext zur internationalen Literatur wird deutlich, dass die Frakturheilung ein umfassendes und komplexes Phänomen mit bislang zahlreichen, ungeklärten Aspekten darstellt. Zentrales Ziel dieser Dissertation war daher, bislang ungeklärte Zusammenhänge der Frakturheilungsmechanismen weiterführend zu untersuchen.

\section{TEIL I: EINFLUSSNAHME DES SCHÄDELHIRNTRAUMAS (SHT) AUF DIE FRAKTURHEILUNG}

In Kapitel 2 und 3 der vorliegenden Dissertation wurde die Hypothese überprüft, ob ein begleitendes SHT die Knochenheilung generell fördert.

In Kapitel 2 wird nun eine Literaturanalyse im Sinne eines Reviews vorgelegt, die klinische, in vivo sowie in vitro Studien zur Überprüfung dieser Hypothese umfasste. Zahlreiche eingeschlossene Studien der letzten 50 Jahre bis einschließlich 2013, die Cytokine, Wachstumsfaktoren, mesenchymale Stammzellen, Geneuntersuchungen, Hormone, Proteine und Enzyme analysierten, konnten die aufgeführte Hypothese nicht belegen. Im Rahmen experimenteller Studien scheinen zentral ausgeschüttete osteoinduktive Faktoren, die im Serum und Liquor nach SHT nachweisbar sind, die Frakturheilung zu beschleunigen. Demgegenüber konnten klinische Studien einen solchen Effekt jedoch nicht belegen.

Daher wurde in Kapitel 3 der Dissertation eine retrospektive, klinische Studie durchgeführt, um den Einfluss des Schädelhirntraumas, des Thorax-traumas sowie der operativen Frakturversorgung auf die Frakturheilung zu untersuchen. Wenngleich die Ergebnisse dieser Studie einen Zusammenhang zwischen SHT, Thorax-trauma und Frakturheilung nicht belegen konnten, so wurde die Anzahl an operativen Eingriffen an langen Röhrenknochen als unabhängiger Risikofraktur für die Entwicklung einer Pseudarthrose eruiert.

Beide Studien dienten als Grundlage und Incentive im Hinblick auf die nachfolgenden Studien zur Frakturheilung.

\section{TEIL II: NEUROHUMORALE MODULATION DER FRAKTURHEILUNG}

Kapitel 4 der Dissertation galt der Spezifizierung der Einflussnahme efferenter sensorischer Nerven auf die Frakturheilung, wobei der Neurokinin-1-Tachykinin Rezeptor für den Neurotransmitter Substanz P blockiert wurde. Aus dieser Blockade resultierte eine nachgewiesene Beeinträchtigung der Genexpression bedeutsamer osteogener Proteine (z.B. Osteocalcin, Kollagen 1A2 und 2A1) während der 
Frühphase der Frakturheilung. Darüber hinaus führte die Blockade des NK1Rezeptors im Kleintiermodell zu einer biomechanischen Schwächung des Kallus über den gesamten Knochenheilungsprozess hinaus.

Kapitel 5 stellte eine Studie des Kleintiermodells an Ratten dar, welche im Rahmen eines Femur-Frakturmodells Gang- und Muskelanalysen durchführte. Basierend auf der Tatsache, dass Ganganalysen im rehabilitativen Behandlungsbereich bei Patienten mit Verletzungen der unteren Extremitäten zunehmend an Bedeutung gewinnen, sollte diese Studie eine Standardmethode zur Ganganalyse nach Femurfraktur im Kleintiermodell etablieren. Sowohl eine normale Entwicklung des Gangbildes als auch die regelhafte Zunahme der Muskelmasse während der Frakturheilung im Kleintiermodell werden in diesem Kapitel dargelegt. Die Einführung des CatWalk ${ }^{\mathrm{TM}}$-Systems zur Ganganalyse konnte zuverlässig sowohl statische als auch dynamische Parameter erfassen und somit eine experimentelle Option zum zukünftigen Vergleich der Gangentwicklung bei Patienten mit Verletzungen der unteren Extremitäten bieten.

In Kapitel 6 der vorliegenden Dissertation wurden in einer klinischen Studie Biomarker des Arginin-Stickstoffmonoxid Metabolismus aus dem intramedullären Knochen analysiert, die während der Markraumbohrung (RIA-Verfahren) in der Pseudarthrosenversorgung gewonnen wurden. Es gelang hierbei erstmals der Nachweis, dass Arginin, Ornithin und NOS2 als Prognosemarker im Hinblick auf die Pseudarthrosenheilung valide genutzt werden können.

Kapitel 7, 8 und 9 legten den Fokus auf die zellulären Regulationsmechanismen der inflammatorischen Reaktion nach Femurfraktur im Kleintiermodell.

In Kapitel 7 wurde der Einfluss von Mikrovesikeln, die eine bedeutsame Rolle in der inter- und transzellularen Kommunikation einnehmen, auf Osteoblasten analysiert. So konnte nachgewiesen werden, dass die abgeleiteten Mikrovesikel nach Femurfraktur zeitabhängig vermehrt in Osteoblasten aufgenommen und konzentriert um den Zellkern gelagert vorlagen. Zudem scheint die Viabilität der Osteoblasten durch die Inkorporation der Mikrovesikel beeinflusst zu werden, was insbesondere in der späten inflammatorischen Phase die Frakturheilung begünstigt.

In Kapitel 8 wurde dargelegt, dass drei Tage nach Frakturinduktion eine temporäre Reduktion Neutrophiler unter gleichzeitiger Zunahme einer CD11a Expression bei Abfall einer CD11b Expression auf den Zelloberflächen der zirkulierenden Neutrophilen auftrat. Zudem imponierte eine neue Untergruppe von CD11b high/CD11ahigh Neutrophilen mit Co-Expression von $\alpha 4$-Integrin (CD49d) während der darauffolgenden Wiederherstellung der Neutrophilen-Hämostase.

Kapitel 9 widmete sich dem Einfluss einer intramedullär stabilisierten Femurfraktur auf Charakteristika pulmonaler Neutrophiler im Kleintiermodell. Im Gegensatz zu der in Kapitel 8 beschriebenen, reduzierten Aktivität Neutrophiler im peripheren Blut stieg die Anzahl Neutrophiler und die Expression der Membranrezeptoren im pulmonalen Pool während der frühen Phase der Frakturheilung deutlich an. Abschließend fielen diese wieder auf das Normalniveau ab. 
Kapitel 10 beinhaltet letztlich eine umfassende Diskussion und Schlussfolgerung der vorgelegten Dissertation. Die Ergebnisse der einzelnen Untersuchungen werden vor dem Hintergrund der international etablierten Studien ausführlich diskutiert. Zudem können bestehende Diskrepanzen für zukünftige Studien aufgezeigt werden. 


\section{Valorisation}

Martijn Hofman 
As presented in the introduction of this thesis, the process of fracture healing and bone regeneration is an extremely complex process in which, besides the musculoskeletal system, other systems such as the neurological, vascular and immune systems play an important role. An immense amount of biological factors from each of these systems interact with each other and contribute to the regeneration of bony defects. With this thesis, we aimed to evaluate the influence of neuro-humoral modulation in the fracture healing process, without pretending that the evaluation is complete, hence to just lift a tip of the veil.

The valorisation of results from experimental research, in the way Karl Marx originally conceptualised this theoretical concept of 'Verwertung' in his critique of political economy, is not that obvious because the results of experimental studies often cannot directly be implemented in daily lives or in medical treatments and thus will not generate money. Therefore, in this valorisation, we describe the value our results have or could have for further research and possibly in the long run for clinical applications.

The results from our review described in chapter 2, the retrospective study described in chapter 3 and the results of the literature study described in the general discussion (chapter 10) about newer studies on the impact of TBI on fracture healing are all concerned with the influence of TBI on the fracture healing process. On one hand, we could demonstrate that the majority of the experimental studies tend to provide evidence for the positive influence of TBI on fracture healing; on the other hand, the clinical studies, most of which are retrospective, could not generally subscribe these findings. The value of these results should be such that the scientific community is triggered to further investigate this phenomenon and discover the pathophysiologic mechanisms that explain the relation between certain biologic factors released after TBI and the bone regeneration process. Moreover, the results shown in our studies could be an impulse to instigate a large randomised, prospective, clinical study to find clinical support for these experimental findings.

In chapter 4, we demonstrated that the blockage of the NK1-receptor for substance $P$ has a considerable influence on gene expression and bone strength throughout the fracture healing process. A logical next step in the research on the influence of neurotransmitters in fracture healing would be to administer substance P in small animal fracture healing and/or disturbed fracture healing models to evaluate its direct influence on the fracture healing process. Substance $\mathrm{P}$ is a biomarker, which is set free after TBI; in addition to causing pro-inflammatory effects, it causes increased vascular permeability, brain oedema and functional deficits after TBI ${ }^{1}$. Lorente et al. showed that the substance $P$ levels in serum are correlated to the severity and mortality of TBI ${ }^{2}$. In this context, it would be very interesting to evaluate the dynamics of substance $P$ and fracture healing in a small animal fracture model with concomitant TBI. After further experimental research on the function of substance $P$, it also could become a biomarker for fracture healing in patients because the concentration of substance P might be associated with the quality of fracture healing.

In chapter 5, we proved the CatWalk gait analysis system to be an outstanding tool to assess both static and dynamic gait parameters in a non-invasive, longitudinal manner in an experimental small animal model of fracture healing. In our opinion, the CatWalk system has the potential to become the gold standard for gait analyses 
in small animal fracture models. Because more than 50\% of experimental animal fracture models are performed with mice and rats, the use of this system would significantly improve the knowledge about behavioural and locomotor recovery after lower extremity fractures.

To date, the most important tools for diagnosing disturbed fracture healing are clinical and radiological findings. Although research on possible biomarkers that can be used as predictors for non-union development is promising, achieving consensus is very difficult because the evidence available is heterogeneous ${ }^{3}$. The results of our study described in chapter 6 most importantly indicate a significantly lower ornithine concentration and arginase-1 expression in the bone marrow of patients developing non-unions. This was the first study to recognise these possible biomarkers (i.e. arginine, ornithine and iNOS) that could be used as predictors of the outcome of the autologous bone grafting procedure by RIA in cases of non-union. As the exact treatment and the time point of this non-union treatment are still under discussion, and the socio-economic impact of disturbed fracture healing is immense, finding biomarkers, which can predict the prognosis of the fracture healing process and the outcome of certain procedures performed in cases of non-unions, will be very valuable. Therefore, this study could be an excellent starting point to further investigate these biomarkers in the fracture healing process and to determine certain cut-off points for the different biomarkers, based on which the prognosis of fracture healing and non-union treatment could be estimated.

During the last years, the intercellular communication through vesicles loaded with different proteins, mRNAs and miRNAs, known as exosomes, MVs, and apoptotic bodies, is gaining interest in fracture healing research. In this context, we showed in an in vitro study, described in chapter $\mathbf{7}$, that MVs isolated after a femoral fracture were time-dependently incorporated in osteoblasts and concentrated around the nucleus. These MVs from trauma plasma increased the proliferation and viability of osteoblasts, particularly in the late phase (i.e. two weeks post-fracture) of fracture healing. The fracture healing process involves a complex network of signal transduction between a variety of cells. Demonstrating the regulating effect of MVs on fracture healing by increasing the proliferation and viability of osteoblasts is just a first step in understanding the role that MVs might play in the fracture healing process. The characteristics of the exact role of MVs in the intercellular communication between cells in the fracture healing process are still to be discovered in future research, including the origin, the composition and the target cells of these MVs as well as the mechanism of action of the different constituents within these MVs. If these questions are answered, the way in which the responsible contents of MVs can be used to help in the diagnostics or treatment of fractures should be addressed. Possible applications could be the adjustment of the content or local application of MVs to alter the fracture healing process. Because the current exosome isolation methods such as ultracentrifugation and ultrafiltration only provide a low exosome yield ${ }^{4}$, the challenge of obtaining sufficient amounts of exosomes should be overcome.

In our small animal neutrophil studies, demonstrated in chapter 8 and 9, we showed that three days after intramedullary nailing and fracture induction, the concentration 
of circulating neutrophils as well as the neutrophil activation, characterised by a change of integrin expression on their surface, decreased. One theory explaining this decrease is that the decrease expresses the homing of neutrophils into the fracture haematoma during the inflammatory stage of fracture healing, wherein they clear fracture debris and initiate further steps in the normal fracture healing process. Another theory, supported by our findings in chapter 9, is that this decrease is attributable to an increased extravasation of neutrophils not only into the fracture haematoma but also into peripheral tissues, such as the lungs, potentially causing tissue damage (i.e. ARDS/ALI). In future studies the number of circulatory neutrophils should be compared to the number of neutrophils in the fracture haematoma and the pulmonary pool after fracture. All in all, the total range of functions of the neutrophils as activators and regulators of different cell processes is still to be discovered in future studies.

We also demonstrated, in chapter 8, the increased heterogeneity of the blood neutrophil pool during the restoration phase after fracture, with a new subset of unique $\mathrm{CD} 11 \mathrm{~b}^{\text {high }} / \mathrm{CD} 11 \mathrm{a}^{\text {high }}$ neutrophils present in the post-inflammatory neutrophil pool. Because it is not yet clear if these subsets belong to separate developing lineages or embody certain activation states of a common precursor, this finding offers a basis for further research on the exact origin of this novel subtype and on novel immunotherapeutic strategies to modulate neutrophil homeostasis after fractures or in cases of disturbed fracture healing.

Similarly to several former studies that have shown the increased pulmonary neutrophil influx after trauma, in chapter 9, we demonstrated a transient increase in pulmonary neutrophil deposition and a contemporary increase in the activation status of the pulmonary neutrophil pool after an intramedullary stabilised femur fracture. Furthermore, we showed the striking differences in the activation status of the neutrophils belonging to the pulmonary parenchymal compartment and those belonging to the broncho-alveolar compartment. This qualitative description of pulmonary neutrophil populations and their characteristics should incite further research on the influence of different neutrophil subsets on the development of pulmonary complications and on the possibility to use these neutrophil cell-surface receptors as markers for neutrophil activation status.

\section{REFERENCES}

1. Donkin JJ, Nimmo AJ, Cernak I, Blumbergs PC, Vink R. Substance $P$ is associated with the development of brain edema and functional deficits after traumatic brain injury. Journal of cerebral blood flow and metabolism : official journal of the International Society of Cerebral Blood Flow and Metabolism 2009; 29(8): 1388-98.

2. Lorente L, Martin MM, Almeida T, et al. Serum substance $P$ levels are associated with severity and mortality in patients with severe traumatic brain injury. Critical care 2015; 19: 192.
3. Sousa CP, Dias IR, Lopez-Pena M, et al. Bone turnover markers for early detection of fracture healing disturbances: A review of the scientific literature. Anais da Academia Brasileira de Ciencias 2015; 87(2): 1049-61.

4. Peinado H, Aleckovic M, Lavotshkin S, et al. Melanoma exosomes educate bone marrow progenitor cells toward a pro-metastatic phenotype through MET. Nature medicine 2012; 18(6): 883-91 


\section{Acknowledgements}


Last but not least, the word of thanks for those people who supported, inspired, and helped me on my way to become a surgeon and finally, a Doctor of Philosophy.

Prof. Dr. med. Frank Hildebrand, dear Frank, thank you very much for supporting me throughout the last years of this PhD-project and for giving me the opportunity to combine my research with my daily work as a trauma surgeon within your department. You were very important in critically reviewing and improving my manuscripts and providing ideas of how to design and mould the theme of this project. Thank you for your constructive cooperation and your attention.

Prof. dr. Martijn Poeze, dear Martijn, you gave me the opportunity to become a PhDcandidate at the MUMC+ and although it was sometimes difficult to organize our meetings due to our busy schedules, these meetings were always very gratifying. You were very helpful in structuring and outlining this thesis and I am grateful and proud to be the first Doctor of Philosophy derived from the bilateral co-operation of our two departments. Hopefully, in the near future, we can expand this collaboration both in the clinical and the research field.

Priv. Doz. Dr. med. Hagen Andruszkow, dear Hagen, I would not have been able to adequately evaluate the results of all studies without your help and your enthusiasm about statistics and therefore, I thank you from the bottom of my heart. You always made time for me and you patiently revised every version of my manuscripts with me, even when we reached 'versie 29' of a single manuscript.

Johannes Greven. As head of the research laboratory of our department, you were the spider in the web of all studies within my PhD-project. Thank you for your cooperation, your perseverance and your coordinating role in this project.

Furthermore, I want to thank Michel Teuben, Zhi Qiao, Dennis Meesters and all other research colleagues for their collaboration, help and support, especially at the beginning and end of this PhD-project.

dr. Heinrich Janzing, dear Heinrich, maybe you are not aware of the great impact you had on me as a person and the role model you were to me as a surgeon. For me, you were the founding father of the way I would like to be as a physician, a surgeon and a tutor and therefore I am truly grateful that I had the privilege to work with and learn from you. Thank you for being a great inspiration and motivation for me to become a trauma surgeon.

dr. Jan Verbruggen, dear Jan, thank you for introducing me in Aachen with PD. Dr. med. Hans-Josef Erli, so I could still aspire to become a trauma surgeon, which was one of my dreams, after all. Until now, I have no regrets!

Dr. med. Mathias Nossek, dear Mathias, I have never met a colleague who worked so hard and was as correct to patients and colleagues as you were. In that, you were a great example to me and besides that, I have learned a lot from you operatively.

Prof. Dr. med. Bernhard Schmidt-Rohlfing, dear Bernhard, during my residency you enabled me to operate a lot and therefore I could gather a lot of experience, for which I am truly thankful.

Dr. med. Achim Dohmen, dear Achim, I got to know you during my orthopaedic rotation at the St. Elisabeth Hospital Geilenkirchen, where you teached me how to 
implant an endoprosthesis properly and efficiently. Furthermore, I greatly appreciate your drive to expand your department and to warrant the quality of patient care. Thank you for the great time I had in Geilenkirchen with you.

Priv. Doz. Dr. med. Richard Sellei, dear Richard, thank you for taking care of me during my first period in Germany and for being a great colleague to me throughout the years. I deeply respect you as a friend and as a surgeon and by the way, I still strive for a little piece of your patience.

Prof. Dr. med. Philipp Kobbe, dear Philipp, thank you for being a sparring partner in discussions about the treatment of patients, future perspectives and other (less) important things in life. These discussions always force me to be on the alert, and that is a good thing.

In addition, I would like to thank my dear friends and family, who provided a sympathetic ear, stimulating discussions as well as happy distractions to rest my mind outside of my research and work. Special thanks go out to...

dr. Guido Koopmans, dear Guido, dear best friend, thank you for being my sounding board throughout this project, because that helped me a lot, just by talking about science. I greatly appreciate you as my best friend and I deeply respect your perseverance in organising and structuring your research projects at Algiax pharmaceuticals around your promising compound AP-325. I am really grateful that you are my friend and deep inside, I envy you for the time you spend on sports, of course!

My dear brother, Jeroen, although we do not see each other very often, I know I can always rely on you and that is a comforting thought. Thank you.

Dear dad, until now I have never met another person who is interested in so many fields of life, nature, sports, languages, countries, people, etc. You largely formed my character, which I have inherited from mom and I want to thank you that you always encouraged and supported me without conditions in everything I did!

Dear mom, thank you for always covering my back and supporting me in whatever my goals were. Above all, to me, you are the one who gives unconditional motherly love to me and I am really grateful that my children receive the same!

Dear Jente, Max, \& Cato, my three precious 'kabouters', although I work a lot, and I can be a little grumpy sometimes, you may never forget that I love all three of you just like you are and I will do anything to enable you to freely strive for your goals and to become a happy person. I could never wish for nicer, funnier, and sweeter children as you are!

My dear Geertje, since I know you, my morning moodiness has nearly disappeared. I want to thank you that I could always rely on you as the base of our family. Honestly, without your support and the freedom you give to me, I could not have reached so far. You are the love of my life, whatever happens next, and I hope, some time I can make up for my absences and long working hours. I love you! 


\section{Curriculum vitae}


Martijn was born on the 30th of September 1976 in Sittard, the Netherlands. He grew up in a sportive family and during the first 20 years of his life playing soccer was his greatest passion. Since a class 'human biology' in the last year of his grammar school, he knew that if he did not manage to become a professional soccer player, he wanted to become a physician.

In 1994 he finished his secondary school and started to study human medicine at the 'Limburgs Universitair Centrum' in Diepenbeek, Belgium. He combined his part-time study with playing soccer and here, thanks to the outstanding anatomy courses, he decided that he wanted to work in a surgical

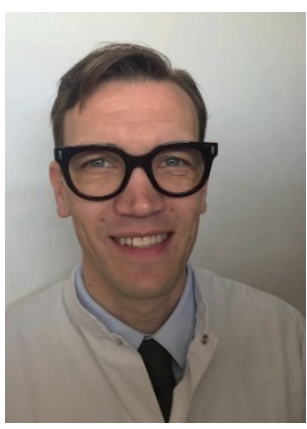
specialty. In 1997 he switched from the Belgian university to the Maastricht University in the Netherlands, because there he could combine his sporting career with a full-time medical study.

After his studies, he worked from 2003 - 2007 in different hospitals in the Netherlands, among others in the VieCuri Medical Centre in Venlo, where under supervision of dr. H.M.J. Janzing his basic surgical skills were formed. As he did not manage to get into the surgical residency program within the Netherlands, and he was determined to become a trauma surgeon, he diverted to the other neighbouring country, Germany. Here he completed his residency 'Orthopaedics and Trauma Surgery' at the University Medical Center RWTH Aachen under the supervision of Priv. Doz. Dr. med. H.J. Erli und Prof. Dr. med. F.U. Niethard and the St. Elisabeth Hospital Geilenkirchen under supervision of Dr. med. A. Dohmen.

Since 2011 he became an attending physician of the department of Orthopaedic Trauma and Reconstructive Surgery at the University Medical Center RWTH Aachen, Germany. He also became the head of the department of Sports Traumatology and Minimal-invasive Joint Surgery. Prof. Dr. med. H.C. Pape, as head of the department provided the circumstances for him to fully develop his traumatological skills and to extend the department freely. After Prof. Dr. med. F. Hildebrand took over the position as head of the department, Martijn gained a leading position within the department of Orthopaedic Trauma and Reconstructive Surgery at the University Medical Center RWTH Aachen in September 2018.

In 2015 he started, parallel to his daily work as a physician, this Ph.D.-project in cooperation with the department of Trauma Surgery of the Maastricht University Medical Center (MUMC+). Supported by his colleagues of the research laboratory of the department of Orthopaedic Trauma and Reconstructive Surgery at the University Medical Center RWTH Aachen, he could complete his thesis in the summer of 2020.

Also due to the obvious ups and downs of a scientific research project as this, Martijn worked on it with great pleasure. Nevertheless, he looks forward, after one and a half years of working up results and writing and correcting manuscripts, to spend more time on operating patients and sporting again.

Hopefully, after the COVID-19 pandemic, a long-cherished dream of Martijn will come true and he will perform developmental work in Africa.

Altogether, he is still convinced that he has the greatest job in the world! 


\section{List of publications}


1. Eckhardt T, Horst K, Störmann P, Bläsius F, Hofman M, Herren C, Kobbe P, Hildebrand F, Andruszkow H. Impact of chest trauma and overweight on mortality and outcome in severely injured patients. J Clin Med. 2020;9(9):E2752. Published 2020 Aug 26. doi:10.3390/jcm9092752. PMID: 32858822.

2. Teuben MPJ, Hofman M, Greven J, Shehu A, Teuber H, Pfeifer R, Pape HC, Hildebrand F. Altered cell surface receptor dynamics and circulatory occurrence of neutrophils in a small animal fracture model. [published online ahead of print, 2020 Jul 12]. Pathol Res Pract. 2020;216(10):153108. doi:10.1016/j.prp.2020.153108

3. Kobbe P, Krug P, Andruszkow H, Pishnamaz M, Hofman M, Horst K, Meyer C, Scheyerer MJ, Faymonville C, Stein G, Hildebrand F, Herren C. Early spinal injury stabilization in multiple-injured patients: Do all patients benefit? J Clin Med. 2020;9(6):1760. Published 2020 Jun 5. doi:10.3390/jcm9061760

4. Hofman M, Andruszkow H, Heyer FL, Kobbe P, Hildebrand F, Poeze M. Risk factors of non-union in intramedullary stabilized diaphyseal long bone fractures: identifying the role of fracture stabilization strategies and concomitant injuries. [published online ahead of print, 2020 Mar 5]. Eur J Trauma Emerg Surg. 2020;10.1007/s00068-020-01335-y. doi:10.1007/s00068-020-01335-y. PMID: 32140749

5. Hofman M, Kolejewska A, Greven J, Andruszkow H, Kobbe P, Tolba R, Hildebrand F, Poeze M. Gait analysis and muscle weight analysis after lower extremity fractures in a small animal model. Gait Posture. 2020;77:207-213. doi:10.1016/j.gaitpost.2020.01.022. PMID: 32058285 . PMID:32058285

6. Hofmann A, Gorbulev S, Guehring T, Schulz AP, Schupfner R, Raschke M, HuberWagner S, Rommens PM, Al Sadi O, Andruszkow H, Arand C, Biberthaler P, Danko T, Diefenbeck M, Dietz SO, Franke J, Freischmidt H, Frosch S, Gercek E, Geyer M, Glombitza M, Hanschen M, Hansen M, Henri Hessmann M, Hofman M, Holst M, Assim Kamand A, Kleber C, Kronfeld K, Marzi I, Meier S, Mu Combining Diaeresis Ller M, Peter Mu Combining Diaeresis Ller L, Nusselt T, Pfeifer E, Ruckes C, Rudig L, Sehmisch S, Stollberg-Stollberg J, Uschok S, Maximilian Vogt D, Wanner G, Wilde E, Winkelbach V, Zeitter S; CERTiFy Study Group; CERTiFy Study Group. Autologous Iliac Bone Graft Compared with Biphasic Hydroxyapatite and Calcium Sulfate Cement for the Treatment of Bone Defects in Tibial Plateau Fractures: A Prospective, Randomized, Open-Label, Multicenter Study. J Bone Joint Surg Am. 2020 Feb 5;102(3):179-193. doi: 10.2106/JBJS.19.00680. PMID:31809394

7. Teuben MPJ, Hofman M, Shehu A, Greven J, Qiao Z, Jensen KO, Hildebrand F, Pfeifer R, Pape HC. The impact of intramedullary nailing on the characteristics of the pulmonary neutrophil pool in rodents. Int Orthop. 2020 Mar;44(3):595-602. doi: 10.1007/s00264-019-04419-6. Epub 2020 Jan 3. PMID: 31900572

8. Qiao Z, Wang W, Luo P, Hofman M, Horst K, Müller-Newen G, Greven J, Hildebrand F. The Impact of Plasma-Derived Micro-vesicles From a Femoral Fracture Animal Model on Osteoblast Function. Shock. 2020 Jan;53(1):78-87. doi: 10.1097/SHK.0000000000001336. PMID: 31157718 
9. Hofman M, Rabenschlag F, Andruszkow H, Andruszkow J, Möckel D, Lammers T, Kolejewska A, Kobbe P, Greven J, Teuben MPJ, Poeze M, Hildebrand F. Effect of neurokinin-1-receptor blockage on fracture healing in rats. Sci Rep. 2019 Jul 5;9(1):9744. doi: 10.1038/s41598-019-46278-6. PMID: 31278316

10. Hofman M, Andruszkow H, Kobbe P, Poeze M, Hildebrand F. Incidence of posttraumatic pneumonia in poly-traumatized patients: identifying the role of traumatic brain injury and chest trauma. Eur J Trauma Emerg Surg. 2020 Feb;46(1):11-19. doi: 10.1007/s00068-019-01179-1. Epub 2019 Jul 3. PMID: 31270555

11. Lichte $\mathrm{P}$, Alabdulrahman H, Pishnamaz M, Hofman M, Hildebrand F, Kobbe P. Percutaneous screw techniques for the pelvic ring and acetabulum. Unfallchirurg. 2019 May;122(5):387-403. doi: 10.1007/s00113-019-0648-2. German. PMID: 31025047

12. Weber CD, Nguyen AR, Lefering R, Hofman M, Hildebrand F, Pape HC. Blunt injuries related to equestrian sports: results from an international prospective trauma database analysis. Int Orthop. 2017 Oct;41(10):2105-2112. doi: 10.1007/s00264-017-3592-1. Epub 2017 Aug 11. PMID: 28801837

13. Horst K, Garving C, Thometzki T, Lichte P, Knobe M, Dienstknecht T, Hofman M, Pape HC. Comparative study on the treatment of Rockwood type III acute acromioclavicular dislocation: Clinical results from the TightRope ${ }^{\circledR}$ technique vs. K-wire fixation. Orthop Traumatol Surg Res. 2017 Apr;103(2):171-176. doi: 10.1016/j.otsr.2016.11.009. Epub 2016 Dec 8. PMID: 27940250

14.Weber CD, Horst $\mathrm{K}$, Lefering $\mathrm{R}$, Hofman M, Dienstknecht $\mathrm{T}$, Pape HC; TraumaRegister DGU. Major trauma in winter sports: an international trauma database analysis. Eur J Trauma Emerg Surg. 2016 Dec;42(6):741-747. Epub 2015 Nov 10. PMID: 26555727

15. Sellei RM, Bauer E, Hofman M, Kobbe P, Lichte P, Garrison RL, Pape HC, Horst K. Reconstruction of a quadriceps tendon tear using Polyvinylidene fluoride sutures and patellar screw fixation: A biomechanical study. Knee. 2015 Dec;22(6):53541. doi: 10.1016/j.knee.2015.04.001. Epub 2015 May 23. PMID: 26004197

16. Hofman M, Koopmans G, Kobbe P, Poeze M, Andruszkow H, Brink PR, Pape HC. Improved fracture healing in patients with concomitant traumatic brain injury: proven or not? Mediators Inflamm. 2015;2015:204842. doi: 10.1155/2015/204842. Epub 2015 Mar 22. Review. PMID: 25873754

17. Gavenis K, Heussen N, Hofman M, Andereya S, Schneider U, Schmidt-Rohlfing B. Cell-free repair of small cartilage defects in the Goettinger minipig: the effects of BMP-7 continuously released by poly(lactic-co-glycolid acid) microspheres. J Biomater Appl. 2014 Mar;28(7):1008-15. doi: 10.1177/0885328213491440. Epub 2013 Jun 13. PMID: 23771773

18. Hofman M, Sellei R, Peralta R, Balogh Z, Wong TH, Evans JA, King K, Pape HC. Trauma systems: models of prehospital and inhospital care. Eur J Trauma Emerg Surg. 2012 Jun;38(3):253-60. doi: 10.1007/s00068-012-0192-z. Epub 2012 May 16. PMID: 26815956 
19. Hofman M, Zilkens A, Pape HC. Long-term results after polytrauma. Versicherungsmedizin. 2011 Sep 1;63(3):137-42. German. PMID: 21922716

20. Hofman M, Grommes J, Krombach GA, Schmidt-Rohlfing B. Vascular injury accompanying displaced proximal humeral fractures: two cases and a review of the literature. Emerg Med Int. 2011;2011:742870. doi: 10.1155/2011/742870. Epub 2011 May 15. PMID: 22046546

21. Schmidt-Rohlfing B, Pfeifer R, Kaneshige J, Hofman M, Knobe M, Sellei R, Pape HC. Scoring systems for outcome after knee injuries. Injury. 2011 Mar;42(3):271-5. doi: 10.1016/j.injury.2010.11.059. Epub 2011 Jan 14. Review. PMID: 21237460

22. Schmidt-Rohlfing B, Nossek M, Hofman M, Delbrück H, Hohl C. Corrective growth after vertebral body fracture in the adolescence. Z Orthop Unfall. 2009 SepOct;147(5):593-6. doi: 10.1055/s-0029-1186020. German. PMID: 19938356

23. Hofman M, Janzing HMJ, Brink PRG. Case report: "Gaat u maar even plat liggen...meneer". NTvT 2007 dec; 191-4

24.Hofman M, Janzing HMJ, Geelen PHJ, Brouwer PJ. Resultaten van botgroeistimulatie ter behandeling van 'delayed unions' van fracturen aan de bovenste extremiteit. NTvT 2007 okt; S: 143-7

25. Hofman M, Eggen PJMG. Resultaten van de 'capsular shift' volgens Rockwood. NTv0. 2006 jan; 13: 231-6

26. Hofman M, Brans BT, Van Vleuten HJ, Vandevijver N, Rahmy AIA, Heyligers IC. Case report: Een ontstoken teen; symptomatische sarcoïdose van het bot. NTvO. 2005 mrt; $12: 27-31$

\section{Book chapter}

1. Hofman M und Pape HC. (2014) 'Trauma care systems' (S. 1 - 18). In: HJ Oestern, 0 Trentz, S Uranues (Hrsg.), General Trauma Care and Related Aspects - Trauma Surgery II (1st ed.) Springer-Verlag Berlin Heidelberg 
The publication of this thesis was kindly supported by:

\section{Maastricht University}

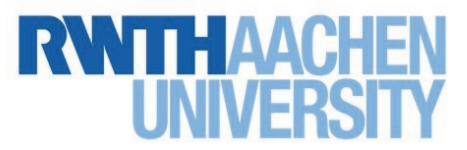

4 Maastricht UMC+

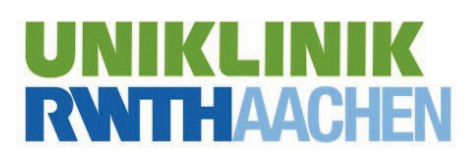

NNoldus 

